University of Tennessee Health Science Center

UTHSC Digital Commons

\title{
$5-2011$
}

\section{RNA Interference for Improving the Outcome of Islet Transplantation and Polymeric Micelle-based Nanomedicine for Cancer Therapy}

Feng Li

University of Tennessee Health Science Center

Follow this and additional works at: https://dc.uthsc.edu/dissertations

Part of the Pharmaceutics and Drug Design Commons

\section{Recommended Citation}

$\mathrm{Li}$, Feng , "RNA Interference for Improving the Outcome of Islet Transplantation and Polymeric Micelle-based Nanomedicine for Cancer Therapy" (2011). Theses and Dissertations (ETD). Paper 142. http://dx.doi.org/10.21007/etd.cghs.2011.0180. 


\title{
RNA Interference for Improving the Outcome of Islet Transplantation and Polymeric Micelle-based Nanomedicine for Cancer Therapy
}

\begin{abstract}
Ex vivo gene transfer has been used to improve the outcome of islet transplantation for treating type I diabetes. RNA interference is an effective approach for reducing gene expressions at the mRNA level. The application of RNA interference to improve the outcome of islet transplantation was reviewed in Chapter 2, where I summarized biological obstacles to islet transplantation, various types of RNAi techniques, combinatorial RNAi in islet transplantation, and different delivery strategies.

Upregulation of inducible nitric oxide synthase (iNOS) and subsequent product of radical nitric oxide (NO) impair islet $\beta$ cell function. Therefore, we hypothesized that iNOS gene silencing could prevent $\beta$ cell death. In Chapter 3 , we designed and used siRNA to silence iNOS gene in a rat $\beta$ cell line and human islets. We found that siRNA inhibited rat iNOS gene expression and NO production in rat $\beta$ cells in a dose and sequence dependent manner. iNOS gene silencing also protected these $\beta$ cells from inflammatory cytokine-induced apoptosis and increased their capacity to secret insulin. Although there was also dose and sequence dependent iNOS gene silencing and NO production in human islets, the effect of iNOS gene on apoptosis of islets was only moderate, as evidenced by $25-30 \%$ reduction in caspase 3 activity and in the percentage of apoptotic cells. Since an islet is a cluster of 20-1000 cells, the transfection efficiency of lipid/siRNA complexes into human islets was only $21-28 \%$, compared to effective transfection efficiency $(>90 \%)$ in $\beta$ cells.
\end{abstract}

Gene delivery vectors which can express a growth factor gene to promote revascularization and silence of proapoptotic genes might be good for ex vivo genetic modification of islet prior to transplantation. Thus, in Chapter 4, we constructed bipartite plasmid vectors to co-express a vascular endothelial growth factor (VEGF) CDNA and shRNA targeting iNOS gene. Firstly, shRNA sequences against human iNOS gene were screened. Then, we determined the effect of different promoters and shRNA backbones on gene silencing. The shRNA with $\mathrm{H} 1, \mathrm{U} 6$ and $\mathrm{CMV}$ promoters showed similar efficiency in iNOS gene silencing. In addition, a conventional shRNA showed better silencing of iNOS gene, compared to shRNA containing mir375 and mir30 backbones. A bipartite plasmid was also constructed with mir30-shRNA and a VEGF cDNA controlled by a single CMV promoter. This plasmid showed a better silencing effect compared with plasmid without VEGF cDNA. In conclusion, we have successfully constructed bipartite vectors co-expressing a VEGF cDNA and a shRNA against iNOS gene. These vectors could be an attractive candidate to improve the survival of transplanted islets.

The second part of research was focused on the study of polymeric micelle formulations for treating cancers. Two key elements were integrated in my research projects: development of polymeric micelle delivery systems; discovery of new therapeutics for better treatment of cancers.

The project described in Chapter 5 was carried out in collaboration with Dr. Miller's group at The University of Tennessee Health Science Center. In this study, we showed that SMART-100 effectively inhibited HepG2 cell proliferation and was able to circumvent multiple drug resistance (MDR) in cancer cells. SMART-100 inhibited P-gp activity, which may be responsible for its ability to overcome MDR. Since SMART-100 is poorly soluble in water, it was formulated in poly(ethylene glycol)-b-poly(D, L-lactide) (PEG-PLA) micelles. The solubility of SMART-100 was increased by more than $1.1 \times 10^{5}$ folds. SMART-100 loaded PEG-PLA micelles could effectively inhibit HepG2 cell growth and arrest cell cycle progression at G2/M phase, followed by cell apoptosis. Increased caspase 3 activity was also observed when HepG2 cells were treated with SMART-100. The anticancer activity of SMART-100 loaded PEG-PLA micelles was also evaluated on luciferase expressing C4-2-Luc cell lines by IVIS imaging. Our results suggest that SMART-100 has the potential to treat resistant cancers and PEG-PLA micelles can be used to formulate 
SMART-100.

We are not only interested in using commercial polymers but also interested in designing new polymers for micellar drug delivery. Therefore, the objective of study described in Chapter 6 was to design lipopolymers for hydrophobic drug delivery. In this study, poly(ethylene glycol)-block-poly(2-methyl-2-carboxyl-propylene carbonate-graft-dodecanol) (PEG-PCD) lipopolymers were synthesized and characterized by ${ }^{1} \mathrm{H}$ NMR, FTIR, GPC, and DSC. The critical micelle concentration (CMC) of PEG-PCD micelles was around $10^{-8} \mathrm{M}$ and decreased with increasing length of hydrophobic block. PEG-PCD micelles could efficiently load a model drug embelin into its hydrophobic core and significantly improve its solubility. The drug loading capacity was dependent on the polymer core structure, but the length of hydrophobic core had little effect. PEG-PCD formed both spherical and cylindrical micelles, which were dependent on the copolymer structure and composition. Lipopolymers PEG-PCD with various hydrophobic core lengths showed similar drug release profiles, which were slower than that of poly(ethylene glycol)-block-poly(2-methyl-2-benzoxycarbonyl-propylene carbonate) (PEG-PBC) micelles. Embelin loaded PEG-PCD micelles showed significant inhibition of C4-2 prostate cancer cell proliferation, while no obvious cellular toxicity was observed for blank micelles.

In Chapter 7, we studied the use of paclitaxel and lapatinib loaded lipopolymer micelles for treating MDR prostate cancers. Although paclitaxel remains effective in treating prostate cancer, its prolonged treatment develops MDR due to the over-expression of P-gp. Our hypothesis is that combination of paclitaxel and lapatinib, which is a potent $\mathrm{P}$-gp inhibitor, can overcome MDR in prostate cancers. Paclitaxel and lapatinib loaded lipopolymer micelle formulations were developed and evaluated in vitro with cell-based assay. The paclitaxel and lapatinib combination effectively inhibited in vitro MDR cancer cell proliferation, induced cell cycle perturbation and cell apoptosis. In contrast, monotherapy with paclitaxel or lapatinib alone showed minimal anticancer effect. The combination therapy was further investigated in vivo with athymic nude mice xenograft MDR tumor model. Similar to in vitro study, paclitaxel $(5 \mathrm{mg} / \mathrm{kg})$ and lapatinib $(5 \mathrm{mg} / \mathrm{Kg})$ combination therapy significantly inhibited tumor growth in vivo when compared to high dose paclitaxel $(10 \mathrm{mg} / \mathrm{kg})$ monothearpy. These studies indicate that the paclitaxel and lapatinib loaded PEG-PCD lipopolymer micelle formulation could be used to treat MDR prostate cancers.

\section{Document Type}

Dissertation

Degree Name

Doctor of Philosophy (PhD)

\section{Program}

Pharmaceutical Sciences

\section{Research Advisor}

Ram I. Mahato, Ph.D.

\section{Keywords}

RNA Interference, Islet Transplantation, Polymeric Micelles, Cancer, Multiple Drug Resistance

\section{Subject Categories}

Medicine and Health Sciences | Pharmaceutics and Drug Design | Pharmacy and Pharmaceutical Sciences 


\title{
RNA INTERFERENCE FOR IMPROVING THE OUTCOME OF ISLET TRANSPLANTATION AND POLYMERIC MICELLE-BASED NANOMEDICINE FOR CANCER THERAPY
}

\author{
A Dissertation \\ Presented for \\ The Graduate Studies Council \\ The University of Tennessee \\ Health Science Center \\ In Partial Fulfillment \\ Of the Requirements for the Degree \\ Doctor of Philosophy \\ From The University of Tennessee
}

By

Feng Li

May 2011 
Portions of Chapter 2 (C) 2011 by the Elsevier B.V. Portions of Chapter 3 (C) 2008 by the American Chemistry Society. Portions of Chapter 4 (C) 2009 by John Wiley \& Sons, Ltd. Portions of Chapter 5 (c) 2010 by the Elsevier B.V. Portions of Chapter 6 (C) 2008 by the American Chemistry Society. All other material $(2011$ by Feng Li. All rights reserved. 


\section{ACKNOWLEDGEMENTS}

I would like to take this opportunity to extend my deepest gratitude to my Advisor, Dr. Ram I. Mahato, Professor, the University of Tennessee Health Science Center, for his continuous support, patient guidance, and stimulating discussion.

I would also like to thank the other members of my committee, Dr. Ivan C. Gerling, Dr. Wei Li, Dr. Duane D. Miller, and Dr. Yongmei Wang for their guidance, comments, and assistance.

I am also grateful to my colleagues and collaborators in the University of Tennessee Health Science Center for their assistance and companionship throughout my study in Memphis. Special acknowledgments go to Dr. Yan Lu for her help and discussion in the chemical synthesis.

This dissertation is dedicated with affection to my parents Chusheng Li and Meiqin $\mathrm{Hu}$ in China, whose courage and patience have always been an inspiration to me; to Ms.

Ningsha Zhang, whose love and support has encouraged me to go through every stage of my graduate study. 


\begin{abstract}
Ex vivo gene transfer has been used to improve the outcome of islet transplantation for treating type I diabetes. RNA interference is an effective approach for reducing gene expressions at the mRNA level. The application of RNA interference to improve the outcome of islet transplantation was reviewed in Chapter 2, where I summarized biological obstacles to islet transplantation, various types of RNAi techniques, combinatorial RNAi in islet transplantation, and different delivery strategies.

Upregulation of inducible nitric oxide synthase (iNOS) and subsequent product of radical nitric oxide (NO) impair islet $\beta$ cell function. Therefore, we hypothesized that iNOS gene silencing could prevent $\beta$ cell death. In Chapter 3 , we designed and used siRNA to silence iNOS gene in a rat $\beta$ cell line and human islets. We found that siRNA inhibited rat iNOS gene expression and NO production in rat $\beta$ cells in a dose and sequence dependent manner. iNOS gene silencing also protected these $\beta$ cells from inflammatory cytokine-induced apoptosis and increased their capacity to secret insulin. Although there was also dose and sequence dependent iNOS gene silencing and NO production in human islets, the effect of iNOS gene on apoptosis of islets was only moderate, as evidenced by $25-30 \%$ reduction in caspase 3 activity and in the percentage of apoptotic cells. Since an islet is a cluster of 20-1000 cells, the transfection efficiency of lipid/siRNA complexes into human islets was only $21-28 \%$, compared to effective transfection efficiency $(>90 \%)$ in $\beta$ cells.
\end{abstract}

Gene delivery vectors which can express a growth factor gene to promote revascularization and silence of proapoptotic genes might be good for ex vivo genetic modification of islet prior to transplantation. Thus, in Chapter 4, we constructed bipartite plasmid vectors to co-express a vascular endothelial growth factor (VEGF) cDNA and shRNA targeting iNOS gene. Firstly, shRNA sequences against human iNOS gene were screened. Then, we determined the effect of different promoters and shRNA backbones on gene silencing. The shRNA with H1, U6 and CMV promoters showed similar efficiency in iNOS gene silencing. In addition, a conventional shRNA showed better silencing of iNOS gene, compared to shRNA containing mir375 and mir30 backbones. A bipartite plasmid was also constructed with mir30-shRNA and a VEGF cDNA controlled by a single CMV promoter. This plasmid showed a better silencing effect compared with plasmid without VEGF cDNA. In conclusion, we have successfully constructed bipartite vectors co-expressing a VEGF cDNA and a shRNA against iNOS gene. These vectors could be an attractive candidate to improve the survival of transplanted islets.

The second part of research was focused on the study of polymeric micelle formulations for treating cancers. Two key elements were integrated in my research projects: development of polymeric micelle delivery systems; discovery of new therapeutics for better treatment of cancers.

The project described in Chapter 5 was carried out in collaboration with Dr. Miller's group at The University of Tennessee Health Science Center. In this study, we showed 
that SMART-100 effectively inhibited HepG2 cell proliferation and was able to circumvent multiple drug resistance (MDR) in cancer cells. SMART-100 inhibited P-gp activity, which may be responsible for its ability to overcome MDR. Since SMART-100 is poorly soluble in water, it was formulated in poly(ethylene glycol)-b-poly(D, L-lactide) (PEG-PLA) micelles. The solubility of SMART-100 was increased by more than $1.1 \times 10^{5}$ folds. SMART-100 loaded PEG-PLA micelles could effectively inhibit HepG2 cell growth and arrest cell cycle progression at G2/M phase, followed by cell apoptosis. Increased caspase 3 activity was also observed when HepG2 cells were treated with SMART-100. The anticancer activity of SMART-100 loaded PEG-PLA micelles was also evaluated on luciferase expressing C4-2-Luc cell lines by IVIS imaging. Our results suggest that SMART-100 has the potential to treat resistant cancers and PEG-PLA micelles can be used to formulate SMART-100.

We are not only interested in using commercial polymers but also interested in designing new polymers for micellar drug delivery. Therefore, the objective of study described in Chapter 6 was to design lipopolymers for hydrophobic drug delivery. In this study, poly(ethylene glycol)-block-poly(2-methyl-2-carboxyl-propylene carbonate-graft-dodeca nol) (PEG-PCD) lipopolymers were synthesized and characterized by ${ }^{1} \mathrm{H}$ NMR, FTIR, GPC, and DSC. The critical micelle concentration (CMC) of PEG-PCD micelles was around $10^{-8} \mathrm{M}$ and decreased with increasing length of hydrophobic block. PEG-PCD micelles could efficiently load a model drug embelin into its hydrophobic core and significantly improve its solubility. The drug loading capacity was dependent on the polymer core structure, but the length of hydrophobic core had little effect. PEG-PCD formed both spherical and cylindrical micelles, which were dependent on the copolymer structure and composition. Lipopolymers PEG-PCD with various hydrophobic core lengths showed similar drug release profiles, which were slower than that of poly(ethylene glycol)-block-poly(2-methyl-2-benzoxycarbonyl-propylene carbonate) (PEG-PBC) micelles. Embelin loaded PEG-PCD micelles showed significant inhibition of C4-2 prostate cancer cell proliferation, while no obvious cellular toxicity was observed for blank micelles.

In Chapter 7, we studied the use of paclitaxel and lapatinib loaded lipopolymer micelles for treating MDR prostate cancers. Although paclitaxel remains effective in treating prostate cancer, its prolonged treatment develops MDR due to the over-expression of P-gp. Our hypothesis is that combination of paclitaxel and lapatinib, which is a potent P-gp inhibitor, can overcome MDR in prostate cancers. Paclitaxel and lapatinib loaded lipopolymer micelle formulations were developed and evaluated in vitro with cell-based assay. The paclitaxel and lapatinib combination effectively inhibited in vitro MDR cancer cell proliferation, induced cell cycle perturbation and cell apoptosis. In contrast, monotherapy with paclitaxel or lapatinib alone showed minimal anticancer effect. The combination therapy was further investigated in vivo with athymic nude mice xenograft MDR tumor model. Similar to in vitro study, paclitaxel $(5 \mathrm{mg} / \mathrm{kg})$ and lapatinib $(5 \mathrm{mg} / \mathrm{Kg})$ combination therapy significantly inhibited tumor growth in vivo when compared to high dose paclitaxel $(10 \mathrm{mg} / \mathrm{kg})$ monothearpy. These studies indicate that the paclitaxel and lapatinib loaded PEG-PCD lipopolymer micelle formulation could be used to treat MDR prostate cancers. 


\section{TABLE OF CONTENTS}

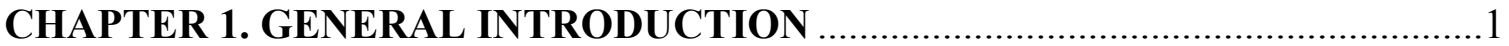

1.1. RNA Interference and Islet Transplantation ............................................ 1

1.2. Polymeric Micelle-based Nanomedicine for Prostate Cancer Therapy ........ 2

CHAPTER 2. RNA INTERFERENCE FOR IMPROVING THE

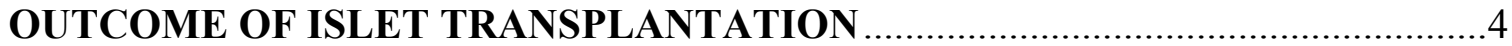

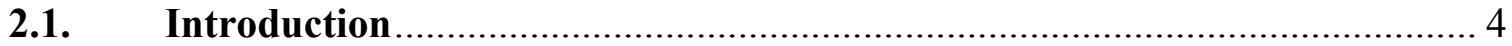

2.2. Obstacles to Islet Transplantation......................................................... 5

2.2.1. Instant blood-mediated inflammatory reaction......................................6

2.2.2. Inflammatory cytokine-induced islet cell death...................................6

2.2.3. Hypoxia and ischemic reperfusion injury ............................................10

2.2.4. Autoimmune recurrence and allogeneic immune rejection ..................10

2.3. Potential Targets for Gene Silencing .................................................... 12

2.3.1. Modulation of immune rejection .......................................................13

2.3.2. Preventing ischemic reperfusion injury and apoptosis .........................14

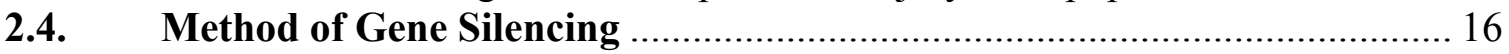

2.4.1. Types of RNAi methods .................................................................. 16

2.4.2. Design elements of siRNA and shRNA............................................17

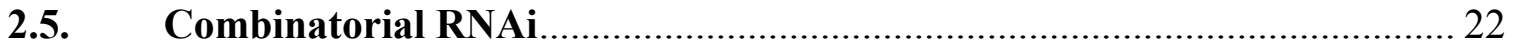

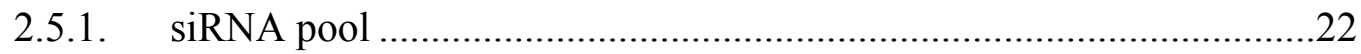

2.5.2. Co-expression of multiple shRNAs ...................................................24

2.5.3. Co-expression of shRNAs and cDNAs............................................25

2.6. Delivery Strategies for Enhanced Gene Silencing.......................................2 25

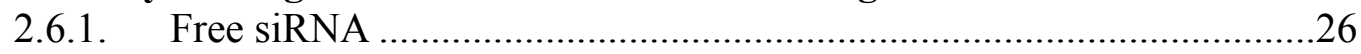

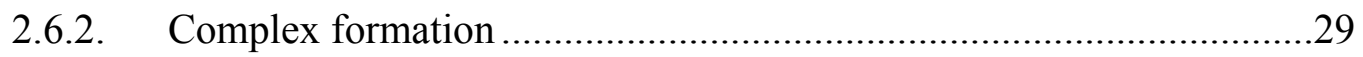

2.6.3. siRNA bioconjugation ...................................................................

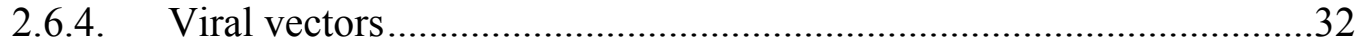

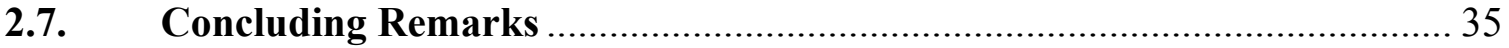

CHAPTER 3. INOS GENE SILENCING PREVENTS INFLAMMATORY

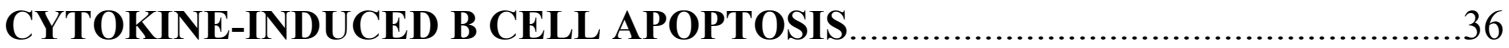

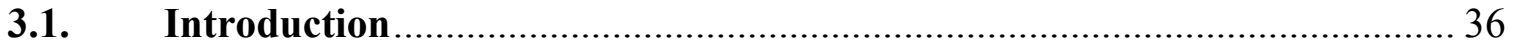

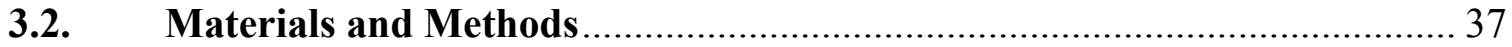

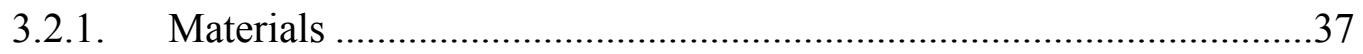

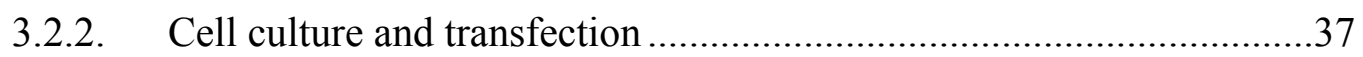

3.2.3. Fluorescence microscopy and flow cytometry ...................................40

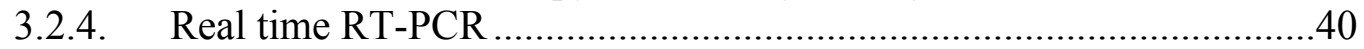

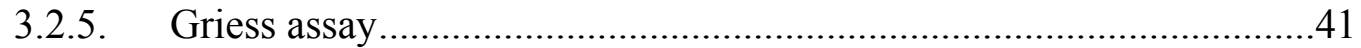

3.2.6. Effect of iNOS gene silencing on apoptotic cell death ..........................41

3.2.7. Assessment of human islet function post-transfection...........................42

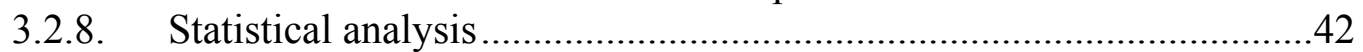

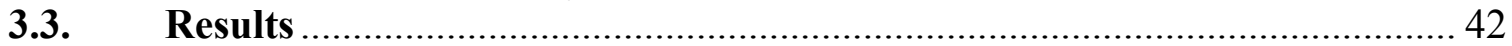

3.3.1. Transfection efficiency of lipid/siRNA complexes in rat $\beta$ cells.........42 
3.3.2. Effect of siRNA sequence on iNOS gene silencing and NO production on rat $\beta$ cells....................................................................44

3.3.3. Effect of iNOS gene silencing on rat $\beta$-cell death ...............................44

3.3.4. Transfection efficiency of lipid/siRNA complexes into human

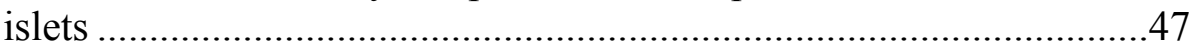

3.3.5. Effect of siRNA sequences on iNOS gene silencing in human

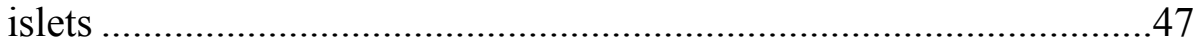

3.3.6. Effect of iNOS gene silencing on human islet cell death .....................47

3.3.7. Human islet function after iNOS gene silencing .................................50

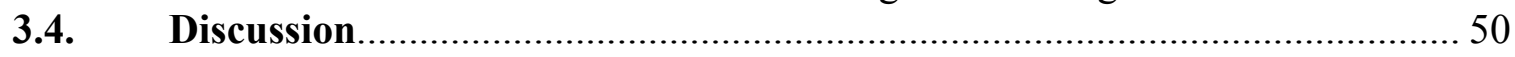

\section{CHAPTER 4. BIPARTITE VECTORS FOR CO-EXPRESSION OF A GROWTH FACTOR CDNA AND SHRNA AGAINST AN APOPTOTIC}

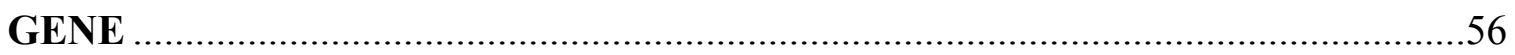

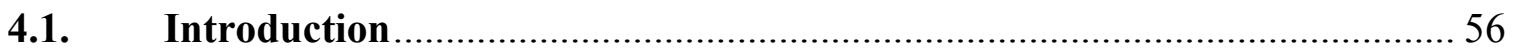

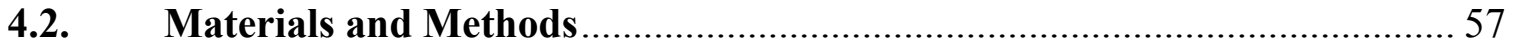

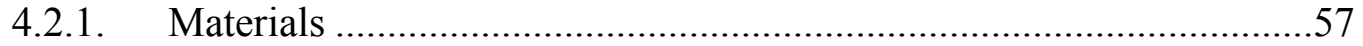

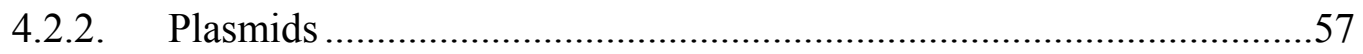

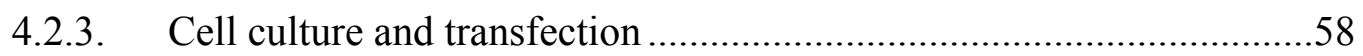

4.2.4. Real time RT-PCR .....................................................................58

4.2.5. Determination of nitric oxide production ………………………........59

4.2.6. Determination of VEGF expression from bipartite plasmids

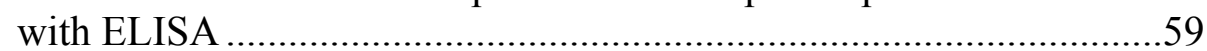

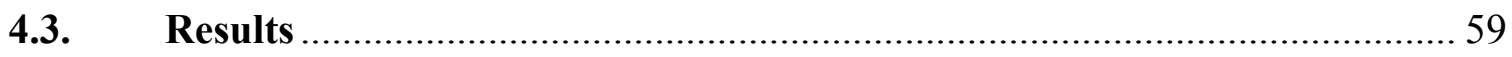

4.3.1. Effect of targeting sequence on iNOS gene silencing …….................59

4.3.2. Effect of promoters on iNOS gene silencing .......................................61

4.3.3. Effect of VEGF co-expression on iNOS gene silencing.......................61

4.3.4. Effect of mir375 and mir30 backbones on iNOS gene silencing.........61

4.3.5. Inserting VEGF gene between CMV promoter and mir30shRNA enhanced iNOS gene silencing ……………….....................65

4.3.6. Expression of VEGF from bipartite plasmids........................................65

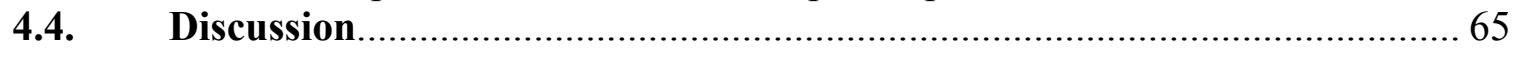

CHAPTER 5. SYNTHESIS, FORMULATION AND IN VITRO EVALUATION OF A NOVEL MICROTUBULE DESTABILIZER,

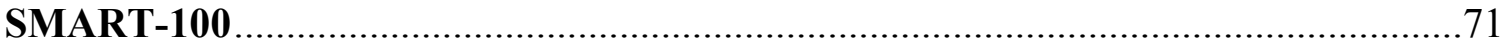

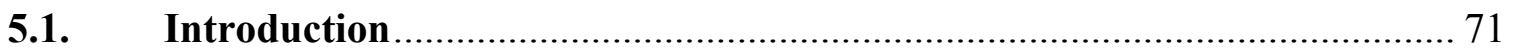

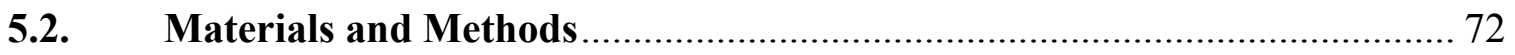

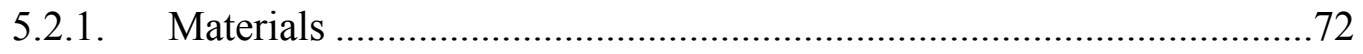

5.2.2. Synthesis of SMART-100 .............................................................72

5.2.3. Preparation and characterization micelles ...........................................73

5.2.4. Propidium iodide staining and cell cycle analysis ................................74

5.2.5. MTT assay .................................................................................

5.2.6. Calcein acetoxymethylester (calcein AM) assay ………………...........74

5.2.7. Determination of caspase 3 activity ....................................................74

5.2.8. Establishing a luciferase expression cell line for IVIS imaging...........75 


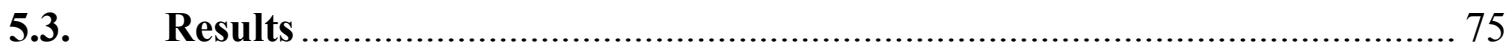

5.3.1. Synthesis and characterization of SMART-100 …………………......75

5.3.2. Anti-cancer activity of SMART-100 on HepG2 cells ...........................75

5.3.3. Anti-cancer activity of SMART-100 in resistant prostate cancer

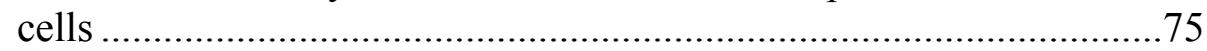

5.3.4. Micellar solubility of SMART-100 ……………..................................78

5.3.5. Anticancer effect of SMART-100 loaded PEG-PLA micelles .............78

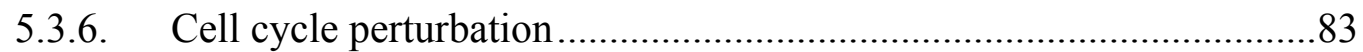

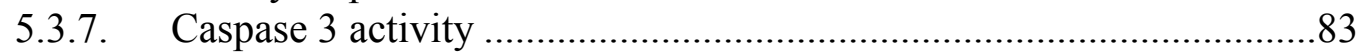

5.3.8. IVIS imaging for assessing antitumor activity....................................86

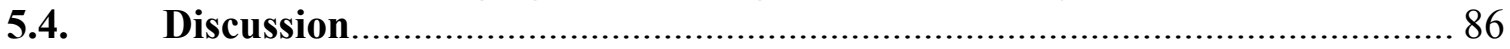

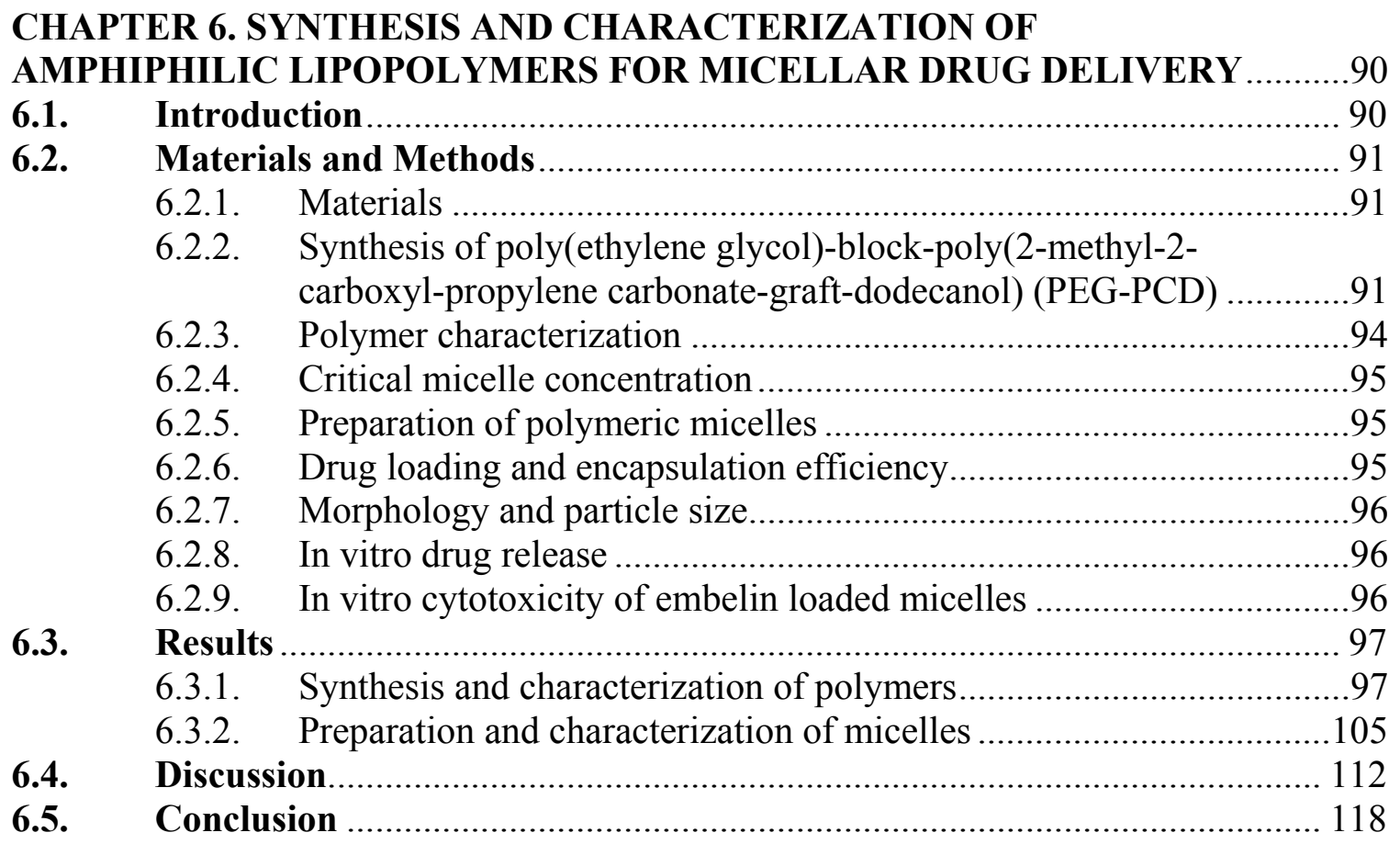

CHAPTER 7. PACLITAXEL AND LAPATINIB LOADED

LIPOPOLYMER MICELLES OVERCOME MULTIPLE DRUG

RESISTANCE IN PROSTATE CANCER

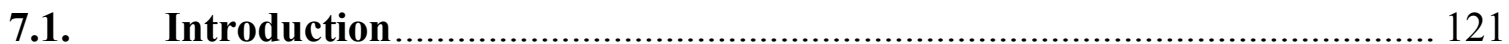

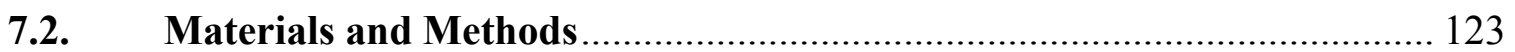

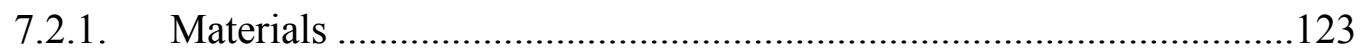

7.2.2. Synthesis of lipopolymer poly(ethylene glycol)-block-poly(2-

methyl-2-carboxyl-propylene carbonate-graft-dodecanol)

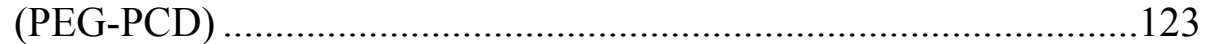

7.2.3. Preparation and characterization of lipopolymer micelles..................124

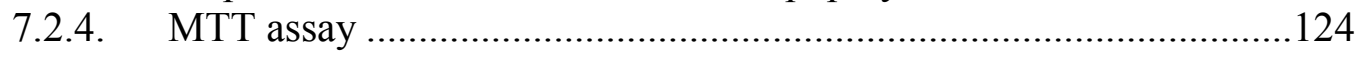

7.2.5. Calcein AM assay ......................................................................... 124

7.2.6. Propidium iodide staining and cell cycle analysis ..............................125

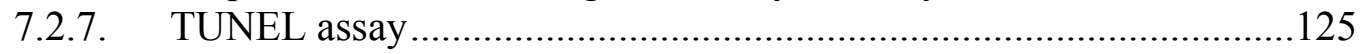


7.2.8. In vivo anticancer efficacy study ..................................................125

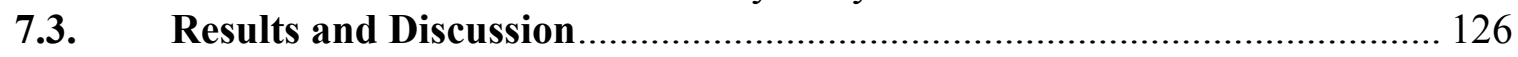

7.3.1. Effect of lapatinib and paclitaxel on MDR prostate cancer cells.......126

7.3.2. Preparation of paclitaxel and lapatinib loaded PEG-PCD lipopolymer micelle formulations ......................................................126

7.3.3. Inhibition of MDR prostate cancer cell proliferation by paclitaxel and lapatinib loaded lipopolymer micelles........................129

7.3.4. Inhibition of P-gp activity in MDR cancer cells by lapatinib............129

7.3.5. Effect of paclitaxel and lapatinib loaded micelles on cell cycle and apoptosis of MDR prostate cancer cells.

7.3.6. Lapatinib and paclitaxel loaded lipopolymer micelles overcome MDR in vivo

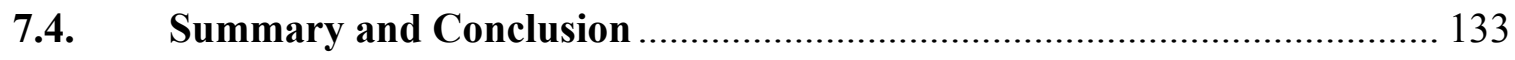

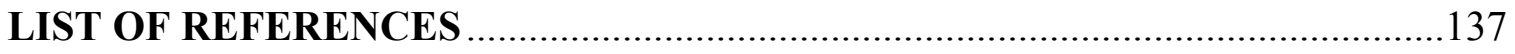

VITA 


\section{LIST OF FIGURES}

Figure 2.1 Instant blood-mediated inflammatory reaction............................... 7

Figure 2.2 Extrinsic and intrinsic pathways for islet cell apoptosis.................... 9

Figure 2.3 Autoimmune recurrence in type 1 diabetes and activation of $\mathrm{T}$ cells in host immune rejection against transplanted islets. ............... 11

Figure 2.4 Combinatorial RNAi strategies for islet genetic modification. .......... 23

Figure 2.5 Delivery strategies for gene silencing....................................... 27

Figure 2.6 Common modification introduced to siRNAs. .............................. 28

Figure 3.1 Transfection efficiency of siRNA into rat insulin producing $\beta$ cells. . 43

Figure 3.2 Effect of siRNA sequences on iNOS gene expression and

NO production in rat $\beta$ cells....................................................... 45

Figure 3.3 Effect of iNOS gene silencing on rat $\beta$ cell death............................ 46

Figure 3.4 Transfection efficiency of siRNA into intact human islets................ 48

Figure 3.5 Effect of siRNA sequence on iNOS expression on human islets. ...... 49

Figure 3.6 Effect of iNOS gene silencing on human islets cell death................. 51

Figure 3.7 Human islet function after iNOS gene silencing. ............................ 52

Figure 4.1 Effect of targeting sequence on iNOS gene silencing. ..................... 60

Figure 4.2 Effect of different promoters on iNOS gene silencing. ..................... 62

Figure 4.3 Effect of VEGF gene co-expression on iNOS gene silencing............ 63

Figure 4.4 Effect of mir375 and mir30 backbone on iNOS gene silencing......... 64

Figure 4.5 Insertion of VEGF gene between CMV promoter and mir30-shRNA enhanced iNOS gene silencing. .............................. 66

Figure 4.6 Expression of VEGF from pU6-shiNOS-CMV-VEGF and

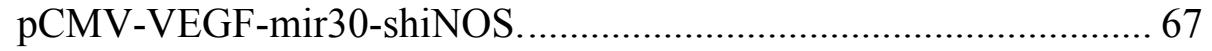

Figure 5.1 Synthesis and characterization of SMART-100............................ 76 
Figure 5.2 Anti-cancer effect of SMART-100 on HepG2 cells. ......................... 77

Figure 5.3 Anti-cancer effect of SMART-100 on MDR cancer cells. ................. 79

Figure 5.4 Effect of SMART-100 on P-gp activity....................................... 80

Figure 5.5 Solubilization of SMART-100 with PEG-PLA................................ 81

Figure 5.6 Anti-cancer effect of PEG-PLA micelles formulated SMART-100... 82

Figure 5.7 Effect of SMART-100 on cell cycle and apoptosis. .......................... 84

Figure $5.8 \quad$ Effect of SMART-100 on caspase 3 activity................................ 85

Figure 5.9 Determination of anti-cancer activity by IVIS imaging. .................. 87

Figure $6.1 \quad$ Synthesis of PEG-PCD lipopolymer............................................ 92

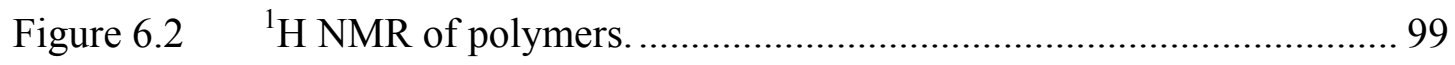

Figure $6.3 \quad$ FTIR spectra of polymers. ..................................................... 101

Figure 6.4 Gel permeation chromatography (GPC) of polymers.................... 103

Figure $6.5 \quad$ Thermal analysis of polymers by DSC . ..................................... 104

Figure 6.6 Determination of CMC values................................................ 106

Figure 6.7 Optimization of embelin loaded micelle formulations. ................... 107

Figure 6.8 Core-shell structure formed by PEG-PCD lipopolymer. .................. 108

Figure 6.9 Physical appearance and particle size of micelles........................ 110

Figure 6.10 Transmission electron microscopy (TEM) of micelles.................... 111

Figure 6.11 In vitro release of embelin from polymeric micelles..................... 113

Figure 6.12 Inhibition of C4-2 cell proliferation by PEG-PCD blank micelle and micelle formulated embelin................................................ 114

Figure 6.13 Structure of PEG-lipid and lipopolymer PEG-PCD. ...................... 116

Figure 6.14 Structure of embelin and repeating unit of hydrophobic core......... 119

Figure 7.1 Paclitaxel and lapatinib combination for treating MDR cancer........ 122 
Figure 7.2 Effect of lapatinib on reversing MDR in DU145-TXR cells.

Figure 7.3 Determine drug concentrations in paclitaxel and/or lapatinib loaded lipopolymer micelles..................................................... 128

Figure 7.4 Anticancer effect of paclitaxel and lapatinib combination on MDR DU145-TXR cells.

Figure $7.5 \quad$ Inhibition of P-gp activity by lapatinib..................................... 131

Figure 7.6 Effect of paclitaxel and lapatinib combination on cell cycle and apoptosis of MDR prostate cancers.

Figure 7.7 Detection of paclitaxel and lapatinib combination induced cell apoptosis with TUNEL staining.

Figure 7.8 In vivo anticancer effect of paclitaxel and lapatinib loaded lipopolymer micelles on xenograft MDR prostate tumors. 


\section{LIST OF ABBREVIATIONS}

$\alpha$-SMA

AAV

Adv

APCs

C3

CPPs,

DISC, e-shRNAs

FADD

FANA

GFP

HDL

HGF

HLA

IFN- $\gamma$

IFN $\gamma \mathrm{R} 1$

IKK

IL-1AcP

IL-1ß

iNOS

IRAK

ITN

JAK $1 / 2$

JDRF

JNK

LCMV

LDL

IhRNA

LNA

MCP-1

MDA-5

$\mathrm{MHC}$

MLR

MOE

MyD88

NCRR

$\mathrm{NIH}$

NO

PEC micelles

PEG

PEG-PBC $\alpha$-smooth muscle actin

recombinant adeno-associated virus

adenoviral

antigen presenting cells

complement 3

cell-penetrating peptides

death-inducing signaling complex

extended hairpin RNAs

fas-associated death domain

2 '-O-fluoro- $\beta$-D-arabinonucleotide

green fluorescent protein

high density lipoprotein

hepatocyte growth factor

human leukocyte antigen

interferon- $\gamma$

IFN $\gamma$ receptor 1

I $\mathrm{B}$ kinase

IL-1 receptor accessory protein

interleukin-1ß

inducible nitric oxide synthase

IL-1R1 activated kinase

immune tolerance network

janus tyrosine kinase 1 and 2

juvenile diabetes research foundation

c-jun N-terminal kinase

lymphocytic choriomeningitis virus

low density lipoprotein

long hairpin RNA

locked nucleic acid

monocyte chemoattractive protein-1

melanoma differentiation-associated gene-5

major histocompatibility complex

mixed lymphocyte reaction

2'-O-(2-methoxyethyl)

myeloid differentiation factor 88

national center for research resources

national institutes of health

nitric oxide

polyelectrolyte complex micelles

poly (ethylene glycol)

poly(ethylene glycol)-block-poly(2-methyl-2-

benzoxycarbonyl-propylene carbonate) 
PEG-PCC

PEG-PCD

PEI

PKR

PLL

RFP

RIG-1

RISC

ROS

shRNA

SOCS

STAT1

tet $\mathrm{O}$

TLR

TNF- $\alpha$

Tol-DC

TRADD

TRAF2

TRAF6

UTR

VEGF

VSV-G

2'-F

2'OMe poly(ethylene glycol)-block-poly(2-methyl-2-carboxylpropylene carbonate)

poly(ethylene glycol)-block-poly(2-methyl-2-carboxylpropylene carbonate-graft-dodecanol)

polyethylenimine dsRNA-dependent protein kinase $\mathrm{R}$

poly (L-lysine)

red fluorescent protein

retinoic acid-inducible gene-1

RNA-induced silencing complex

reactive oxygen species

short hairpin RNA

suppressor of cytokine signaling

signal transducers and activators of transcription 1

tetracycline operator

toll-like receptor

tumor necrosis factor- $\alpha$

tolerogenic DC

TNF- $\alpha$ associated death domain

TNF-receptor-associated factor-2

TNF-receptor-associated factor- 6

untranslated region

vascular endothelial growth factor

vesicular stomatitis virus glycoprotein

2'-fluoro

2'-O-methyl 


\section{CHAPTER 1. GENERAL INTRODUCTION}

\subsection{RNA Interference and Islet Transplantation}

Islet transplantation is a potential cure for type 1 diabetes mellitus. Despite recent therapeutic success, it is still not common because a large number of transplanted islets are damaged by multiple challenges: including instant blood mediated inflammatory reaction, hypoxia/reperfusion injury, inflammatory cytokines, and immune rejections. As a potent approach to selectively degrade target mRNA, RNA interference (RNAi) was developed rapidly as a novel therapeutic strategy. The use of RNAi technologies to down-regulate the expression of harmful genes has the potential to improve the outcome of islet transplantation.

In the first part of this dissertation, I reviewed how to use RNAi technique to improve the outcome of islet transplantation. And the following specific topics were discussed:

- Develop potential RNAi targets based on biological obstacles for islet transplantation.

- Design effective RNAi strategies: chemically synthesized small interfering RNA (siRNA), vector-based short hairpin RNA (shRNA), and their critical design elements (such as sequences, promoters, backbones).

- Use combinatorial RNAi in islet transplantation.

- Enhance gene silencing with various delivery strategies: chemical modification of siRNA, complex formation, bioconjugation, and viral vectors.

Inducible nitric oxide synthase (iNOS) are up-regulated after islet transplantation, which will increase the production of radical nitric oxide (NO), and impair islet $\beta$ cell viability and function. This is one of the major reasons lead to the failure of islet transplantation. In the first project, we hypothesized that iNOS gene silencing will prevent $\beta$ cell death and improve the survival and function of islets. Following specific aims were studied in this project:

- Optimize the siRNA sequence, dose, and transfection conditions to effectively silence iNOS gene expression in a rat $\beta$ cell line and human islets.

- Determine the effect of iNOS gene silencing on NO production, inflammatory cytokine-induced cell apoptosis, and insulin production.

It is also known that multiple reasons are responsible for the damage of transplanted islets, including inflammation and cell apoptosis, poor revascularization and hypoxia. In the following project, we are interested in developing bipartite vectors for co-expression of a growth factor cDNA, which can promote the revascularization of islets, and shRNA against an apoptotic gene to improve the viability of islets. Therefore, we have the following specific aims:

- Screen shRNA sequences for silencing human iNOS.

- Study the effect of different promoters (such as H1, U6 and CMV) and miRNA 
backbone (mir375 and mir30) on gene silencing.

- Develop bipartite plasmid vectors co-expressing mir30-based shRNA and VEGF cDNA with a single CMV promoter.

- Develop bipartite plasmid vectors co-expressing shRNA and cDNA under separated expression cassettes.

\subsection{Polymeric Micelle-based Nanomedicine for Prostate Cancer Therapy}

Polymeric micelles can solubilize hydrophobic drugs and effectively deliver drugs into tumors by the enhanced permeability and retention (EPR) effect. Several polymeric micelle formulations are currently under clinical trials or approved for treating cancers [1, 2]. For an example, PEG-PLA micelles (Genexol-PM) have been successfully used as a Cremophor EL-free formulation for paclitaxel. It was approved and marketed at Korean in February 2007 and currently under clinical trial in USA (http://www.clinicaltrials.gov). In this section, our research was focused on the development of polymeric micelle-based nanomedicines for treating cancers.

The first project in polymeric micelles formulation development was carried out in collaboration with Dr. Miller's research group. They have discovered a potent anticancer compound, substituted methoxybenzoyl-ary-thiazole-100 (SMART-100). SMART-100 showed excellent anticancer activity in many cancer cells and even in MDR prostate cancers. However, the application of SMART-100 for chemotherapy was limited by its low aqueous solubility (less than $2 \mathrm{ng} / \mathrm{mL}$ ). We are interested in developing a polymeric micelle formulation for SMART-100, and have the following specific aims:

- Determine in vitro anticancer effect of SMART-100 on HepG2 Cells and MDR prostate cancer cells (DU145-TXR).

- Prepare and characterize SMART-100 loaded PEG-PLA micelle formulations.

- Determine drug solubility and loading efficiency of SMART-100 in polymeric micelles.

- Determine in vitro anticancer effect of SMART-100 loaded PEG-PLA micelles: cell cycle perturbation and apoptosis, caspase 3 activity assay, IVIS imaging.

In the following study, we designed and synthesized a lipopolymer for micellular delivery of embelin, which is an XIAP inhibitor and showed good anticancer activity on prostate cells in combination with bicalutamide. We have following specific aims:

- Design and optimize the synthesis procedure of lipopolymers poly(ethylene glycol)block-poly(2-methyl-2-carboxyl-propylene carbonate-graft-dodecanol).

- Characterize lipopolymers with ${ }^{1} \mathrm{H}$ NMR, FTIR, GPC, and DSC.

- Determine the effect of hydrophobic core length and structure on the CMC value of polymers.

- Determine the effect of core structure and length on drug loading, micelle morphology, and in vitro drug release. 
- Evaluate the anticancer effect of embelin loaded lipopolymer micelle in vitro with prostate cancer cells.

In the last project, we developed paclitaxel and lapatinib combination therapy to overcome MDR in prostate cancers. Lipopolymer PEG-PCD was used to prepare paclitaxel and lapatinib loaded micelles. Thus, the following specific aims were tested in this project:

- Synthesize and characterize PEG-PCD lipopolymers.

- Develop a HPLC method to simultaneously determine paclitaxel and lapatinib in lipopolymer micelles.

- Optimized and characterize paclitaxel and lapatinib loaded lipopolymer PEG-PCD micelle.

- Determine the effect of paclitaxel and lapatinib combination on MDR prostate cancer cells in vitro: MTT assay, TUNEL assay, cell cycle analysis.

- Determine the effect of paclitaxel and lapatinib combination in vivo with athymic nude mice xenograft MDR tumor model. 


\section{CHAPTER 2. RNA INTERFERENCE FOR IMPROVING THE OUTCOME OF ISLET TRANSPLANTATION*}

\subsection{Introduction}

The first attempt to transplant pancreatic tissue was performed 29 years before the clinical introduction of insulin, when a young boy dying of diabetic ketoacidosis had three small pieces of sheep's pancreas implanted beneath his skin [3]. But the patient died after 3 days. In 1966, the first pancreatic allotransplantation was performed at the University of Minnesota [4]. Since then, more than 30,000 pancreas transplantations were carried out worldwide. In 2008, approximately 1300 patients were transplanted with pancreas in the United States with the majority of them being simultaneous pancreaskidney transplantations [5]. Although there are some beneficial effects, pancreas transplantation remains an invasive procedure with significant mortality and morbidity [6]. Pancreas transplantation is also limited by the death of organs, and the need for nonspecific immune suppression.

Insulin-producing $\beta$ cells in the islets of Langerhans are the target of autoimmune aggression in type I diabetes, and non- $\beta$ cells and the exocrine pancreas is unaffected. The islets constitute only a tiny fraction (1\%) of the whole pancreas and it is functionally unnecessary to transplant the whole pancreas when only its endocrine tissue is required. Islet transplantation provides a less invasive alternative approach for the treatment of type 1 diabetes with reduced antigen load, relative simplicity, and low morbidity. However, since the initiation of clinical islet transplantation from early 1970s, most of these trials were failed, until the breakthrough at the University of Alberta in Edmonton, Canada. The "Edmonton protocol" was published in 2000, reporting that seven patients with type I diabetes became insulin independent after receiving islet transplantation with a prednisone-free protocol [7].

The Edmonton protocol has been replicated and further modified worldwide with many successes. In 2001, the National Center for Research Resources (NCRR) of the National Institutes of Health (NIH) established ten islet resource centers with the mission of (1) providing high quality human islets for both clinical and basic research use; (2) optimizing processes for islet isolation, purification, and storage; and (3) developing the methods for characterization of islets (http://icr.coh.org). In the same year, the Immune Tolerance Network (ITN) with the joint help from the Juvenile Diabetes Research Foundation (JDRF) and NIH began a trial at nine centers worldwide to perform transplants in 40 subjects to determine if the Edmonton results could be reproduced on a broad scale. The ITN aims at developing a core group of excellent clinical islet transplant centers for future testing of tolerance-based trials (http://www.immunetolerance.org). From 1999 to 2008, around 400 patients received allogeneic islet transplantation [8,9].

* Adapted with permission. F. Li, R.I. Mahato, RNA interference for improving the outcome of islet transplantation, Adv Drug Deliv Rev, 63 (2011) 47-68. 
Despite these successes, only less than $10 \%$ of the recipients remain insulin independent for up to 5 years, and most recipients return to insulin because the islet function decreased over time [10]. After transplantation, Islet grafts face multiple challenges including innate and adaptive immune rejection, toxicity associated with immune suppressive agents, and insufficient islet revascularization [11, 12]. Therefore, islets isolated from two to four donors are needed for single patient islet transplantation [13]. At present, even with expansion in the currently available organ donor pool, islet transplantation will only benefit about $0.5 \%$ of potential recipients [14].

Ex vivo genetic modification of islets before transplantation has the potential to overcome several problems associated with islet transplantation [12, 13, 15-19]. Growth factor gene expression has the potential to promote islet revascularization; while antiapoptotic gene expression can reduce inflammation and immune rejection; and prevent islet cell apoptosis. This strategy can help to reduce the number of islets needed for each recipient and prolong the time that a recipient can maintain insulin independence after transplantation. The genetic modification of islets could be the over-expression of protective genes or inhibition of harmful gene expression [20]. Antisense oligonucleotides (ODNs), ribozymes, DNAzymes, and RNA interference (RNAi) are major approaches which are currently being used to sequence specifically reduce or inhibit gene expression. Among these approaches, RNA interference (RNAi) is relatively new and evolutionally conserved biologic process that regulates gene expression using small interfering RNA (siRNA) mediated sequence specific, post-transcriptional gene silencing [21, 22]. Since the discovery of RNAi by Fire and Milo in 1998, it has been widely used as a tool for basic research as well as a potential therapeutics [23-26]. The application of RNAi and other approaches have been extensively reviewed [27]. siRNA molecules can recycle between different copies of mRNA, while antisense ODN can only block the translation of one mRNA before its degradation [28]. Therefore, siRNA is more effective than antisense ODN in reducing target gene expression [29]. Miyagishi et al. has demonstrated that the $\mathrm{IC}_{50}$ for siRNA was about 100 -fold lower that of the antisense [30]. Similarly, Bertrand et al. has reported that siRNAs are more efficient in silencing target gene and its effect is lasting longer. In addition, RNAi could also be achieved though the delivery of shRNA, which is expressed endogenously and has longer gene silencing effect [29].

In this review, we will discuss the biological obstacles to islet transplantation and potential RNAi targets; principles and design element for RNAi; and delivery approaches.

\subsection{Obstacles to Islet Transplantation}

Since the success of Edmonton protocol, significant progress has been made in human islet transplantation. However, this protocol is still not widely used mainly due to the fact that islets from several donors are needed for a single recipient, since majority of the islets are damaged prior and post transplantation. The destruction of transplanted islets is

a complex problem with many facets including enzyme and mechanical damages induced 
by the isolation process, hypoxia, inflammatory reaction, and immune rejection. Since many of these factors are inter-related, a thorough understanding of these molecular processes will help us to develop more effective therapeutic interventions.

\subsubsection{Instant blood-mediated inflammatory reaction}

Due to non-immunological and innate immunological factors, 50\%-70\% islets lose their viability immediately after transplantation by instant blood-mediated inflammatory reaction [31-33]. This phenomenon is also called "early graft loss" or "primary nonfunction." The pathophysiology of blood-mediated inflammatory reaction involves platelet binding and activation, coagulation, complement activation, and infiltration of neutrophils, monocytes, and macrophages (Figure 2.1) [31]. Activation of coagulation could be triggered through both the intrinsic pathway by islet surface collagen residues and the extrinsic pathway by tissue factors or monocyte chemoattractive protein-1 (MCP1) $[34,35]$. Complement is possibly activated through the alternative pathway $[36,37]$. Infiltration of these immune cells could be observed within $1 \mathrm{~h}$ after coagulation and complement activation [38]. There are multiple interactions between coagulation, complement, and inflammatory cells, which are responsible for the infiltration of inflammatory cells. Neutrophils and monocytes interact with activated platelets through P-selectin over-expressed on platelets [39]. Macrophages could directly be activated by tissue factor, fibrin, or fibrinogen [40]. Soluble C3a and C5a produced through complement activation are potent chemoattractants for neutrophils and macrophages [36]. The effects of blood-mediated inflammatory reaction are multiple. Firstly, both monocytes and macrophages are major phagocytes leading to the destruction of islet cells. Secondly, proinflammatory cytokines lead to islet cell apoptosis and necrosis. Last but not the least, the infiltrating neutrophils and macrophages will eventually induce subsequent adaptive immune response [41].

\subsubsection{Inflammatory cytokine-induced islet cell death}

Mechanical and enzymatic stresses during the islet isolation, purification and transfection process result in the activation of resident islet macrophages, which release several proinflammatory cytokines including interleukin-1ß (IL-1ß), tumor necrosis factor- $\alpha$ (TNF- $\alpha$ ), and interferon- $\gamma$ (IFN- $\gamma)$. In addition, infiltrating host immune cells (monocytes, neutrophils) also produce inflammatory cytokines [42]. These proinflammatory cytokines are responsible for the non-specific inflammation and the damage to transplanted islets during the early stage of transplantation. The exact source and timing of proinflammatory cytokine production still remains elusive and greatly depends on the islet isolation, purification and transplantation protocols. For an example, Kupffer cells produce inflammatory cytokines, NO, and reactive oxygen species (ROS), which are toxic to intraportally transplanted Islets [43-45]. The release of cytokines by islet resident macrophages is known to occur during in vitro culture prior to transplantation [46]. The levels of IL-1 $\beta$ and TNF- $\alpha$ increase soon after transplantation, and maximum level was reached at $3 \mathrm{~h}$ for IL-1 $\beta$ and at $6 \mathrm{~h}$ for TNF- $\alpha$. Depletion of kupffer cells significantly 


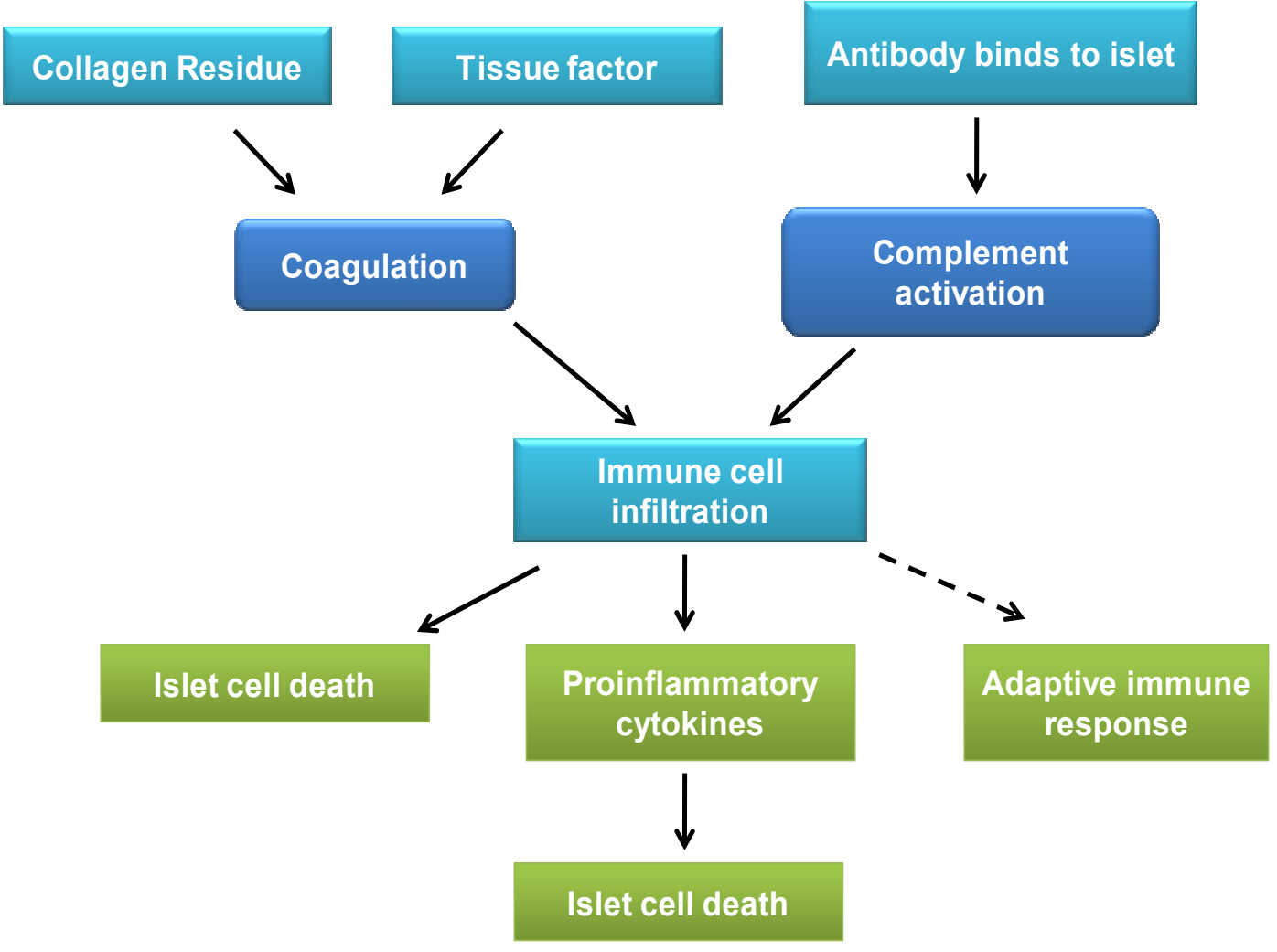

Figure 2.1 Instant blood-mediated inflammatory reaction. 
reduced the levels of IL- $1 \beta$ and TNF- $\alpha$, indicating the role of macrophage in producing inflammatory cytokines [47]. Another study also showed that IL-1 $\beta$ mRNA was detectable in islet immediately after isolation and was increased after transplantation. IL$1 \beta$ mRNA increased by 9 fold on day 1 , by 7 fold on day 3 after transplantation, and decreased to baseline level on day 7 [48]. It has also been reported that the expression of proinflammatory cytokines (including IL-1 $\beta$ and TNF- $\alpha$ ) were observed at $8 \mathrm{~h}$ after transplantation and declined after $24 \mathrm{~h}$. In the same study, however, IFN- $\gamma$ was not observed until $48 \mathrm{~h}$ post transplantation [49]. Ozasa et al. reported that IFN- $\gamma$ mRNA was observed in allogeneic transplantation at 1,3,5, and 7 days after transplantation with a peak at day 5, however, it was almost absent in syngeneic transplantation [50]. Although the exact source and level of proinflammatory cytokines production varies among different transplantation protocols used, some common intracellular signal pathways are activated. These cytokines activate JNK/p38, NF- $\kappa \beta$, STAT-1, up-regulate the expression of several significant genes, and finally result in $\beta$ cell death (Figure 2.2) [36-37].

The binding of IL- $1 \beta$ to its receptor IL-1R1 leads to the docking of the IL-1 receptor accessory protein (IL-1AcP) and followed by the recruitment of IL-1R1 activated kinase (IRAK) through an adaptor protein named myeloid differentiation factor 88 (MyD88). Recruitment of IRAK leads to the activation of TNF-receptor-associated factor-6 (TRAF6), resulting in the phosphorylation and degradation of I $\kappa \mathrm{B}$. NF- $\kappa \mathrm{B}$ is then released from inhibitory $\mathrm{I} \kappa \mathrm{B}$, translocates into the nucleus, and induces the expression of multiple genes, including IL-1, IL-6, TNF- $\alpha$, and iNOS [51, 52].

IFN- $\gamma$ binds to IFN $\gamma$ receptor 1 (IFN $\gamma \mathrm{R} 1$ ), which recruits IFN $\gamma$ receptor 2 (IFN $\gamma \mathrm{R} 2$ ). IFN $\gamma \mathrm{R} 1$ and IFN $\gamma \mathrm{R} 2$ are associated with Janus tyrosine kinase 1 and $2(\mathrm{JAK} 1 / 2)$ and results in the activation of JAK1 and JAK2. Then signal transducers and activators of transcription 1 (STAT1) is activated by JAK2 and then translocates to the nucleus, where it binds to the regulatory regions of different genes [53]. In addition, the ischemiainduced STAT1 also regulates caspase gene expression through the activation of transcription factor IRF-1 [54].

Upon binding of TNF- $\alpha$, TNF receptors form trimers and undergo conformational change, leading to the exposure of intracellular death domain. The death domain interacts with TNF- $\alpha$ associated death domain (TRADD), which serves as an adaptor to initiate three signal pathways, including the activation of NF- $\kappa \mathrm{B}$, activation of MAPK pathway, and induction of apoptosis [55-57]. TNF receptor-associated factor 2 (TRAF2) and receptor-interacting protein (RIP) are associated with TRADD to recruit and activate kinase IKK, which in turn releases NF- $\kappa$ B by degradation of I $\kappa \mathrm{B}$ [55]. TRAF2 also activates MAPK pathways and leads to the protein kinases such as c-jun $\mathrm{N}$-terminal kinase (JNK) and p38 [58]. Apoptosis are activated through both MAPK pathway (direct activation of caspase 3 ) or FADD mediated activation of caspase 8 , which in turn leads to the activation of effector caspase, such as caspase 3 [57].

Under in vitro conditions, IL-1 $\beta$ induces functional impairment in the mouse and rat $\beta$ cells, and prolonged exposure (6-9 days) to IL- $1 \beta$ in combination with IFN- $\gamma$ and TNF- $\alpha$ leads to human, mouse, and rat $\beta$ cell death [52]. The fate of $\beta$ cells after exposure to 


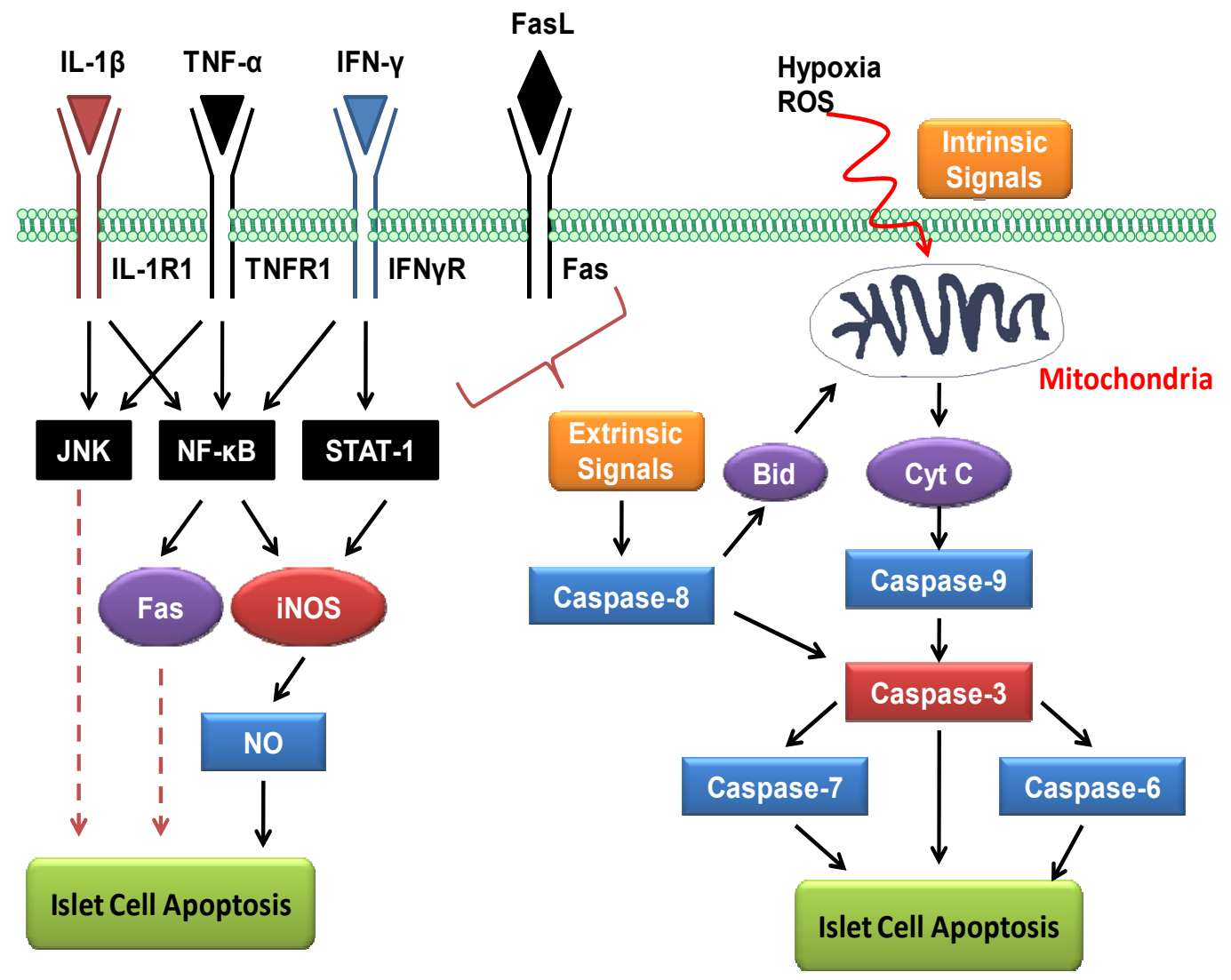

Figure 2.2 Extrinsic and intrinsic pathways for islet cell apoptosis. 
inflammatory cytokines depends on the type of cytokines, time course and severity of perturbation to the key $\beta$ cell gene networks [52,59]. Inflammatory cytokines upregulate or downregulate around 200 genes, which are either protective or deleterious to $\beta$ cell survival and function $[59,60]$. These genes are related to metabolism, signal transduction, and transcription factors, suggesting that $\beta$ cells are making attempt to adapt to the effect of cytokine exposure [59]. Several of these genes are putative targets of transcription factor NF- $\kappa B$ [61]. Transcriptional activity of NF- $\kappa B$ is related to the expression of proapoptotic genes in $\beta$ cells [62]. The activation of NF- $\mathrm{kB}$ is a necessary step for inducible nitric oxide synthase (iNOS) expression and nitric oxide (NO) production [61]. The cytotoxic effect of cytokines is mediated through the production of radical $\mathrm{NO}$ as well as other free radicals, such as peroxynitrite and superoxide [48, 63]. Increased iNOS gene expression leads to an early inflammatory process in islet transplantation, suggesting iNOS is an important mediator of graft inflammation and islet damage in the early stage of islet transplantation. Inflammatory cytokines initiate $\beta$ cell apoptosis mainly though the extrinsic pathway and function through several molecules in the death receptor pathway, such as Fas (CD95), Fas-associated death domain (FADD), and the death-inducing signaling complex (DISC), leading to the activation and release of caspase 8 , which in turn activate caspase 3 [64-66].

\subsubsection{Hypoxia and ischemic reperfusion injury}

In an intact pancreas more than $15 \%$ of the total blood flow goes to islets, which accounts for only $1 \%$ of the pancreatic tissue [67]. During isolation and purification process, the capillary network for blood supply is destroyed; therefore, oxygen and nutrients are solely supplied through diffusion. The oxygen tension in portal vein (where the islets are transplanted) is $8-10 \mathrm{mmHg}$, which is much lower than that in the intact pancreas $\left(\mathrm{PO}_{2}=\right.$ $40 \mathrm{mmHg}$ ) [68, 69]. Although the angiogenesis initiates soon after transplantation, revascularization of islets may take up to 10-24 days [70]. Therefore, newly transplanted islets are under persistent hypoxic conditions prior to and post transplantation, until revascularization process is completed. The reperfusion following prolonged ischemia causes injury, commonly known as ischemia/reperfusion (IR) injury. For an example, IR injury in the liver activates Kupffer cells, which will produce inflammatory cytokines, ROS and NO, resulting in islet cell apoptosis [71]. Hypoxia induces the intrinsic pathway of apoptosis though the disruption of the mitochondrial integrity, induction of cytochrome $\mathrm{C}$ release, and the activation of caspase 9 and 3 [64-66]. Nutrient deprivation can also induce islet apoptosis through the intrinsic pathway [64].

\subsubsection{Autoimmune recurrence and allogeneic immune rejection}

In addition to hyperacute rejection, acute rejection, and chronic rejection experienced during the transplantation of other organs such as heart, lung, liver, and kidney, transplanted islets are under additional attacks due to autoimmune rejection against $\beta$ cells in type I diabetic patients. In the following paragraph, we will briefly summarize the immunology of islet transplantation (Figure 2.3). 


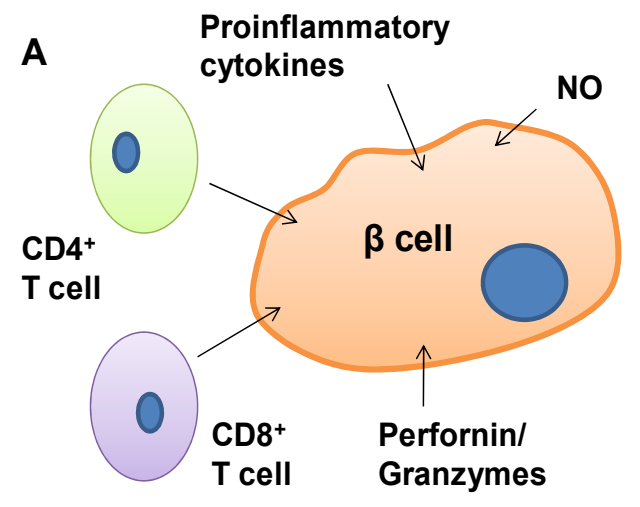

Autoimmune recurrence in T1DM

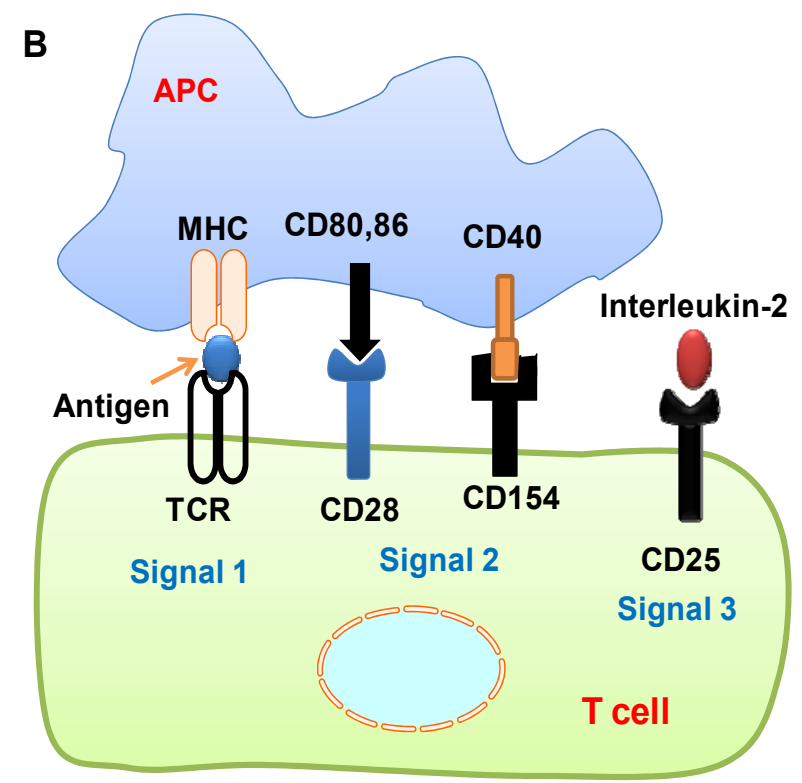

Activation of T-cells in host immune response to transplanted islets

Figure 2.3 Autoimmune recurrence in type 1 diabetes and activation of $T$ cells in host immune rejection against transplanted islets. 


\subsubsection{Autoimmune recurrence}

Type I diabetes is caused by autoimmune mediated damage of islet $\beta$ cells. It is characterized by the presence of $\beta$ cell reactive $T$ cells, proinflammatory cytokines, and other molecules. Transplanted islets are also attacked by the same stresses that destroy host $\beta$ cells. Among them, CD8 $+\mathrm{T}$ cells are one of the major players in destroying $\beta$ cells. The infiltration of CD8+ T cells is observed in the pancreas of type 1 diabetic patients as well as transplanted pancreas $[72,73]$. In addition, activated CD4+ T cells are also involved in the killing of $\beta$ cells. Reduced insulitis is observed in the absence of CD4+ $\mathrm{T}$ cells [74]. The activation of CD8+ $\mathrm{T}$ cells is dependent on CD4+ T cells. CD8+ $\mathrm{T}$ cells kill target cells through direct contact and mediated by perforin and granzyme $\mathrm{B}$. Perforin can disturb target cell membrane integrity and facilitate the release of granzyme $\mathrm{B}$ into the cytoplasm of target cells, whereas granzyme B cleaves proapoptotic molecule Bid. Activated Bid sequesters anti-apoptotic Bcl-2 molecules and triggers $\beta$ cell apoptosis through the intrinsic apoptotic pathway [75-77]. Fas is also upregulated in pancreatic $\beta$ cells of type 1 diabetic patients and causes $\beta$ cell apoptosis through FasL-Fas interaction $[78,79]$.

\subsubsection{Allogeneic immune rejection}

Successful transplantation of allogeneic islets is difficult, because transplanted islets are recognized not only by the host immune system, but the adaptive immune rejections are also activated [80]. Failure of allogeneic islet transplantation is correlated with increased $\mathrm{T}$ cell reactivity or alloantibody titers $[81,82]$. Antigen presenting cells (APCs) process and present peptide fragments of various surface, secreted and shed protein from the donor. APCs from both donor and host (including dendritic cells, macrophages, passenger leukocytes, and mononuclear cells) are involved in the antigen presentation. After maturation, APCs provide three signals to fully activate T cells: signal 1, recognition of major histocompatibility complex (MHC) and the bound peptide by the $\mathrm{T}$ cell receptor on the host T cells; signal 2, interaction of co-stimulation signals (such as CD40, CD80/86) with their corresponding receptors on host T cells; signal 3, soluble cytokines (IL-12, IL-10, IL-2) further stimulate the proliferation and differentiation of T cells $[83,84]$. All of these three signals are required; otherwise, it will result in the apoptosis, anergy, and differentiation of $\mathrm{T}$ cells. In addition, anti-donor antibodies are also developed in most allograft recipients after immunosuppressive medication is discontinued. The presence of alloantibodies usually lead to the graft failure of islets from donors with the recognized human leukocyte antigen (HLA) allodeterminants [81].

\subsection{Potential Targets for Gene Silencing}

As a potent approach for inhibiting aberrant protein expression, RNAi can theoretically be used to silence any gene involved in the biological processes that lead to the damage of islets. However, due to the complexity of pathophysiology of islet transplantation, only some key mediators are potential targets for RNAi-based intervention. A wide variety of 
potential therapeutic targets are investigated in the past, which are discussed in the following paragraphs.

\subsubsection{Modulation of immune rejection}

Immunosuppressive agents mediated non-specific immunosuppression is commonly used to reduce the immune rejection in islet transplantation, however, even the most isletfriendly immunosuppressive regimen are toxic to islets. For example, the treatment of islets with sirolimus (also known as rapamycin) or tacrolimus (also known as FK-506 or Fujimycin) causes islet apoptosis [85]. Immune modulation with other biological approaches could also provide alternative ways to reduce immune rejection [12].

\subsubsection{Genetic modification of dendritic cells}

Dendritic cells are one of the most important APCs, which play a critical role in the regulation of immune responses. The activation and maturation of $T$ cells relies on the signals from APCs. T cells will undergo apoptosis, if any one of these signals is blocked. DCs are genetically modified to act as a suppressor of immune responses. These DCs are called tolerogenic DC (Tol-DC). Clinically, we could isolate DCs from the host and then administer them back to the host after making them tolerogenic. Inhibition of the genes associated with DC maturation is one of the promising approaches to generate Tol-DC. $\mathrm{NF}-\kappa \mathrm{B}$ is an essential transcription factor for DC differentiation and maturation. It includes a group of proteins with similar structures. Inhibition of NF- $\kappa \mathrm{B}$ pathway has been shown to produce tolerogenic DC $[86,87]$. Silencing of RelB, which is a primary $\mathrm{NF}-\kappa \mathrm{B}$ protein, reduces the expression of MHC-II, CD80, CD86, and finally prevents DC maturation. RelB silenced DCs were able to inhibit antigen-specific alloreactive immune rejection, and decrease antigen specific $\mathrm{T}$ cell proliferation [88]. Significantly reduced allograft rejection was achieved after administration of RelB silenced donor DC [88]. Inhibition of the expression of p35, which is a subunit of IL-12, was an effective approach for antigen-specific immune modulation [89, 90]. DCs treated with siRNA against p 35 were able to induce potent $\mathrm{Th} 2$ deviation of antigen-specific response; reduce alloreactivity by effective inhibition of $\mathrm{T}$ cell proliferation; shift allogeneic $\mathrm{T}$ cell polarization from $\mathrm{Th} 1$ to $\mathrm{Th} 2$. In another study, Tol-DC was generated through the treatment of antisense ODN to inhibit the expression of CD80 or CD86. Allograft survival was prolonged by administration of modified DCs, which could induce T cell hyporesponsiveness and apoptosis of T cells [91]. In addition, silencing of CD80 or CD86 with siRNA inhibited T cell responses in mixed lymphocyte reaction (MLR), generated T regulatory cells [92], and improved cardiac allograft survival [93]. Tol-DC could also be generated through the silencing of CD40, which is another critical costimulatory molecules [94]. Silencing of CD40 gene expression in DCs resulted in increased IL-4 production, and decreased IL-12 production and allostimulation activity. The Th2 cytokine production from allogenic T cells was stimulated [95]. MyD88 is a key adaptor of toll-like receptor signaling [83]. It is critical for DC function and proinflammatory cytokine production. The silencing of MyD88 significantly improved the allograft survival [96]. Suppressor of cytokine signaling (SOCS) molecule is an 
intracellular inhibitor of Janus kinase. Silencing of SOCS gene in DC resulted in reduced allogeneic mixed leukocyte reactions. In addition, systemic administration of SOCS silenced DCs improved the survival of allograft in rat [97].

\subsubsection{Reducing immunogenicity of donor islets}

HLA is one of the primary causes of Th1/HLA class II antigen rejections in solid organ transplantation. Human islet also has an immunogenic potential, which depends on their HLA incompatibility with the recipient. The alloreactivity is correlated with acute rejection and graft failure. In HLA matching human islet transplantation, the alloreactivity is significantly reduced [98]. However, because of the limited sources of islet donor and extensive HLA polymorphisms, the availability of HLA-match donors is extremely limited. In addition, the fact that multiple donors being required to obtain a sufficient number of islets for transplantation makes the donor selection based on HLA more difficult. Therefore, HLA-unmatched islets are used to meet the clinical needs. These transplanted islets undergo aggressive immune rejections. Silencing of HLA expression provides a promising method to reduce the immunogenicity of HLAunmatched donor islets [99]. Enhanced resistance to allo-reactive T cell toxicity was observed in human cells treated with lentivirus expressing shRNA against pan class I and allele-specific HLA [100]. Silencing of HLA in solid organ transplantation was also reported $[101,102]$.

\subsubsection{Preventing ischemic reperfusion injury and apoptosis}

There are multiple challenges including ischemia/reperfusion, mechanical and enzymatic stresses during islet isolation and preservation processes. These factors can activate inflammation and intracellular stress signal pathways, and lead to the over-expression of proinflammatory mediators, such as proinflammatory cytokines, ROS, caspases, and transcription factors. The consequence is the loss of islet function and viability. In addition, further adaptive immune response against transplanted islets will also be triggered [103].

\subsubsection{Block the apoptosis}

During the isolation and preservation processes, islets are challenged with various stresses. After transplantation, there are multiple factors that could induce the islet cell death. The survival of transplanted islets could be improved by preventing islet $\beta$ cells apoptosis [64]. Caspases are the most important mediators of apoptosis, which include initiator caspases (caspase 8 and 9), and effector caspases (caspase 3, 7). Inhibition of caspase activity with caspase inhibitors, or anti-apoptotic genes, has been reported to prevent apoptosis of islets and improve their function [64]. The silencing of caspase 3 and/or caspase 8 was able to reduce renal and liver ischemia/reperfusion injury, which is a significant problem in organ transplantation [104-106]. Silencing of caspase 8, which is a mediator of extrinsic apoptosis pathway, could minimize Fas or proinflammatory cytokines-induced cell apoptosis. Silencing of caspase 3 (which is the converging point 
for both extrinsic and intrinsic apoptotic pathways) prevented $\beta$ cell apoptosis triggered by both extrinsic and intrinsic stimulations. We have demonstrated that the silencing of caspase 3 protects islets from inflammatory cytokine-induced apoptosis [107]. Delivery of caspase 3 siRNA to rat insulinoma (INS-1E) cells significantly reduced the number of apoptotic cells. Ex vivo transduction of human islets with adenoviral shRNA prior to transplantationreduced caspase 3 activity in islets and significantly improved islet function. All of the mice transplanted with adv-caspase 3 shRNA transduced islets achieved normoglycemia one day posttransplantation and maintained up to 17 days. In contrast, for mice transplanted with untreated islets, where normoglycemia was achieved in only $60 \%$ at day 1 , and $80 \%$ at day 4 post transplantation. Fas: Fas/FasL interaction is another important mechanism for triggering $\beta$ cell apoptosis [78]. Treating $\beta$ cells with siRNA-Fas significantly reduced caspase 3 activity and inhibited Fas-mediated $\beta$ cell apoptosis [108]. In other studies, Fas siRNA has been successfully used to reduce apoptosis in the liver $[109,110]$ and heart transplantation [111]. Burkhardt et al. used siRNA to silence Fas expression in murine insulinoma cells. Their results showed that siRNA against Fas inhibited cytokine-induced Fas mRNA expression and reduced cell surface Fas protein. However, due to the slow turn-over of Fas protein, a complete inhibition was not observed until prolonged incubation with siRNA. In addition, Fas siRNA effectively reduced cytokine-induced $\beta$ cell apoptosis as determined by caspase 3 activity and TUNEL assay [108].

\subsubsection{Prevent complement activation}

Downregulation of complement 3 or complement C5a receptor with siRNA has the potential to prevent renal ischemia-reperfusion injury [104, 112]. In another study, UW solution containing siRNA against complement 3 also showed the ability to protect donor organs in heart transplantation [111]. For islet transplantation, the complement pathway is activated during the ischemia-reperfusion injury and blood-mediated inflammatory reaction. siRNA targeting $\mathrm{C} 3$ and $\mathrm{C} 5 \mathrm{a}$ receptors might be a potential target for improving the viability of transplanted islets.

\subsubsection{Inhibit inflammatory pathway}

As discussed in the previous section, proinflammatory cytokines activate several inflammatory signal pathways, upregulate multiple key genes, and finally results in islet cell death. The major mediators in this process are potential targets for preventing islet cell death. iNOS gene: iNOS gene expression on islets increased significantly after transplantation. The maximal iNOS gene expression was observed one day after islet transplantation and then declined progressively [48]. Increased iNOS gene expression led to an early inflammatory process in transplanted islets. Nearly, $50 \%$ of the cytokineinduced genes are NO dependent, emphasizing the role of this radical in the late effects of cytokines on insulin producing $\beta$ cells [59]. Therefore, silencing of iNOS gene might reduce iNOS mediated inflammatory response and prevent islet cell death. McCabe et al. used lentiviral vector expressing shRNA to suppress IL-1 $\beta$-mediated induction of iNOS expression, resulting in significant protection against the cytotoxic effects of IL-1 $\beta$ exposure $[113,114]$. We designed siRNA to inhibit rat and human iNOS gene expression 
$[115,116]$. Due to the difference between human and rat iNOS mRNA sequences, different iNOS siRNA were designed for efficient gene silencing. A dose and sequence dependent inhibition of iNOS gene expression and NO production was observed in rat $\beta$ cell line (INS-1E cell) and human islets. iNOS gene silencing protected $\beta$ cells from inflammatory cytokine-induced apoptosis and preserved their insulin secretion ability. However, the effect of iNOS gene silencing on the apoptosis of islet was only moderate, as evidenced by $25-30 \%$ reduction in caspase 3 activity and in the percentage of apoptotic cells. Since an islet is a cluster of 200-1000 cells, the transfection efficiency of lipid/siRNA complexes on human islets was only $21-28 \%$, in contrast to the high transfection efficiency ( $>90 \%$ ) in $\beta$ cell lines. Therefore, to achieve satisfactory protective effects on human islets, we need to improve the transfection efficiency, which could be achieved by the rationale design of vectors $[107,117]$ or modifying the delivery approaches. This will be discussed in the later sections.

Transcription factor NF- $\mathrm{kB}$ plays an important role in the inflammation and ischemiareperfusion injury. For example, several genes are putative targets of NF- $\mathrm{kB}$ and their expression levels are changed after cytokine exposure [61]. Transcriptional activity of $\mathrm{NF}-\kappa \mathrm{B}$ is related to the expression of proapoptotic genes in cytokine stimulated $\beta$ cells [62]. Recombinant adeno-associated virus (AAV) encoding shRNA against NF- $\kappa B$ RelA (p65) subunit was evaluated to reduce NF-kB mediated inflammation [118]. The expressed shRNA against RelA significantly reduced p65 protein expression and suppressed IL-8 secretion in a cellular TNF- $\alpha$-induced inflammation model. In another study, siRNA against NF- $\kappa B$ RelB significantly attenuated ischemia-reperfusion injuryinduced renal dysfunction and protected mice against lethal kidney ischemia [119].

\subsection{Method of Gene Silencing}

\subsubsection{Types of RNAi methods}

The applications of RNAi generally utilize two types of molecules: chemically synthesized short interfering RNA (siRNA) or vector-based short hairpin RNA (shRNA). Although siRNA and shRNA can be applied to achieve similar functional outcomes, they are intrinsically different molecules as discussed below.

\subsubsection{Chemically synthesized siRNA}

siRNA of 19-23 bp can be synthesized chemically in vitro. Advances in solid phase synthesis technique make it possible to produce siRNA with high purity and precisecontrolled sequence. Both sense and antisense strands are synthesized separately and annealed to form double stranded siRNA duplex. After being delivered into cytoplasm, siRNA is directly incorporated into RNA-induced Silencing Complex (RISC) to have its function. Several rate-limiting steps for vector-based shRNA are avoided in siRNA mediated gene silencing. Therefore, the gene silencing effect of siRNA is potent and can appear in a short time after transfection. This feature also makes the design of siRNA 
relatively simple, since the factors regarding short hairpin expression and process are not involved. However, the 2'-OH group in siRNA molecules makes them extremely unstable and susceptible to enzymatic degradation. Only transient gene silencing can be achieved with siRNA and it disappears within several days post transfection.

\subsubsection{Vector-based shRNA}

shRNA is an RNA sequence that makes a tight hairpin turn that can be used to silence gene expression via RNAi. Vector-based shRNA utilizes endogenous cellular machinery to function. After entering the cell nucleus, vector-based shRNA with stem loop structure is expressed to produce pri-shRNA in the nuclei, which is then processed into pre-shRNA by an enzyme named Drosha. Pre-shRNA is exported to the cytoplasm by exportin-5, where it is further processed by Dicer (an RNAse III enzyme) to produce functional siRNA [120]. siRNA is then incorporated into RISC. Activated RISC with antisense strand is formed by cleavage of sense strand. Activated RISC recognizes target mRNA with a complementary sequence, and result in the cleavage of target mRNA (perfect complementarities) or translation inhibition (not prefect complementarities) [24].

\subsubsection{Design elements of siRNA and shRNA}

\subsubsection{1. siRNA and shRNA sequences}

siRNA sequence is the most important factor that determines the level and duration of gene silencing. Because of high sequence specificity, gene silencing efficiency changes dramatically with minor alteration in siRNA sequence. Mutation in the antisense strand usually makes the activated RISC unable to recognize target mRNA. There are several web-based tools available for predicting effective siRNA sequences and empirical rules are incorporated in the siRNA design software to optimize siRNA sequence [121-124]. These rules includes: thermodynamic property [125], length of siRNA sequence [126], GC contents, RNA secondary structure, sequence region, single nucleotide polymorphism sites, avoiding repeats and low complex sequence, avoiding off-target effects on other genes or sequences $[127,128]$. siRNA sequences predicted by software, however, are not always very effective in silencing a target gene, and sometime the most potent siRNA sequence is missed. Practically, a potent siRNA can be selected through testing of several candidate siRNA sequences designed with bioinformatics tools.

Since shRNA works through the production of siRNA within the cells, there are plenty of common rules applicable to both siRNA and shRNA sequence design. Actually, people have used the siRNA designing software to predict shRNA sequence with success. Due to the similarity between siRNAs algorithm and that of shRNAs, it is not surprising to keep the gene silencing efficiency after converting siRNAs into shRNAs. However, some features are not required for siRNA but are essential for shRNA expression. For an example, strong GC preference at position 11 and the preference for AU but not GC at position 9 might be the different properties for shRNA sequences [125]. The incompatibility of siRNA design algorithm for shRNA design has also been reported 
[129]. It would be a good idea to design shRNA sequences using shRNA design algorithm. Similar to siRNA design, several shRNA sequences should be experimentally screened to find potent shRNA sequences. An obvious disadvantage of this approach is that the cloning of shRNA into plasmid is a time consuming process. Fortunately, predesigned shRNA plasmids for most genes are now commercially available. In most cases, potent shRNA sequences for a particular gene could be found by screening several predesigned shRNAs.

\subsubsection{Expression cassette for $\operatorname{sh} R N A$}

Although shRNA sequence design is critical for efficient gene silencing, it is equally important to have a proper shRNA expression cassette, which determines the magnitude, duration and specificity of shRNA expression. In addition, the structure of shRNA backbone also plays an important role in shRNA transcription, processing, and nuclear export. The effect of different promoters and shRNA structures has been extensively investigated to improve the level and duration of gene silencing.

Promoters refer to DNA sequences that provide a binding site for RNA polymerase and transcription factors. DNA transcription starts after transcription factor binding to promoter sequences and recruitment of polymerase. The level and duration of shRNA expression depends on the properties of promoters used. Three types of promoters, such as constitutive promoter, inducible promoters, and islet specific promoter will be discussed here.

The first type of promoters is constitutive promoters. There are three types of constitutive promoters used for shRNA expression, including polymerase III, polymerase II, and polymerase I promoters. U6 and $\mathrm{H} 1$ are the two most commonly used polymerase III (Pol III) promoters for shRNA expression. This is because Pol III promoters transcribe small, highly structured RNAs without poly (A) and 5'-cap in mammalian cells [130]. However, Pol II promoters such as CMV [131, 132], U1 promoter [133], and E1b promoter [134] are also used for shRNA expression. Particularly, CMV promoter can be used for coexpression of a cDNA and a shRNA with a single promoter or to drive the expression of a shRNA embedded in a miRNA structure [132]. Usually, a poly (A) signal is needed for the termination of CMV promoter expression. A modified CMV promoter can also be terminated by poly(T) termination signal [131]. To increase the activity of U6 or H1 promoter, hybrid promoters composed of CMV enhancer and U6 or H1 promoter were constructed for shRNA expression [135-137]. Polymerase I promoter has also been reported for shRNA expression [138], however, the use of pol I promoter is still not well investigated.

The second type of promoters is inducible promoter. High level constitutive expression of shRNA may saturate cellular machinery and cause cell toxicity. In addition, it is undesirable to constitutively silence genes that are essential for cell viability. Therefore, inducible promoters that can silence genes in a tunable pattern were developed. Different conditional RNAi strategies have been reviewed elsewhere [139]. Here, we list some examples to discuss the concept of using inducible promoters for gene silencing. 
Czauderna and coworkers [140] constructed tetracycline-inducible U6 or 7SK promoters by introducing a tetracycline operator (tet $\mathrm{O}$ ) sequence between TATA box and transcription start site. shRNA expression was induced by adding tetracycline which could dissociate the repressors from the tet O. Similarly, tetracycline inducible H1 promoter was also constructed to silence $\beta$-catenin [141]. An IPTG-H1 promoter has been developed by placing a lac operator between TATA box and transcription start site [142]. Reversible gene silencing was achieved with above expression systems and the gene silencing effect will disappear within 3-4 day after removing the inducers. An alternative way for inducible gene silencing is Cre-LoxP recombination system, which usually results in an irreversible gene silencing. In this system, a Lox-flanked stuffer sequence with termination sequence (five thymidines) is inserted between $\mathrm{H} 1$ promoter and transcription start site. After expression of Cre protein, the stuffer sequence is removed due to the recombination of two loxP sites and thus activate the promoter [143]. Hypoxia inducible promoter may have additional advantages for its application in islets, which are usually under the hypoxic condition after isolation. The use of hypoxia inducible promoter could restrict the gene or shRNA expression within hypoxic islets. Therefore, it could avoid unwanted gene expression and silencing on normoxic tissue or organs and prevent potential toxic side effects. Lee et al. constructed pRTP801-Luc or pRTP801-VEGF plasmids bearing a hypoxia inducible promoter. After transfection into rat islets, transgene expression was higher in hypoxic islets than those in normoxic condition [144, 145]. Although there is still little report about the use of hypoxia inducible expression cassette for gene silencing in islets, we believe that its application in islet gene silencing will increase in the future.

The third type of promoters is islet-specific promoter. Several islet-specific promoters have been tested to drive the specific transgene expression in islets. Among them, rat insulin promoters are being extensively investigated [108, 146, 147]. To improve the performance of rat insulin 2 gene promoter in isolated pancreatic islets, an expression cassette including $1.5 \mathrm{~kb}$ of the porcine INS 5' UTR and the 3' UTR of the bovine growth hormone gene was constructed, which showed robust and specific gene expression on islet $\beta$ cells [148]. Chai et al. have compared a series of rat insulin promoter sequences with various lengths. The highest promoter activities was observed in a modified RIP3.1 promoter including the non-coding regions in exon1, intron 1, and part of exon 2 of the rat insulin gene 1 as well as the insulin gene promoter region. This promoter showed 5-fold higher activity in INS-1E cells than a full-length RIP promoter or a CMV promoter. In addition, RIP3.1 promoter demonstrated $\beta$ cell specific gene expression and glucose responsive gene expression [146]. Recently, the promoter for pri-mir375 has been indentified, which could selectively express pri-mir375 in pancreatic islets [149]. Since miRNA-based shRNAs have been successfully used for shRNA expression, the finding of this promoter provided an option for islet specific shRNA expression.

shRNA backbone structure is also critical for effective gene silencing. High level of shRNA expression does not necessarily lead to efficient gene silencing, because nuclear export, dicer enzyme processing and other rate limiting steps are also critical for efficient gene silencing. These processes are greatly influenced by the shRNA structure, which includes the stem length, loop sequence, the sequence of flanking regions. A typical 
shRNA is composed of a 19-21bp sense sequence and a 19-21bp antisense sequence linked with 6-9bp loop sequence. A termination sequence of 5-6 thymidines is usually included at the end of stem-loop structure, except for shRNA driven by a pol II promoter. Various shRNA structures have been studied to improve gene silencing efficiency. Siolas et al. found that synthetic 29-mer shRNAs were more potent than 19-mer shRNAs, while the loops sequence did not have any effect [150]. In contrast, Jeanson-Leh et al. reported that increase in the stem length from 19-mer to 29-mer did not show any improvement in gene silencing [151]. It was also reported [125] that gene silencing of shRNAs with 19mer stem was better than those of shRNA with 29-mer stem in the context of a 9-bp loop.

An emerging trend in shRNA design is to incorporate siRNA into a miRNA precursor backbone so that it can be processed efficiently by intracellular miRNA machinery. mir30-based shRNA has been reported to be more efficient than conventional shRNA [152-154]. Boden et al. designed a shRNA against HIV-1 transactivator protein TAT with a mir30 backbone, which showed $80 \%$ more potency in silencing target gene than a conventional shRNA [152]. In another study, Li et al. compared 14 pairs of conventional shRNA and mir30-based shRNA against luciferase gene, and 10 pairs of conventional shRNA and mir30-based shRNA against the mouse tyrosine gene. Results indicated that in 11 out of 14 pairs of shRNA against luciferase and all of those for mouse tyrosine gene, a better gene silencing was observed in conventional shRNA rather than in mir30based shRNA [125]. Boudreau et al. have also demonstrated that optimized shRNA are more efficient than mir30-based shRNA in silencing of several genes, including green fluorescent protein (GFP), spinocerebellar ataxias, and Huntington disease [155]. In our own studies, we also found that mir30-based shRNA against human iNOS genes was less potent compared with conventional shRNA [117]. These discrepancies indicate that current understanding of shRNA design is still limited and further exploration is necessary to develop a satisfactory rule for shRNA design. The use of miRNA backbone for shRNA expression also has the potential to reduce toxicity [156] and to have tissue or cell type specific gene silencing [157].In addition, we could also construct pri-miRNA cluster, in which multiple miRNA-based shRNA is driven by a single promoter to form a single transcript $[158,159]$.

Recently, several other shRNA structures have been investigated for different applications. In one study, a tRNA-shRNAs chimeric expression cassette was constructed, in which a tRNA promoter was used to drive the expression of shRNA linked to the 3' acceptor stem of tRNA [160]. This system could achieve similar or even high gene silencing than shRNAs expressed from U6 or H1 promoters [160-162]. Since exportin-t, but not exportin-5, was used for the nuclear export of tRNA-shRNAs, it could reduce toxicity related to exprortin-5 saturation $[161,163]$. Tandem siRNAs: Zheng et al. designed a dual promoter system (pDual) by inserting siRNA sequence between two promoters (including mouse $\mathrm{U} 6$ and human $\mathrm{H} 1$ promoters) with opposing directions [164]. 


\subsubsection{Off-target effects}

Since the first report by Jackson et al. in 2003 [165], many studies have shown that both siRNA and shRNA could cause off-target effects. From a therapeutic standpoint, the offtarget effects should be avoided to prevent unintended effects. Therefore, understanding the mechanisms of off-target effects and developing strategies to enhance RNAi specificity is critical for the application of RNAi. There are two types of off-target effects: specific off-target effects, which are caused by the partial sequence complementarity between mRNA and siRNA, and non-specific off-target effects, which are the outcome of overall translation inhibition due to the activation of immune system and cellular toxicity. The mechanism of off-target effects was reviewed extensively elsewhere $[166,167]$.

One type of off-target effecs is specific off-target effects. Silencing of non-target genes is possible if there is as little as 8-nt homology at the seed region sequence [168]. The offtarget effects may also be observed when mRNA shared 7-nt homology with the guide siRNA sequence [169]. However, not all the mRNA with this level of homology will be silenced and it depends on the location of complementary region within siRNA and the mRNA. Seed region is the nucleotides $2-7$ at the $5^{\prime}$ end of siRNA. Complementarity between the siRNA seed region and the 3' UTR of mRNA is an important predictor for off-target effects [168]. Several approaches have been developed to avoid the specific off-target effects. It could be prevented by designing siRNA sequences to minimize the complementarity between seed region of siRNA and the 3' UTR of mRNA [170]. One or two base mismatch between siRNA and target mRNA are usually tolerated without losing gene silencing efficiency [171]. This allows us to modify the siRNA sequence to minimize the complementarity with 3'UTRs of the untargeted mRNAs. However, the modification of siRNA sequence is associated with the risk of losing gene silencing efficiency. Since the passenger strand is not involved in the recognition with target mRNA, modifications of passenger stand is preferred and more tolerant. For example, breaking a passenger strand into two segments significantly reduced off-target effects [172]. RNA-DNA chimeras [173] have also been developed to avoid off-target effects.

Another type of off-target effects is nonspecific. The innate immune systems could be activated by several elements including dsRNA, plasmid or viral vectors, cationic delivery carriers. The activation of immune system causes the inhibition of global gene expression and false positive gene silencing effect. There are multiple receptors involved in the activation of immune system. They are cytoplasmic receptors, including dsRNAdependent protein kinase R (PKR), retinoic acid-inducible gene-1 (RIG-1), and melanoma differentiation-associated gene-5 (MDA-5) [174-176] and endosomal toll-like receptors (TLRs) [177, 178]. For an example, introduction of long dsRNA into cells leads to the activation of PKR , and results in the activation of innate immune system and typeI IFN production [176]. Although the use of shorter siRNAs could minimize the activation of immune response, sequence dependent activation of TLR7 and 8 could not be eliminated [179]. For vector-based shRNA, siRNA expressed inside the nucleus and exported into the cytoplasm. The use of vectors-based shRNA could thus avoid the activation of TLR3, which recognize dsRNA. However, the plasmid vectors will activate 
TLR9, unless the unmethylated CpG motifs were removed from the vectors [180,181]. High levels of siRNA and shRNA could also compete for intracellular miRNA/shRNA process machinery. The saturation of epxortin-5 and RISC component, Argonaute- 2 will cause cell toxicity [182]. This can be minimized by designing of proper expression cassettes to carefully control shRNA expression levels and avoid the saturation of miRNA machinery.

\subsection{Combinatorial RNAi}

Due to the complexity of molecular mechanism of islet cell death, it is almost impossible to effectively prevent islet cell apoptosis by silencing the expression of a single gene or over-expression of a single protective gene. Therefore, combinatorial RNAi strategies which could synergistically work on multiple targets are required to achieve satisfactory outcomes. This could be realized by simultaneous knockdown of different genes involved in multiple biological processes or signal pathways. Alternatively, we could also simultaneously silence certain harmful genes and at the same time over-express protective genes. Different strategies for combinatorial RNAi will be discussed (Figure 2.4).

\subsection{1. $\quad$ siRNA pool}

The use of several siRNAs target different regions of a single gene is more efficient than the use of a single siRNA sequence in reducing gene silencing. Cheng et al. tested a pool of two siRNA sequences against TGF- $\beta 1$ gene in HSC-T6 cells [183]. In that study, the siRNA pool showed better inhibition of TGF- $\beta 1$ protein expression as determined by western blot analysis. In addition, an enhanced inhibition of the expression of downstream genes including type $\alpha 1$ (I) collagen and $\alpha$-smooth muscle actin ( $\alpha$-SMA) were also observed. In another study, Chen et al. used siRNA pool targeting different regions of human hepatitis B surface antigen. The treatment of this siRNA pool showed efficient inhibition of hepatitis B surface antigens production at HBV-producing HepG2.2.15 cells [184]. Alternatively, co-silencing of multiple genes with siRNAs has also been used to achieve synergistic effect. For an example, siRNA against three different genes including TNF- $\alpha$, complement 3 (C3) and Fas were tested to reduce ischemia/reperfusion injury to donor organs. The siRNAs used in this study, were supposed to silence several different genes involved in apoptosis, complement activation, and inflammations [111]. In other studies, combination of siRNAs targeting complement 3 and caspase 3 [104] or siRNAs targeting caspase 3 and caspase 8 [105] have been used to prevent renal ischemic injury. siRNA pool targeting mRNAs of multiple genes will be suitable for improving the islet viability because of the involvement of multiple processes in islet damage. 


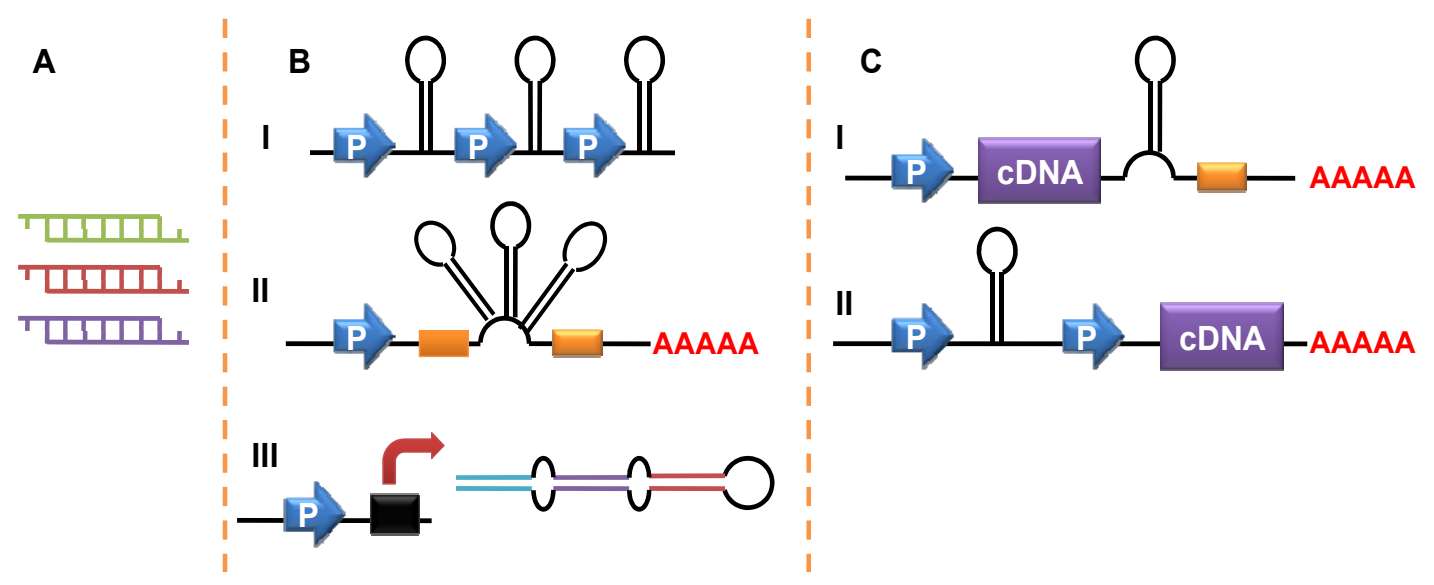

Figure 2.4 Combinatorial RNAi strategies for islet genetic modification.

(A) siRNA pool targeting different sites of a single gene or targeting multiple genes. (B) Co-expression of multiple shRNAs, (I) multiple shRNAs under separate promoters, (II) miRNA cluster mimics, (III) long hairpin RNA. (C) Co-expression of shRNAs and cDNAs, (I) from a single miRNA backbone, (II) from separate promoters. 


\subsubsection{Co-expression of multiple shRNAs}

There are several scenarios for co-expression of multiple shRNAs from a single vector: (1) multiple shRNAs with the same sequence, (2) multiple shRNAs against different regions of the same gene, or (3) multiple shRNAs against different genes. For the first one, the purpose is to increase the intracellular shRNA levels. However, it is rarely used because the shRNAs levels could be controlled more conveniently by selecting proper promoters. The use of multiple shRNAs against different regions of a single gene is believed to be more efficient in reducing target gene expression levels, possibly due to the synergistic effect. The advantage of using multiple shRNAs against different genes is the possibility to block multiple mediators in the signal pathways. Co-expression of multiple shRNAs were widely studied as an antiviral treatment modality [185]. Although the disease targets are different, the shRNAs expression strategies could be applied to genetic modification of islets. Here, some strategies for co-expression shRNAs will be reviewed.

Gonzalez et al. constructed a vector where multiple shRNAs against HLA class genes were under the control of a common U6 promoter. A dose dependent increase in RNAi efficiency was achieved though raising shRNA copy numbers up to six [186]. Several other studies demonstrated the feasibility of co-expressing multiple shRNAs by utilizing miRNA backbone. In these studies, strong promoters (such as a CMV promoter or a unbiquitin $\mathrm{C}$ promoter) were used to control the expression concatemerized multiple miRNA (miR30 or miR155)-based shRNAs [158, 187-189]. However, the increase in shRNA copy number does not necessary improve gene silencing efficiency. Further investigation will help us to understand the optimal hairpin numbers, positions, backbone sequences required for efficient RNAi.

Long hairpin RNA (lhRNA) [190, 191] or extended hairpin RNAs (e-shRNAs) [192, 193], also showed the potential to co-express several siRNAs. For an example, Liu et al. constructed a vector with e-shRNAs containing two siRNAs and found that a stem length of $43 \mathrm{bp}$ is the minimal requirement. The $66 \mathrm{bp}$ was the minimal length needed to produce three siRNAs [193]. Instead of expressing multiple shRNAs, a single hairpin with multiple siRNAs was expressed in this type of construct. Different levels of gene silencing were observed among these siRNAs and were dependent on their position to the stem loop. The siRNA most close to the loop showed comparable gene silencing to single shRNA, however, other siRNAs usually showed less gene silencing efficiency [191, 193]. In addition, three siRNA units is the upper limit for one construct and the gene silencing efficiency was significantly reduced when four siRNA units were included within one construct [193].

Alternatively, it is also possible to express shRNAs from two or more independent promoters. For example, two shRNAs against different isoforms of glycogen synthase kinase 3 gene were under the control of separate U6 promoters and achieved efficient inhibition of target gene with additive effect [194]. A similar study showed the use of separate U6 promoters to control the expression of shRNAs targeting different Smads 
[195]. Co-expression of multiple miRNA-based shRNAs against different gene has also been reported [196].

\subsubsection{Co-expression of shRNAs and cDNAs}

We have recently demonstrated the beneficial effects of co-expressing multiple genes from a single vector for improving the outcome of islet transplantation. The combination of growth factor and anti-apoptotic genes showed synergistic effect in protecting transplanted islets $[18,19]$. A vector could co-express both siRNA and cDNA will be particularly interesting [197]. Not only is the inhibition of harmful genes but also overexpression of protective genes is needed to protect islets. We constructed vectors for coexpression of shRNAs against iNOS genes and a VEGF cDNA. In this vector, VEGF was designed to promote the revascularization of transplanted islets. The shRNA against iNOS were designed to reduce the expression of iNOS genes and minimize iNOS mediated islet cell death. In this study, we also investigated the use different expression cassettes for co-expression of shRNA and cDNA from a single vector. The plasmid vector contained two expression cassettes, where VEGF was under the control of a strong CMV promoter and shRNA was driven by U6, H1, or CMV promoters. Alternatively, it was also possible to express cDNA and shRNA from a single expression cassette. In this case, we used CMV promoter to drive the expression of miRNA-based shRNA and VEGF cDNA was inserted between the CMV promoter and shRNA. This strategy was reported previously by Qiu et al., where a miRNA-based shRNA was placed in the 3'UTR of a red fluorescent protein (RFP) for co-expression of both shRNA and RFP [198]. Similar study was also reported by Shin et al., where a GFP gene was inserted between the Tet response element promoter and miR30-based shRNA. This construct allows the co-expression of both GFP and shRNA [196]. In our study, both monocistronic and bicistronic systems showed high VEGF expression and efficient reduction of iNOS gene expression. These vectors could be further modified by replacing VEGF cDNA with another cDNA such as hepatocyte growth factor (HGF), which will enhance angiogenesis and promote $\beta$ cell proliferation. The shRNA against iNOS genes could be replaced various other shRNA targeting proapoptotic and inflammatory genes.

\subsection{Delivery Strategies for Enhanced Gene Silencing}

Although good progress has been made since the discovery of RNAi technique, the application of RNAi for therapeutic purposes is still limited, because of several biological barriers for siRNA delivery [199]. Systemic delivery of siRNA is required to block host against islet graft immune rejection. However, siRNA face several biological barriers after systemic administration [199], which usually result in the degradation of siRNA. Another potential issue for systemic siRNA delivery is the lack of its targeting ability, which requires the use of targeted delivery system to avoid gene silencing on other tissues or organs. In addition, the systemic administration will possibly induce the immune reaction not only by the siRNA but also by the delivery system, especially, when a viral vector is used. Alternatively, ex vivo delivery is an ideal approach for genetic 
modification of islets prior to transplantation. Since after isolation, islets are usually preserved in storage solutions before transplantation, we can transfer siRNA into islets prior to transplantation. This ex vivo siRNA delivery approach can also be used to generate immune tolerant dendritic cell (Tol-DC).

The design of proper delivery systems is critical for the clinical application of RNAi technology. Both non-viral and viral vectors have been widely used. The main advantage of viral vectors is their high transduction efficiency. However, the use of viral vectors is undermined due to safety issues and also the construction of individual viral vector is a time consuming process. Alternatively, non-viral approaches, which could avoid the problems for viral vectors, have become a promising approach for gene silencing. In this section, we will discuss siRNA delivery systems applicable to islet transplantation (Figure 2.5). Firstly, we will discuss the possibility of using free siRNA and chemically modified siRNA. Secondly, we will visit the cationic lipids or polymers for siRNA delivery. Thirdly, the use of bioconjugation will be discussed, which could enhance siRNA delivery into the cells and increase siRNA stability. Finally, we will summarize some viral vectors used for gene silencing including adenovirus and lentivirus.

\subsubsection{Free siRNA}

Free siRNAs are difficult to be transfected into the cells mainly because of their poor cell membrane permeability and sensitive to nuclease mediated degradation. However, it is still possible to deliver free siRNAs to islets with some physical approaches, such as hydrodynamic injection [200], in situ perfusion [201], and microporation [202]. Zheng et al. protected donor organs for heart transplantation with siRNA-containing solution [111]. In that study, heart graft from BALB/C mice were preserved in solution containing siRNAs against several genes involved in ischemia/reperfusion injury. This treatment resulted in efficient silencing of target genes in the grafts and lead to improved graft function. Due to the similarity among different organ transplantations, this approach should be equally applicable for islet transplantation.

However, native siRNA is degraded rapidly due to nuclease attack, therefore, chemical modification of siRNA has been extensively studied to (1) minimize nuclease degradation; (2) avoid innate immune system activation; and (3) reduce off-target effects [203, 204]. Since most siRNAs were produced through chemical synthesis, it is technically possible to incorporate various modifications into siRNA backbones (Figure 2.6). These modifications could be applied to phosphodiester or ribose. Non-bridging oxygen in the phosphodiester could be replaced with sulfur (phosphorothioate), boron (boranophosphate), or methyl (methylphosphonate) groups. The 2'-position of the ribose could also be modified to improve the stability of siRNA. The modification of ribose includes 2'-O-methyl (2'OMe), 2'-fluoro(2'-F), 2'-O-fluoro- $\beta$-D-arabinonucleotide (FANA), 2'-O-(2-methoxyethyl) (MOE), and locked nucleic acid (LNA), which contains a methylene bridge connecting the 2'-O with the 4'-C of the ribose ring. Single or combination of various types of modifications could be used depending on the aim of modification, which will be briefly discussed below. 


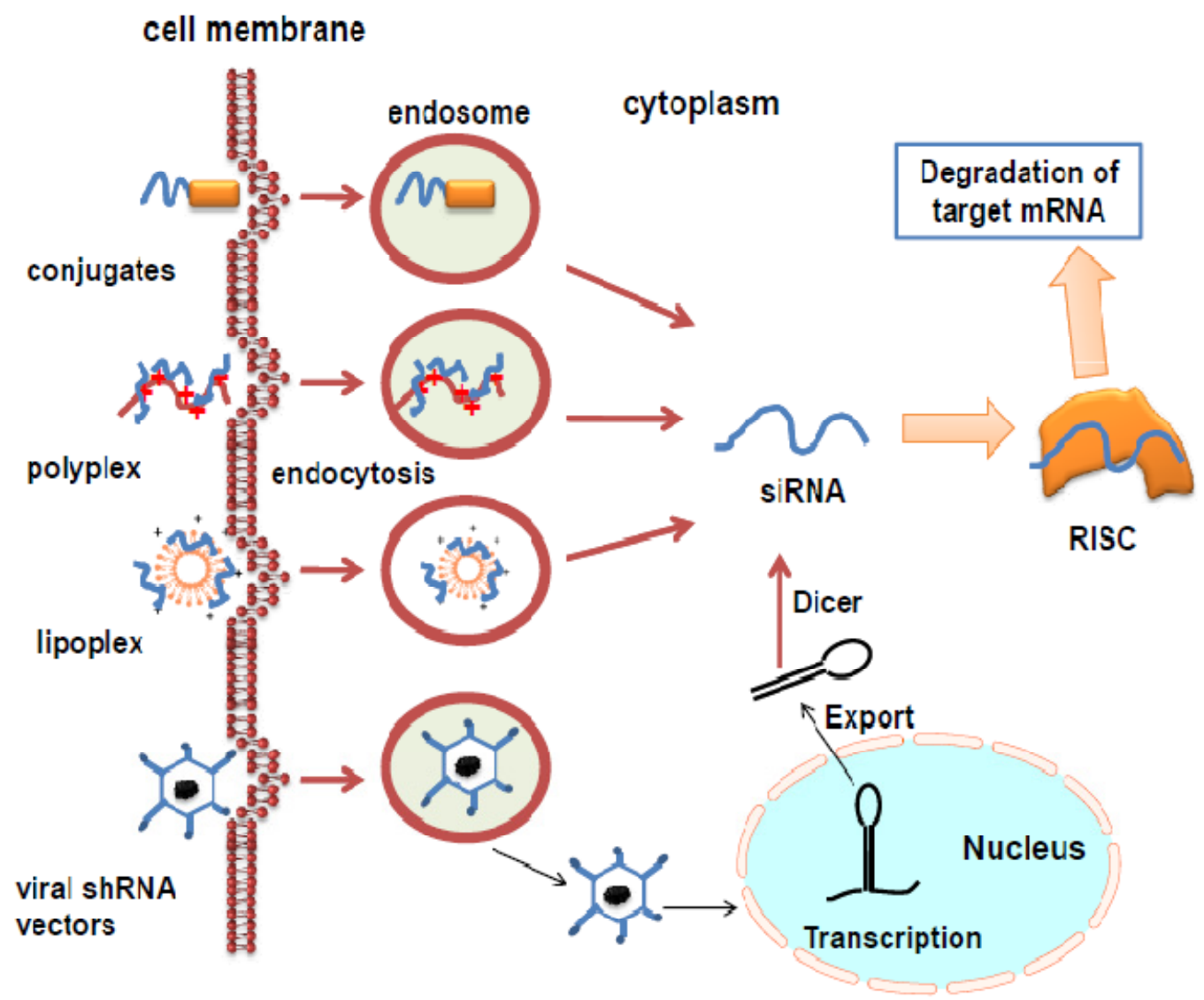

Figure 2.5 Delivery strategies for gene silencing.

Cationic liposomes and polymers are commonly used as transfection reagents for siRNA and shRNA. To avoid the use of cationic carriers, siRNA can also be conjugated to polymers such as poly(ethylene glycol) carrying targeting ligand(s). For enhanced and/or prolonged gene silencing, shRNA is often cloned into a viral (adenovirus, lentivirus, or adeno associate virus) vector. After administration, these delivery systems enter the cells mainly through endocytosis. Vector-based shRNA is further translocated into cell nucleus, where shRNA is expressed to produce pri-shRNA, which is further processed into pre-shRNA by an enzyme named Drosha. Pre-shRNA is exported to the cytoplasm by exportin-5, where it is processed by Dicer (an RNAse III enzyme) to produce functional siRNA. For siRNA bioconjugation or siRNA complex, the escape and release of free siRNA from endosome into cytoplasm is an essential step. Finally, siRNAs will be incorporated into RNA-induced silencing complex (RISC) and guide the recognition and degradation of target mRNA. 
A. Modification of non-bridging oxygen internucleotide linkage
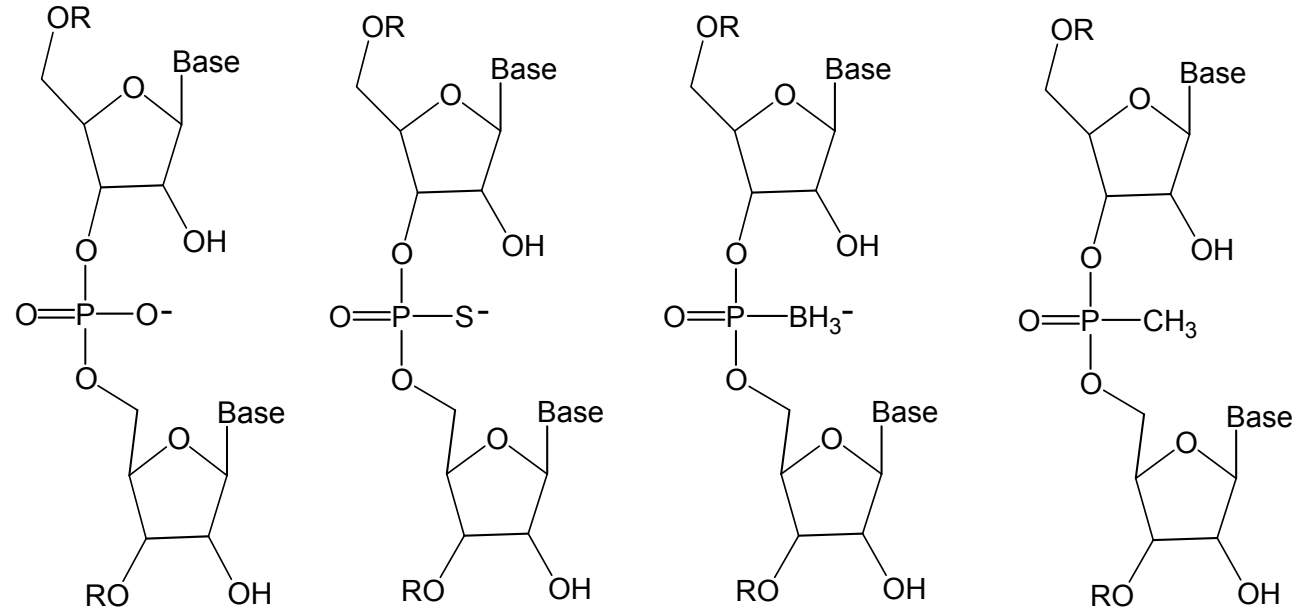

Phosphodiester phosphorothioate

boranophosphate

methylphosphonate

B. Modification of the sugar unit

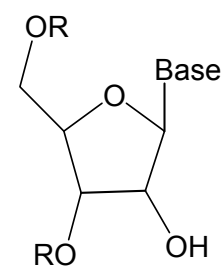

RNA

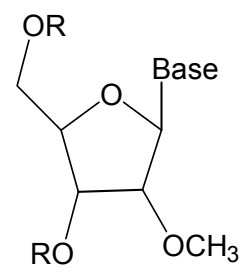

2'-O-Me

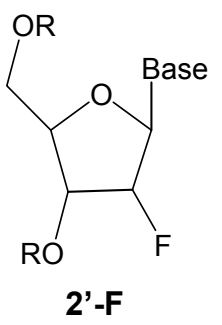

2'-F

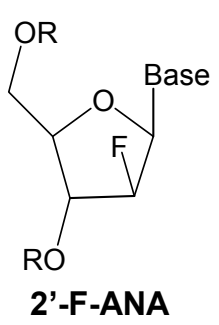

2'-F-ANA

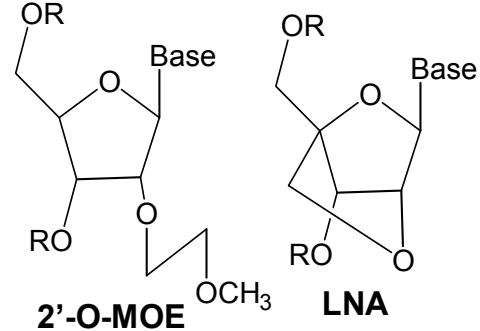

\section{Figure 2.6 Common modification introduced to siRNAs.}

(A) Modification of non-bridging oxygen internucleotide linkage. (B) Modification of the sugar unit. 


\subsubsection{Enhancing serum stability}

Unmodified naked siRNAs are extremely unstable with a serum half-life of less than 5 $\min [205,206]$, which restricts their clinical application. Thus, chemical modification of siRNAs has been extensively studied to improve the resistance of siRNAs to nuclease degradation. Theoretically, all above mentioned modifications can be applied to increase the serum stability of siRNA. The modification strategies should be carefully designed, since extensive modification of siRNAs usually leads to decreased potency [207-209]. Alternatively, selective modification of siRNAs at minimal position has been demonstrated to significantly improve the serum stability, while keeping the gene silencing potency. The 2'-O-Me or 2'F-RNA modification have been applied to the termini of the strands or a pyrimidine nucleotide which are vulnerable position for nuclease cleavage $[24,207,210]$.

\subsubsection{Reducing innate immune system activation}

As we discussed previously, siRNAs will activate the innate immune system and result in non-specific off-target effects. Chemical modifications can also be used to avoid or minimize siRNA-mediated activation of immune system. For an example, 2'-O -Me modification of siRNA was able to minimize the immune system activation [211]. Since the 2'-O-Me group is a competitive inhibitor of TLR7, minimal number of 2'modification is sufficient to inhibit the TLR-7 mediated immune stimulation [212].

\subsubsection{Reducing off-target effects}

Chemical modification could be applied to reduce specific off-target effects through minimizing the unwanted participation of siRNA in miRNA pathways. For an example, modification of the +2 position with 2'-O-Me on the antisense strand effectively reduced seed region mediated off-target effects [168]. The seed region could also be replaced entirely with DNA residues to reduce off-target effects while preserving the gene silencing potency [173].

\subsubsection{Complex formation}

Both cationic liposomes and polymers are widely used to form complexes with siRNA or plasmid shRNA through electrostatic interaction between positive charged carriers and negatively charged nucleic acids. Complex formation helps to condense siRNA or plasmid DNA and thus facilitate their cellular uptake.

Cationic lipids: Since the introduction of lipofectin at late 1980s [213], many cationic liposomes have been tested for delivery of plasmids, and recently for the delivery of siRNA. There are several commercial cationic lipid formulations for siRNA delivery, including lipofectamine 2000, oligofectamine, lipofectamine, RNAifect, and Fugene HD. We have tested lipofectamine to transfect a plasmid encoding an enhanced green fluorescent protein gene, pCMS-eGFP into intact human islets. However, only low 
transfection efficiency was observed, this is due to the fact that human islets are a cluster of around 1000 non-dividing cells [16]. In our further studies, a novel lipid with methylsulfonic acid in the cationic head group region to enhance lipid/DNA interaction was synthesized, which showed higher transfection efficiency than those of lipofectamine in intact human islets [214]. In contrast to plasmids, siRNA is efficiently transfected into human islets as well as INS-1E rat $\beta$ cell line. In this study, we used lipofectamine 2000 to transfect fluorescein-labeled siRNA. The transfection efficiency was determined by flow cytometry analysis. There was increase in transfection efficiency with increase in siRNA concentration, with maximum transfection efficiency of $95.9 \%$ and $28.3 \%$ on rat $\beta$ cell line and intact human islets, respectively [115]. This demonstrated the feasibility of in vitro genetically modifying human islets with siRNA/lipid complex.

Cationic polymers: In addition to cationic lipids, cationic polymers are also being used for plasmid or siRNA delivery. Although we could achieve decent in vitro transfection efficiency by optimizing transfection conditions, the cellular toxicity of cationic carrier is a major concern for its therapeutic applications. Furthermore, the poor intracellular dissociation of siRNA/polycation complex may reduce the intracellular bioavailability of siRNA. Recently, several biodegradable carriers have been developed to reduce toxicity and improve transfection efficiency. Here, we focus on the some recent progresses, rather than a comprehensive review of the use of cationic polymer for siRNA delivery which was extensively discussed elsewhere [215].

Reducible biodegradable carriers are designed based on the different redox potential between intra and extra cellular environment. The disulfide bonds in these carriers are usually stable enough to keep the integrity of the carrier and protect siRNA before cellular uptake. After cellular uptake, the disulfide bonds are cleaved, in response to the reducing intracellular environment. This will result in the degradation of carriers and release of siRNA. Reducible polymers with disulfide bonds have been investigated for delivery of both plasmid and siRNA [216-222]. The advantage of using reducible polymers is obvious, which not only reduced carriers associated toxicity, but also enhanced dissociation of siRNA from polycation/siRNA complex and thus improved gene silencing efficiency. The performance of these carriers could be further improved by incorporation of endosomal escaping moieties, such as histidine [220], and protonatable pendants [221]. Since the endosomal release of siRNA is a significant barrier for siRNA and gene delivery, those components which could facilitate "endosomal escape" will greatly enhance the gene silencing efficiency.

Poly (amino ester) (PAE) is another type of biodegradable polymers which has been extensively studied for gene delivery. Green et al. generated a library of PAE and determined parameters such as polymer type, polymer weight, DNA loading, and other biophysical properties. The leading PAE carrier found in their studies were better than jetPEI and lipofectamine2000 [223]. PAE has also been successfully used for siRNA delivery into lung cancer cells [224], hepatoma cells and primary hepatocytes [225], and fibroblasts [226]. Besides, several other biodegradable polymers can also be used for siRNA delivery, including polyphosphoesters [227-229], poly(2-aminoethyl ethylene 
phosphate) [230], poly(ethylene glycol)-peptide copolymers [231] and ketalized polyethylenimine [232].

\subsection{3. $\quad$ siRNA bioconjugation}

Bioconjugation of siRNA with lipids, polymers or other molecules will help to enhance its cellular uptake, systemic stability, and targeted delivery to specific cells. Technically, siRNA could be modified at the 5' or 3' terminus of sense strand or antisense strand. Because of the pivotal role of antisense strand in gene silencing, the modification of sense strand is preferred. Both cleavable and non-cleavable bonds can be used for siRNA conjugation. Acid-sensitive or reducible disulfide bonds are the most commonly used, because of the enhanced release of siRNA from conjugation inside the cell. It could avoid potential negative effects of bioconjugation and provide more amount of free siRNA available for gene silencing.

The molecules used for conjugation with siRNA determine the properties of siRNA conjugate. Attachment of lipids usually increases the hydrophobicity of siRNA, which are highly hydrophilic macromolecules. Lipophilic molecules such as cholesterol [233], bile acid and long chain fatty acid [234, 235], and vitamin E [236] could be conjugated to siRNA. After systemic injection, siRNA-lipid conjugate binds with lipoproteins depending on the degree of hydrophobicity. The interaction between siRNA-lipid conjugate and lipoproteins, lipoprotein receptors and transmembrane proteins has a great influence on the biodistribution and cellular uptake of siRNA after intravenous injection [234]. For an example, siRNA bound to low density lipoprotein (LDL) is mainly delivered to the liver, while a broader distribution was observed for siRNA binding to high density lipoprotein (HDL).

Conjugation of siRNA with cell-penetrating peptides (CPPs) such as TAT [237, 238], penetratin [238] and Transportan [239], has also been studied for siRNA delivery. siRNA-peptide conjugates show a cellular uptake as efficient as that achieved by cationic lipids and resulted in efficient silencing of target genes. However, conjugation of siRNA with peptides does not improve the in vivo stability of siRNA [238]. In addition, innate immune response could be activated after intratracheal administration of pentratin-siRNA conjugate [238], may be due to the immunogenicity of peptide.

To increase the systemic stability of siRNA, poly(ethylene glycol) (PEG) has been conjugated to siRNA by several research groups. In this type of conjugation, siRNA can be linked with PEG through disulfide bond or B-thio-propionate linkage. Polyelectrolyte complex micelles (PEC micelles) were formed between PEG-siRNA and cationic peptide (KALA) [240] or cationic polymers, such as polyethylenimine (PEI) and poly(L-lysine) (PLL) during the application of siRNA-PEG conjugate. A core was formed between negatively charged siRNA and polycation through condensation, while PEGs are surrounding the core to form a hydrophilic shell. This special core-shell structure can effectively protect siRNA against enzymatic degradation; prevent self-aggregation between PEC micelles, and increase the circulation time after systemic administration. 
This delivery system can efficiently silence VEGF gene expression in PC-3 cells in vitro [240, 241]. After intravenous injection, enhanced distribution of PEC micelles in tumor was observed, which resulted in significant reduction in VEGF expression in tumor and suppressed tumor growth [242]. siRNA-PEG PEC can be further improved by conjugation of lactose moiety at the distal end of PEG (siRNA-PEG-Lac). This will confer targeting ability to this carrier. The cellular uptake of siRNA in hepatoma cells was enhanced via receptor mediated endocytosis [243,244]. Delivery of siRNA against RecQL1 gene with lac-PECs inhibited muticellular HuH-7 spheroids growth for up to 21 days, while almost no effect was observed with oligofectAMINE/siRNA lipoplexes. It is probably due to the facilitated cellular uptake of Lac-PECs into the spheroids cells [244].

In addition to the enhanced delivery, siRNA are also conjugated with probes or dyes for bioimaging [245]. Multiple component siRNA conjugate was prepared which includs iron oxide core coated with crosslinked dextran coat, cy5.5 dye, as well as siRNA. After in vitro incubation, this conjugate was efficiently taken up by murine islets. The celluar uptake of siRNA was observed by magnetic imaging of iron core and near-infrared imaging. This study showed the posibility to integrate gene silencing and imaging components into a single conjugate to create a multiple function siRNA conjugtes.

\subsubsection{Viral vectors}

As naturally evolved vehicles, viral vectors utilize the virus infection mechanism for efficient gene transfer into host cells. Although safety concerns retarded the clinical use of viral vectors, their application in genetic modification of islets has the potential to improve the outcome of islet transplantation. This is mainly because of their high transduction efficiency compared with non-viral vectors. Here, we will discuss the properties of some commonly used viral vectors and their application in islet transplantation.

\subsubsection{Adenovirus}

Adenovirus is non-enveloped DNA virus, which could infect both dividing and nondividing cells. Recombinant adenovirus derived from adenovirus serotype 5 is commonly used for gene delivery. E1 region, which is essential for virus replication, is usually deleted to make replication-deficient adenovirus. In addition, adenoviral genome will not integrate to the targeting cells; therefore, a transient gene expression is achieved by adenoviral vector. The advantages of adenovirus include capacities to carry large DNA insert, easy to produce high titer virus, and its ability to infect non-dividing cells with high efficiency and high gene expression level.

We and others have used replication deficient adenoviral (Adv) vectors for ex vivo transduction of genes to islets to reduce the immune attack, prevent apoptosis, and promote the revascularization of transplanted islets $[15,18,19,64,107,117,246]$. Bain et al. first reported the feasibility of using Adv vector for gene silencing in islets and $\beta$ cell lines [247]. Adv vectors expressing siRNA against GLUT2 gene was used in their 
study to transduce rat islets. Maximum reduction in GLUT2 gene expression was achieved 3 days post transduction with $>90 \%$ at both mRNA and protein levels. After then, $\mathrm{Adv}$ vectors have been used to silencing different genes in islets to study the function of genes, such as synaptotagmin 9 [248], pyruvate carboxylase [249], tissue factor [250], and proislet amyloid polypeptide [251]. The maximum silencing efficiency was varied from $35 \%$ to $75 \%$ and achieved 3-4 days post-transduction. Gene silencing efficiency is determined by several factors: (1) the properties (turnover time, abundance) of target gene mRNA and protein; (2) the design of siRNA targeting sequence as well as expression cassette.

Since caspase 3 played an important role in islet $\beta$ cell apoptosis induced by different factors, the inhibition of caspase activities was a potential approach to improve the graft survival [64]. Recently, we determined the level and duration of caspase 3 gene silencing after transfection of caspase 3 siRNA into INS-1E cells and demonstrated transient gene silencing which did not last beyond 3 days. In an attempt to enhance the level and duration of caspase 3 gene silencing, we then constructed Adv vectors expressing shRNA against caspase 3 (Adv-caspase-3-shRNA) and determined their gene silencing efficiency in human islets. This adenoviral vector showed efficient caspase 3 gene silencing, which became more significant on day 5. Further, Adv-caspase 3-shRNA mediated caspase 3 gene silencing was able to counteract inflammatory cytokine-induced islet cell apoptosis in vitro. Taking care of these encouraging in vitro results, we then transduced human islets with this advenoviral vector prior to transplantation under the kidney capsules of diabetic NOD-SCID mice. There was some improvement in islet survival and function after transplantation [107].

Adv vectors have several particular advantages for gene silencing in human islets. (1) Thorough understanding of the structure of Adv DNA may provide much flexibility in genetically engineer Adv vectors to make them more proper for shRNA delivery and expression. For example, it is convenient to construct shRNA expression vectors with different promoters and backbones with commercially available Adv shuttle vectors for optimal gene silencing; (2) high expression levels achieved by Adv vectors will help to increase gene silencing efficiency and help to get more beneficial effect. (3) Adv vectors has the capacity to carry a large insert, which will make it possible to express multiple shRNAs; or to co-express an shRNA to silence harmful genes and a cDNA to protect islets [117]. The disadvantage of Adv vector is the transient expression profile, which is not suitable for long-term gene silencing. Since the majority of islet cell death occurs within several days after transplantation, the use of Adv to silence genes involved in this process is appropriate.

\subsubsection{Lentivirus}

Lentivirus is a type of retroviral vector, which reverse transcribes into DNA, be actively transported into the nucleus, and integrate into the genome of target cells. Therefore, stable gene expression or silencing could be achieved by using lentiviral vector [252]. Unlike other retrovirus such as moloney murine leukemia virus, which could only transduce dividing cells, lentivirus can transduce both dividing and non-dividing cells. 
Vesicular stomatitis virus glycoprotein (VSV-G) pseudotyped lentiviral vectors is the most commonly used vector, which could transduce a broad range of cell types [253]. Lymphocytic choriomeningitis virus (LCMV)-pseudotyped is more efficient in transducing human islet insulin-secreting $B$ cells and shows less toxicity compared with VSV-G pseudotyped lentivirus [254]. Lentivirus were successfully used for genetic modification of islets with various genes, including reporter genes (GFP, Luciferase) [255, 256], antioxidative genes [257], antiapoptotic genes (cFLIP) [255], antiinflammatory and immunoregulatory genes (CTLA4, TGF- $\beta$, interleukin-1 receptor antagonist, and IL-4) [258-260]. Unlike Adv vectors which give high level transient gene expression, a prolonged gene expression is usually achieved after transduction islets of lentiviral vectors. For example, after transduction of islets with lentiviral vectors encoding a luciferase gene, bioluminescence is persistent for above 140 days post transplantation. However, the bioluminescence of islets transduced with Adv vector encoding a luciferase gene decrease from thousand fold at the beginning to ten fold over background at 60 days posttransplantation [256]. An optimized ex vivo transduction procedure should neither induce significant toxicity nor affect the insulin release function of islets [257]. In addition, lentiviral vectors-induced immune system activation was also minimal [260]. Under optimized conditions, lentivirus could transduce around $10 \%$ to $30 \%$ of islet cells in an intact islet, which are mainly the cells at the periphery [255, 261, 262]. This is still enough to have significant effect on the viability, probably due to the "barrier" effect, that is shield the core of islets by protecting the periphery of islets [255].

Several other approaches have also been investigated to improve the transduction efficiency. Callewaert et al. demonstrated increased transduction efficiency from around $11.2 \%$ to $80.0 \%$ by dissociation of islets to single cells prior to transduction and reaggregation of islets before transplantation [262]. However, improvement in the transfection efficiency did not translate into the improved function of islets. More cells are needed to achieve normoglycemia compared with intact islets. Barbu et al. tested the possibility to transduce islets by whole pancreas perfusion. This transduction procedure could transduce $30 \%$ of the cells, while keeping the structural integrity of islets. However, the cells within the core of islets still cannot be effectively transduced [263].

Lentiviral vector has also been used for shRNA delivery for gene silencing [264]. Because of the long term expression achieved by lentiviral vectors, it has been used to stably silence harmful genes to prevent the death of islet $\beta$ cells. For example, transduction of lentiviral vectors carrying shRNA against iNOS gene, significantly reduced IL-1 $\beta$-induced iNOS expression in islet $\beta$ cells and protect $\beta$ cells from IL-1 $\beta$ induced cell death [113]. Since the silencing effect achieved by lentiviral vector is long term, it is suitable for delivery of shRNA against genes that need to be continuously silenced. Otherwise, an inducible or switchable expression cassette should be used to control the timing of gene silencing and thus avoid potential side effects. 


\subsection{Concluding Remarks}

Theoretically, any gene of interest could be silenced or effectively inhibited, which is unachievable by conventional small molecule chemicals. In the past few years, RNAi technology has moved rapidly from bench to bedside at unprecedented speed. Currently, there are more than ten ongoing and completed clinical trials using siRNA as therapeutics (http://clinicaltrials.gov). It is interesting to note that one of these trials sponsored by Quark Pharmaceuticals was related to kidney transplantation, where a siRNA named I5NP was tested for the prophylaxis of delayed graft function in kidney transplantation patients. Although recent progresses have already demonstrated its great potential, the application of RNAi technology for improving the outcome of islet transplantation is still a fledgling area. Further understanding of the biological perspective of the islets transplantation is essential, as it may help to discover effective RNAi target genes. A combination of multiple gene silencing or simultaneously gene silencing and gene overexpression is likely to have improved beneficial effects through synergistic effects. Meanwhile, the advances in RNAi technology will enable us to design more potent siRNA or shRNAs, while minimizing the side effects caused by off-target effects and immune simulation. Last but not the least, the development of safe and efficient delivery systems is the key for successful gene silencing. 


\section{CHAPTER 3. INOS GENE SILENCING PREVENTS INFLAMMATORY CYTOKINE-INDUCED $\beta$ CELL APOPTOSIS*}

\subsection{Introduction}

Human islet transplantation is a promising therapeutic strategy for treating type I diabetes mellitus. Despite recent success, islet transplantation still lags behind primarily because a large number of transplanted islets do not function. This results in the need for multiple islet infusions for each patient. Most islet grafts are destroyed due to immune and inflammatory reaction mediated by proinflammatory cytokines, such as interleukin- $1 \beta$ (IL-1 $\beta$ ), tumor necrosis factor- $\alpha$ (TNF- $\alpha$ ), and interferon- $\gamma$ (IFN- $\gamma$ ). These proinflammatory cytokines induce a series of intracellular inflammatory signal cascades, leading to $\beta$-cell death, probably due to apoptosis, necrosis, and other processes [52]. The stimulation of inducible nitric oxide synthase (iNOS) expression and consequent production of radical nitric oxide (NO) as well as of the other radicals, such as peroxynitrite (OONO-) and superoxide (O2-) represent a pivotal step in these processes $[48,63,265,266]$. The inhibition of iNOS has shown significant protection of $\beta$ cells from proinflammatory cytokine-induced damage [113, 267, 268]. Therefore, it is reasonable to suggest that iNOS might be a potential target for improving the outcome of human islet transplantation.

RNA interference (RNAi) is a promising therapeutic strategy which utilizes an evolutionary conserved biologic process to induce sequence-specific, post-transcriptional gene silencing by small interference RNA (siRNA) [269]. siRNA-mediated gene silencing in rodent $\beta$-cells and pancreatic islets has been shown to inhibit a target gene [270, 271]. In mouse insulinoma cells, Burkhardt et al. [108] demonstrated that siRNA targeting Fas, another important mediator of $\beta$-cell death, was able to silence Fas gene expression and inhibit Fas-mediated $\beta$-cell damage in response to inflammatory cytokines. Keeping these promising findings in mind, we recently tested the effect of chemically synthesized siRNAs against iNOS and NF- $\kappa \mathrm{B}$ on gene expression, NO production, and apoptosis in insulin producing $\beta$-cell line (INS-1E) and demonstrated that silencing of iNOS gene to be more effective than that of NF- $\kappa B$ [116]. There are also conflicting reports on the role of NF- $\mathrm{BB}$ on pancreatic $\beta$-cell death $[62,272,273]$. McCabe and O'Brien used lentiviral vector-based shRNA delivery to suppress IL-1 $\beta$ mediated induction of iNOS expression, resulting in significant protection against the cytotoxic effects of IL-1 $\beta$ exposure $[113,114]$. However, these authors used rat insulinoma (RIN-r) cell lines and not human islets. Moreover, they did not determine the functional viability of RIN-r cells by measuring glucose-stimulated insulin release. Taking care of the usefulness of iNOS gene silencing in islet transplantation, in this study, we compared transfection efficiency and effect of iNOS gene silencing on

* Adapted with permission. F. Li, R.I. Mahato, iNOS gene silencing prevents inflammatory cytokine-induced $\beta$ cell apoptosis, Mol Pharm, 5 (2008) 407-417. 
apoptosis in INS-1E cells and intact human islet, which is a cluster of 200-1000 cells and hard to transfect.

This report implicated the pivotal role of iNOS in cytokine-induced $\beta$ cell death. We demonstrate the possibility of using iNOS gene silencing as a strategy to improve the outcome of human islet transplantation.

\subsection{Materials and Methods}

\subsubsection{Materials}

Fetal bovine serum (FBS), sodium pyruvate, and CMRL 1066 medium were purchased from Mediatech, Inc (Herndon, VA). Penicillin/streptomycin, phosphate-buffered saline (PBS), $0.25 \%(\mathrm{w} / \mathrm{v})$ trypsin-EDTA and RPMI-1640 medium and OptiMEM-I reduced serum medium were purchased from GIBCO-BRL (Gaithersburg, MD). 2-

Mercaptoethanol was obtained from Sigma Aldrich (St. Louis, MO). Recombinant IL-1 $\beta$, TNF- $\alpha$ and IFN- $\gamma$ were purchased from R\&D Systems (Minneapolis, MN). siRNA against rat iNOS (Gene Bank \# NM_012611, Table 3.1) and human iNOS (Gene Bank \# NM_000625, Table 3.2), control siRNA, fluorescein-labeled siRNA, and Lipofectamine 2000 were purchased from Invitrogen (Carlsbad, CA). All primers used for real time RTPCR were obtained from Integrated DNA Technology (Coralville, IA). TUNEL assay kit was from BD Pharmingen (Franklin Lakes, NJ). Caspase 3 cellular activity assay kit was purchased from EMD Biosciences, Inc. (San Diego, CA). RNeasy Mini kit and RNaseFree DNase Set were purchased from Qiagen (Valencia, CA). Multiscribe reverse transcription kit was from Applied Biosystems (Foster City, CA). SYBR Green-I dye universal PCR master mix and other reagents for real time RT PCR were from Roche (Indianapolis, IN). Griess assay kit was from Promega (Madison, WI). BCA protein assay kit was from Pierce Chemical Company (Rockford, IL).

\subsubsection{Cell culture and transfection}

Rat insulin producing $\beta$-cells (INS-1E cells), a kind gift from Professor Claes B. Wolheim (University Medical Center, Geneva, Switzerland), were cultured at $37^{\circ} \mathrm{C}$ in a humidified atmosphere containing 5\% CO2 in complete medium composed of RPMI 1640 supplemented with $10 \%$ fetal bovine serum (FBS), $1 \mathrm{mM}$ sodium pyruvate, $50 \mu \mathrm{M}$ 2-mercaptoethanol, $100 \mathrm{U} / \mathrm{mL}$ penicillin, and $100 \mu \mathrm{g} / \mathrm{mL}$ streptomycin.

INS-1E cells were seeded $24 \mathrm{~h}$ before transfection in a 24 -well plate $\left(5 \times 10^{5}\right.$ cells/well $)$ for all the experiments except for TUNEL assay, for which we used 12-well plates $\left(10^{6}\right.$ cells/well). siRNAs were transfected into INS-1E cells after complex formation with Lipofectamine 2000 according to the manufacturer's instructions. Briefly, siRNA molecules and Lipofectamine 2000 were mixed together in OPTMEM I reduced serum medium at a ratio of $30 \mathrm{nmol} / 1 \mathrm{mg}$ (siRNA/ Lipofectamine 2000), and complex 
Table 3.1 Small interfering RNA (siRNA) sequences of rat iNOS (NM_012611).

\begin{tabular}{|c|c|c|}
\hline Start site & siRNA sequence & Coding region* \\
\hline 224 & $\begin{array}{l}\mathrm{S}_{\dagger}+\text { 5'-CUACCAAGGUGACCUGAAAGA[dT][dT]-3' }^{\prime} \\
\text { AS }+ \text { : 5'-UCUUUCAGGUCACCUUGGUAG[dT][dT]-3' }\end{array}$ & $224-244$ \\
\hline 675 & $\begin{array}{l}\text { S: 5'-GAAGCCGUAACAAAGGAAAUA[dT][dT]-3', } \\
\text { AS: 5'-UAUUUCCUUUGUUACGGCUUC[dT][dT]-3' }\end{array}$ & $675-695$ \\
\hline 898 & $\begin{array}{l}\text { S: 5'-CCUCGGAUAUCUCUUGCAA[dT][dT] -3' } \\
\text { AS: 5'-UUGCAAGAGAUAUCCGAGG[dT][dT] -3' }\end{array}$ & $898-916$ \\
\hline 2225 & $\begin{array}{l}\text { S:5'-GUUCGAUGUUCGAAGCAAACA[dT][dT] -3' } \\
\text { AS: 5'-UGUUUGCUUCGAACAUCGAAC[dT][dT] -3' }\end{array}$ & $2225-2245$ \\
\hline
\end{tabular}

$*$ ORF region; $\uparrow \mathrm{S}$, sense; $\ddagger \mathrm{AS}$, antisense. 
Table 3.2 Small interfering RNA (siRNA) sequences of human iNOS (NM_000625).

\begin{tabular}{clc}
\hline \multirow{2}{*}{ Start site } & \multicolumn{1}{c}{ siRNA sequence } & Coding region* \\
\hline \multirow{2}{*}{511} & S $\uparrow:$ 5'-CGGCAUGUGAGGAUCAAAA[dT][dT] -3' & \\
& AS + 5'-UUUUGAUCCUCACAUGCCG[dT][dG] -3' & $511-529$ \\
& S: 5'-GCGGGAUGACUUUCCAAGA [dT][dT] -3' & \\
539 & AS: 5'-UCUUGGAAAGUCAUCCCGC[dT][dG] -3' & $539-557$ \\
& S:5'-GCUAUCGAAUUUGUCAACC [dT][dT] -3' & \\
691 & AS: 5'-GGUUGACAAAUUCGAUAG [dT][dT] -3' & $691-709$ \\
\hline
\end{tabular}

$*$ ORF region; $\uparrow \mathrm{S}$, sense; $\$ \mathrm{AS}$, antisense. 
formation was allowed to proceed for $30 \mathrm{~min}$ at room temperature before adding into INS-1E cells. At $12 \mathrm{~h}$ post transfection, medium containing lipid/siRNA complexes were replaced with fresh medium.

To determine the transfection efficiency and effect of iNOS gene silencing on protection of human islets from proinflammatory cytokines, we re-designed three siRNA sequences targeting different regions of human iNOS mRNA (Table 3.2), and used for transfection into human islets. These islets were received from one of the several Islet Cell Resource (ICR) Centers through ICR Services for Basic Science Applications. On arrival at our facility, islets were cultured at $37^{\circ} \mathrm{C}$ in CMRL 1066 medium supplemented with $10 \%$ FBS. Human islets were seeded at 24 -well plate (2000 IE/well) 2 hours before transfection and were transfected with siRNA after complex formation with Lipofectamine 2000 as described above.

\subsubsection{Fluorescence microscopy and flow cytometry}

To determine the transfection efficiency, INS-1E cells and human islets were transfected with fluorescein-labeled siRNA after complex formation with Lipofectamine 2000. At 12 $\mathrm{h}$ post transfection, cells were visualized under fluorescence microscopy. To determine the percentage of transfected cells, INS-1E cells were washed with 1 x PBS, trypsinized, and suspended in $1 \times$ PBS. Since human islets are a cluster of cells, they were treated with trypsin-EDTA for $10 \mathrm{~min}$ at $37^{\circ} \mathrm{C}$ followed by vigorous pipeting to create a singlecell suspension. Then the cell suspension were directly introduced to a FACSCalibur ${ }^{\mathrm{TM}}$ Flow Cytometer (Becton Dickinson, NJ) equipped with a $488 \mathrm{~nm}$ argon ion laser. The FL-1 emission channel was used to monitor the fluorescein and results from 10,000 fluorescent events were obtained for analysis. Cells without transfection were served as negative controls.

\subsubsection{Real time RT-PCR}

Expression of rat iNOS gene in INS-1E cells and that of human iNOS in human islets was determined at mRNA level by real time RT-PCR. Following treatment, total RNA was extracted with RNeasy Mini KIT and treated with DNase by on-column digestion (RNase-Free DNase Set). RNA concentration was determined by spectrophotometer (NanoDrop). One hundred and seventy nanograms total RNA was converted into cDNA using multiscribe reverse transcriptase reagents and random hexamers at a $10 \mu 1$ reaction system. Two microliters of cDNA were used as a template and analyzed by SYBR Green-I dye universal PCR master mix on LightCycler 480 Instrument. The primers used for real-time PCR were as follows: rat iNOS [116] Forward: 5'-

ACCAGAGGACCCAGAGACAAGC-3'; Reverse: 5'TCCAGGCCATCTTGGTGGCAAA-3' (amplicon size 208 bp); rat 18S rRNA [274]:Forward: 5'-CGG CTA CCA CAT CCA AGG AA-3' ; Reverse : 5'-GCT GGA ATT ACC GCG GCT-3'(amplicon size 186 bp); human iNOS [275]: Forward 5'ACGTGCGTTACTCCACCAACA-3'; Reverse 5'-CATAGCGGATGAGCTGAGCA- 
3'(amplicon size 102 bp); human ribosomal protein S19 (human S19); Forward 5' GCTTGCTCCCTACCGATGAGA-3; Reverse 5'-ACCCCGGAGGTACAGGTG3'(amplicon size $73 \mathrm{bp}$ ). To assess the specificity of the amplified PCR product, the Melting Curve Analysis was performed on the LightCycler 480 Instrument. The results iNOS mRNA level were compared by calculating the $\mathrm{CP}$ value and normalized by the reference genes (rat $18 \mathrm{~S}$ or human S19). Four independent experiments were performed and the results were expressed as a percentage of control.

\subsubsection{Griess assay}

Nitric oxide (NO) is rapidly oxidized in culture medium into nitrite, which accumulate in the sample and can be easily correlated with NO production. Therefore, nitrite concentration was determined using the Griess assay (Promega, Madison, MI). Fifty microliters of cell culture supernatant was added to a 96 well-plate and mixed with $50 \mu \mathrm{L}$ of $1 \%$ sulfanilamide in 5\% phosphoric acid solution and incubated for $5 \mathrm{~min}$ at room temperature in dark. Then, $50 \mu \mathrm{L}$ of $0.1 \% \mathrm{~N}-1$-napthylethylenediamine dihydrochloride (NED) aqueous solution was added to each well. The plate was incubated for additional $10 \mathrm{~min}$, and absorbance was measured at $560 \mathrm{~nm}$ using a microplate reader. To determine the nitrite concentration in each sample, a standard curve was prepared using nitrite standard solution and culture medium as matrix.

\subsubsection{Effect of iNOS gene silencing on apoptotic cell death}

\subsubsection{Microscopy}

To determine whether iNOS gene silencing inhibits apoptosis of $\beta$ cells due to inflammatory cytokines, INS-1E cells $\left(10^{6}\right.$ cells/well in a 12 well plate) were transfected with siRNA against iNOS as well as with control siRNA. The cells were incubated with a cocktail of TNF- $\alpha(50 \mathrm{pg} / \mathrm{mL}), \mathrm{IL}-1 \beta(5 \mathrm{ng} / \mathrm{mL})$ and IFN- $\gamma(50 \mathrm{ng} / \mathrm{mL})$ for $48 \mathrm{~h}$, then visualized under microscopy.

\subsubsection{Caspase 3 activity assay}

The cellular caspase 3 activity of INS-1E cells or human islets was measured with Caspase 3 Cellular Activity Assay Kit. INS-1E cells were seeded at a density of $5 \times 10^{5}$ cells/well in 24-well plate; human islets were seeded at a density of $2000 \mathrm{IE} /$ well in 24well plate. After treatment, cells were collected and lysed with lysis buffer. Ten microliters of the cell lysate solution was added in $40 \mu \mathrm{L}$ assay buffer and mixed with 50 $\mu \mathrm{L}$ caspase 3 substrate (Ac-DEVD-pNA) at a half volume 96-well plate. The mixture was kept at $37^{\circ} \mathrm{C}$ and absorbance at $405 \mathrm{~nm}$ was read continuously using a spectrophotometer every $5 \mathrm{~min}$ for one hour. The absorbance versus time for each sample was plotted and the activity was calculated from the slope of the linear curve. To normalize the activity, total protein concentration of each sample was also measured with $\mathrm{BCA}$ protein assay kit. 


\subsubsection{TUNEL assay}

To gain insights on the mechanism of $\beta$ cell death, a terminal deoxynucleotidyltransferase dUTP nick end labeling (TUNEL) assay with the APODIRECTTM kit was used to detect apoptotic cells. Following transfection and incubation with a cytokine cocktail, INS-1E cells and human islets were made to single cell suspension as described above. The cells were fixed in 1\% paraformaldehyde in PBS $(\mathrm{pH}$ 7.4) and then treated with ice-cold 70\% ethanol, stained with FITC-dUTP, and then with propidium iodide. The intensity of fluorescence was measured by a flow cytometer and analyzed using CELLQUEST software (BD Bioscience). Non-transfected cells without cytokine treatment were served as a negative control. Three sets of independent transduction experiments were carried out for each assay.

\subsubsection{Assessment of human islet function post-transfection}

The ability of human islets to respond to glucose stimuli was used to determine the effect of transfection of Lipofectamine 2000/siRNA complexes on human islet function. Following transfection, islets were incubated with a cocktail of inflammatory cytokines including TNF- $\alpha(50 \mathrm{pg} / \mathrm{mL}), \mathrm{IL}-1 \beta(5 \mathrm{ng} / \mathrm{mL})$ and IFN- $\gamma(50 \mathrm{ng} / \mathrm{mL})$ for $48 \mathrm{~h}$. Then, islets were challenged with two different concentration of glucose $(60 \mathrm{mg} / \mathrm{dL}$ and 300 $\mathrm{mg} / \mathrm{dL}$ ) for $1 \mathrm{~h}$ at $37^{\circ} \mathrm{C}$. Supernatants were then collected for measuring insulin secretion by human insulin ELISA Kit (Alpco Diagnostics, Windham, NH). Results were normalized by measuring the total protein using a BCA assay kit. Stimulation index was calculated by measuring insulin release by islets $1 \mathrm{~h}$ after incubation with media containing basal $(60 \mathrm{mg} / \mathrm{dL})$ and stimulated $(360 \mathrm{mg} / \mathrm{dL})$ levels of glucose.

\subsubsection{Statistical analysis}

The difference between groups was determined by unpaired t-test and a $\mathrm{P}<0.05$ was considered statistically significant. Results are expressed as the means \pm standard deviation (SD).

\subsection{Results}

\subsubsection{Transfection efficiency of lipid/siRNA complexes in rat $\beta$ cells}

We used fluorescein labeled siRNA at a concentration of 50,100, and $150 \mathrm{nM}$ to quantitatively asses the incorporation of siRNA into rat $\beta$ cells. After $12 \mathrm{~h}$ of incubation with Lipofectamine 2000/fluorescein-labelled siRNA complexes, cells were observed under fluorescence microscopy. Figure 3.1A demonstrates that these cells were effectively transfected with fluorescein-labeled siRNA. The photographs show green cells with distinct spots speckled their surface and in the nucleus, suggesting enhanced 
A
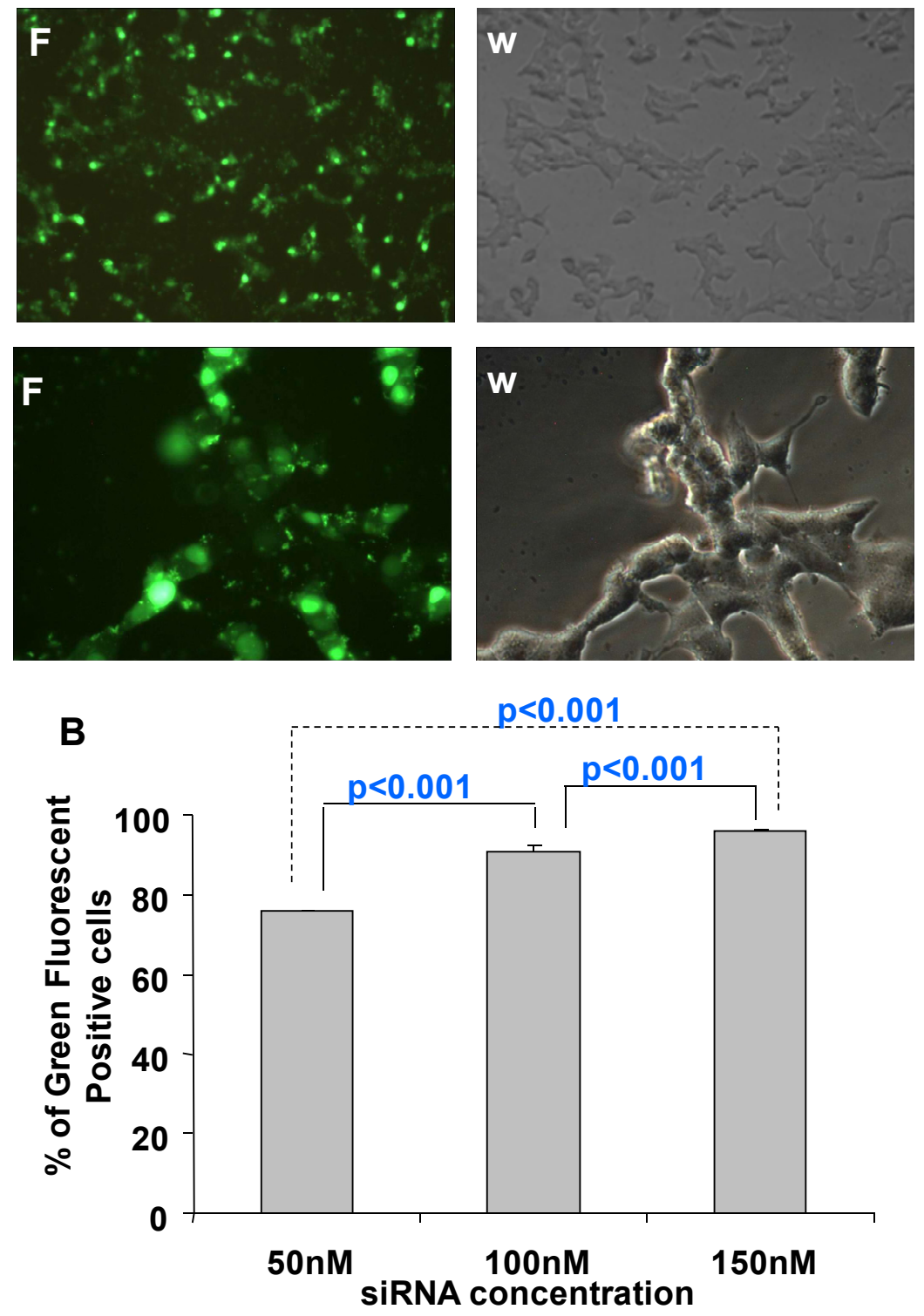

Figure 3.1 Transfection efficiency of siRNA into rat insulin producing $\beta$ cells.

INS-1E cells were transfected with fluorescein-labeled siRNA, after complex formation with Lipofectamine 2000 at siRNA concentration of 50,100,150 nM. At $12 \mathrm{~h}$ after transfection, cells were visualized under fluorescence microcopy and analyzed with flow cytometry. (A) Fluorescent images showing fluorescein-labeled siRNA incorporation into INS-1E cells. Left panels show cells under visible light, and right panel under visible light. Upper panel is low magnification $(100 \mathrm{X})$ and lower panel is high magnification $(400 \mathrm{X})$. (B) Percentage of green fluorescence positive cells. Results are the mean $\pm \mathrm{SD}$, $n=3$. The $p$ value of $<0.05$ was considered statistically significant. 
cellular uptake and nuclear translocation. To quantitatively determine the transfection efficiency, cells were analyzed by flow cytometry. As shown in Figure 3.1B, the transfection efficiency increased from $75.9 \%$ to $95.9 \%$ with increase in siRNA concentration from $50 \mathrm{nM}$ to $150 \mathrm{nM}$. The average fluorescence intensity also increased accordingly from $2.48 \times 10^{4}$ to $6.72 \times 10^{4}$ (Data not shown).

\subsubsection{Effect of siRNA sequence on iNOS gene silencing and NO production on rat $\beta$ cells}

Figure 3.2A demonstrates the effect of siRNA sequences on iNOS gene silencing. All four siRNA sequences were able to silence iNOS expression and siRNA-iNOS-898 showed silencing around $50 \%$ of iNOS expression. Figure 3.2B shows the effect of siRNA sequence on the NO production by rat $\beta$ cells. Compared with control siRNA, all four siRNAs targeting different regions of rat iNOS mRNA showed inhibition of NO production by rat $\beta$ cells. Among them siRNA-iNOS- 898 was the most potent one, which reduced the NO production by $60 \%$. Therefore, siRNA 898 was used for further studies on rat $\beta$ cells. We also noticed that there was almost no change in the NO production in the control siRNA treated group (Figure 3.2B), which indicates the inhibition of NO production was due to the specific silencing of iNOS mRNA, and not due to the nonspecific effects.

\subsubsection{Effect of iNOS gene silencing on rat $\beta$-cell death}

To determine whether iNOS gene silencing inhibit apoptosis of rat $\beta$-cells induced by inflammatory cytokines, INS-1E cells $\left(10^{6} /\right.$ well in a 12 well plate) were transfected with siRNA against rat iNOS as well as the control siRNA. Then the cells were incubated with cytokine cocktail (IL-1 $\beta 50 \mathrm{pg} / \mathrm{mL}, \mathrm{TNF}-\alpha 5 \mathrm{ng} / \mathrm{mL}$, and IFN- $\gamma 50 \mathrm{ng} / \mathrm{mL}$ ) for $48 \mathrm{~h}$. As visualized under microscopy, almost all the cells transfected with the control siRNA were aggregated and round up, indicating cell death, probably due to apoptosis (Figure 3.3A). In contrast, only a small part of the cell treated with siRNA-iNOS were aggregated, indicating only a small fraction of the cells were undergoing apoptosis.

One of the later steps in apoptosis is DNA fragmentation, which results from the activation of endonucleases during the apoptotic process. Therefore, the analysis of DNA fragmentation with TUNEL assay is a useful approach to get information regarding cell apoptosis. As shown in Figure 3.3B, cells with no cytokine treatment showed $0.8 \%$ apoptotic cells, cells transfected with control siRNA treated with cytokines showed $42.3 \%$ apoptotic cells. The cells transfected with siRNA against iNOS and treated with cytokines showed $12.3 \%$ apoptotic cells; clearly demonstrating that iNOS gene silencing can help protect $\beta$ cell from proinflammatory cytokines. 

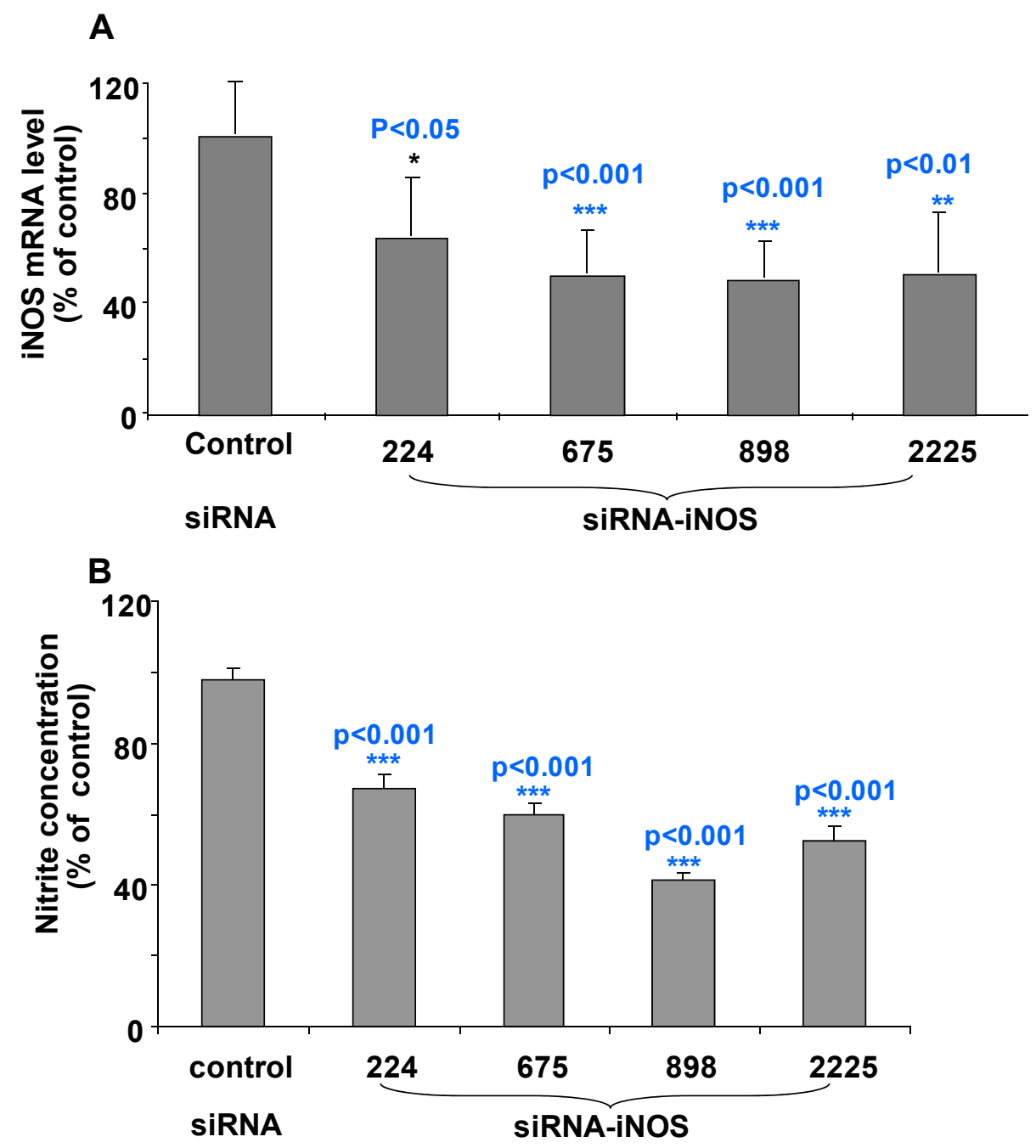

\section{Figure 3.2 Effect of siRNA sequences on iNOS gene expression and NO production in rat $\beta$ cells.}

Effect of siRNA sequences on iNOS gene expression, (A) and NO production (B) in rat $\beta$ cells. Following transfection of INS-1E cells with $150 \mathrm{nM}$ siRNA-iNOS complexed with $5 \mu \mathrm{g} / \mathrm{mL}$ Lipofectamine 2000, cells were treated for additional $12 \mathrm{~h}$ with the cytokine cocktail of IL-1 $\beta(50 \mathrm{pg} / \mathrm{mL})$, TNF- $\alpha(5 \mathrm{ng} / \mathrm{mL})$, and IFN- $\gamma(50 \mathrm{ng} / \mathrm{mL})$. Cells not treated with the cytokines were used as negative control. iNOS expression is expressed as the amount of iNOS mRNA measured by real time RT-PCR. Nitrite concentration is expressed as percentage of positive control. Results are the mean $\pm \mathrm{SD}(\mathrm{n}=3)$ and were normalized with live cells. The $\mathrm{p}$ value of $<0.05$ was considered statistically significant. 
A
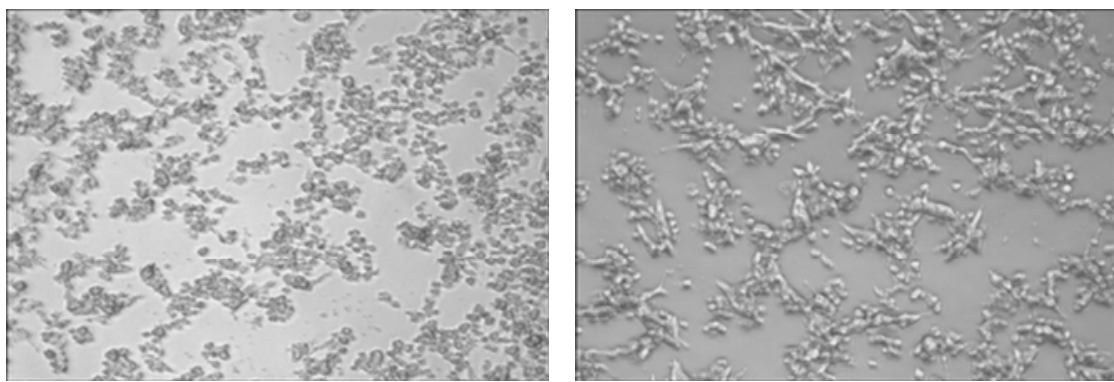

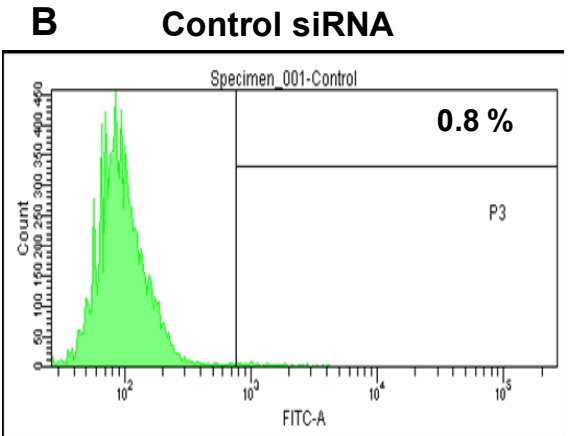

negative control

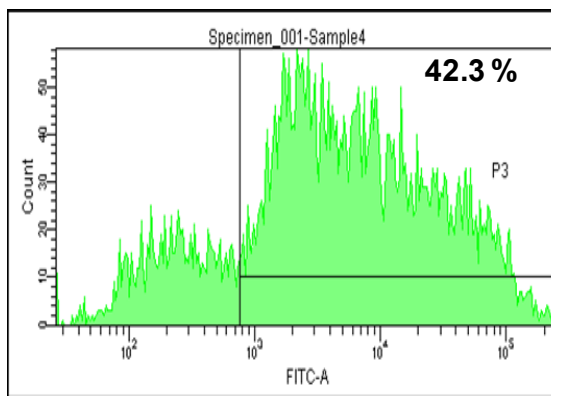

control siRNA
SIRNA-iNOS

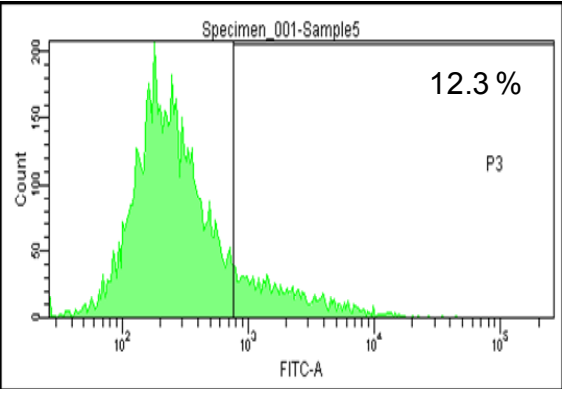

SiRNA-iNOS

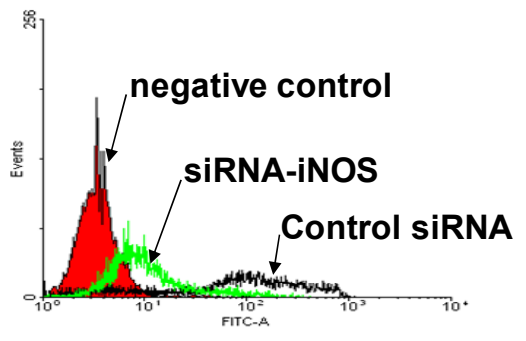

Figure 3.3 Effect of iNOS gene silencing on rat $\beta$ cell death.

Following transfection of INS-1E cells with siRNA-iNOS-898 as well as the control siRNA, the cells were incubated with a cytokine cocktail of IL- $\beta(50 \mathrm{pg} / \mathrm{mL}), \mathrm{TNF}-\alpha(5$ $\mathrm{ng} / \mathrm{mL}$ ), and IFN- $\gamma(50 \mathrm{ng} / \mathrm{mL})$ for $48 \mathrm{~h}$, then visualized under microscopy (A) and analyzed by TUNEL assay (B). A), left panel, most cells treated with the control siRNA were aggregated and round up, indicating cell death; right panel, only a part of the cell treated with siRNA-iNOS were aggregated and indicating only a small fraction of the cells were undergoing apoptosis. B), TUNEL assay of INS-1E cells treated with siRNAiNOS-898 as well as control siRNA. Negative control, cells with no cytokine treatment; siRNA-iNOS, cells treated with siRNA-iNOS-898 and cytokine; control siRNA, cells treated with control siRNA and cytokine. The values show the percentage of positive cells. 


\subsubsection{Transfection efficiency of lipid/siRNA complexes into human islets}

After confirming the beneficial effect of iNOS gene silencing on rat $\beta$-cells from proinflammatory cytokines, we used fluorescein-labeled siRNA at doses of 100 and 400 $\mathrm{nM}$ to determine the transfection efficiency in human islets under fluorescence microscopy. Figure 3.4A demonstrates that human islets were transfected with fluorescein-labeled siRNA. While the untreated control human islets showed only weak background fluorescence, human islets transfected with fluorescein-labeled siRNA showed distinct spots speckled throughout their surface with some concentrations noted at the edges and over their interior surface. These images clearly demonstrated that siRNA was introduced into human islets in culture, but we could not determine whether siRNA has penetrated into the interiors of human islets. Thus, transfected human islets were analyzed by flow cytometry upon dispersion into single cells after treating with trypsin-EDTA for $10 \mathrm{~min}$ at $37^{\circ} \mathrm{C}$ followed vigorous pipeting. Both the fluorescent images for siRNA dose titration and the more quantitative FACS date help document how much siRNA was incorporated into islet cells. Almost $21.5 \%$ and $28.3 \%$ islet cells incorporated fluorescein-labeled siRNA when transfected with siRNA at a concentration of $100 \mathrm{nM}$ and $400 \mathrm{nM}$, respectively (Figure 3.4B).

\subsubsection{Effect of siRNA sequences on iNOS gene silencing in human islets}

Following screening of the siRNA sequences using INS-1E cells and demonstrating the beneficial effect of iNOS gene silencing on the protection of $\beta$-cells from inflammatory cytokines, we determined the effect of iNOS gene silencing in human islets. Since there is not $100 \%$ homogeneity in rat iNOS mRNA (Gene Bank Accession\# NM_012611) and human iNOS mRNA (Gene Bank Accession\# NM_000625), we re-designed three siRNA sequences targeting different regions of human iNOS and used for transfection into human islets. Quantitative real-time RT-PCR was used to measure the human iNOS gene expression level using SYBR green chemistry with human iNOS specific primer and human S19 as a reference gene. Figure 3.5A shows that all three siRNAs tested were able to reduce iNOS gene expression levels in human islets. Among all the siRNAs tested, siRNA-iNOS-691 was the most potent one, which reduced iNOS expression level by $50 \%$. Therefore, siRNA-iNOS-691 was used for further evaluation. Compared to the control siRNA group, there was significant decrease in NO production when human islets were transfected with lipid/siRNA-iNOS-691 complexes (Figure 3.5B). This result indicates that the inhibition of NO production was due to the specific silencing of iNOS mRNA and not due to the off-target effects.

\subsubsection{Effect of iNOS gene silencing on human islet cell death}

We have shown that siRNA against iNOS mRNA could reduce the apoptosis of rat $\beta$ cells. In addition, siRNA can be delivered into human islets and reduce iNOS mRNA level. Therefore, we continued to test whether iNOS gene silencing can also prevent the cytokine-induced apoptosis of human islets. TUNEL assay was used to detect DNA 
A

Visible
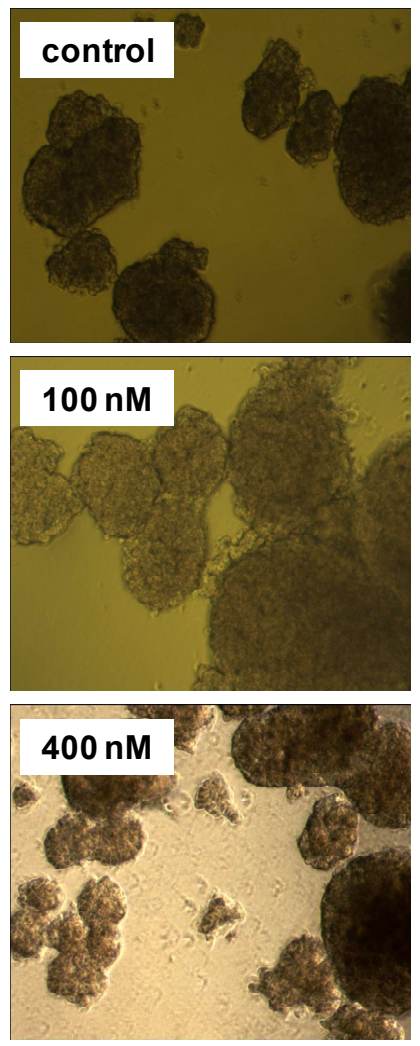

Fluorescence
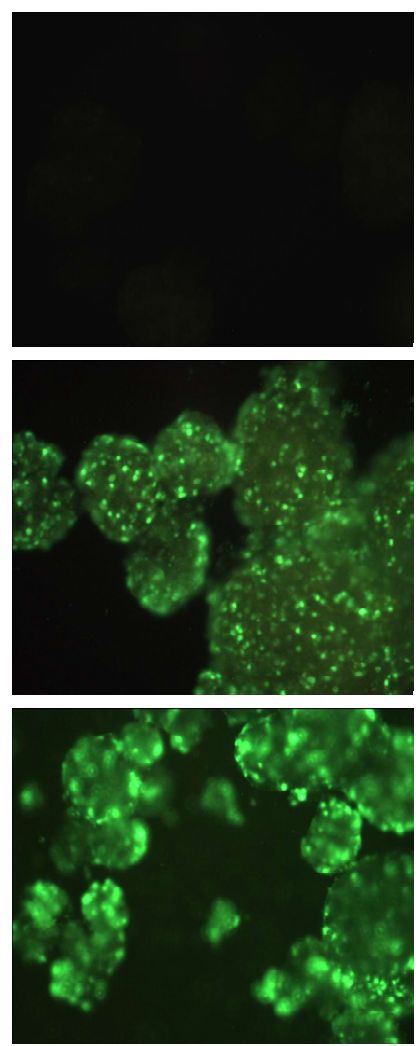

B
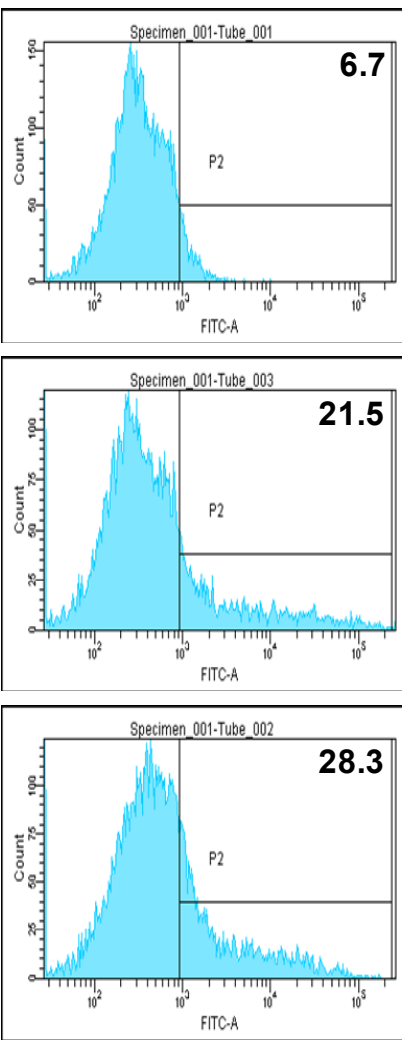

\section{Figure 3.4 Transfection efficiency of siRNA into intact human islets.}

Fluorescein-labeled siRNA were transfected into human islets and visualized under fluorescence microscope at $12 \mathrm{~h}$ post transfection. (A) Fluorescent images showing fluorescein-labeled siRNA incorporation into intact human islets, Left panels show human islets under visible light, and right panel under fluorescence. (B) flow cytometry analysis showing percentage of green fluorescence positive cells. 


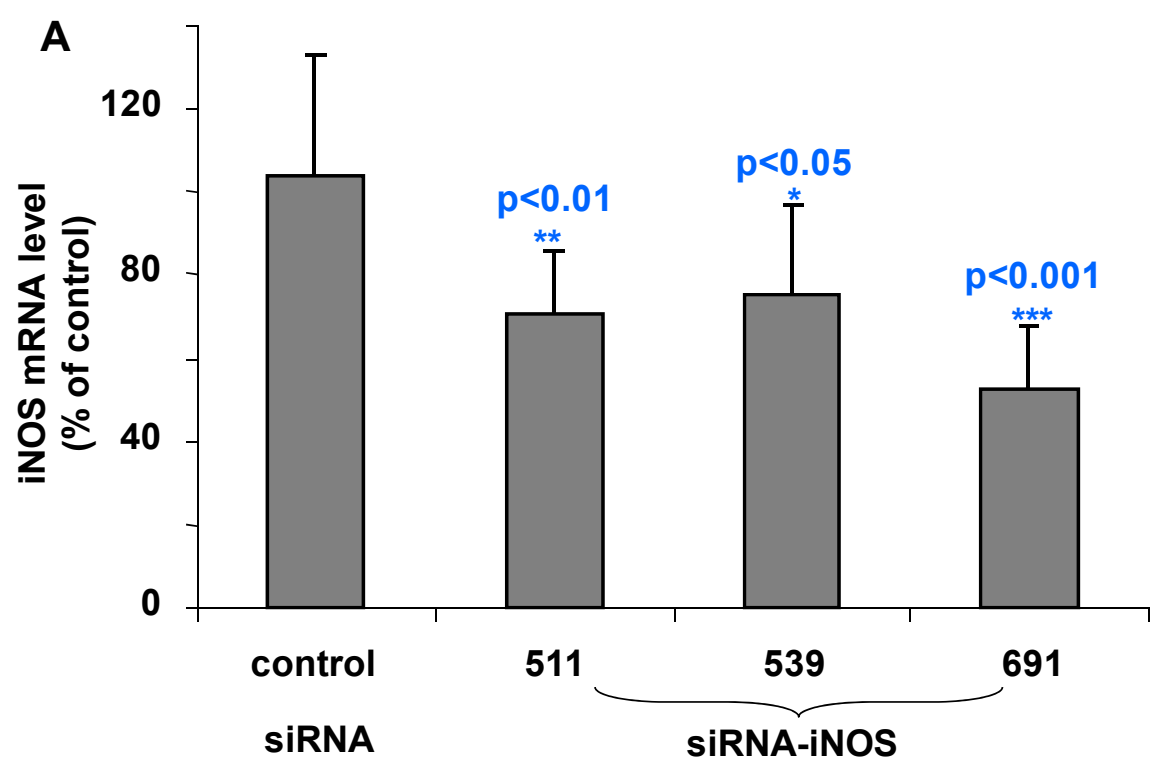

B

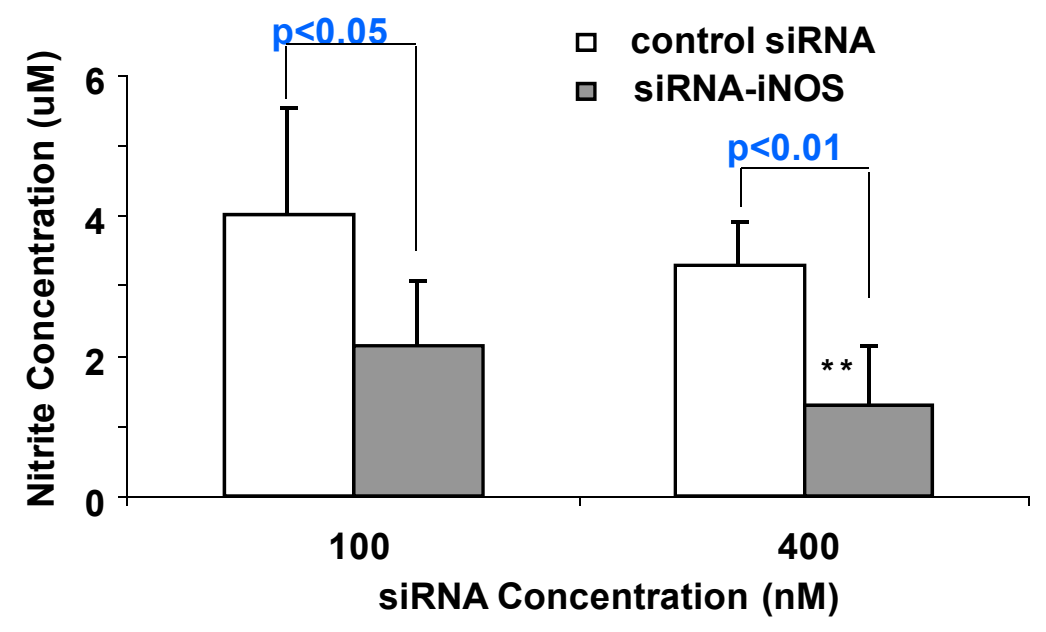

Figure 3.5 Effect of siRNA sequence on iNOS expression on human islets.

Human islets (2000 IE) were transfected for $12 \mathrm{~h}$ with $400 \mathrm{nM}$ iNOS-siRNA complexed with $13.3 \mu \mathrm{g} / \mathrm{mL}$ Lipofectamine 2000. Then human islets were treated for additional $12 \mathrm{~h}$ with a cytokine cocktail of IL- $1 \beta(50 \mathrm{pg} / \mathrm{mL})$, TNF- $\alpha(5 \mathrm{ng} / \mathrm{mL})$, and IFN- $\gamma(50 \mathrm{ng} / \mathrm{mL})$. The iNOS mRNA were measured by real time RT-PCR and expressed as the percentage of control. B) Nitrite concentration of the culture supernatants was determined by Griess assay. Human islets treated with control siRNA were used as control. Results are represented as the mean $\pm \mathrm{SD}, \mathrm{n}=3$. Significance established at $\mathrm{P}<0.05(*), \mathrm{P}<0.01$ $(* *)$, and $\mathrm{P}<0.001(* * *)$. 
fragmentation. As shown in Figure 3.6A, siRNA against human iNOS reduced the percentage of apoptotic cells to $28.5 \%$ compared to the control siRNA group (36.2\%). Although the prevention of apoptosis in human islets was not as high as shown in rat $\beta$ cells (Figure 3.3B and Figure 3.6A), this is not a surprise to get different results from INS-1E cell and human islets, which is a cluster of 200-1000 cells. Increased caspase 3 activity is often used as a marker for apoptotic signaling cascade [276]. Therefore, we tested the effects of iNOS gene silencing on caspase 3 activities of human islets (Figure 3.6B). Compared with the control siRNA treated group, siRNA-iNOS reduced human islets caspase 3 activity by around $25 \%$.

\subsubsection{Human islet function after iNOS gene silencing}

The effect of transfection process on in vitro islet function was determined by static incubation of islets with different concentration of glucose. There was increase in insulin secretion as a function of glucose concentration for islets transfected with lipid/siRNAiNOS complexes, with stimulation index of 1.40. In contrast, there was little increase in insulin secretion with increase in glucose concentration for islets transfected with lipid/siRNA-control (Figure 3.7). The results suggest that iNOS gene silencing partly protected human islet from inflammatory cytokine mediated destruction.

\subsection{Discussion}

Human islet transplantation has great potential for treating insulin dependent diabetes, allowing stable glucose homeostasis without exogenous insulin regimens and thus avoiding several diabetic glucose complications. Despite some recent improvements in transplantation technology, a large number of transplanted islets do not function properly, which results in the need for multiple islet infusions for each patient. Moreover, the success of islet transplantation is currently dependent on chronic treatment of the patient with strong immunosuppressant drugs, which are often associated with serious side effects. Due to the limited supply of cadaver donor, it is essential to improve the survival of transplanted islets. Therefore, we and others have been working on genetic modification of islets to improve the outcome of islet transplantation [12, 17, 64, 246, $247,277]$. The over-expression of therapeutics genes has been demonstrated to improve the survival and function of islets post-transplantation [246, 278]. Our previous work showed improved islet transplantation by genetic modification of human islets with human vascular endothelial growth factor (hVEGF) and human interleukin-1 receptor antagonist (hIL-1Ra) [246]. The expression of hVEGF and hIL-1Ra synergistically suppress islet dysfunction and NO production in human islets. After transplantation of these transfected human islets into diabetic mice, reduced blood glucose levels and increased blood insulin and c-peptide level was observed [246].

In contrast to the over-expression of therapeutic genes, ex vivo silencing of harmful genes, offers an alternative approach to induce human islets to modify their internal signal transduction pathways to prevent the damage inflicted upon them by 


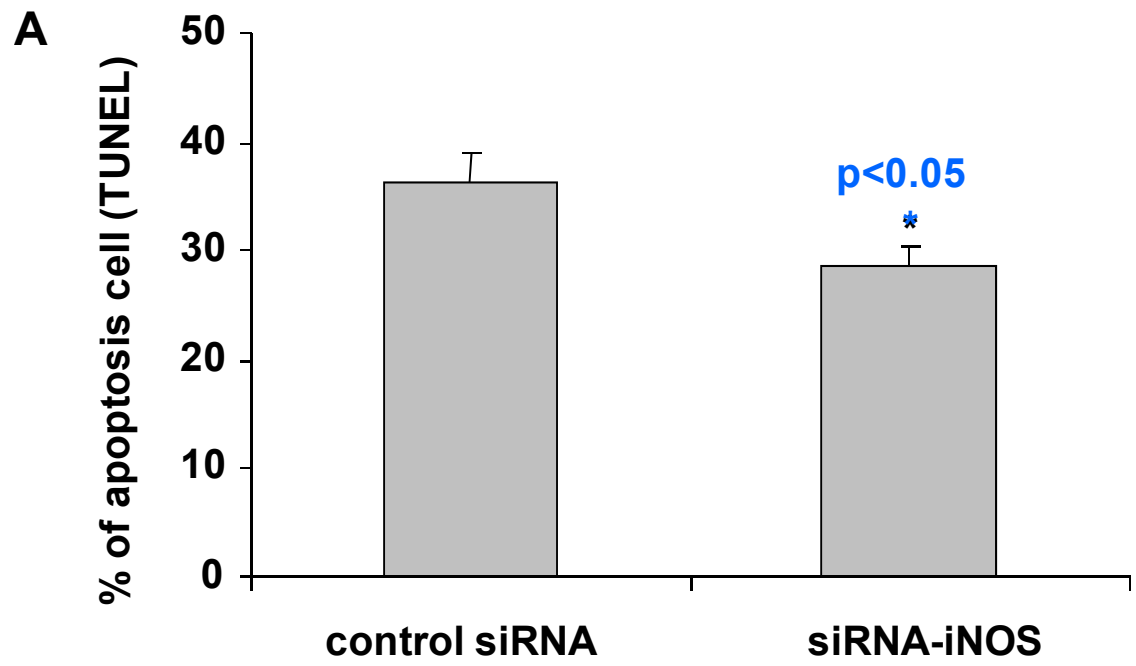

B

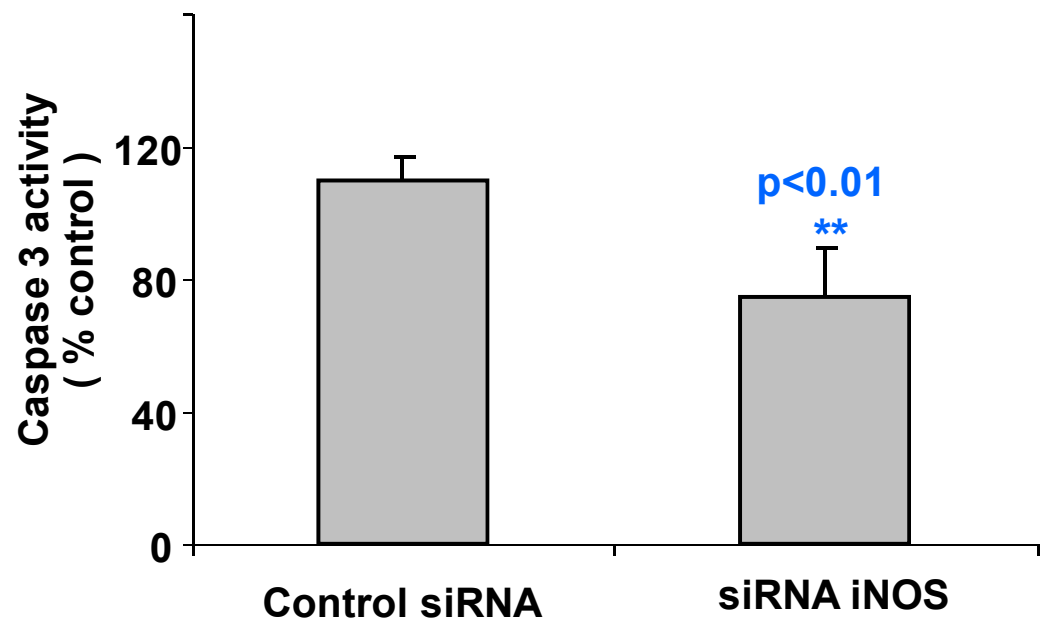

Figure 3.6 Effect of iNOS gene silencing on human islets cell death.

Following transfection with $400 \mathrm{nM}$ siRNA-iNOS-691 as well as the control siRNA, human islets (2000 IE) were incubated with the cytokine cocktail of IL-1 $\beta(50 \mathrm{pg} / \mathrm{mL})$, TNF- $\alpha(5 \mathrm{ng} / \mathrm{mL})$, and IFN- $\gamma(50 \mathrm{ng} / \mathrm{mL})$ for $48 \mathrm{~h}$. (A) The TUNEL assay was used to analyze cell apoptosis. (B) Human islets were collected and lysed with $50 \mu \mathrm{L}$ lysis buffer. Ten microliters of cell lysate from each group sample were used for caspase 3 activity assay using Ac-DEVD-pNA substrate. Results are expressed as the percentage of control and represented as the mean $\pm \mathrm{SD}, \mathrm{n}=3$. Significance established at $\mathrm{P}<0.05(*)$ and $\mathrm{P}<$ $0.01(* *)$. 

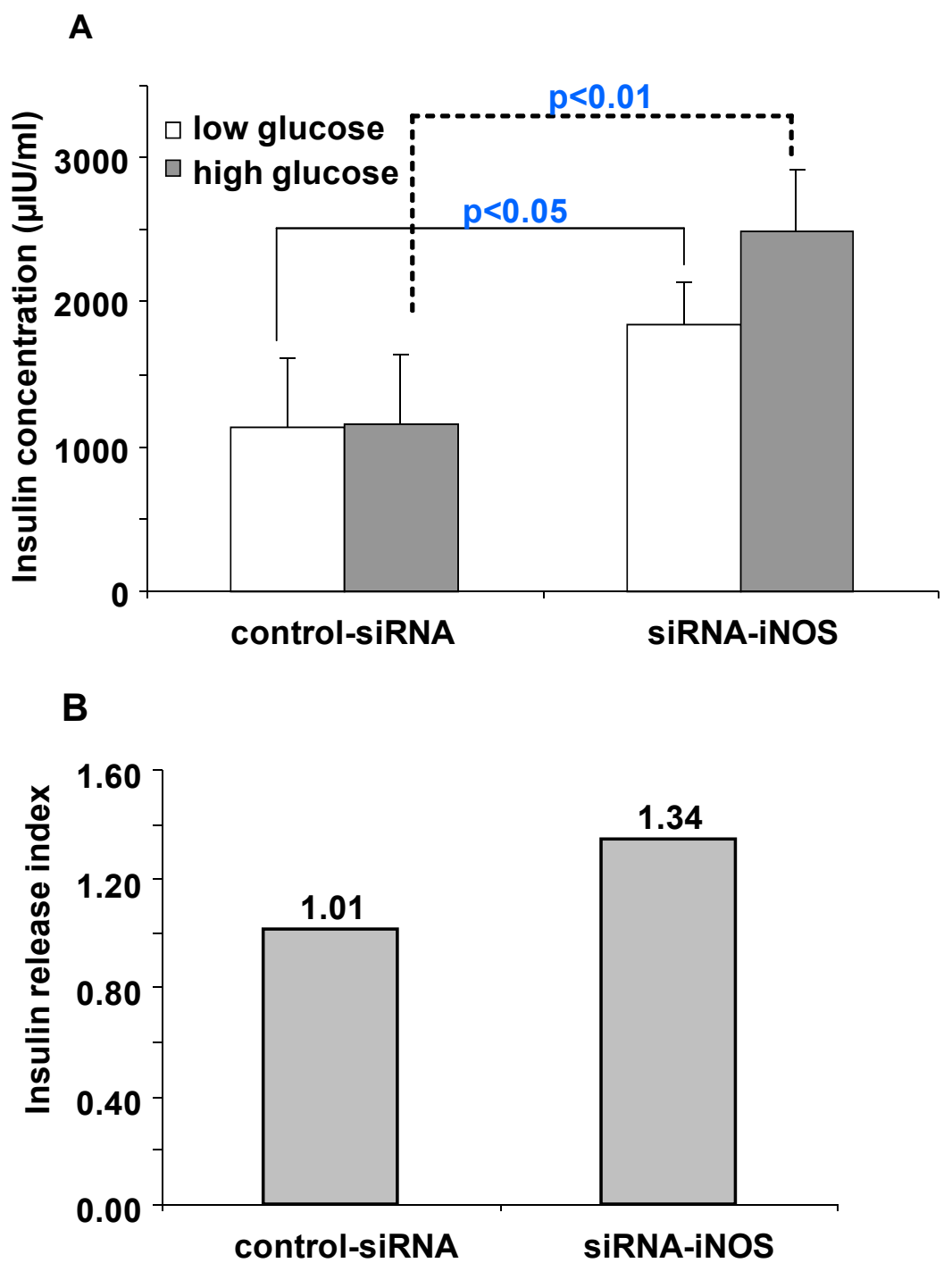

\section{Figure 3.7 Human islet function after iNOS gene silencing.}

At $12 \mathrm{~h}$ post- transfection with Lipofectamine 2000/ siRNA-iNOS or Lipofectamine 2000/siRNA-control complexes, islets were incubated with inflammatory cytokines cocktail of IL-1 $\beta(50 \mathrm{pg} / \mathrm{mL})$, TNF- $\alpha(5 \mathrm{ng} / \mathrm{mL})$, and IFN- $\gamma(50 \mathrm{ng} / \mathrm{mL})$ for $48 \mathrm{~h}$. Then, islets were challenged with two different concentration of glucose $(60$ and $360 \mathrm{mg} / \mathrm{dL})$. Insulin stimulation index (SI) was determined. Results are the average of three replicates. (A) insulin secretion as a function of glucose concentration, (B) stimulation index. $\mathrm{P}<$ 0.05 was considered statistically significant. 
proinflammatory responses. Therefore, we recently determined the effect of chemically synthesized siRNAs against iNOS and NF- $\kappa \mathrm{B}$ on iNOS gene expression, NO production and apoptosis in insulin producing $\beta$-cell line, INS-1E, and demonstrated that silencing of iNOS is more effective than that of NF- $\mathrm{B}$ [116]. Since human islets are clusters of 1002000 cells, it is difficult to achieve high transfection efficiency. Therefore, in present study, we compared the transfection efficiency of lipid/siRNA complexed in rat $\beta$ cells and intact human islets. We first determined the transfection efficiency on INS-1E cells, which showed $95.9 \%$ cells were incorporated with fluorescein-labeled siRNA (Figure 3.1B). This result is consistent with a previously report by Hagerkvist et al [271], who demonstrated a transfection efficiency of $96.2 \%$ in another murine $\beta$ cells line, $\beta$-TC- 6 cells.

For human islets, as shown in Figure 3.4B, the transfection efficiency was $21.5 \%$ and $28.3 \%$ at siRNA concentrations of $100 \mathrm{nM}$ and $400 \mathrm{nM}$ in human islets, which are lower than that reported by Bradly et al,[270] (46.3\% at siRNA concentration of $400 \mathrm{nM})$, but higher than that reported by Hagerkvist et al [271], (10.9\% at siRNA concentration of $100 \mathrm{nM}$ ). This discrepancy may be due to the fact that these authors used mouse islets, while we used human islets. In addition, the difference in transfection protocol may also contribute to the varied transfection efficiency achieved. Nevertheless, this is an interesting finding suggesting that siRNA may penetrate beyond the periphery into a larger percentage of an intact islet cell mass than previously thought. Unlike intact islets, transfection efficiency was $>90 \%$ in dispersed islets, which is close to that observed in cultured $\beta$-cells. However, we prefer to use intact islets, which are a cluster of 200-1000 cells and represent the real situation [271]. Nevertheless, these results shed light on the reasons that cause the different transfection efficiency between intact human islets and $\beta$ cell lines.

It is widely believed that the treatment of proinflammatory cytokine results in $\beta$-cell death possibly due to apoptosis and necrosis $[52,279]$. This cytotoxic effect of cytokines may be mediated by inducing iNOS and subsequently producing radical NO as well as of other free radicals, such as peroxynitrite (OONO-) and superoxide (O2-) [59, 280, 281]. As reported previously [59], nearly $50 \%$ of the gene related to cytokine-induced $\beta$ cell death are NO-dependent, which shows the essential role of iNOS pathway in cytokinemediated cell toxicity. Necrosis and apoptosis are found shortly after rat islet transplantation. Non-specific inflammation at the graft site may contribute to the initial islet damage which results in massive loss of transplanted tissues, and primary non-graft function [282]. iNOS gene expression on islets increased significantly after transplantation. The maximal iNOS gene expression was observed day 1 after transplantation and then declined progressively [48]. Increased iNOS gene expression led to an early inflammatory process in islet transplantation, suggesting iNOS is an important mediator of graft inflammation and islet damage in early islet transplantation [48]. Therefore, we hypothesized that the silencing of iNOS gene might reduce iNOS mediated inflammatory response, and prevent islet cell death.

To determine iNOS gene silencing in human islets, we redesigned three siRNA sequences targeting different regions of human iNOS and used for transfection in human 
islets. As shown in Figure 3.5A, there was around 50\% iNOS gene expression has been silenced by siRNA complexed with Lipofectamine 2000, which is similar to that reported by Bradley et al., [270] who demonstrated 55\% reduction of Ins-2 gene expression by isolated rodent islets. This finding is attractive which demonstrates the possibility to specifically reduce the target mRNA in human islets with siRNA. Various extent of iNOS gene silencing was achieved in present study with different siRNA duplexes targeting different regions of iNOS mRNA, with the highest gene silencing for the siRNA-iNOS691 group compared to the control siRNA group (Figure 3.5A). There was also significant decrease in NO production when islets were transfected with lipid/siRNAiNOS-691 complexes (Figure 3.5B). As reported previously, the gene silencing effect of siRNA varied considerably depends on target sequences [283], and the secondary structure of target region that is essential for siRNA binding [284]. Therefore, a more potent siRNA could be found by designing and screening more siRNA sequences target different regions of mRNA.

Caspases play a key role in apoptosis. In mammals, there are 14 caspases, among them caspase 3 is the major effector caspase involved in apoptosis. It is activated by upstream proteases including caspase 6 , caspase 8 , and cytotoxic T cell-derived granzyme B [65]. The critical role of caspase 3 in cytokine-induced $\beta$ cell apoptosis has been reported [285287]. Both caspase 3 inhibitor (Z-DEVD-FMK) [288] and X-linked inhibitor of apoptosis protein (XIAP) [64] have been shown to prevent apoptosis of transplanted human islets. We observed that siRNA targeting iNOS decreased proinflammatory cytokine-induced caspase 3 activity in human islets (Figure 3.6B). The reduced caspase 3 activity probably contributes to the prevention of human islets from apoptosis.

One of the later steps in apoptosis is DNA fragmentation, which results from the activation of endonucleases during the apoptotic process. The free 3 '-OH strand breaks resulting from DNA degradation can be measured by a TUNEL assay using the APODIRECTTM, which is a single-step staining method for labeling DNA breaks to detect apoptotic cells by flow cytometry analysis. Consistent with the results of caspase 3 activity, TUNEL assay demonstrated the prevention of $\beta$ cell apoptosis on human islet as well as INS-1E cells. As shown in Figure 3.3B, siRNA-iNOS treatment significantly reduced cytokine-induced INS-1E cell apoptosis to $12.3 \%$, compared to the control siRNA treated cells with $42.3 \%$ apoptotic cells. In human islets the protective effect is less than that observed in INS-1E cells. The treatment of siRNA-iNOS decreased the percentage of cell apoptosis from $36.2 \%$ to $28.5 \%$ (Figure $3.6 \mathrm{~B}$ ). The difference in the protective effects can be explained by much lower transfection efficiency achieved in intact human islets compared to that achieved in INS-1E cells (21-28\% VS 95\%; Figure 3.1B and 3.4B). Besides, due to the heterogeneous feature of human islets, we cannot exclude the existence of other mechanisms involved in the apoptosis of human islets [52].

To determine the effect of transfection process on the in vitro islet function, islets were transfected with lipid/siRNA-iNOS and lipid/siRNA-control complexes and then incubated with a cocktail of inflammatory cytokines. The islet function was determined by static incubation of islets with different concentration of glucose. There was increase in insulin release as a function of glucose concentration for the islets transfected with 
lipid/siRNA-iNOS complexes. In contrast, there was little increase in insulin secretion with increase in glucose concentration for the islets transfected with lipid/siRNA-control (Figure 3.7). The results suggest that iNOS gene silencing partly protected human islets from proinflammatory cytokine-mediated destruction. The stimulation index is somewhat lower than what we usually achieve, possibly due to the poor quality of human islets.

Our results demonstrate that silencing of iNOS gene expression results in reduction of NO production and reduced cell death in rodent $\beta$ cell line as well as in human islets. Due to the pivotal role of iNOS in cytokine signal cascade, it is not unexpected that we could prevent proinflammatory cytokine-induced $\beta$ cell death by silencing iNOS gene expression. iNOS gene silencing also partly protected human islets from inflammatory cytokine mediated destruction of glucose stimulated insulin secretion. The present study is the first to demonstrate the prevention of proinflammatory cytokine-induced cell death in human islets with siRNA target iNOS gene. 


\section{CHAPTER 4. BIPARTITE VECTORS FOR CO-EXPRESSION OF A GROWTH FACTOR CDNA AND SHRNA AGAINST AN APOPTOTIC GENE*}

\subsection{Introduction}

RNA interference (RNAi) is an evolutionarily conserved biologic process that regulates gene expression by small interfering double stranded RNA (siRNA) mediated sequencespecific, post-transcriptional gene silencing [21, 22, 269]. Several steps are involved in RNAi: 1) long double-stranded RNA (dsRNA) is processed by DICER into 19-23 base pair siRNA duplex; 2) siRNA duplex is incorporated into a complex named RNAinduced silencing complex (RISC); 3) RISC is activated by eliminating passenger strand of siRNA duplex, and results in mRNA degradation or translational repression [120].

RNAi has been extensively used as a tool for gene knockout in cell lines and animal models [289, 290]. In addition, it is also a promising therapeutic approach for disease therapy [26, 291]. Recently, we have tested the effects of siRNAs against inducible nitric oxide synthase (iNOS) genes on insulin producing $\beta$-cell line (INS-1E) and on human islets $[25,115]$. iNOS gene silencing with chemically synthesized siRNA decreased NO production in INS-1E rat $\beta$-cells and human islets, reduced proinflammatory cytokineinduced $\beta$-cell death, and partially protected the human islet function [115]. We also observed in another study that adenovirus-based shRNA against caspase 3 gene (Advcaspase 3-shRNA) efficiently silenced caspase 3 gene and its gene silencing effect lasted beyond five days, which resulted in the protection of islets from cytokine-induced apoptosis[107].

As an alternative cure for type I diabetes, human islet transplantation has made a great progress, especially after the success of Edmonton protocol [7, 292]. However, a large number of the patient returned to insulin dependent within a year after islet transplantation [293]. The main reason for the failure of islet transplantation is that less than $30 \%$ of the transplanted islets are survived in the early days post-transplantation due to several reasons, including (1) poor revascularization, which results in insufficient supply of oxygen and nutrition; and (2) proinflammatory cytokines-induced islet $\beta$-cell death including apoptosis and necrosis [12]. In our previous study, we investigated the use of bipartite plasmid or Adenoviral vectors which co-express one gene for reducing inflammatory response (e.g. hIL-1Ra) and another for facilitating revascularization (e.g. HGF, VEGF) [18, 294]. This combinatorial approach showed synergistic effect by working on two independent therapeutic targets. Therefore, it is possible to observe improved therapeutic efficacy with bipartite vectors that co-express a vascular endothelial growth factor (VEGF) cDNA and shRNA targeting an apoptotic gene such as iNOS or caspase 3 .

* Adapted with permission. F. Li, R.I. Mahato, Bipartite vectors for co-expression of a growth factor cDNA and short hairpin RNA against an apoptotic gene, J Gene Med, 11 (2009) 764-771. 
In order to co-express two genes in a single vector, we can use two separate expression cassettes driven by two promoters or using internal ribosome entry site (IRES) sequence $[295,296]$. The use of IRES is usually not preferred, since the gene after IRES usually showed lower expression level [297]. Co-expression of a shRNA and a gene with two separate promoters is similar to that of two different genes, but it might be different for co-expression of a shRNA and a gene with a single promoter. Unlike gene expression plasmid which utilizes a Pol-II promoter, shRNA expression can utilize both Pol-II promoters (such as CMV promoter) and Pol-III promoters (such as H1, U6 promoters). IRES sequence is not necessary for co-expressing multiple shRNA or combination of a shRNA and a cDNA within a single promoter [158, 298, 299]. It is has also been reported that co-expression of shRNA and cDNA can be realized by inserting a promoterless shRNA within the intron of a gene [197].

A bipartite vector, which can effectively silence a target gene and expresses a therapeutic gene properly, is in great need for treating various diseases including diabetes, cancer and viral infection. siRNA targeting sequence and promoters used will greatly influence gene silencing efficiency. A shRNA and cDNA could be expressed by two different promoters or by a single promoter. It was also reported that the miRNA-based shRNA showed improved gene silencing than conventional shRNA $[152,153]$. In this study, we have systemically investigated these factors to find a most optimal bipartite vector and indentify parameters defining a potent shRNA and gene co-expression vector.

\subsection{Materials and Methods}

\subsubsection{Materials}

Fetal bovine serum (FBS) was purchased from Mediatech, Inc (Herndon, VA). Penicillin/streptomycin, phosphate-buffered saline (PBS), 0.25\% (w/v) trypsin-EDTA and DMEM medium were purchased from GIBCO-BRL (Gaithersburg, MD). All oliginucleotides used for shRNA cloning were obtained from Integrated DNA Technology (Coralville, IA). All the enzymes used in cloning were purchased from New England Biolabs (Ipswich, MA).

\subsubsection{Plasmids}

Negative control shRNA plasmid and Human iNOS cDNA plasmid (p-iNOS) were purchased from OriGene (Rockville, MD). Plasmids encoding shRNA targeting five different regions of iNOS gene were purchased from Open Bioystems (Huntsville, AL). To generate p-H1-shiNOS-CMV-GFP, p-U6-shiNOS, and p-CMV-shiNOS, two shRNA oligos ( synthesized by IDT DNA) were annealed and cloned into pRNAT-H1.1/shuttle (BamHI, HindIII), pSIREN-Shuttle (BamHI, EcoRI) and p-shuttle2 (XbaI+AflII), respectively. p-H1-shiNOS-CMV-VEGF was generated by replacing GFP gene with VEGF PCR fragment from p-CMV-VEGF165. Briefly, p-H1-shiNOS-CMV-GFP was 
digested with PflmI, followed by treating with Klenow enzyme. After purification, it was digested with NheI and then purified. VEGF gene was amplified with PCR using following primer: Forward (Nhe I) GCCTAGCTAGCTAGATGAACTTTCTGCTGTCTTG; Reverse (DraI) CGCTATTTAAATCACCGCCTCGGCTTGTCACATC. To make p-U6-shiNOS-CMVVEGF, pSIREN-shuttle was digested with I-ceu and Bam HI, and the fragment with U6 promoter was sub-cloned into I-Ceu and Bam HI site in p-H1-shiNOS-CMV-VEGF.PU6-mir375-shiNOS was generated by cloning annealed mir375 oligos into pSIRENshuttle (BamHI,EcoRI). To make p-U6-mir30-shiNOS, mir30-shiNOS sequence was synthesized and sub-cloned into pSIREN-Shuttle (BamHI, EcoRI). mir30-shiNOS sequence:GGATCCGTGCTCGCTTCGGCAGCACATATACTAGTCGACTAGGGATA ACAGGGTAATTGTTTGAATAGGCTTCAGTACTTTACAGAATCGTTGCCTGCAC ATCTTGGAAACACTTGCTGGGATTACTTCTTCAGGTTAACCCAACAGAAGGG CGGCCGCAAGGTATATTGCTGTTGACAGTGAGCGCGTGTATTTAACTGCCTTG TGTAGTGAAGCCACAGATGTACACAAGGCAGTTAAATACACATGCCTACTGC CTCGTCTAGAAAGGGGCTACTTTAGGAGCAATTATCTTGTTTACTAAAACTGA ATACCTTGCTATCTCTTTGATACATTTTTTGaattc. To make p-CMV-mir30shiNOS, mir30-shiNOS sequence was amplified with PCR using following primer: forward (Dra I) GTATTTAAAGGATCCGTGCTCGCTTCGGC; reverse (Afl II) CGCCTTAAGAATGTATCAAAGAGATAGCA and PCR product was cloned into pshuttle2 (DraI, AflII) after restriction enzyme digestion. p-CMV-VEGF-mir30-shiNOS was made by inserting VEGF PCR fragment between CMV promoter and mir30 shiNOS sequence in p-CMV-mir30-shiNOS using NheI and DraI sites. shRNA sequences are listed in supplementary information. All the plasmids were purified by Promega miniprep kit and confirmed by DNA sequencing.

\subsubsection{Cell culture and transfection}

AD-293 cells were cultured in DMEM supplemented with $10 \% \mathrm{FBS}, 100 \mathrm{U} / \mathrm{mL}$ penicillin, and $100 \mathrm{mg} / \mathrm{ml}$ streptomycin. In transfection experiments, cells were seeded in a 48 -well plate at a density of 20,000 cells per well $24 \mathrm{~h}$ before transfection. Then, 0.05 $\mu \mathrm{g}$ iNOS cDNA plasmid and $0.3 \mu \mathrm{g}$ shRNA plasmid were co-transfected into cells with Fugene HD transfection reagent (Roche applied science, Indianapolis, IN).

\subsubsection{Real time RT-PCR}

Human iNOS and VEGF expression were determined at mRNA level by real time RTPCR. Following treatment, total RNA was extracted with RNeasy Mini KIT and treated with DNase by on-column digestion (RNase-Free DNase Set). RNA concentration was determined by spectrophotometer (NanoDrop). One hundred and seventy nanograms total RNA was converted into cDNA using multiscribe reverse transcriptase reagents and random hexamers at a $10 \mu \mathrm{l}$ reaction system. Two microliters of cDNA were used as a template and analyzed by SYBR Green-I dye universal PCR master mix on LightCycler 480 Instrument. The primers used for real-time PCR were as follows: human iNOS: 
Forward 5'-ACGTGCGTTACTCCACCAACA-3'; Reverse 5'-

CATAGCGGATGAGCTGAGCA-3'(amplicon size $102 \mathrm{bp}$ ); human ribosomal protein S19 (human S19); Forward 5' GCTTGCTCCCTACCGATGAGA-3; Reverse 5'ACCCCGGAGGTACAGGTG-3'(amplicon size $73 \mathrm{bp}$ ) [115]. The primer for VEGF gene was the same as previously described [18].To assess the specificity of the amplified PCR product, the Melting Curve Analysis was performed on the LightCycler 480 Instrument. The results iNOS mRNA level were compared by calculating the $\mathrm{CP}$ value and normalized by the reference genes (rat 18S or human S19).

\subsubsection{Determination of nitric oxide production}

Nitric oxide (NO) is rapidly oxidized in culture medium into nitrite, which accumulates in the sample and can be easily correlated with NO production. Therefore, nitrite concentration was determined using the Griess assay (Promega, Madison, MI). Fifty microliters of cell culture supernatant was added to a 96 well-plate and mixed with $50 \mu \mathrm{L}$ of $1 \%$ sulfanilamide in 5\% phosphoric acid solution and incubated for $5 \mathrm{~min}$ at room temperature in the dark. Then, $50 \mu \mathrm{L}$ of $0.1 \% \mathrm{~N}-1$-naphthylethylenediamine dihydrochloride (NED) aqueous solution was added to each well. The plate was incubated for an additional $10 \mathrm{~min}$, and absorbance was measured at $560 \mathrm{~nm}$ using a microplate reader. To determine the nitrite concentration in each sample, a standard curve was prepared using nitrite standard solution and culture medium as matrix.

\subsubsection{Determination of VEGF expression from bipartite plasmids with ELISA}

At $36 \mathrm{~h}$ after transfection of AD-293 cells with $0.3 \mu \mathrm{g}$ per well plasmids, culture medium of AD-293 cells were collected. The VEGF concentration in the culture medium was measured by ELISA according to the manufacture's protocol (R\& D systems, Minneapolis, MN).

\subsection{Results}

\subsubsection{Effect of targeting sequence on iNOS gene silencing}

Targeting sequence is one of the most important elements for effective gene silencing. Therefore, we first screened five plasmids encoding shRNA targeting different regions of human iNOS gene. The targeting sequences of different plasmids are listed in Figure 4.1A. To find the most potent targeting sequence, plasmids expressing shRNA against five different iNOS mRNA regions were co-transfected with p-iNOS into AD-293 cells. Plasmid with scrambled sequence was used as a control. At 24 hours after transfection, NO production from iNOS gene expression was measured as nitrite by Griess Assay. As demonstrated in Figure 4.1B, all five shRNA expression plasmids were able to silence iNOS expression and among them the 4060 showed $66 \%$ reduction of NO production. 

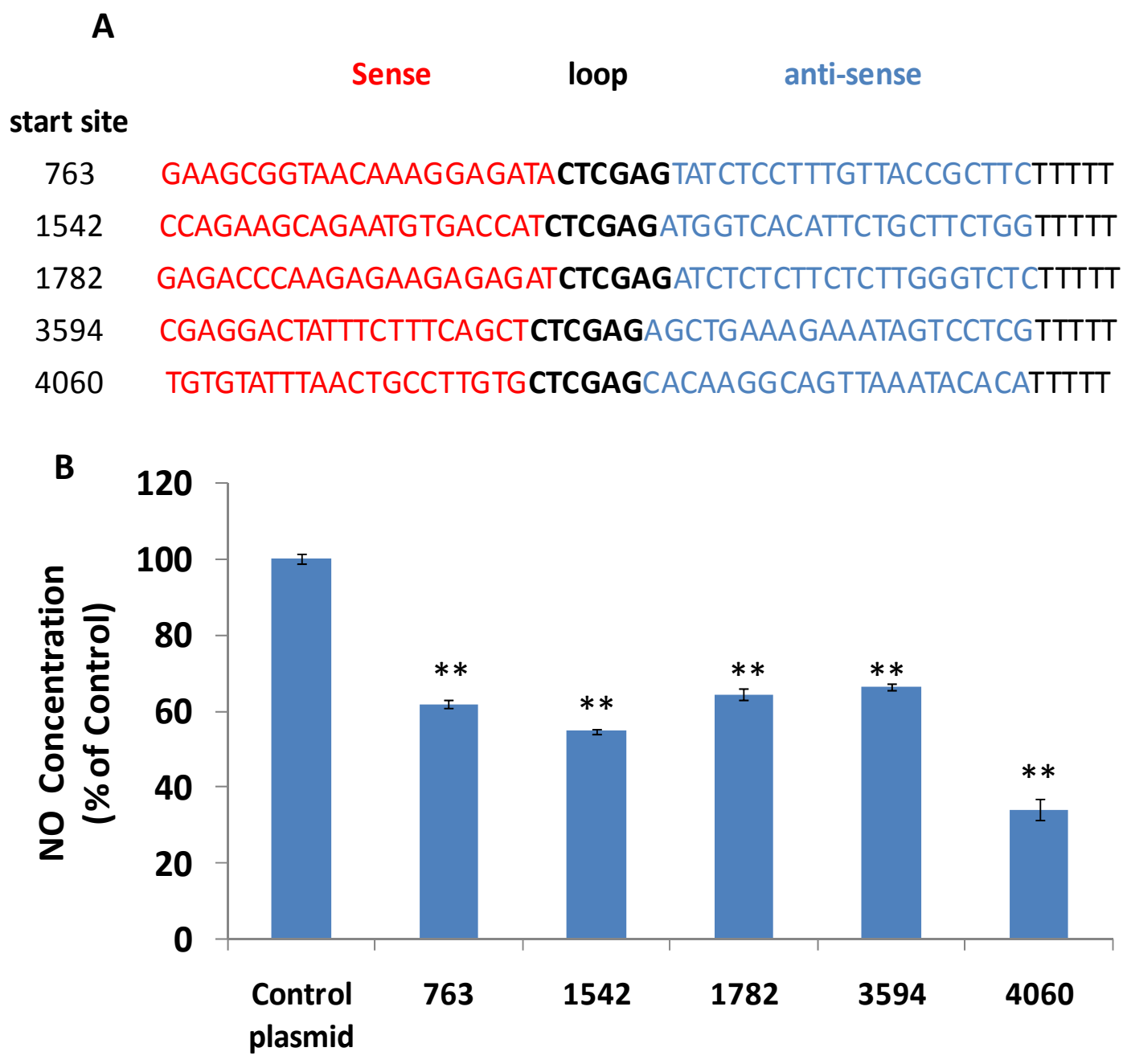

Figure 4.1 Effect of targeting sequence on iNOS gene silencing.

(A) Sequences of shRNA against different regions of human iNOS gene (NM_000625). (B) Plasmids with different shRNA sequences as well as control plasmid were cotransfected with piNOS into AD293 cells. At $24 \mathrm{~h}$ after transfection, NO production was measured by Griess Assay. Nitrite concentration is expressed as the percentage of control. Results are the mean $\pm \mathrm{SE}(\mathrm{n}=4)$. ${ }^{* *}$ indicates $\mathrm{P}<0.01$ compared with control plasmid group. 
Therefore, the targeting sequence 4060 was used in the following experiments for shRNA-based or miRNA-based shRNA construction.

\subsubsection{Effect of promoters on iNOS gene silencing}

Promoter is an important regulatory element in gene expression. Among several promoters, H1, U6 and CMV promoters are most widely used for shRNA mediated gene silencing. Since some discrepancies have been reported for the efficiency of these promoters $[125,153]$, we compared the efficiency of these three promoters for shRNA mediated iNOS gene silencing by constructing iNOS shRNA expression plasmids with H1, U6 and CMV promoters (Figure 4.2A). After co-transfection with p-iNOS plasmid into AD-293 cells, we found that iNOS shRNA expression plasmids could effectively (p $<0.01$ ) reduce the NO production by 69,62 , and $60 \%$ for $\mathrm{H} 1, \mathrm{U} 6$ and CMV, respectively (Figure 4.2B). This suggests that there is no significant difference among these three promoters in iNOS gene silencing.

\subsubsection{Effect of VEGF co-expression on iNOS gene silencing}

Co-expression of shRNA and therapeutic proteins is a promising combinatorial RNAi strategy [185], which will have synergistic effect by acting on different targets. However, it is not sure whether the over-expression of VEGF gene under a strong CMV promoter will interfere with the expression and processing of shRNA. As illustrated in Figure 4.3A, a plasmid has been constructed to co-express VEGF cDNA and iNOS-shRNA. VEGF cDNA was under the control of CMV promoter, while iNOS-shRNA was driven by U6 promoter. As shown in Figure 4.3B, p-U6-shiNOS-CMV-VEGF and p-U6-shiNOS reduced the NO production by $59 \%$ and $62 \%$, respectively $(\mathrm{p}<0.01)$. In contrast, the plasmid expressing VEGF did not shown significant reduction of NO production. It was also noticed that there was no significant difference between p-U6-shiNOS and p-U6shiNOS-CMV-VEGF. Therefore, the co-expression of VEGF cDNA and iNOS-shRNA will not have much interference with the shRNA-mediated gene silencing.

\subsubsection{Effect of mir375 and mir30 backbones on iNOS gene silencing}

Efficient gene silencing has been reported when shRNA was embedded in miRNA backbone $[153,154]$. To find out the possibility of increasing iNOS gene silencing effect by shRNA with miRNA backbone, two shRNA were designed based on mir375 and mir30 structures (Figure 4.4A). As shown in Figure 4.4C, significant reduction of NO production were observed in all of three vectors tested, p-U6-shiNOS (reduced by $62 \%, \mathrm{p}$ $<0.01$ ), p-U6-mir375-shiNOS (reduced by 39\%, p < 0.01) and p-U6-miR30-shiNOS (reduced by $30 \%, p<0.01$ ). In addition, $\mathrm{p}$-U6-shiNOS is significantly more efficiently than $\mathrm{p}$-U6-mir375-shiNOS or $\mathrm{p}-\mathrm{U} 6-\mathrm{mir} 30$-shiNOS $(\mathrm{p}<0.01)$. Gene silencing efficiency of these three shRNA vectors was further investigated at mRNA levels by real-time PCR (Figure 4.4B). All of these three vectors reduced NO mRNA expression significantly 
A
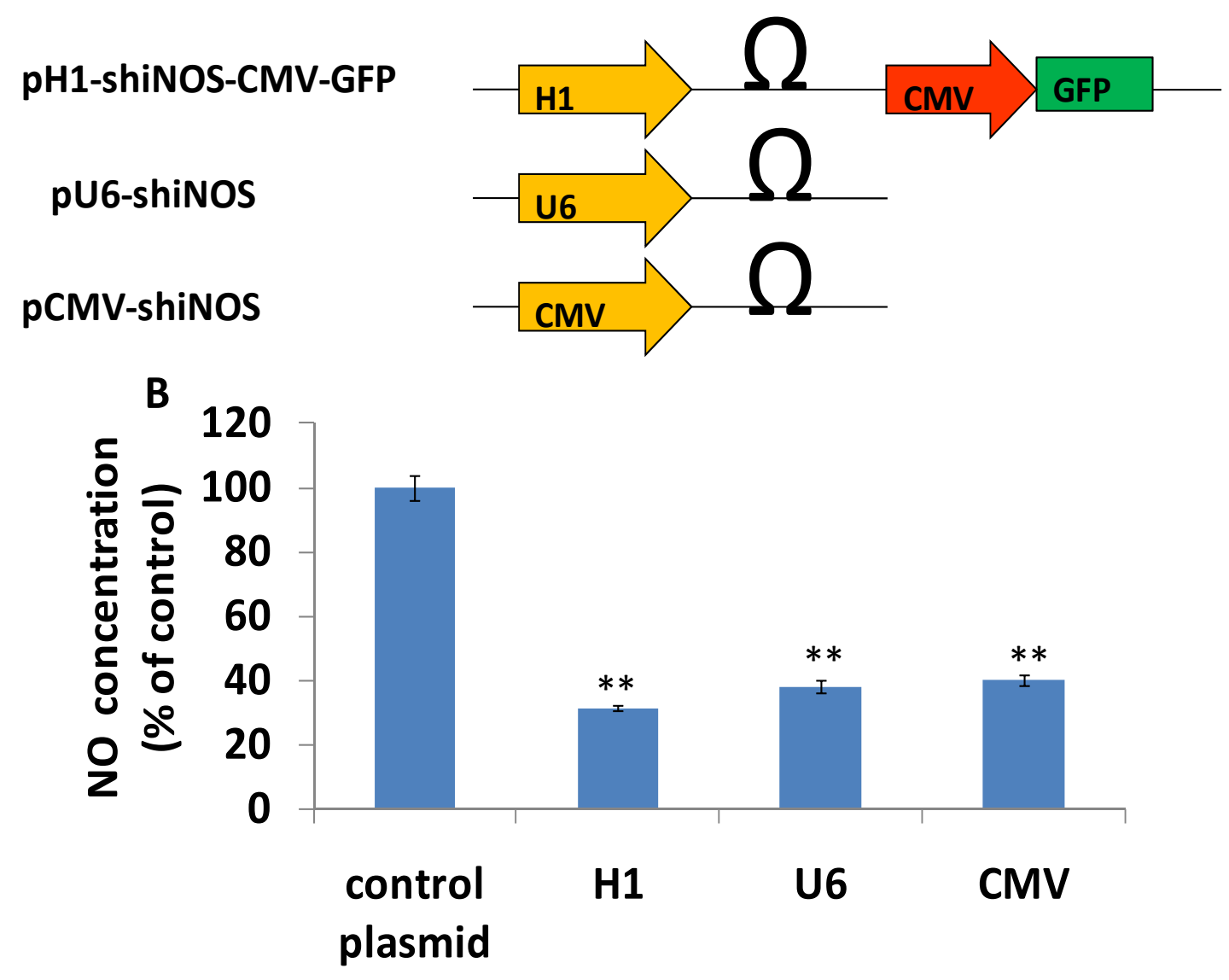

Figure 4.2 Effect of different promoters on iNOS gene silencing.

(A) Structure of pH1-shiNOS-CMV-GFP, pU6-shiNOS and pCMV-shiNOS. (B) Plasmids with different promoters (H1, U6 and CMV) as well as control plasmid were co-transfected with piNOS into AD293 cells. At 24 hours after transfection, NO production was measured by Griess Assay. NO concentration is expressed as the percentage of control. Results are the mean $\pm \operatorname{SE}(n=4)$. ** indicates $\mathrm{P}<0.01$ compared with control plasmid group. 
A

pU6-shiNOS
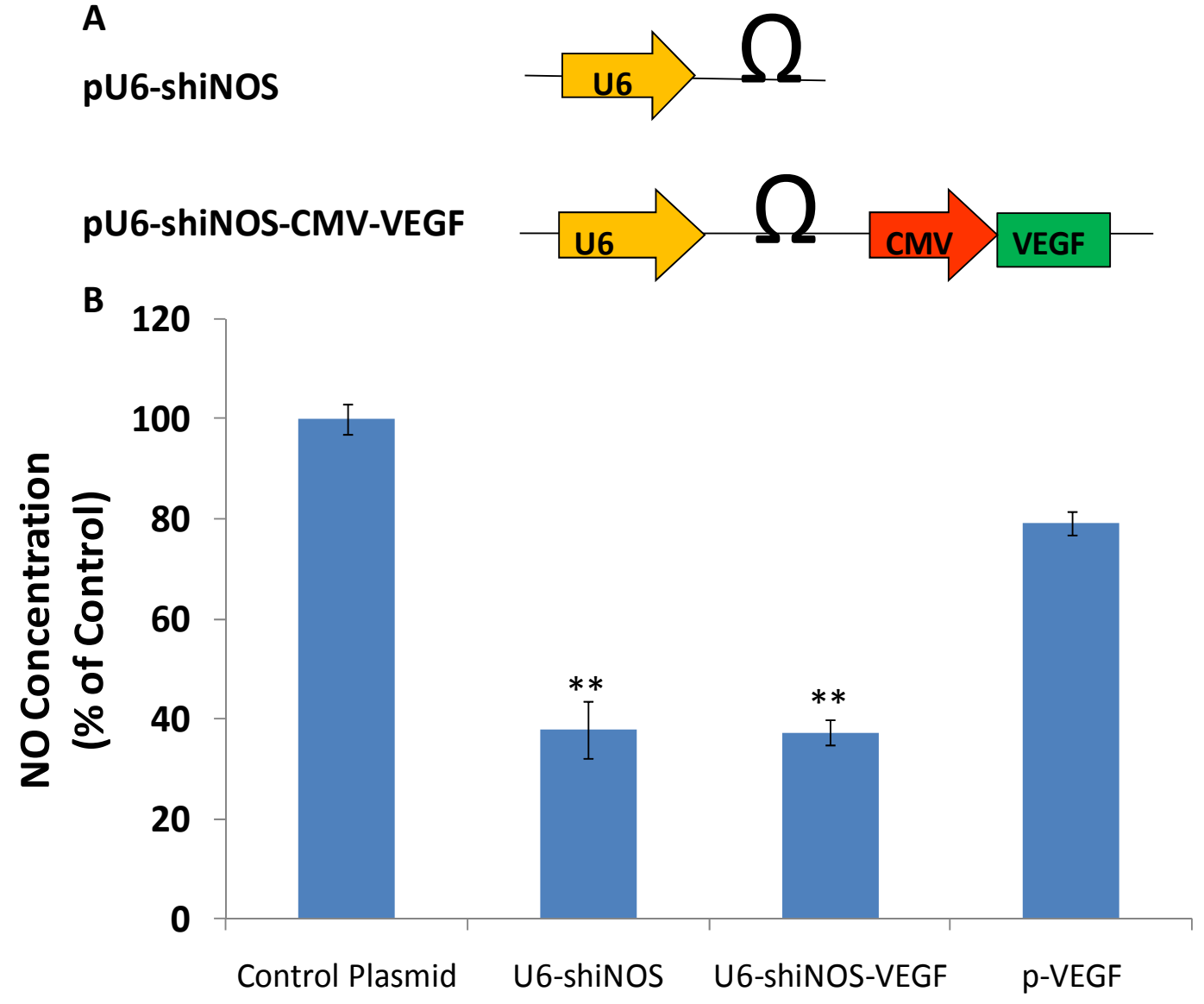

Figure 4.3 Effect of VEGF gene co-expression on iNOS gene silencing.

(A) Structure of pU6-shiNOS, and pU6-shiNOS-CMV-VEGF. (B) Plasmids coexpressing shRNA and VEGF, plasmid expressing VEGF alone, as well as control plasmid were co-transfected with piNOS into AD293 cells. At $24 \mathrm{~h}$ after transfection, NO production was measured by Griess Assay. Nitrite concentration is expressed as the percentage of control. Results are the mean $\pm \operatorname{SE}(n=4)$. ** indicates $p<0.01$ compared with control plasmid group. 

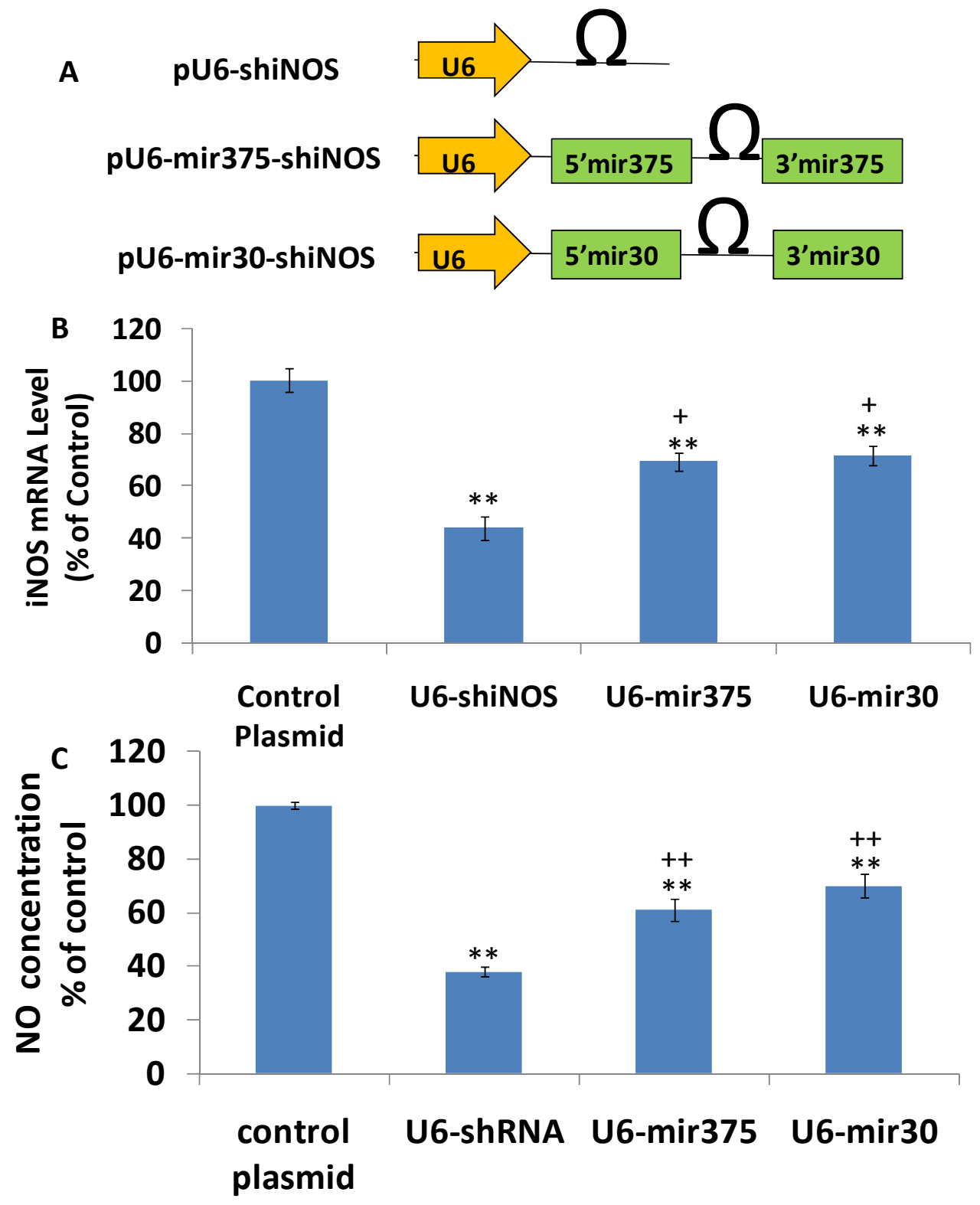

Figure 4.4 Effect of mir375 and mir30 backbone on iNOS gene silencing.

(A) Structure of pU6-shiNOS, pU6-mir375-shiNOS and pU6-mir30-shiNOS. Plasmids with different backbones as well as control plasmid were co-transfected with piNOS into AD-293 cells. At 24 hours after transfection, (B) iNOS mRNA levels was measured by Real-time PCR, (C) NO production was measured by Griess Assay. NO concentration is expressed as the $\%$ of control. Results are the mean $\pm \mathrm{SE}(\mathrm{n}=4)$. ${ }^{* *}, \mathrm{p}<0.01$ compared with control plasmid group; $++, \mathrm{p}<0.01,+, \mathrm{p}<0.05$, respectively, compared with $\mathrm{pU6}$ shiNOS group. 
( $\mathrm{p}<0.01$, compared with control). In addition, $\mathrm{p}-\mathrm{U} 6$-shiNOS (reduced by $56 \%$ ) is significantly more efficiently than p-U6-miR375-shiNOS (reduced by $30 \%$ ) or p-U6miR30-shiNOS (reduced by 28\%) ( $<<0.05$, compared with $\mathrm{p}-\mathrm{U} 6$-shiNOS).

4.3.5. Inserting VEGF gene between CMV promoter and mir30-shRNA enhanced iNOS gene silencing

We have shown the successful co-expression of iNOS shRNA and VEGF cDNA under two expression cassettes. To further investigate the possibility of co-expressing VEGF cDNA and iNOS shRNA under a single promoter, a plasmid with mir30-shiNOS under the control of CMV promoter was constructed and a VEGF cDNA was inserted between CMV promoter and mir30-shiNOS (Figure 4.5A). As shown in Figure 4.5B, a 39\% reduction in NO production was achieved with $\mathrm{p}-\mathrm{CMV}$-mir30-shiNOS $(\mathrm{p}<0.01$, compared with control plasmid), which is close to that of p-U6-mir30-shiNOS (30\%). However, the gene silencing effect was significantly enhanced by inserting a VEGF cDNA between CMV promoter and mir30-shRNA. In contrast, $61 \%$ reduction in NO production was achieved with $\mathrm{p}-\mathrm{CMV}-\mathrm{VEGF}-\mathrm{mir} 30$-shiNOS $(\mathrm{p}<0.01$, with $\mathrm{p}$-mir30shiNOS).

\subsubsection{Expression of VEGF from bipartite plasmids}

Not only potent silencing of proapoptotic gene, but also sufficient expression of VEGF is essential. Therefore, we measured VEGF gene expression from two bipartite plasmids, pU6-shiNOS-CMV-VEGF and p-CMV-VEGF-mir30-shiNOS. We measured VEGF gene expression by ELISA of the cell culture medium. VEGF concentration was around 65 $\mathrm{ng} / \mathrm{mL}$ for all above plasmids and there is no significant difference among these two plasmids (Figure 4.6B). The VEGF gene expression from these two bipartite plasmids was also measured at mRNA levels with real-time PCR by using VEGF gene specific primers (Figure 4.6A). The results indicated VEGF mRNA levels in these two plasmids treated groups were $914 \pm 18$ and $922 \pm 35$ folds high than that in control group, respectively.

\subsection{Discussion}

Co-transfection approach for evaluating gene silencing effect has been extensively reported $[125,152,155,300]$, which allowed researchers to measure the gene silencing effect with good convenience and reliability. Therefore, a co-transfection of p-iNOS plasmid was used in this study to investigate gene silencing effect of shRNA iNOS vectors with different gene target sequence, promoters, backbones we have constructed. The use of a stably transfected cell line has also been reported [136]. A C6 cell stably expressing firefly luciferase gene was used to study the gene silencing effect shRNAs vectors against firefly luciferase gene. However, this is not appropriate for iNOS gene silencing. iNOS is up-regulated only after stimulation with cytokines or other reagents. 

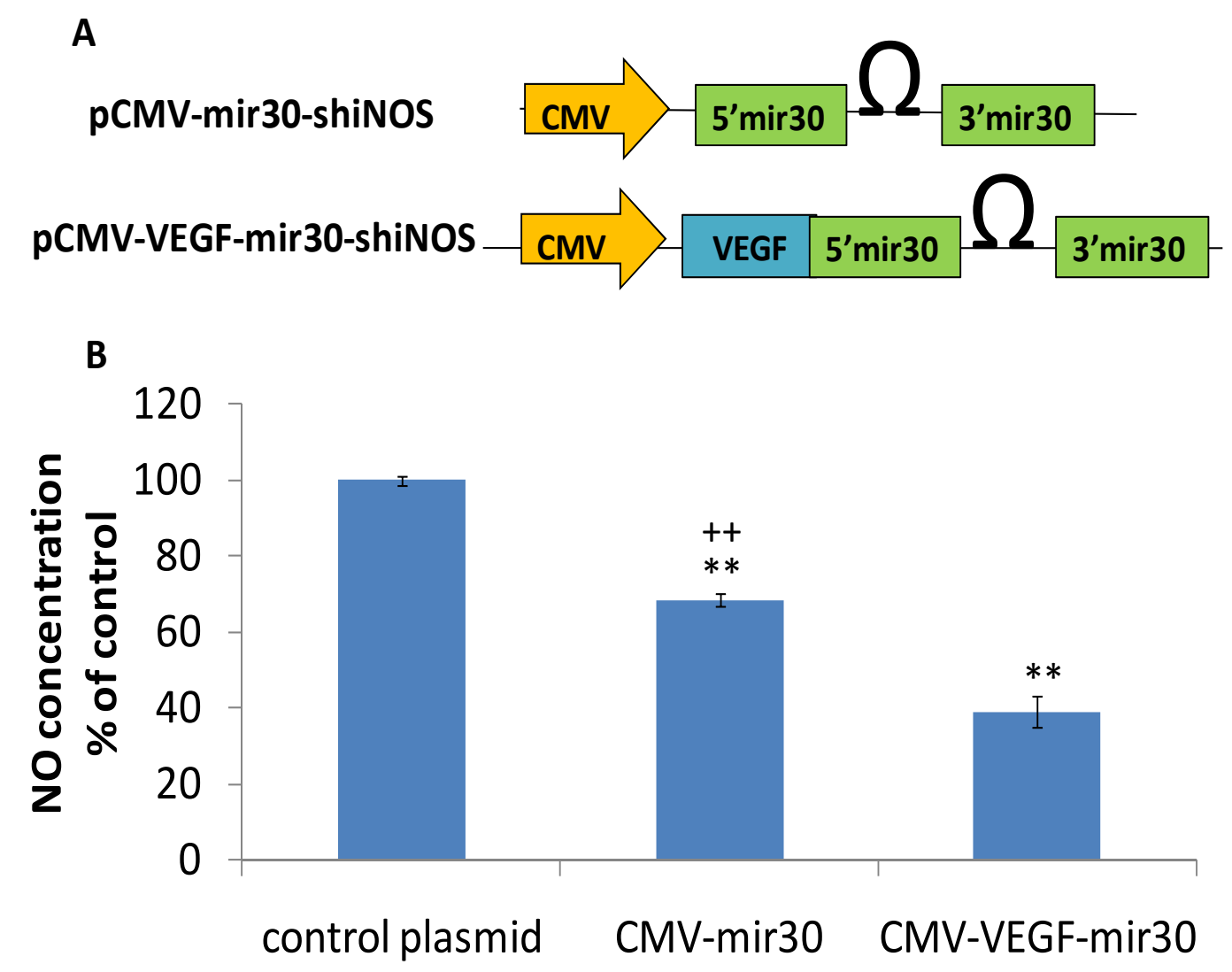

Figure 4.5 Insertion of VEGF gene between CMV promoter and mir30-shRNA enhanced iNOS gene silencing.

(A) Structure of pCMV-mir30-shiNOS and pCMV-VEGF-mir30-shiNOS. (B) pCMVmir30-shiNOS, pCMV-VEGF-mir30-shiNOS as well as control plasmid were cotransfected with piNOS into AD-293 cells. At $24 \mathrm{~h}$ after transfection, NO production was measured by Griess Assay. Nitrite concentration is expressed as the \% of control. Results are the mean $\pm \mathrm{SE}(\mathrm{n}=4)$. ${ }^{*}$ indicates $\mathrm{p}<0.01$ compared with control plasmid group; ++ indicates $\mathrm{p}<0.01$ compared with $\mathrm{pCMV}-\mathrm{VEGF-mir30}$ group. 


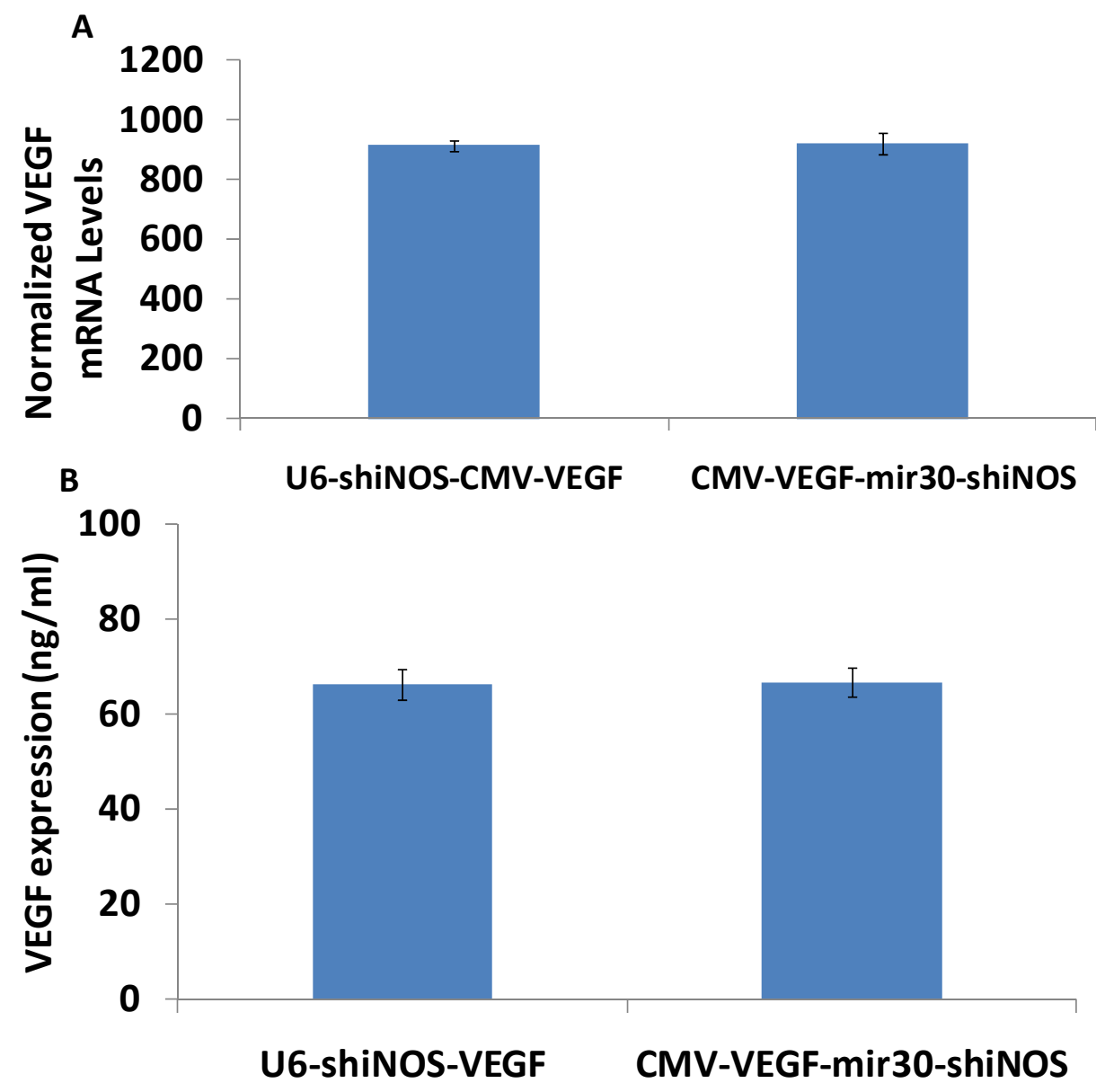

Figure 4.6 Expression of VEGF from pU6-shiNOS-CMV-VEGF and pCMVVEGF-mir30-shiNOS.

pU6-shiNOS-CMV-VEGF and pCMV-VEGF-mir30-shiNOS was transfected into AD293 cells. (A) At 24 hours after transfection, VEGF gene expression levels were measured by Real-time PCR, (B) At $36 \mathrm{~h}$ after transfection, VEGF expression was measured with ELISA. Results are the mean \pm SE $(n=4)$. 
By using co-transfection approach, the iNOS mRNA was expressed from p-iNOS plasmid after transfection. This could mimic the scenario of cytokine-induced iNOS gene much better than iNOS stable expressing cell lines, in which iNOS gene was constantly over-expressed. In addition, there are several limitations for the using of stable cell lines: 1) the establishment of a stable cell line usually takes a long time (at least several weeks); 2) a cDNA expressing vector with an antibiotics resistance gene (eg. Neomycin, puromycin); 3) Only a gene that is not toxic or will not interfere with the vital cellular process could be used to make a stable cell line. Because of above mentioned reasons, a co-transfection method would be a good approach for evaluation gene silencing effect during the development of gene silencing vectors.

The selection of potent targeting sequence that leads to effective gene silencing still remains the key issue for practical application of RNAi technique for disease therapy. Web-based computer programs with different algorithms for designing siRNA and shRNA are available. To increase the chance of finding a potent sequence, empirical rules usually incorporated in these algorithms. These empirical rules includes: thermodynamic property; length of siRNA target; GC content; and RNA secondary structure [121, 126, 301-303]. However, the selection of targeting sequence is still an empiric process and depends on experimental screening of potential targets. This is because our understanding of RNAi mechanism is still insufficient. In addition, most of the algorithms for shRNA sequence design are actually that of siRNA design and convert the siRNA into shRNA sequence. In our study, we have converted potent siRNA targeting sequence into shRNA sequence with success [184, 304]. However, we also observed loss of silencing effect after converting potent siRNA sequence into shRNA (data not show). A better understanding of RNAi mechanism is required for improved design of shRNA. In this study, five shRNA expression plasmids were purchased and screened to find the potent shRNA targeting sequence. In practical, this might be an efficient way to find a potent shRNA for certain application.

Promoter is another important element that determines the duration, intensity and specificity of gene expression [305-307]. The activity of several promoters including tRNA, H1, U6, CMV, LTR, CMV enhancer/H1 has been investigated [136, 153, 299]. In this study, we did not observe significant difference in the levels of iNOS gene silencing when CMV, U6, and $\mathrm{H} 1$ promoters were used for driving shRNA-iNOS. Similar results have been reported by other research groups, where they found that when a less efficient luciferase shRNA sequence was used, there were some differences in luciferase gene silencing among different promoters; with $\mathrm{H} 1$ promoter being less efficient than CMV and U6 promoters. However, when a potent luciferase shRNA sequence was used, the difference in gene silencing effect among different promoters became minimal [153].

The use of miRNA-shRNA in siRNA expression vector is an attractive strategy, which mimics the structure of natural occurring miRNA and thus can be processed by cellular miRNA machinery more efficiently compared with conventional shRNA [153]. Almost $80 \%$ more effective in reducing HIV p24 antigen production was achieved with TAT shRNA using mir-30 backbone compared with conventional shRNA [152]. shRNA is expressed in high level and yields an abundance of precursor, while miRNA-based 
shRNA is expressed at low levels but processed more efficiently [300]. In order to further improve the silencing effect, we designed two shRNAs-based the mir375 and mir30 sequences. The gene silencing potency of mir375-shRNA and mir30-shRNA was compared with that of conventional shRNA. However, the shRNA showed best gene silencing effect ( $62 \%$ reduction of NO production), while mir375 or mir30 shRNA are less potent with $39 \%$ and $30 \%$ reduction of NO production, respectively. Similar findings have been reported by other groups $[125,155]$. Li et al., have compared conventional shRNA and mir30-based shRNAs against luciferase gene or mouse tyrosinase. Among 14 different targeting sequences against luciferase tested, in 11 sequences the conventional shRNA showed better silencing effect than mir30-based shRNA. All the conventional shRNA with 10 different targeting sequences against mouse tyrosinase showed significantly better silencing effect than mir30-based shRNA counterpart. They explained that the shRNA structure used previously for comparison [153] which has a 29-nt stem and a 4-nt loop is not a optimal design for shRNA. When a 4-nt loop sequence was used in shRNA, an insufficient processing of shRNA by Dicer was observed which resulted in poor gene silencing [125]. However, when a 9-nt loop (UUCAAGAGA) and 19-nt stem structure was used in shRNA design, a better gene silencing was observed in conventional shRNA compared with mir30-based shRNA. Therefore, it is reasonable observe better gene silencing effect in shRNA used in our study which has a 21-nt stem and 6-nt loop (CTCGAG). Boudreau et al. also demonstrated in their work that optimized shRNAs are more potent than mir30-based shRNAs for silecing of three genes including GFP,SCA 1 and HD, when the variables were minimized in the comparision [155]. For our study, the ultimate goal is to develop a vector for therapeutic purpose, thus the conventional shRNA which showed better iNOS gene silencing effect will be used in our future study.

Another important feature of miRNA-based shRNA is that they are more amendable to pol II transcription and polycistronic strategies, allowing delivery of multiple siRNA sequences (or a siRNA sequences and a cDNA) with a single promoter [158, 198, 298]. We first designed a miRNA30-based shRNA plasmid p-CMV-mir30-shiNOS, but it was not as potent as we expected. However, when we inserted a VEGF cDNA between CMV promoter and mir30-shiNOS sequence (p-CMV-VEGF-mir30-shiNOS), the gene silencing effect was significantly increased. This result is consistent with the work of Stegmeier et al.,[154] who reported that the insert of GFP, dsRED and Neo genes between CMV promoter and mir30-shRNA cassette increased the knock down of Rb gene. The exact reason for this is still unknown. Probably certain space between CMV promoter and mir30-shRNA is necessary for efficient gene silencing. The p-CMVVEGF-mir30-shiNOS could be another bipartite vector for therapeutic application, which co-expresses shRNA and a cDNA with a single promoter.

The purpose of this study was to construct bipartite plasmids that could co-express VEGF gene for promoting revascularization and shRNA against iNOS gene to reduce human islet $\beta$-cell death. This combinatorial strategy will help to improve the survival and function of islet graft by promoting revascularization and inhibiting apoptosis [12]. The reason for using bipartite plasmids is that it will reduce the use of total plasmid backbone, thus minimizing the immunogenic effect caused by bacterial plasmids [294]. In addition, 
the bipartite plasmids were specially designed as a shuttle plasmid for construction of adenoviral vectors. Moreover, two restriction enzyme sites have been preserved flanking shRNA sequence in our bipartite vector. This feature will allow us to replace the shRNA sequence with a new one through directional cloning. We could conveniently construct $\mathrm{p}-$ U6-shCas3-CMV-VEGF by replacing shRNA sequence in p-U6-shiNOS-CMV-VEGF and caspase 3 gene silencing was observed (data not shown.).

In conclusion, we have constructed bipartite plasmids which co-express VEGF cDNA and shRNA against proapoptotic genes, e.g. iNOS gene. VEGF cDNA and shRNA were driven by two different promoters or by one single promoter. These plasmids are properly designed, which allows us to conveniently change shRNA sequence. In addition, all of these plasmids could be used as a shuttle plasmid for producing adenoviral vectors. 


\section{CHAPTER 5. SYNTHESIS, FORMULATION AND IN VITRO EVALUATION OF A NOVEL MICROTUBULE DESTABILIZER, SMART-100*}

\subsection{Introduction}

Several microtubule-targeting drugs, including paclitaxel and docetaxel, have been developed for treating breast, prostate and non-small-cell lung cancers with great success [308-312]. However, their anticancer effects are diminished because of their intrinsic or acquired drug resistance, which involves the over-expression of P-glycoprotein (P-gp), multidrug resistance protein 7 (MRP 7) and the $\beta$ III-tubulin isotype [313-316]. Therefore, the development of novel anticancer drugs that can circumvent MDR will contribute significantly to the progress of cancer chemotherapy. Ixabepilone (IXEMPRA), an epothiline analog, has recently been approved the FDA to be used as a monotherapy or in combination with capecitabine for treating patients with metastatic or locally advanced breast cancer resistant to treatment with an anthracycline and a taxane, or whose cancer is taxane resistant and for whom further anthracycline therapy is contraindicated [317].

Recently, we have also we have synthesized a series of substituted methoxybenzoyl-arythiazole (SMART) with good anticancer activity on different cancer cell types, including leukemia, non-small cell lung cancer, colon cancer, CNS cancer, renal cancer, melanoma, ovarian cancer, prostate cancer, and breast cancer [318]. The SMART compounds work as microtubule destabilizers by inhibiting tubulin polymerization. Compared with other microtubule-targeting drugs [316], SMART drugs are much easier to synthesize, which will reduce the manufacturing cost and thus make them more affordable to patients.

Although SMART compounds demonstrated excellent anticancer bioactivities, their use was limited due to their poor intrinsic aqueous solubility. Two possible approaches could be used to address this problem. One is chemical modification, which may involve an extensive structure activity relationship (SAR) study. Another way is to improve the solubility of SMART drugs through formulation approaches. Conventionally, dimethyl sulfoxide (DMSO), Cremophor EL, polysorbate 80 (Tween 80) or other surfactants are commonly used to solubilize hydrophobic drugs such as taxane. However, the use of these solubilizing agents is usually associated with liver and kidney toxicity, hemolysis, acute hypersensitivity reaction and peripheral neuropathies [319].

Biodegradable polymeric micelles have recently been developed as vehicles for hydrophobic drugs, since these micelles significantly increase the aqueous solubility of hydrophobic drugs and reduce their toxicity by avoiding the use of toxic co-solvents or solubilizing agents [320]. The improved safety profile makes it possible to administer drugs at a higher dose which may enhance their therapeutic efficacy while minimizing

*Adapted with permission. F. Li, Y. Lu, W. Li, D.D. Miller, R.I. Mahato, Synthesis, formulation and in vitro evaluation of a novel microtubule destabilizer, SMART-100, J Control Release, 143 (2010) 151-158. 
their side effects. Previously, we used a poly(ethylene)-b-poly(D,L-lactide) (PEG-PLA) polymeric micelles to solubilize two hydrophobic drugs such as bicalutamide and embelin, and at least 60-fold increase in drug aqueous solubility was observed [321]. Micelles prepared using PEG-PLA copolymer has PLA as a hydrophobic core, which is responsible for drug loading, and PEG as a hydrophilic shell to confer stealth properties and thus prevents recognition by the reticuloendothelial system (RES) [322]. Due to its small size of 20-60 nm and stealth properties, a passive tumor targeting using these micelles through enhanced permeability and retention (EPR) could be achieved [323].

In this study, the anticancer effect of SMART-100 on cancer cells and its ability to circumvent P-gp mediated drug resistance were determined. PEG-PLA micelles were used to improve the aqueous solubility of SMART-100. In addition, the in vitro anticancer activity of SMART-100 loaded micelles and its mechanism of action were determined.

\subsection{Materials and Methods}

\subsubsection{Materials}

Materials Formyl terminated PEG-PLA of 4500-5100 Da was purchased from Polymer Source (Montreal, Canada). HepG2 and C4-2 cells were purchased from American Type Culture Collection (ATCC, Manassas, VA). Parent DU145 and paclitaxel resistant DU145-TXR cells were provided by Professor Evan T. Keller of the University of Michigan. Caspase-Glo ${ }^{\mathrm{TM}} 3$ assay kit was purchased from Promega (Madison, WI). All other reagents were obtained from Sigma-Aldrich (St. Louis, MO).

\subsubsection{Synthesis of SMART-100}

SMART-100, (2-Phenyl-thiazol-4-yl)-(3,4,5-trimethoxy-phenyl)-methanone, was synthesized as described [318]. Briefly, benzonitrile (40 mmol) was mixed with Lcysteine ( $45 \mathrm{mmol}$ ) in $100 \mathrm{~mL}$ of $1: 1 \mathrm{MeOH} / \mathrm{pH} 6.4$ phosphate buffer solution (PBS). The reaction was stirred at $40{ }^{\circ} \mathrm{C}$ for 3 days. The precipitate was removed by filtration, and methanol was removed using a rotary evaporator. $1 \mathrm{M}$ hydrochloric acid was added to the remaining solution to adjust $\mathrm{pH}$ to 2 at $0{ }^{\circ} \mathrm{C}$. The resulting precipitate was filtered to yield a white solid 2-phenyl-4, 5-dihydrothiazole-4-carboxylic acid, which was used directly for the next step.

A mixture of 2-phenyl-4, 5-dihydrothiazole-4-carboxylic acid (5 mmol), 1-ethyl-3-(3'dimethylaminopropyl) carbodiimide (EDCI, $6 \mathrm{mmol}$ ) and hydroxybenzotriazole ( $\mathrm{HOBt}, 5$ $\mathrm{mmol})$ in $\mathrm{CH}_{2} \mathrm{Cl}_{2}(50 \mathrm{~mL})$ was stirred for $10 \mathrm{~min}$. To this solution, $\mathrm{N}$-methylmorpholine (NMM, $11 \mathrm{mmol}$ ) and $\mathrm{HNCH}_{3} \mathrm{OCH}_{3} \mathrm{HCl}$ salt $(5 \mathrm{mmol})$ were added and stirring continued at room temperature for 6-8 $\mathrm{h}$. The reaction mixture was diluted with $\mathrm{CH}_{2} \mathrm{Cl}_{2}$ $(100 \mathrm{~mL})$ and sequentially washed with water, satd. $\mathrm{NaHCO}_{3}$, brine and dried over 
$\mathrm{MgSO}_{4}$. The solvent was removed under reduced pressure to yield a crude product, which was purified by column chromatography to get 2-phenyl-4, 5-dihydrothiazole-4carboxylic acid methoxymethylamide (Yield 92.0\%). A solution of 2-phenyl-4, 5dihydrothiazole-4-carboxylic acid methoxymethylamide (1 equiv) in $\mathrm{CH}_{2} \mathrm{Cl}_{2}$ was cooled to $0{ }^{\circ} \mathrm{C}$ and distilled DBU ( 2 equiv) was added. Bromotrichloromethane (1.7 equiv) was then introduced dropwise via syringe over $10 \mathrm{~min}$. The reaction mixtures were allowed to warm to room temperature and stirred overnight. Upon washing with satd. aqueous $\mathrm{NH}_{4} \mathrm{Cl}(2 \times 50 \mathrm{~mL})$, the aqueous phase was extracted with ethyl acetate $(3 \times 50 \mathrm{~mL})$. The combined organic layers were dried on $\mathrm{MgSO}_{4}$, filtered and concentrated in vacuo. The residue was purified by flash chromatography as needed providing 2-phenyl-thiazole-4carboxylic acid methoxymethylamide (87.1\%).

To a solution of 3, 4, 5-trimethoxyphenylmagnesium bromide $(0.5 \mathrm{M}, 3 \mathrm{~mL})$ in $2 \mathrm{~mL}$ tetrahydrofuran (THF) was charged a solution of 2-phenyl-thiazole-4-carboxylic acid methoxymethylamide $(1 \mathrm{mmol})$ in $3 \mathrm{ml} \mathrm{THF}$ at $0{ }^{\circ} \mathrm{C}$. The mixtures were stirred for 30 min until amides disappeared on TLC plates. The reaction mixture was quenched with satd. $\mathrm{NH}_{4} \mathrm{Cl}$, extracted with ethyl ether, dried with $\mathrm{MgSO}_{4}$. The solvent was removed under reduced pressure to yield a crude product, which was purified by column chromatography to obtain pure compound SMART-100.

\subsubsection{Preparation and characterization micelles}

\subsubsection{Preparation of micelles}

Drug loaded micelles were prepared by a film-dispersion method. Briefly, various amounts of SMART-100 and PEG-PLA were dissolved in acetonitrile and a thin film was formed after removing solvent under reduced pressure. The resulting film was hydrated and sonicated. The resultant formulation was then centrifuged to remove residual free drug. The supernatant was then filtered using a $0.22 \mu \mathrm{m}$ filter and used within $48 \mathrm{~h}$.

\subsubsection{Determination of drug solubility and loading efficiency}

To determine water solubility of free drug, $1 \mathrm{mg}$ of SMART-100 was suspended in $1 \mathrm{~mL}$ water and shake for $48 \mathrm{~h}$ at room temperature. The suspension was centrifuged at 10,000 rpm for $10 \mathrm{~min}$ and filtered on $0.22 \mu \mathrm{m}$ filter. To determine the solubility of SMART-100 in micelles, $50 \mu \mathrm{L}$ of SMART-100 loaded micelles solution was diluted with acetonitrile, and the drug concentration was measured by UV-spectrometer or reverse phase high performance liquid chromatography (RP-HPLC, Waters, Milford, MA) with UV detector at $292 \mathrm{~nm}$ using a reverse phase C18 column (250 mm x $4.6 \mathrm{~mm}$, Alltech, Deerfield, IL). The mobile phase was composed of 40:60 V/V water and acetonitrile. SMART-100 concentration was calculated by peak area according to the following calibration equation: $\mathrm{C}=188.24 \mathrm{~A}-593.64\left(\mathrm{R}^{2}=1\right.$, detection limit: $\left.2 \mathrm{ng} / \mathrm{mL}\right)$. The drug encapsulation efficiency of SMART-100 in polymeric micelles was calculated with the following equation: drug encapsulation efficiency $\%=(($ weight of drug in micelles) $/$ (weight of drug origally fed) $) \times 100 \%$. 
Fold of drug solubility increase were calculated with following equation: fold of drug solubility increse $=$ drug solubility in micelles / drug solubility in water.

\subsubsection{Measurement of particle size}

The particle size distribution of micelles was determined by dynamic light scattering with Malvern Nano ZS. The intensity of scattered light was detected at $90^{\circ}$.

\subsubsection{Propidium iodide staining and cell cycle analysis}

Cells were cultured in a 24-well plate to $90 \%$ confluence and treated with SMART-100 $(2.8 \mu \mathrm{M})$ for $24 \mathrm{~h}$. Cells were trypsinized and fixed in $70 \%$ ice-cold ethanol. After washing with PBS, cell pellet was re-suspended in $5 \mu \mathrm{g} / \mathrm{mL}$ propidium iodide staining solution for $15 \mathrm{~min}$ at room temperature. Cell cycle distribution was measured by flow cytometry (Becton, Dickinson, NJ). Results from 10,000 fluorescent events were obtained for analysis.

\subsubsection{MTT assay}

Cells were seeded in 96-well plates at a density of 5,000 cells per well. At the end of treatment, cell culture media was replaced by $100 \mu \mathrm{l}$ medium with $0.5 \mathrm{mg} / \mathrm{mL}$ MTT (3(4,5-dimethyl-thiazol-2-yl)-2, 5-diphenyl tetrazolium bromide and incubated for $1 \mathrm{~h}$ at $37^{\circ} \mathrm{C}$. After removing the media, $200 \mu \mathrm{L}$ of DMSO was added into each well to dissolve the formazan crystals. The absorbance was measured in a microplate reader at a wavelength of $560 \mathrm{~nm}$. Cell viability was expressed as the percentage of control group.

\subsubsection{Calcein acetoxymethylester (calcein AM) assay}

Cells were seeded into a black wall clear bottom 96-well plate at a density of 20,000 cells per well one day before the experiment. After treating cells with various concentration of SMART-100 in $50 \mu \mathrm{L}$ DPBS for $20 \mathrm{~min}$ at $37^{\circ} \mathrm{C}, 50 \mu \mathrm{L}$ Calceim AM $(10 \mu \mathrm{M})$ in DPBS were added to each well and the plate was incubated at $37^{\circ} \mathrm{C}$ for additional $20 \mathrm{~min}$. Fluorescence intensity in each well were determined using a SpectraMax M2/M2e spectrofluorometer (Sunnyvale, CA) at the excitation wavelength of $494 \mathrm{~nm}$ and emission wavelength of $517 \mathrm{~nm}$.

\subsubsection{Determination of caspase 3 activity}

Cells were seeded in a 48 -well plate to reach $80 \%$ confluence before experiment. After treating the cells for $16 \mathrm{~h}$, culture media was removed and $100 \mu \mathrm{L}$ Caspase Glo 3 reagent was added to each well. After gently mixing the content in each well, the plate was 
incubated at room temperature for 60 min protected from light. Finally, $70 \mu \mathrm{L}$ of the reaction solution was measured using a luminometer.

\subsubsection{Establishing a luciferase expression cell line for IVIS imaging}

For stable luciferase expression, C4-2 cells were seeded in a 6-well plate $24 \mathrm{~h}$ before being transfected with pRc/CMV2-Luc plasmid (from Dr. Shu Wang, National University of Singapore) for $18 \mathrm{~h}$. Two days after transfection, cells were transferred into a T-25 cell culture flask and selected with $600 \mu \mathrm{g} / \mathrm{mL}$ G418. The resulting bioluminescent cells were maintained in media containing $300 \mu \mathrm{g} / \mathrm{mL}$ G418. These cells were then seeded from 120,000 to 100 cells into a black wall clear bottom 96-well plates. D-luciferin $(150 \mu \mathrm{g} / \mathrm{mL})$ in DPBS was added to each well at 2 min prior to imaging using an IVIS imaging system (Xenogen, Alameda, CA).

\subsection{Results}

\subsubsection{Synthesis and characterization of SMART-100}

SMART-100 was synthesized as described by Lu et al.[318], and illustrated in Figure 5.1A. SMART-100 was characterized by ${ }^{1} \mathrm{H}$ NMR (Figure 5.1B), ${ }^{13} \mathrm{C}$ NMR and ESI-MS. ${ }^{1} \mathrm{H}$ NMR $\left(300 \mathrm{MHz}, \mathrm{CDCl}_{3}\right): \delta 8.29$ (s, $\left.1 \mathrm{H}\right), 8.03$ (q, $\left.2 \mathrm{H}\right), 7.80(\mathrm{~s}, 2 \mathrm{H}), 7.49-7.47$ (m, 3 $\mathrm{H}), 3.96(\mathrm{~s}, 6 \mathrm{H}), 3.97$ (s, $3 \mathrm{H}) .{ }^{13} \mathrm{C} \mathrm{NMR}\left(75 \mathrm{MHz}, \mathrm{CDCl}_{3}\right)$ : 185.1, 168.1, 167.0, 155.4, 152.7, 133.1, 131.9, 130.6, 129.1, 127.7, 126.6, 108.6, 60.9, 56.2. MS (ESI) m/z 378.1 $[\mathrm{M}+\mathrm{Na}]^{+}$.

\subsubsection{Anti-cancer activity of SMART-100 on HepG2 cells}

The anticancer activity of SMART-100 was determined in HepG2 cells with MTT assay. SMART-100 effectively inhibited HepG2 cell growth (Figure 5.2), with an $\mathrm{IC}_{50}$ of around $0.56 \mu \mathrm{M}$. The effect was observed as early as $24 \mathrm{~h}$ post treatment and became more significant after $48 \mathrm{~h}$ post treatment. There is no significant difference between " 0 " group, which is the cells treated with $0.5 \%$ DMSO and "DMEM" group, which is the cells treated with DMEM cell culture medium. Dose response curve was of typical sigmoid shape, with steep linear response at the mid-range dose groups. While there was no measurable effect at low doses $(\leq 0.06 \mathrm{nM})$, the dose response curve became less steep and finally reaches plateau at high dose groups.

\subsubsection{Anti-cancer activity of SMART-100 in resistant prostate cancer cells}

We also tested the anticancer effect of SMART-100 on DU145-TXR cell, a paclitaxel resistant prostate cancer cell line with high P-gp activity and the parent DU145 cell, a 

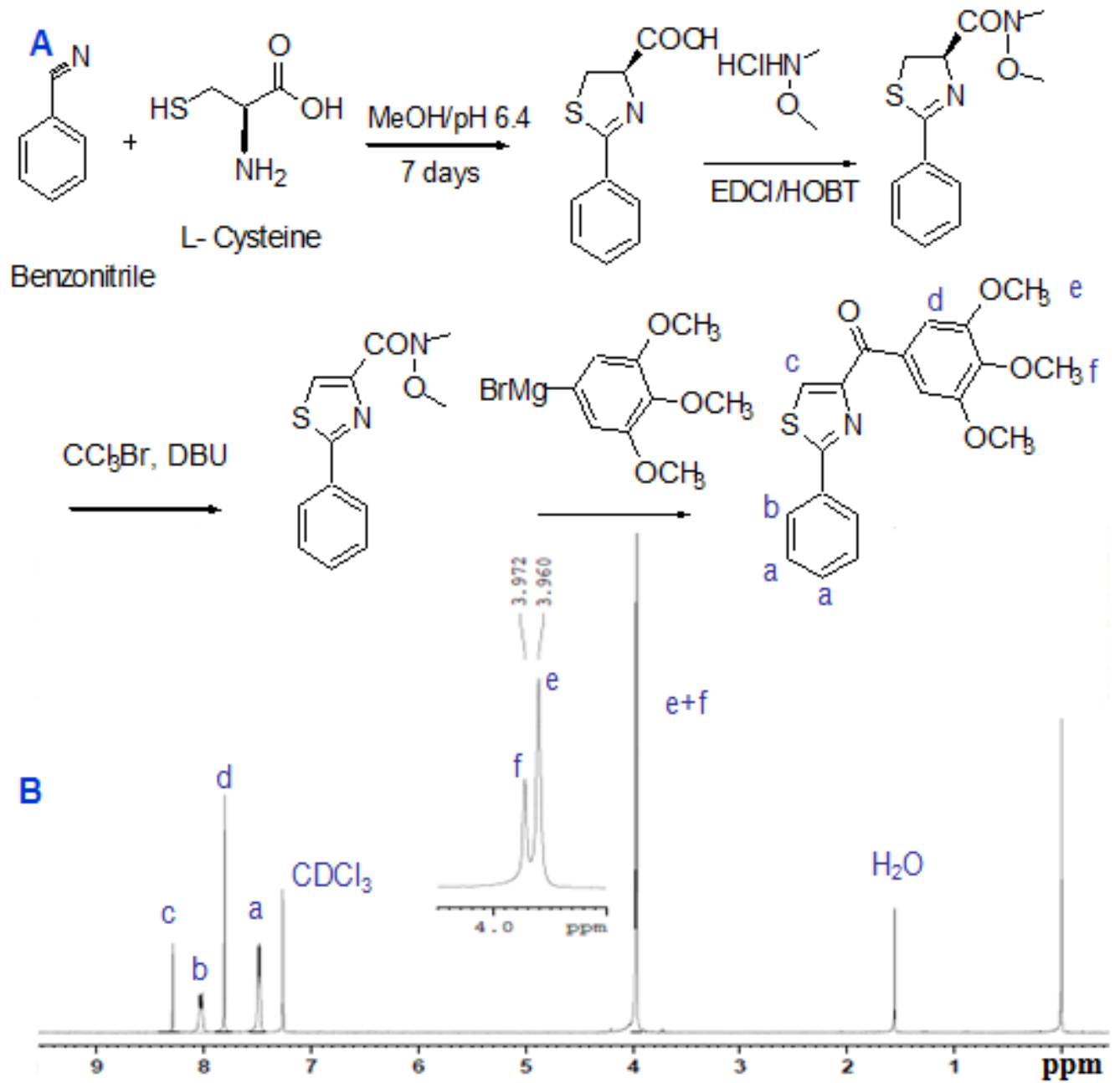

Figure 5.1 Synthesis and characterization of SMART-100.

(A) Synthesis scheme of SMART-100. (B) ${ }^{1}$ H NMR spectrum of SMART-100. 


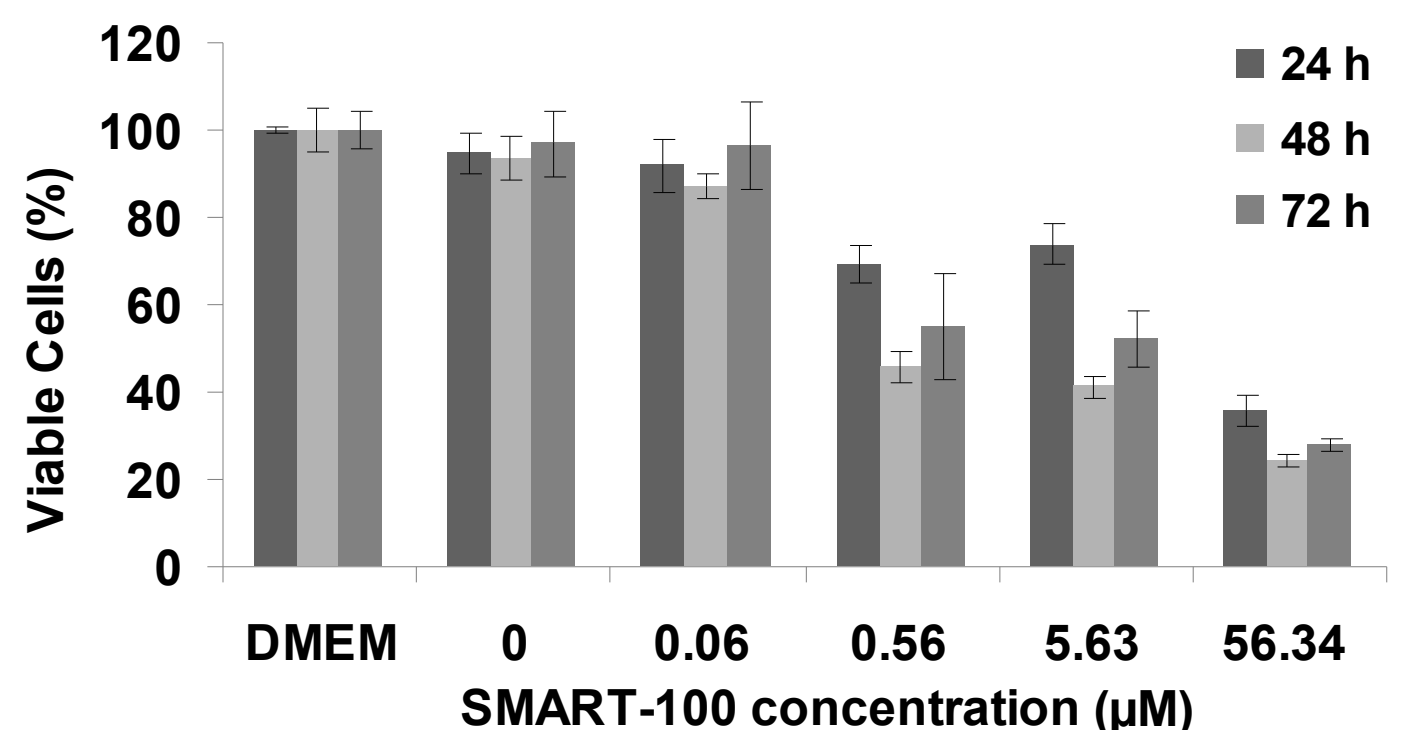

Figure 5.2 Anti-cancer effect of SMART-100 on HepG2 cells.

HepG2 cells were treated with increasing concentration of SMART-100 for 24, 48 and 72 h. Cell viability was determined by MTT Assay and expressed as $\%$ of control. 
paclitaxel sensitive cell with low P-gp activity. Even though paclitaxel showed good activity on DU145 cells $\left(\mathrm{IC}_{50}<50 \mathrm{nM}\right)$, its efficacy was greatly reduced on DU145-TXR cells and killing only $20 \%$ of cells at a concentration of $1 \mu \mathrm{M}$ (Figure 5.3A). In contrast, SMART-100 showed equally good anticancer activity on both DU145 and DU145-TXR cells with $\mathrm{IC}_{50}$ around $70 \mathrm{nM}$ (Figure 5.3B). From Figure 5.3B, we could see that SMART-100 at $1 \mu \mathrm{M}$ inhibited $63.7 \%$ and $67.6 \%$ cancer cell growth for DU145 and DU145-TXR, respectively. 74.2\% (DU145) and 74.9\% (DU145-TXR) cancer cell growth inhibition was achieved by SMART-100 at concentration of $5.63 \mu \mathrm{M}$. 82.1\% (DU145) and $83.8 \%$ (DU145-TXR) cancer cell growth inhibition was achieved by SMART-100 at concentration of $11.26 \mu \mathrm{M}$.

Intracellular calcein levels in DU145 cells were much higher than those in DU145-TXR cells (Figure 5.4A), indicating higher P-gp activities in DU145-TXR cells but not in DU145 cells. The treatment of verapamil, a P-gp inhibitor, significantly increased intracellular calcein levels in DU145-TXR cells in a dose dependent manner, while no changes in intracellular calcein levels were observed in DU145 cells. Treatment of DU145-TXR cells with SMART-100 also increased intracellular calcein levels, while treatment of paclitaxel showed no effect (Figure 5.4B). This indicated that SMART-100 might also act as a P-gp inhibitor in addition to its anti-mitotic activity and thus overcome the drug resistance in DU145-TXR cells due to P-gp over-expression.

\subsubsection{Micellar solubility of SMART-100}

Although SMART-100 showed good anticancer activity, its application was limited due to its poor water solubility, which is less than $2 \mathrm{ng} / \mathrm{mL}$. Therefore, PEG-PLA micelles were used to improve its solubility. A film dispersion method was used to prepare micelles [321]. The mean particle size of micelles was around $50 \mathrm{~nm}$ and no significant difference in particle size was observed in micelles with and without drug. These micelles were stable for at least 12 days without significant change in particle size.

When we increased the theoretical loading from $0.5 \%$ to $5 \% \mathrm{w} / \mathrm{w}$, the drug solubility increased from $26.8 \pm 0.1 \mu \mathrm{g} / \mathrm{ml}$ to $48.0 \pm 0.3 \mu \mathrm{g} / \mathrm{ml}$ until theoretical loading reached $1 \%$ (Figure 5.5A). The drug loading efficiency decreased significantly after the theoretical loading became larger than 1\% (Figure 5.5B).To further increase the drug solubility, the effect of polymer concentration on drug solubility was determined. The solubility of SMART-100 increased linearly from $48.0 \pm 0.3$ to $213.9 \pm 8.1 \mu \mathrm{g} / \mathrm{mL}\left(2.4 \times 10^{4}\right.$ to $1.1 \mathrm{x}$ $10^{5}$ folds as that of free SMART-100 in water), when the polymer concentration was increased from $5 \mathrm{mg} / \mathrm{mL}$ to $20 \mathrm{mg} / \mathrm{mL}$ (Figure $5.5 \mathrm{C}$ ). The drug loading efficiency was around $100 \%$ for all tested polymer concentration (Figure 5.5D).

\subsubsection{Anticancer effect of SMART-100 loaded PEG-PLA micelles}

No obvious cytotoxicity was observed in blank PEG-PLA micelles group with a polymer concentration of as high as $1 \mathrm{mg} / \mathrm{mL}$ (Figure 5.6). Significant anticancer effect was 

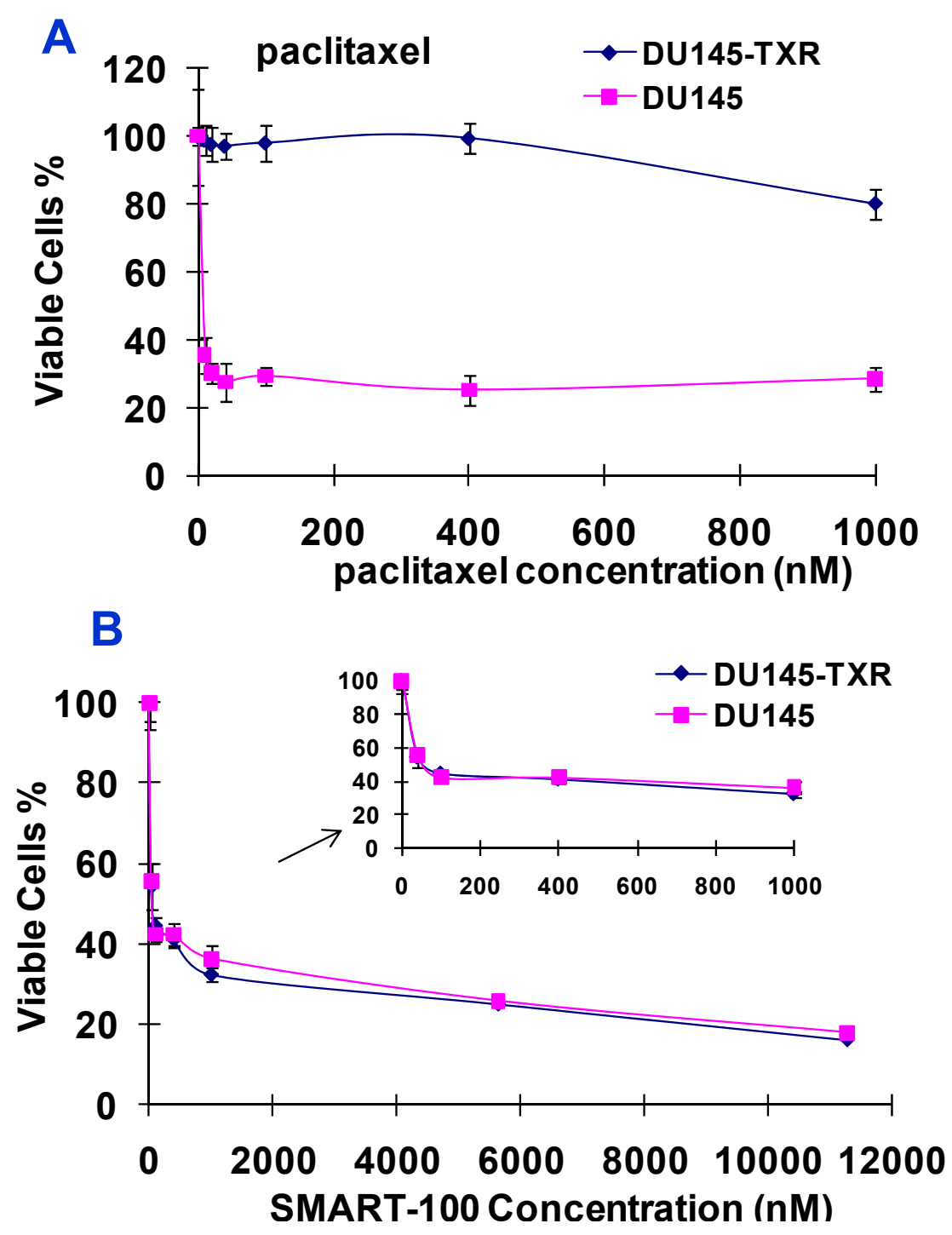

Figure 5.3 Anti-cancer effect of SMART-100 on MDR cancer cells.

Inhibition of DU145 and DU145-TXR cells growth by SMART-100 (A) and paclitaxel (B) Cell viability was determined by MTT Assay $48 \mathrm{~h}$ after drug treatment and expressed as the $\%$ of control. 
A
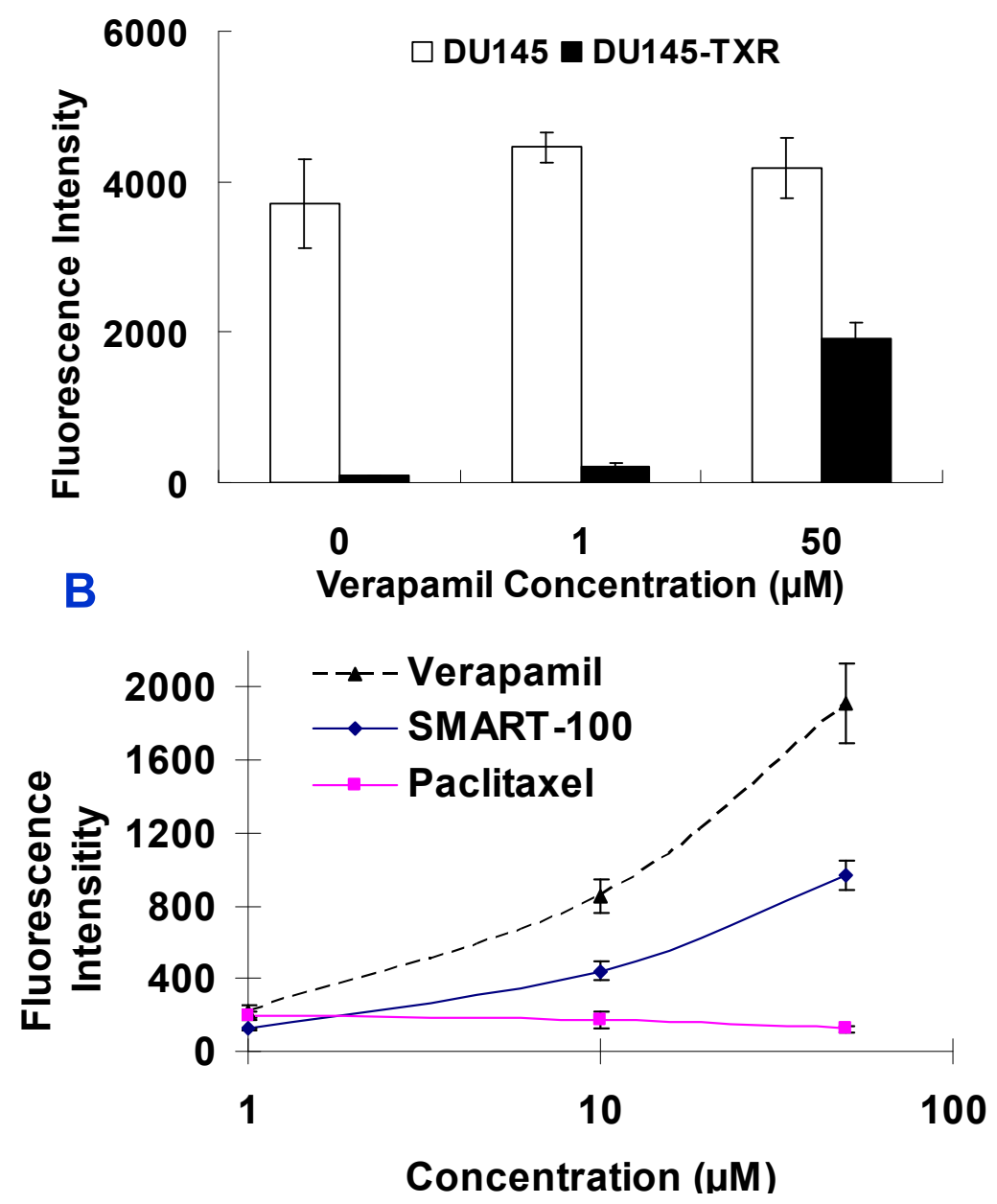

Figure 5.4 Effect of SMART-100 on P-gp activity.

(A) Intracellular calcein accumulation in DU145 and DU145-TXR cells. (B) Effect of SMART-100 on intracellular calcein accumulation in DU145-TXR cells. Results are expressed as the mean $\pm \mathrm{SE}(\mathrm{n}=3)$. 

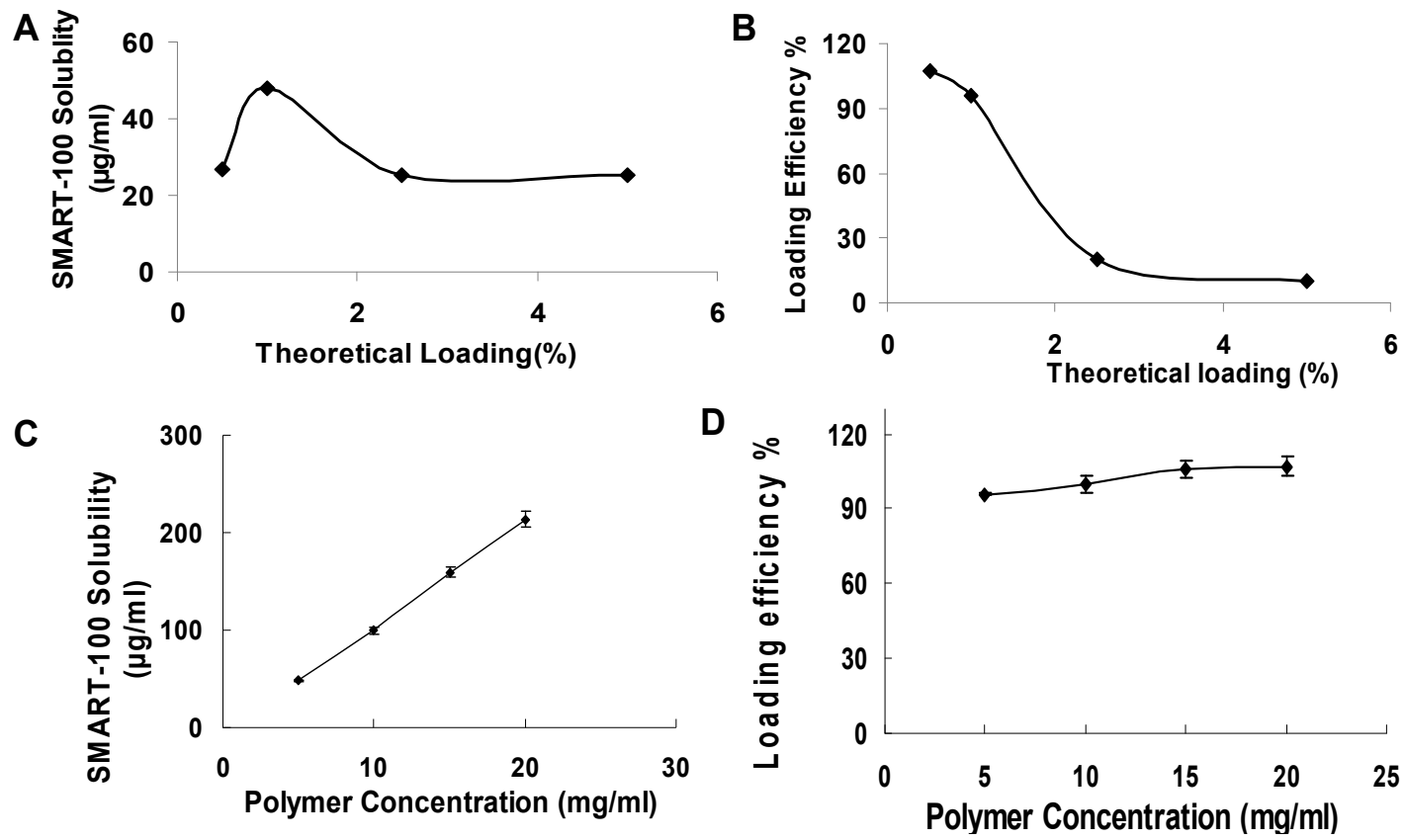

Figure 5.5 Solubilization of SMART-100 with PEG-PLA.

Effect of theoretical loading on SMART-100 solubility (A) and loading efficiency (B); Effect of polymer concentration on SMART-100 solubility (C) and loading efficiency (D). Results are expressed as the mean $\pm \operatorname{SE}(n=3)$. 


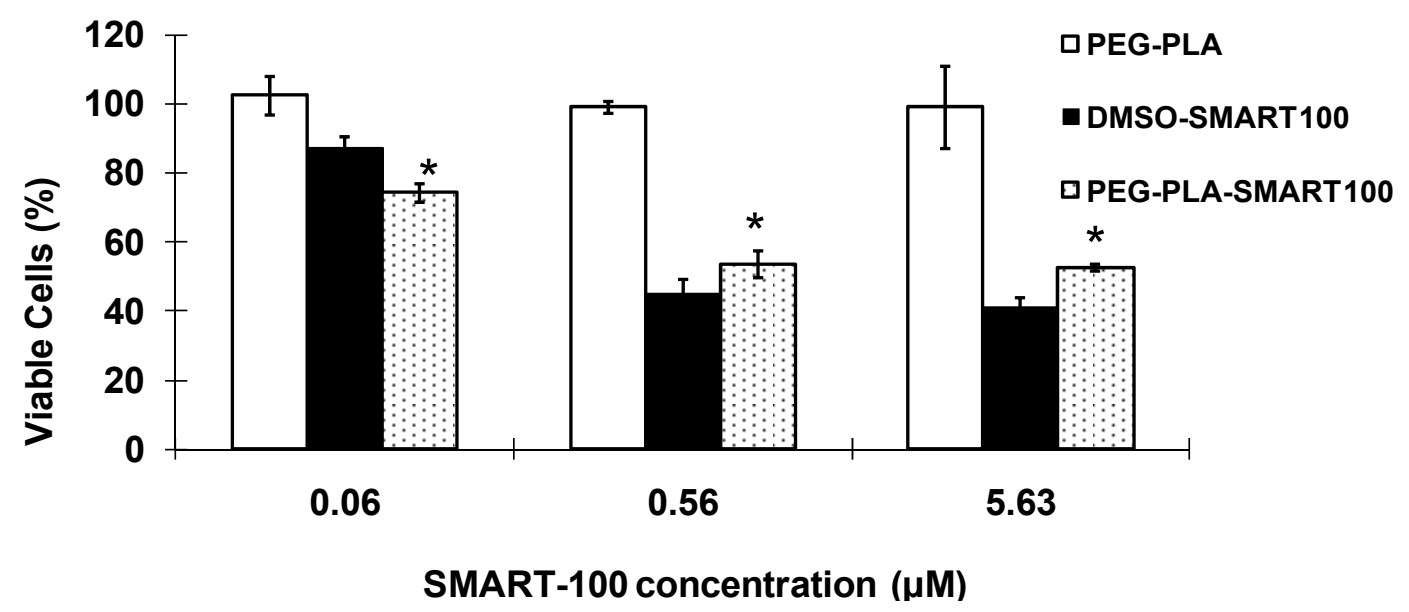

Figure 5.6 Anti-cancer effect of PEG-PLA micelles formulated SMART-100.

HepG2 cells were treated with different SMART-100 formulations for $48 \mathrm{~h}$. Cell viability was determined by MTT Assay and expressed as the percentage of control. *, p $<0.001$, compared with blank PEG-PLA control. 
observed in both SMART-100 loaded PEG-PLA micelles and SMART-100 dissolved in DMSO and the anticancer activity increased with the dose. Even though the final DMSO concentration used was $0.5 \%$, which did not show any obvious toxicity on our experiment, no direct comparison can be made with the results of SMART-100 dissolved in DMSO, as DMSO is known to kill tumor and may not be suitable for in vivo applications.

\subsubsection{Cell cycle perturbation}

The treatment of SMART-100 formulated in both DMSO and PEG-PLA micelles caused cell cycle arrest in HepG2 cells, while no effect was observed after treating cells with blank PEG-PLA micelles or DMSO (Figure 5.7). The treatment of SMART-100 formulations caused the cells in $\mathrm{G} 0 / \mathrm{G} 1$ phase decreased from $49.18 \pm 1.59 \%$ in the control group to $21.03 \pm 0.54 \%$ in DMSO dissolved SMART-100 group and $19.24 \pm$ $0.89 \%$ in PEG-PLA micelles formulated SMART-100 group, respectively. While the cells in $\mathrm{G} 2 / \mathrm{M}$ phase were increased from $29.59 \pm 1.55 \%$ in control group to $73.06 \pm$ $1.68 \%$ in DMSO dissolved SMART-100 group and $74.91 \pm 1.10 \%$ in PEG-PLA micelles formulated SMART-100 group, respectively. In addition, we also determined the effect of treatment time on cell cycle distribution (Figure 5.7B). Cells were treated with SMART-100 loaded PEG-PLA micelles for 24, 48, and $72 \mathrm{~h}$. There was no change in the percentage of cells in G0/G1 phase after treating the cells for prolonged time at 48 or 72 $\mathrm{h}$. The percentage of the cells in G2/M phase was decreased from $74.91 \pm 1.1 \%$ at $24 \mathrm{~h}$ to $68.71 \pm 1.95 \%$ at $48 \mathrm{~h}$ and to $65.5 \pm 1.65 \%$ at $72 \mathrm{~h}$. In addition, the cells in sub G1 phase were also increased from $0.29 \pm 0.21 \%$ at $24 \mathrm{~h}$ to $2.96 \pm 1.34 \%$ at $48 \mathrm{~h}$ and to $8.94 \pm$ $1.15 \%$ at $72 \mathrm{~h}$. The increase of cell population in subG1 phase indicated that apoptotic cells were increased after SMART-100 treatment for prolonged time.

\subsubsection{Caspase 3 activity}

In the cell cycle analysis, we found that the treatment of SMART-100 resulted in increased cell population in subG1 phase, which is an indication of apoptosis. To confirm whether cell death induced by SMART-100 was associated with apoptosis, caspase 3 activity was measured after treating HepG2 cells with SMART-100. As shown in Figure 5.8, no significant difference was observed between untreated cell and cells treated with DMSO or empty PEG-PLA micelles. The treatment of SMART-100 with both DMSO and PEG-PLA micelles at a concentration of $2.8 \mu \mathrm{M}$ resulted in increased caspase 3 activity $(\mathrm{p}<0.05)$, which is comparable to those caused by paclitaxel with the same molar concentration $(\mathrm{p}>0.05)$. This result indicated that SMART-100 may cause cell apoptosis by the activation of caspase 3 activity. However, increase of caspase 3 activity in SMART-100 treated groups was relatively low compared with cells treated with staurosporine, a well-known caspase 3 activator $(\mathrm{p}<0.01)$. 

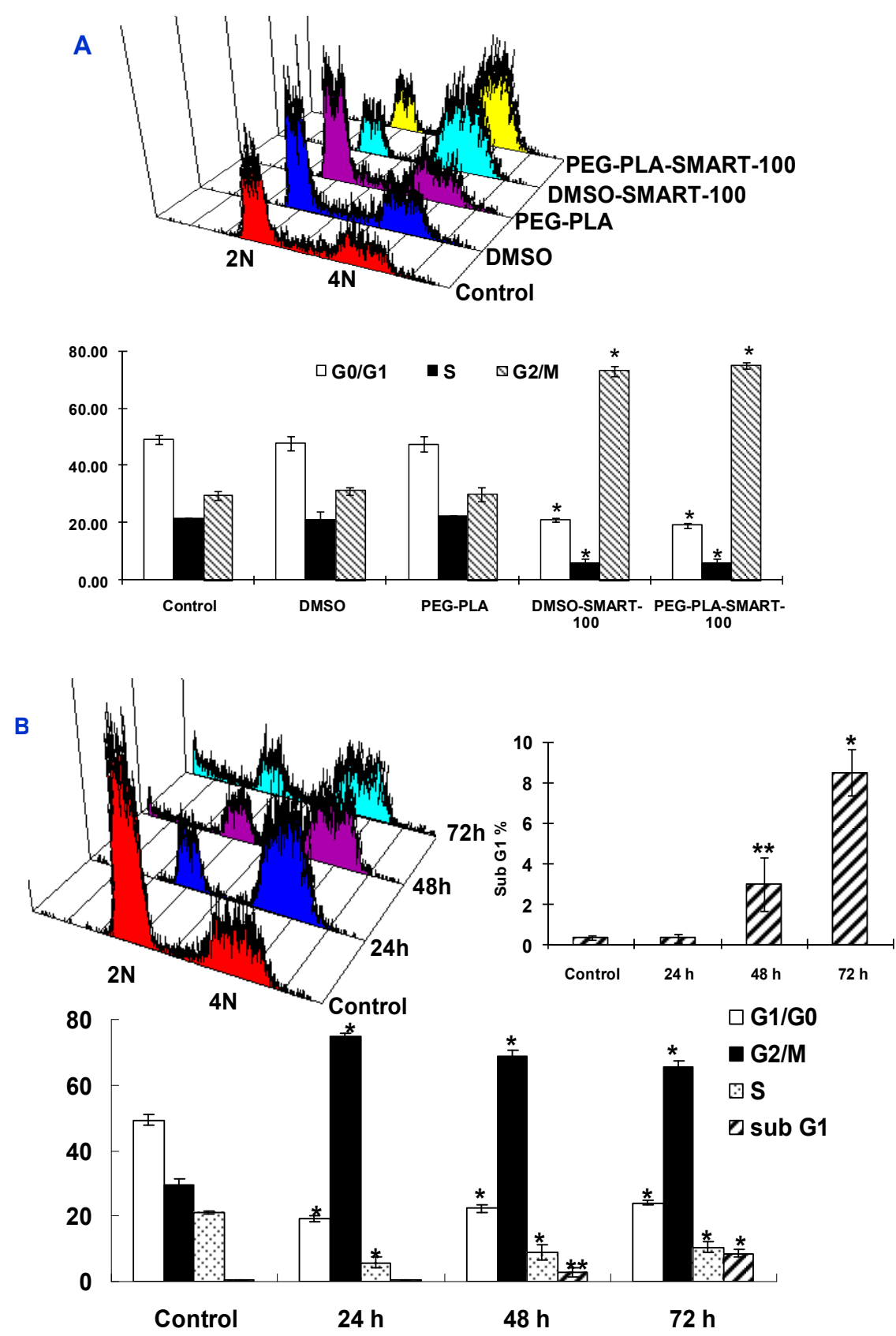

Figure 5.7 Effect of SMART-100 on cell cycle and apoptosis.

(A) HepG2 cells were treated with different SMART-100 formulations (drug concentration $1 \mu \mathrm{g} / \mathrm{ml}$ ) for $24 \mathrm{~h}$ and cell cycles were analyzed by flow cytometry. Upper panel, Cell cycle distribution; Lower panel, quantitative analysis. (B) Time course of cell cycle perturbation in HepG2 cells treated with SMART-100 loaded PEG-PLA micelles. Upper left panel, cell cycle distribution; Upper right panel, graphical representation of apoptotic index (percent sub-G1 cells) over time; and Lower panel, quantitative analysis. Results are expressed as the mean $\pm \mathrm{SE}(\mathrm{n}=3)$. ${ }^{*} \mathrm{P}<0.001,{ }^{* *} \mathrm{P}<0.01$, compared with control. 


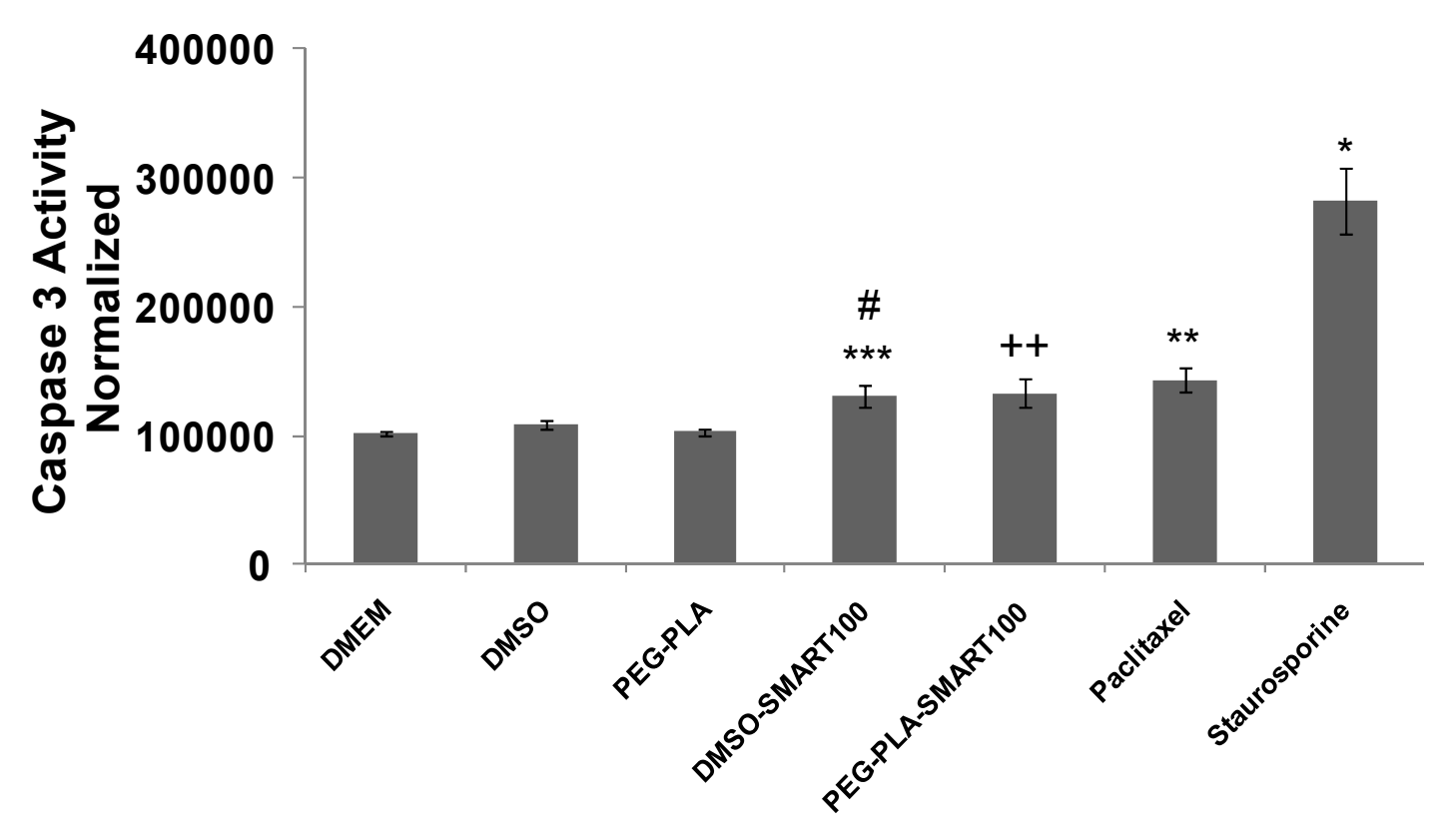

Figure 5.8 Effect of SMART-100 on caspase 3 activity.

After treatment with $1 \mu \mathrm{g} / \mathrm{ml} \mathrm{SMART-100} \mathrm{or} \mathrm{same} \mathrm{molar} \mathrm{concentration} \mathrm{of} \mathrm{paclitaxel} \mathrm{or}$ saturosporeine for $16 \mathrm{~h}$, caspase 3 activity was determined. Caspase 3 activities were expressed as relative light unit (RLU) and normalized by cell viability. Drugs were dissolved in DMSO, unless otherwise stated. Results are the mean $\pm \operatorname{SE}(n=3)$. ${ }^{*}, \mathrm{p}<$ $0.001,{ }^{* *}, \mathrm{p}<0.01,{ }^{* * *}, \mathrm{p}<0.05$ compared with DMSO control. ++, $\mathrm{p}<0.01$ compared with blank PEG-PLA micelles. \#, $\mathrm{p}<0.001$, compared with saturosporeine. 


\subsubsection{IVIS imaging for assessing antitumor activity}

We established a luciferase expressing C4-2 prostate cancer cell line for IVIS imaging (Figure 5.9A). There was a good correlation between the cells number and bioluminescence signals captured by IVIS imaging $\left(R^{2}=0.9769\right.$, Figure 5.9B), thus live cell number could be quantitatively determined by IVIS imaging. Treatment with SMART-100 resulted in significant reduction in the bioluminescence signal (Figure 5.9C). However, the treatment with empty PEG-PLA micelles did not cause any change in bioluminescence signal. The viable cell number was also been quantitatively determined by measuring bioluminescence signal (Figure 5.9D).

\subsection{Discussion}

The discovery of paclitaxel has resulted in significant progress for the treatment of several cancers including breast, ovarian, and non-small cell lung cancers. Paclitaxel works through stabilizing microtubules, and thus causing mitotic arrest and apoptosis. Despite the potent anticancer activity of paclitaxel, its clinical application is often undermined due to the occurrence of MDR. Cancer cells become resistant to anticancer drugs due to the change in cell membrane ATP-binding cassette (ABC) super family transporters such as P-gp, multiple drug resistance protein (MRP) and breast cancer resistance protein (ABCG2). Particularly, the over-expression of P-gp encoded by MDR1 gene is widely investigated and known to be responsible for the resistance to several anticancer agents such as paclitaxel and docetaxel.

Several strategies have been studied to overcome MDR mechanism including the use of novel drug delivery systems, co-administration of P-gp inhibitors, and the search of new compounds that could overcome MDR. Drug delivery systems such as HPMA copolymer drug conjugates [324], polymeric micelles [325], liposomes [326], and nanoparticles [327] have been successfully used to reduce the drug resistance by enhanced intracellular drug delivery and/or inhibition of P-gp activities. In addition to formulation approaches, scientists have also successfully discovered several new compounds to overcome MDR. Ixabepilone, for example, has been approved by the FDA as a drug for MDR breast cancer [328].

To test whether SMART-100 could overcome MDR, a paclitaxel-resistant cell line, DU145-TXR, was used in this study. DU145-TXR is resistant to paclitaxel as well as several other anticancer agents including estramustine phosphate, docetaxel, doxorubicin and vinblastine [329]. SMART-100 showed potent anticancer activities in both paclitaxel-sensitive (DU145) and paclitaxel-resistant (DU145-TXR) cancer cells (Figure 5.3B). These results suggest that SMART-100 has the potential to be used for treating MDR cancers.

To further understand why SMART-100 could keep its anticancer activity in paclitaxelresistant cells capable of overexpressing P-gp, Calcein acetoxymethylester (Calcein AM) assay was carried out to determine the effect of SMART-100 on P-gp functions. Calcein 

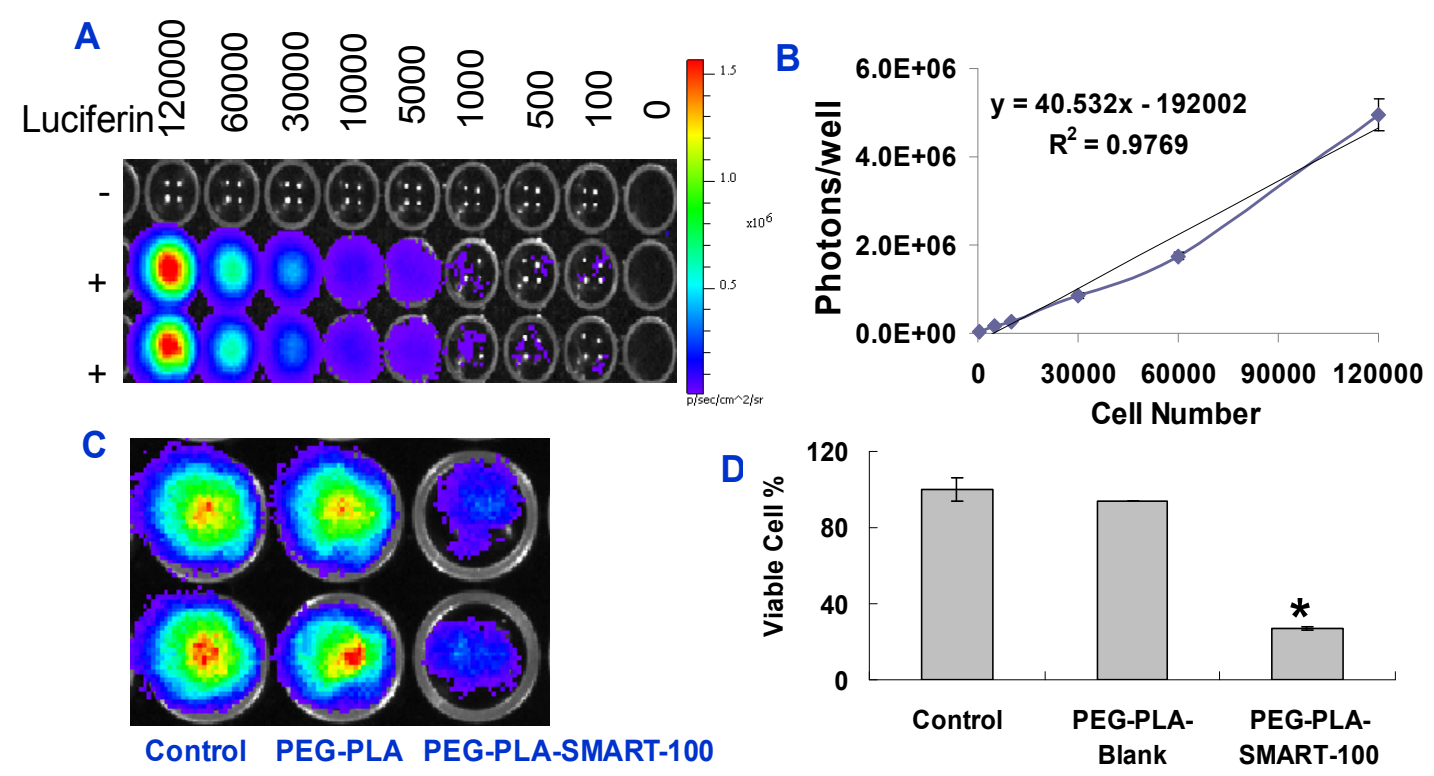

Figure 5.9 Determination of anti-cancer activity by IVIS imaging.

(A) In vitro bioluminescence of C4-2 human prostate tumor cell lines expressing luciferase. C4-2-luc cells were seeded from 120,000 to 100 cells per well and imaged after the addition of luciferin. Wells containing cells but no luciferin were served as negative controls. (B) Correlation between cell number and mean bioluminescence signal. (C) Effect of SMART-100 loaded PEG-PLA micelles on the Inhibition of C4-2Luc cell growth. Cells were treated with blank PEG-PLA micelles and SMART-100 loaded micelles for 4 days at the dose of $1 \mu \mathrm{M}$ and the in vitro bioluminescence was determined by IVIS imaging. (D) The cell viability was quantitatively determined by bioluminescence reading and expressed as percentage of control group. ${ }^{*}, \mathrm{p}<0.001$, compared with blank PEG-PLA control. 
AM is a substrate for efflux activity of P-gp. In cells with high P-gp activity, Calcein AM is constantly pumped out, preventing intracellular accumulation of fluorescent calcein. The inhibition of P-gp function by inhibitors such as verapamil results in reduced efflux of fluorescent calcein and thus increases intracellular fluorescence intensity. The change of fluorescence intensity could be quantitatively determined by fluorometer and used as an indicator for P-gp activities. The treatment of SMART-100 significantly increased the intracellular accumulation of calcein, which indicated that it might be an inhibitor of P-gp and prevented the efflux of drug out of P-gp over-expressing cells (Figure 5.4). Therefore, inhibition of P-gp activity might be a mechanism of SMART-100 to overcome multiple drug resistance.

Our previous study showed that SMART-100 works by targeting and destabilizing microtubules. As a microtubule destabilizer, its anti-proliferative effect is involved in the inhibition of tubulin polymerization [318]. The cell cycle analysis indicated that after treatment with SMART-100 the cells were arrested in G2/M phase (Figure 5.7). Since the destabilization of microtubule dynamics will result in the cell cycle arrest at mitotic phase, cell cycle analysis experiment further confirmed that the anticancer mechanism of SMART-100 is through destabilizing microtubule. We also found that after SMART-100 treatment cells in sub-G1 phase were significantly increased (Figure 5.7). The increase in cell population in sub-G1 phase indicated the induction of cell apoptosis by SMART-100 $[330,331]$. The increase in caspase 3 activity is an important marker for cell apoptosis. Treatment with SMART-100 increased caspase 3 activities in this study (Figure 5.8), which indicated the occurrence of apoptosis. From the binding of microtubule destabilizer with the tubulin to apoptosis is a complicated intracellular process, which involves multiple signal pathways, including the checkpoint of mitotic spindle assembly, the activation of cyclin-dependent kinases, and the JNK/SAPK [332]. However, these signal pathways are beyond the scope of this study.

PEG-PLA micelles have been successfully used as a Cremophor EL free formulation for paclitaxel (Genexol-PM), which is under multicenter phase II clinical trial [333]. Because of its superior performance as a delivery system for paclitaxel, we used PEG-PLA micelles to improve the solubility of SMART-100. Due to the high $\log \mathrm{P}$ value (4.08) of SMART-100, its aqueous solubility is very low (below the detection limit, $2 \mathrm{ng} / \mathrm{mL}$ ). However, the use of PEG-PLA micelles significantly increased its aqueous solubility. The maximum solubility reached to $213.9 \pm 8.1 \mu \mathrm{g} / \mathrm{mL}$, where SMART-100 solubility was increased by at least $1.1 \times 10^{5}$ folds (Figure 5.5). Polymeric micelles are known to enhance the solubilization of hydrophobic compounds by accommodating them in the hydrophobic core of polymers. A critical parameters involves in the solubilization process is polymer-drug compatibility, which could be evaluated by solubility difference $(\Delta)$ and Flory-Huggins interaction parameter $\left(\mathrm{X}_{\mathrm{sp}}\right)$ [334]. We have calculated the solubility parameters of SMART-100 and PLA hydrophobic core by Molecular Modeling Pro Software (ChemSW Inc., Fairfield, CA). The calculated parameters for PLA is 19.7 $(\delta \mathrm{d}), 2.48(\delta \mathrm{p})$ and $20.9(\delta \mathrm{h})$, while those of SMART-100 is $20.3(\delta \mathrm{d}), 6.5(\delta \mathrm{p})$ and 9.2 $(\delta \mathrm{h})$. Thus, the solubility difference $(\Delta)$ between PLA and SMART-100 is 12.4. Typically, a solubility difference value less than 5 is needed to observed good solubility [335]. 
Therefore, we plan to design and modify the hydrophobic core of the polymer to make it more compatible with SMART-100 and further increase the solubility of SMART-100. 


\section{CHAPTER 6. SYNTHESIS AND CHARACTERIZATION OF AMPHIPHILIC LIPOPOLYMERS FOR MICELLAR DRUG DELIVERY*}

\subsection{Introduction}

Many anticancer agents are poorly soluble in water, which can cause serious problems for their clinical application. Dimethyl sulfoxide (DMSO), Ctenophore EL, Tween 80 or other surfactants are commonly used to solubilize these drugs. However, these solubilizing agents are harmful to the liver and kidney and cause dose-dependent hemolysis and acute hypersensitivity reactions [319, 336]. In addition, because of high critical micelle concentration (CMC) values associated with low molecular weight surfactant micelles, they are not stable after systemic administration and thus result in the precipitation of solubilized drugs.

Poly(ethylene glycol)(PEG)-lipid conjugates, such as PEG-phosphatidylethanolamie (PEG-PE) have been used for micellar drug delivery [337, 338]. The use of PEG-lipid conjugates for drug delivery are superior to conventional small molecular weight surfactants, since PEG-lipid conjugates have lower critical micelle concentration (CMC: around $10^{-5} \mathrm{M}$ ) than conventional surfactants (CMC: around $\left.10^{-3} \mathrm{M}\right)$, suggesting PEGlipid conjugate micelles are more stable than those formed by conventional surfactants. A typical PEG-lipid conjugate structure includes a hydrophilic PEG corona and a hydrophobic lipid core, which, in turn, is composed of two lipid chains varying from $\mathrm{C} 12$ to $\mathrm{C} 18$ in chain length. Although we could conjugate PEG with different lipids to make different PEG-lipid conjugates, the flexibility in the design of hydrophobic core is still limited.

Alternatively, amphiphilic copolymers have become promising materials for micellar drug delivery. Pluronic is a triblock copolymer composed of a central hydrophobic chain of poly (propylene oxide) (PPO) flanked by two hydrophilic chains of poly (ethylene glycol) (PEG), and has been widely used for drug delivery [339]. It not only increases drug solubility, but also overcomes drug resistance in cancer [340, 341]. The CMC of Pluronic copolymers ranges from $5.3 \times 10^{-3} \mathrm{M}$ to $2.8 \times 10^{-6} \mathrm{M}$ depending on the molecular weight and composition of copolymers [339]. However, the use of Pluronic for drug delivery is somewhat problematic because they are non-biodegradable. Therefore, biodegradable copolymers such as PEG-PLLA-PEG were designed to facilitate the elimination of polymers from the body [342]. In addition, the structure and size of hydrophobic core block could be properly designed to improve the micelle stability and drug loading. A significantly reduced CMC $\left(10^{-6}\right.$ to $\left.10^{-7} \mathrm{M}\right)$ is observed in a diblock copolymer, which is 10 to 100 folds lower than PEG-lipid conjugates [343]. For examples, the CMC of $\mathrm{PEG}_{5100}-\mathrm{PLA}_{4500}$ was around $3 \times 10^{-6} \mathrm{M}$ as determined by

* Adapted with permission. F. Li, M. Danquah, R.I. Mahato, Synthesis and characterization of amphiphilic lipopolymers for micellar drug delivery, Biomacromolecules, 11 (2010) 2610-2620. 
Danquah et al.[321] Similar results were also reported by Yasugi et al.,[339] who showed that the $\mathrm{CMC}$ of $\mathrm{PEG}_{5700}-\mathrm{PLA}_{5400}$ was around $3 \times 10^{-7} \mathrm{M}$. The low CMC of micelles formed by amphiphilic copolymers indicates that they are more stable than PEG-lipid conjugate micelles and can maintain the integrity of micelles upon dilution. In addition, we and others have showed that the drug solubility has been significantly improved by properly designing the hydrophobic core structure to improve the compatibility between the hydrophobic core and drugs [344]. For an example, PEG-b-poly(N-alkyl stearate Laspartamide), a lipid conjugated polymer, has been used to improve the solubility of a hydrophobic drug, amphotericin B, and demonstrated the possibility of using lipopolymers for solubilization of lipid-like drugs [345, 346].

In this study, we synthesized a novel amphiphilic lipopolymer, poly(ethylene glycol)block-poly(2-methyl-2-carboxyl-propylene carbonate-graft-dodecanol) (PEG-PCD). The polymer synthesis procedures were optimized and the properties of polymers were characterized. PEG-PCD lipopolymers could form micelles and effectively incorporate embelin and improve its water solubility. PEG-PCD lipopolymers were compared with similar polymers with different core structures to investigate the effect of core structure on micelle properties, such as stability, drug loading, particle size and morphology, and in vitro drug release. In addition, we also investigated the effect of hydrophobic core size on the properties of PEG-PCD lipopolymer micelles. Finally, the anti-cancer activities of embelin loaded micelles were determined in vitro with prostate cancer cells.

\subsection{Materials and Methods}

\subsubsection{Materials}

Hydroxybenzotriazole (HOBT), 1-ethyl-3-(3-dimethylaminopropyl)carbodiimide (EDC), dodecanol, triethylamine (TEA), stannous 2-ethylhexanoate ( $\left.\mathrm{Sn}(\mathrm{Oct})_{2}\right)$, diethyl zinc ( $\mathrm{Et}_{2} \mathrm{Zn}$ ), 1,8-diazabicyclo[5.4.0]undec-7-ene (DBU), 2,2-bis(hydroxymethyl) propionic acid, methoxy poly (ethylene glycol) $(\mathrm{mPEG}, \mathrm{Mn}=5000, \mathrm{PDI}=1.03)$ and all other reagents were purchased from Sigma Aldrich (St. Louis, MO) and used as received.

6.2.2. Synthesis of poly(ethylene glycol)-block-poly(2-methyl-2-carboxyl-propylene carbonate-graft-dodecanol) (PEG-PCD)

PEG-PCD lipopolymer was synthesized using the following two methods (Figure 6.1):

\subsubsection{Method I}

Poly(ethylene glycol)-block-poly(2-methyl-2-benzoxycarbonyl-propylene carbonate) (PEG-PBC) was synthesized with the following procedures. Monomer 2-methyl-2benzyloxycarbonyl-propylene carbonate (MBC) was synthesized as described by 


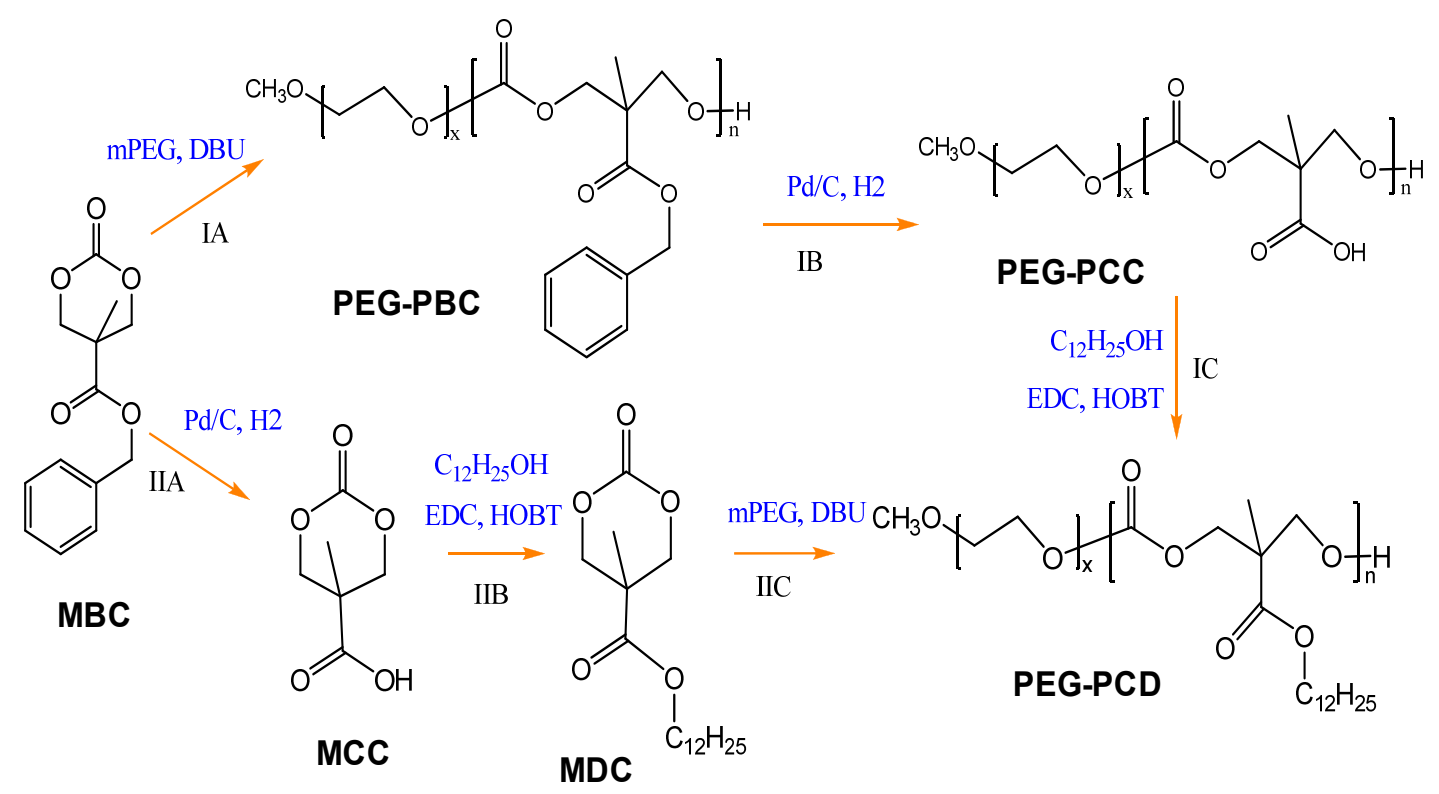

Figure 6.1 Synthesis of PEG-PCD lipopolymer.

Reaction conditions: (IA) DBU, CH2Cl2, RT, 3 h. (IB) Pd/C (10\%), H2 , THF/Methanol(1:1), RT, 18 h.(IC) EDC, HOBT,TEA, DMF, RT, 18 h. (IIA) Pd/C (10\%), H2, EtOAc, RT, 3 h. (IIB) EDC, HOBT,TEA, DMF, RT, 18 h. (IIC) DBU,CH2Cl2, RT, 3 h. 
Danquah et al. [344]. Briefly, a mixture of 0.168 mol 2,2-bis(hydroxymethyl)propionic acid and $0.169 \mathrm{~mol}$ potassiumhydroxide was dissolved in $125 \mathrm{~mL}$ dimethylformamide (DMF) by heating to $100^{\circ} \mathrm{C}$ for $1 \mathrm{~h}$ with stirring. Then, $0.202 \mathrm{~mol}$ benzyl bromide was added drop-wise to the warm solution, and stirred at $100^{\circ} \mathrm{C}$ for $16 \mathrm{~h}$. At the end of reaction, the solvent was removed under reduced pressure. The residue was dissolved in $150 \mathrm{~mL}$ ethyl acetate, washed with water, and dried over $\mathrm{MgSO}_{4}$. The solvent was removed to yield a crude product, which was re-crystallized from toluene to give pure benzyl 2,2-bis(methylol)propionate. Then, $0.05 \mathrm{~mol}$ of benzyl 2,2bis(methylol)propionate was dissolved in $150 \mathrm{~mL} \mathrm{CH}_{2} \mathrm{Cl}_{2}$ containing $25 \mathrm{~mL}$ pyridine, and the solution was chilled to $78^{\circ} \mathrm{C}$. A solution of $\mathrm{CH}_{2} \mathrm{Cl}_{2}$ containing $25 \mathrm{mmol}$ triphosgene was dripped into the solution over $1 \mathrm{~h}$. The reaction mixture was stirred for additional $2 \mathrm{~h}$ at the room temperature before quenched with $75 \mathrm{~mL}$ saturated aqueous $\mathrm{NH}_{4} \mathrm{Cl}$. Subsequently, the organic layer was sequentially washed with aqueous $\mathrm{HCl}(1 \mathrm{M}$, $300 \mathrm{~mL}$ ), saturated aqueous $\mathrm{NaHCO}_{3}(300 \mathrm{~mL})$, dried with $\mathrm{Na}_{2} \mathrm{SO}_{4}$, evaporated to give 2methyl-2-benzyloxycarbonyl-propylene carbonate (MBC) as a crude product, which was further recrystallized from ethyl acetate to give white crystals.

Three methods with different catalysts were investigated in this study to synthesize PEGPBC from copolymerization of mPEG with monomer MBC. (1) $\mathrm{Sn}(\mathrm{Oct})_{2}(10 \mathrm{~mol} \%$ relative to $\mathrm{mPEG}$ ) was added to the mixture of given amount of $\mathrm{mPEG}$ and $\mathrm{MBC}$ dissolved in anhydrous toluene in a dried polymerization flask under the protection of nitrogen atmosphere. To optimize the reaction condition, the reaction mixture was heated to $80{ }^{\circ} \mathrm{C}$ for $3 \mathrm{~h}$ and $16 \mathrm{~h}$ under stirring, respectively. (2) DBU (40 $\left.\mu \mathrm{L}\right)$ was added to the mixture of given amount of mPEG and MBC dissolved in $10 \mathrm{ml}$ anhydrous $\mathrm{CH}_{2} \mathrm{Cl}_{2}$ and reacted at $\mathrm{RT}$ for $3 \mathrm{~h}$ under stirring. (3) $\mathrm{Et}_{2} \mathrm{Zn}$ (10 $\mathrm{mol} \%$ relative to $\left.\mathrm{mPEG}\right)$ was added to the mixture of given amount of MPEG and MBC dissolved in anhydrous toluene in a dried polymerization flask under the protection of nitrogen atmosphere and reacted at 80 ${ }^{\circ} \mathrm{C}$ for $16 \mathrm{~h}$ under stirring. At the end of reaction, the reaction mixture was dissolved in $\mathrm{CHCl}_{3}$ and the product was precipitated with a large amount of ice-cold diethyl ether and dried under vacuum at room temperature.

To remove protective benzyl groups, PEG-PBC was subjected to hydrogenation. Briefly, one gram of PEG-PBC was dissolved in $12 \mathrm{~mL}$ of tetrahydrofuran (THF) and methanol mixture $(1: 1)$ containing $200 \mathrm{mg}$ of palladium on charcoal $(\mathrm{Pd} / \mathrm{C})$. The reaction flask was purged with $\mathrm{N}_{2}$ thrice and charged with $\mathrm{H}_{2}$ using a balloon. The reaction was carried out for $18 \mathrm{~h}$ with stirring. At the end of reaction, $\mathrm{Pd} / \mathrm{C}$ was removed by centrifugation. The solvent was removed under reduced pressure to get poly(ethylene glycol)-block-poly(2methyl-2-carboxyl-propylene carbonate)(PEG-PCC).

Lipid was conjugated to PEG-PCC as described in the following. For lipid conjugation to PEG-PCC, $300 \mathrm{mg}$ PEG-PCC, $260 \mathrm{mg}$ dodecanol, $223 \mathrm{mg}$ hydroxybenzotriazole (HOBT), and $317 \mathrm{mg}$ 1-ethyl-3-(3-dimethylaminopropyl) carbodiimide (EDC) were dissolved in $10 \mathrm{~mL}$ DMF and $340 \mu \mathrm{L}$ of triethylamine (TEA) was then added to the mixture with stirring. After reaction for $18 \mathrm{~h}$, products were precipitated with a large amount of cold isopropyl alcohol twice and diethyl ether once, and then dried under 
vacuum at the room temperature to afford poly(ethylene glycol)-block-poly(2-methyl-2carboxyl-propylene carbonate-graft-dodecanol) (PEG-PCD).

\subsubsection{Method II}

Firstly, 2-methyl-2-carboxyl-propylene carbonate (MCC) was synthesized by hydrogenation of MBC as described previously [344]. Briefly, one gram of monomer MBC was dissolved in $10 \mathrm{ml}$ ethyl acetate containing $100 \mathrm{mg}$ of $\mathrm{Pd} / \mathrm{C}$. The reaction flask was purged with $\mathrm{N}_{2}$ three times and charged with $\mathrm{H}_{2}$ to 45 psi. The reaction was carried out for $3 \mathrm{~h}$. Then, $\mathrm{Pd} / \mathrm{C}$ was removed by centrifugation. The solvent was removed under reduced pressure to give MTC-OH as white crystal.

Then, 2-methyl-2-dodecanoxycarbonyl-propylene carbonate (MDC) was synthesized. To synthesize MDC, $480 \mathrm{mg}$ MTC-OH, $465 \mathrm{mg}$ dodecanol, $506 \mathrm{mg}$ HOBT, and $720 \mathrm{mg}$ EDC were dissolved in $20 \mathrm{ml} \mathrm{DMF}$ and $490 \mu \mathrm{L}$ of TEA was then added to the mixture with stirring for $18 \mathrm{~h}$. The reaction mixture was diluted with ethyl acetate $(20 \mathrm{~mL})$ and washed with water and dried over $\mathrm{MgSO}_{4}$. The solvent was removed under reduced pressure to yield a crude product, which was further purified by column chromatography to get MDC as white powder.

Finally, PEG-PCD was prepared from copolymerization of mPEG with MDC. DBU (40 $\mu \mathrm{L}$ ) was added to the mixture of a given amount of $\mathrm{mPEG}$ and MDC was dissolved in anhydrous $\mathrm{CH}_{2} \mathrm{Cl}_{2}$ and reacted under stirring for $3 \mathrm{~h}$. The reaction mixture was dissolved in $\mathrm{CHCl}_{3}$ and the product was precipitated with a large amount of cold isopropyl alcohol, diethyl ether, and dried under vacuum at room temperature to get PEG-PCD lipopolymer.

\subsubsection{Polymer characterization}

\subsubsection{Nuclear magnetic resonance (NMR)}

${ }^{1} \mathrm{H}$ NMR spectra were recorded on Varian (500 MHz) using deuterated chloroform $\left(\mathrm{CDCl}_{3}\right)$ as a solvent unless otherwise noted. The chemical shifts were calibrated using tetramethylsilane as an internal reference and given in parts per million.

\subsubsection{Infrared (IR) spectra}

The composition of synthesized polymers was also confirmed with Infra red (IR) spectra using a Perkin-Elmer IR spectrometer.

\subsubsection{Gel permeation chromatography (GPC)}

The weight $\left(\mathrm{M}_{\mathrm{w}}\right)$ and number $\left(\mathrm{M}_{\mathrm{n}}\right)$ average molecular weight and polydispersity index (PDI) of synthesized polymers were determined by a Waters GPC system equipped with a GPC column (AM Gel 10\%/5) and a differential refractive index detector. THF was used 
as an eluent at a flow rate of $1 \mathrm{ml} / \mathrm{min}$. A series of narrow polystyrene standards $(700$ $40,000 \mathrm{~g} / \mathrm{mol}$ ) were used for calibration.

\subsubsection{Differential scanning calorimetry}

The thermal properties, including glass transition temperature $\left(\mathrm{T}_{\mathrm{g}}\right)$, melting temperature $\left(T_{m}\right)$, crystalline temperature $\left(T_{c}\right)$ of the synthesized polymers were determined by differential scanning calorimetry (DSC) (TA Instrument DSC Q2000 module). Samples were placed in aluminum pans under nitrogen heated from $25^{\circ} \mathrm{C}$ to $100{ }^{\circ} \mathrm{C}$, cooled to -70 ${ }^{\circ} \mathrm{C}$ to remove thermal history and heated from $-70{ }^{\circ} \mathrm{C}$ to $100{ }^{\circ} \mathrm{C}$ at a rate of $5{ }^{\circ} \mathrm{C} / \mathrm{min}$.

\subsubsection{Critical micelle concentration}

Critical micelle concentration (CMC) was determined with fluorescent spectroscopy using pyrene as a hydrophobic fluorescent probe, as previously described [347]. Briefly, forty microliters of pyrene stock solution $\left(2.4 \times 10^{-3} \mathrm{M}\right)$ in acetone was added to $40 \mathrm{~mL}$ water to prepare a saturated pyrene aqueous solution. Polymer samples were dispersed in water with concentration ranges from 0.5 to $4.8 \times 10^{-7} \mathrm{mg} / \mathrm{mL}$ and mixed thoroughly with above pyrene solution at a volume ratio of 1:1. The fluorescent intensity was recorded with a Molecular Devices SpectraMax M2/M2e spectrofluorometer (Sunnyvale, CA) with a $E_{X}=338 \mathrm{~nm}\left(\mathrm{I}_{3}\right)$ and $333\left(\mathrm{I}_{1}\right), \mathrm{E}_{\mathrm{m}}=390 \mathrm{~nm}$. The intensity ratio $\left(\mathrm{I}_{338} / \mathrm{I}_{333}\right)$ was plotted against the logarithm of polymer concentration. The CMC value was obtained as the point of intersection of two tangents drawn to the curve at high and low concentrations, respectively.

\subsubsection{Preparation of polymeric micelles}

Polymeric micelles were prepared with a film dispersion method as previously reported with some modifications [348]. Briefly, $15 \mathrm{mg}$ of polymer and given amount of embelin were dissolved in $0.5 \mathrm{~mL} \mathrm{CH}_{2} \mathrm{Cl}_{2}$, and then the solvent was removed under reduced

pressure. The resulting film was hydrated in $3 \mathrm{~mL}$ PBS ( $\mathrm{pH} 7.4)$ and sonicated for $1 \mathrm{~min}$. Then, the residue free drug was removed by centrifugation at 12,000 rpm for $5 \mathrm{~min}$. The supernatant was filtered using a $0.22 \mu \mathrm{M}$ filter.

\subsubsection{Drug loading and encapsulation efficiency}

To determine drug loading, $10 \mu \mathrm{L}$ of embelin loaded micelle solution was diluted with methanol. Then drug concentration was determined using a UV spectrometer at $310 \mathrm{~nm}$ (Thermo Spectronic). Embelin concentration was calculated based the UV absorbance using a standard curve. Drug loading and encapsulation efficiency were then determined using the following equations, respectively. Drug loading $(\%)=(($ amount of loaded drug $)$ $/($ amount of polymer $)) \times 100 \%$. Drug encapsulation efficiency $(\%)=(($ amount of loaded 
drug $) /($ amount of drug added $)) \times 100 \%$. Theoretical loading $(\%)=(($ amount of drug added) $/($ amount of polymer $)) \times 100 \%$.

\subsubsection{Morphology and particle size}

The particle size of polymeric micelles was determined by dynamic light scattering (Malvern Nano ZS). The intensity of scattered light was detected at $90^{\circ}$. The particle size measurement was repeated three times and the data were reported as the mean $\pm \mathrm{SD}$. The morphology of polymeric micelles were observed using a transmission electron microscope (TEM, JEM-100S) using an acceleration voltage of $60 \mathrm{kV}$. Micelles was loaded on a copper grid and stained with $1 \%$ uranyl acetate. The grid was visualized under the electron microscope with magnifications of 75,000.

\subsubsection{In vitro drug release}

The dialysis technique was employed to determine the release of embelin from polymeric micelles in PBS (pH 7.4) with 0.1\% Tween 80. The micelles used for in vitro release study have a polymer concentration of $5 \mathrm{mg} / \mathrm{mL}$. Eight hundred microliters of micelles with 5\% drug loading were used to compare the release profiles of PEG-PCD with different PCD length. In addition, $2 \mathrm{~mL}$ micelles with $2 \%$ drug loading were also used to compare the release profiles of $\mathrm{PEG}_{114}-\mathrm{PCD}_{29}$ and $\mathrm{PEG}_{114}-\mathrm{PBC}_{30}$. Embelin-loaded micelles were placed into a dialysis tube with a molecular weight cut-off of $3500 \mathrm{Da}$ and dialyzed against $50 \mathrm{~mL}$ release medium in a thermo-controlled shaker with a stirring speed of $180 \mathrm{rpm}$ at $37^{\circ} \mathrm{C}$. At specified time, $1 \mathrm{~mL}$ release medium was withdrawn and embelin concentration was determined with a UV spectrophotometer based on UV absorbance at $310 \mathrm{~nm}$. All experiments were performed in triplicate and the data reported as the mean of the three individual experiments. The release profiles were assessed as described previously [349] using similarity factor (f2). The percentage of cumulative drug release was calculated with the following equation: cumulative drug release $(\%)=$ ((cumulative amount of released drug) / (total amount of drug added $)) \times 100 \%$.

\subsubsection{In vitro cytotoxicity of embelin loaded micelles}

C4-2 prostate cancer cell line was used to determine the cell growth inhibition ability of embelin-loaded micelles. Cells were cultured in RPMI 1640 media supplemented with $10 \%$ fetal bovine serum (FBS) and $1 \%$ antibiotic-antimycotic at $37{ }^{\circ} \mathrm{C}$ in humidified environment of $5 \% \mathrm{CO}_{2}$. Cells were seeded in 96-well plates at a density of 5,000 cells per well $18 \mathrm{~h}$ before treatment. Then, cells were treated with drug-loaded micelles or equivalent amount of blank micelles for additional $72 \mathrm{~h}$. At the end of treatment, cell culture medium was replaced by $100 \mu \mathrm{L}$ medium with $0.5 \mathrm{mg} / \mathrm{mL} 3$-(4,5-dimethylthiazol-2-yl)-2, 5-diphenyl tetrazolium bromide (MTT) and incubated for $1 \mathrm{~h}$ at $37{ }^{\circ} \mathrm{C}$. Then the medium was carefully removed and $200 \mu \mathrm{L}$ of DMSO was added into each well to dissolve the formazan crystals. The absorbance was measured in a microplate reader at 
a wavelength of $560 \mathrm{~nm}$. Cell viability was expressed as the percentage of control group. Cell viability $(\%)=\left(\mathrm{A}_{\text {test }} / \mathrm{A}_{\text {control }}\right) \times 100 \%$.

\subsection{Results}

\subsubsection{Synthesis and characterization of polymers}

As summarized in Figure 6.1, the target lipopolymer PEG-PCD was synthesized by two approaches. In the first approach, PEG was copolymerized with MBC to afford poly(ethylene glycol)-block-poly(2-methyl-2-benzoxycarbonyl-propylene carbonate) (PEG-PBC). Then, the pendant benzyl group was removed by hydrogenation to expose carboxyl groups to get poly(ethylene glycol)-block-poly(2-methyl-2-carboxyl-propylene carbonate)(PEG-PCC). Finally, poly(ethylene glycol)-block-poly(2-methyl-2-carboxylpropylene carbonate-graft-dodecanol) (PEG-PCD) lipopolymer was synthesized by conjugating dodecanol to the carboxyl groups. In the second approach, PEG was copolymerized with a lipid modified monomer 2-methyl-2-dodecanoxycarbonylpropylene carbonate (MDC). MCC and MDC were characterized with ${ }^{1} \mathrm{H}$ NMR and ESIMS: MCC, ${ }^{1} \mathrm{H}$ NMR (500 MHz, DMSO): $\delta 13.35$ (s, $\left.1 \mathrm{H}\right), 4.57$ (d, $\left.2 \mathrm{H}\right), 4.32$ (d, $2 \mathrm{H}$ ), 1.18(s, 3 H). MS (ESI) m/z 159 [M-H] ${ }^{-}$MCC, ${ }^{1} \mathrm{H}_{\mathrm{NMR}}\left(500 \mathrm{MHz}, \mathrm{CDCl}_{3}\right): \delta 4.50$ (d, 2 H), $4.2(\mathrm{~d}, 4 \mathrm{H}), 1.65(\mathrm{~m}, 2 \mathrm{H}), 1.4-1.2(\mathrm{~m}, 21 \mathrm{H}), 0.85$ (t, $3 \mathrm{H}) . \mathrm{MS}(\mathrm{ESI}) \mathrm{m} / \mathrm{z} 351[\mathrm{M}+$ $\mathrm{Na}]^{+}$. Although we could synthesize the lipopolymers with both approaches, the second approach involves column purification of MDC which is a time consuming process. Therefore, the first approach was selected to generate lipopolymer PEG-PCD.

To achieve the optimal polymerization efficiency, three different catalysts were tested for copolymerization of PEG and MBC as summarized in Table 6.1. Among three catalysts tested, DBU was the best, as the conversion rate was $80-88 \%$ at the molar ratio of monomer and PEG ranging from 10 to 34 . Both $\mathrm{Sn}(\mathrm{oct})_{2}$ and $\mathrm{Et}_{2} \mathrm{Zn}$ were used at $80{ }^{\circ} \mathrm{C}$ and the degree of polymerization (DP) was increased with prolonged reaction time from $3 \mathrm{~h}$ to $16 \mathrm{~h}$ for $\mathrm{Sn}(\mathrm{oct})_{2}$. However, both of these two catalysts gave less DP under the reaction conditions described in Table 6.1. Therefore, DBU was selected in the current study.

The structures of PEG-PBC, PEG-PCC, and PEG-PCD were confirmed by ${ }^{1} \mathrm{H}$ NMR (Figure 6.2). From Figure 6.2A, the following peaks were observed for PEG-PBC polymer at $\delta 1.2\left(\mathrm{CH}_{3}\right.$ in $\mathrm{BC}$ unit), $\delta 3.6\left(\mathrm{CH}_{2}\right.$ in $\left.\mathrm{PEG}\right), \delta 4.3\left(\mathrm{CH}_{2}\right.$ in $\mathrm{BC}$ main chain), $\delta$ $5.2\left(\mathrm{CH}_{2}\right.$ in $\mathrm{BC}$ side group), and $\delta 7.3$ (phenyl ring). All signals are assigned as methoxy poly(ethylene glycol) (mPEG) and polymerized BC units. In addition, the success of polymerization was confirmed by the disappearance of signal at $\delta 4.2$ and $\delta 4.6$ in the MBC monomer and appearance of a new peak at $\delta 4.3$. The degree of polymerization and molecular weight of the polymers was estimated based on the peak areas of $\mathrm{PEG} \mathrm{CH}_{2}$ groups at $\delta 3.6$ and those of $\mathrm{CH}_{2}$ in $\mathrm{BC}$ main chain at $\delta 4.3$. 
Table 6.1 Effect of different catalyst on the polymerization of PEG-PBC copolymer.

\begin{tabular}{cccccc}
\hline Catalyst $^{a}$ & $\begin{array}{c}{[\mathrm{MBC}] /} \\
{[\mathrm{PEG}-\mathrm{OH}]^{e}}\end{array}$ & $\mathrm{M}_{\mathrm{n}, \text { Target }}^{f}$ & $\mathrm{DP}_{\mathrm{MBC}^{g}}$ & $\mathrm{M}_{\mathrm{n}}{ }^{h}$ & $\begin{array}{c}\text { Conversion } \\
(\%)^{i}\end{array}$ \\
\hline $\mathrm{DBU}^{b}$ & 10 & 7500 & 8 & 7000 & 80 \\
$\mathrm{DBU}^{b}$ & 20 & 10000 & 17 & 9250 & 85 \\
$\mathrm{DBU}^{b}$ & 34 & 13500 & 30 & 12500 & 88 \\
$\mathrm{Sn}(\mathrm{oct})_{2}{ }^{c}$ & 34 & 13500 & 17 & 9250 & 50 \\
$\mathrm{Sn}(\mathrm{oct})_{2}{ }^{d}$ & 34 & 13500 & 22 & 10500 & 65 \\
$\mathrm{Et}_{2} \mathrm{Zn}^{d}$ & 20 & 10000 & 6 & 6500 & 30 \\
\hline
\end{tabular}

a. DBU: 1,8-diazabicyclo[5.4.0]undec-7-ene; $\mathrm{Sn}(\mathrm{oct})_{2}$ : stannous 2-ethylhexanoate; $\mathrm{Et}_{2} \mathrm{Zn}$ : diethy zinc.

b. Reaction condition: RT, $3 \mathrm{~h}$.

c. Reaction condition: $80^{\circ} \mathrm{C}, 3 \mathrm{~h}$.

d. Reaction condition: $80^{\circ} \mathrm{C}, 16 \mathrm{~h}$.

e. Molar ratio of monomer (MBC) to PEG in the feed.

f. $\mathrm{M}_{\mathrm{n} \text {, target }}=[\mathrm{MBC}] /[\mathrm{PEG}-\mathrm{OH}] \times \mathrm{M}_{\text {monomer unit }}(250)+\mathrm{M}_{\text {PEG-OH }}(5000)$.

g. DP: Degree of Polymerization, determined by ${ }^{1} \mathrm{H}$ NMR.

h. $\mathrm{M}_{\mathrm{n}}=\mathrm{DP} \times \mathrm{M}_{\text {monomer unit }}(250)+\mathrm{M}_{\text {PEG-OH }}(5000)$.

i. Determine by ${ }^{1} \mathrm{H}$ NMR, Conversion $(\%)=\mathrm{DP} /([\mathrm{MBC}] /[\mathrm{PEG}-\mathrm{OH}]) \times 100 \%$. 

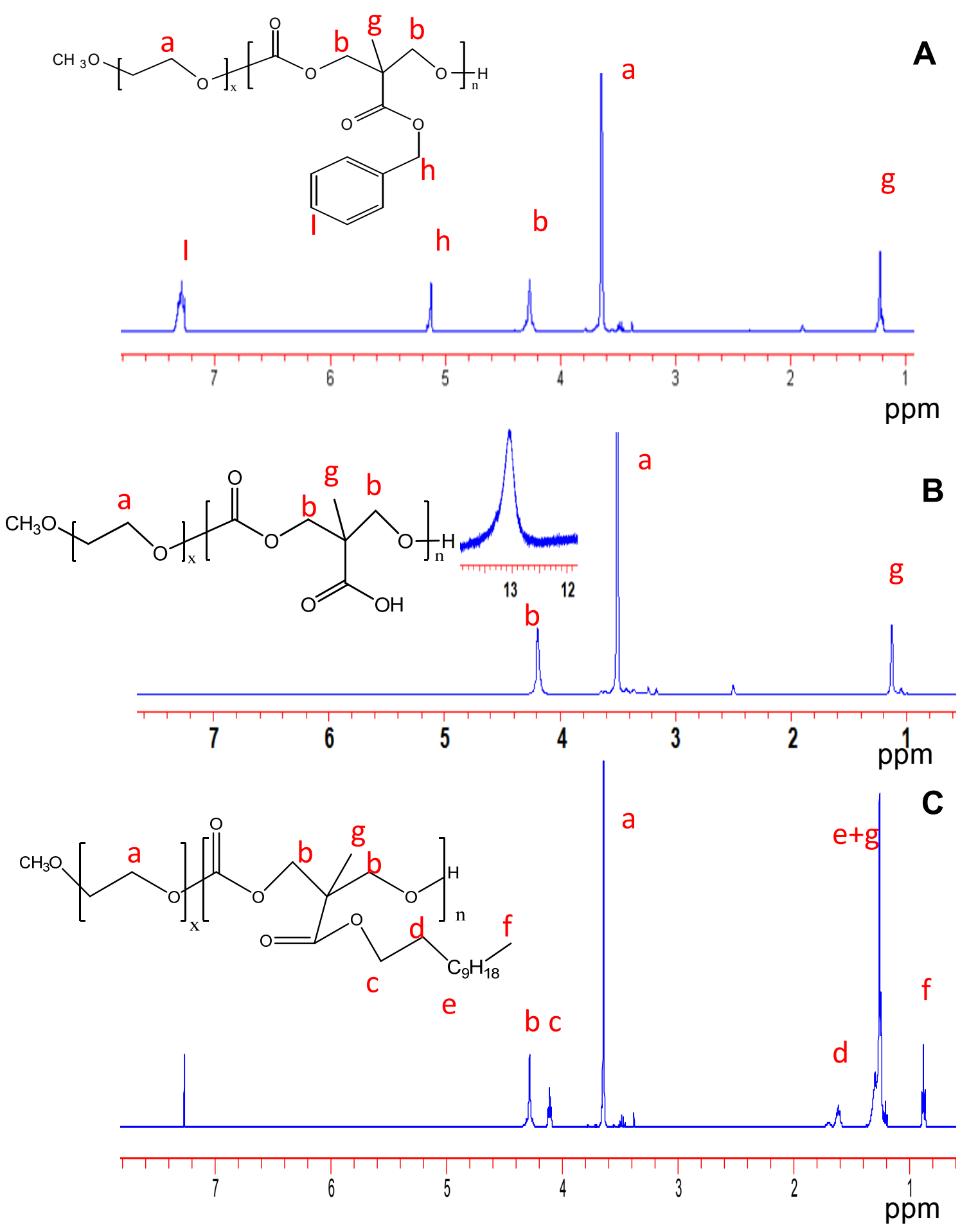

Figure 6.2 ${ }^{1}$ H NMR of polymers.

(A) $\mathrm{PEG}_{114}-\mathrm{PBC}_{30}$ in $\mathrm{CDCl}_{3}$, (B) $\mathrm{PEG}_{114}-\mathrm{PCC}_{30}$ in $\mathrm{DMSO}$, (C) $\mathrm{PEG}_{114}-\mathrm{PCD}_{29}$ in $\mathrm{CDCl}_{3}$. 
The benzyl groups were removed via $\mathrm{Pd} / \mathrm{C}$ catalyzed hydrogenation. The peaks at $\delta 5.2$ $\left(\mathrm{CH}_{2}\right.$ in $\mathrm{BC}$ side group) and $\delta 7.3$ (phenyl ring) disappeared after hydrogenation (Figure $6.2 \mathrm{~B}$ ), which indicates the complete removal of pendant benzyl group. In addition, a peak at $\delta 13$ was observed, which belongs to the exposed carboxyl group after removing protective benzyl group. Dodecanol was conjugated onto the polymer backbone using EDC/HOBT chemistry. The success of dodecanol conjugation to PEG-PCC carboxyl group was confirmed by the appearance of new peaks at $\delta 4.1, \delta 1.6, \delta 1.2-1.4$, and $\delta 0.9$, which could be assigned to different groups in the lipid as shown in Figure 6.2C.

The FTIR of PEG-PBC, PEG-PCC, and PEG-PCD also confirmed the success of the reaction (Figure 6.3). In FTIR spectra of PEG-PBC, there were peaks at 736 and 696 $\mathrm{cm}^{-1}$, which were due to the $\mathrm{CH}$ vibrations of benzyl groups. These two peaks were absent in the FTIR spectra of PEG-PCC and PEG-PCD, indicating the removal of the pendant benzyl groups during hydrogenation. In addition, we also observed a broad $\mathrm{OH}$ stretch band, which was centered at $3450 \mathrm{~cm}^{-1}$ and partially overlapped with C-H stretch. This further confirmed the presence of pendant $\mathrm{COOH}$ groups. Besides, we also observed absorbance bands due to the $\mathrm{C}=\mathrm{O}$ stretching vibration at $1749 \mathrm{~cm}^{-1}$ (PEGPBC), $1748 \mathrm{~cm}^{-1}$ (PEG-PCC), and $1755 \mathrm{~cm}^{-1}$ (PEG-PCD), respectively. The C-H stretch band was observed at $2886 \mathrm{~cm}^{-1}$ for PEG-PBC, $2874 \mathrm{~cm}^{-1}$ for PEG-PCC. In addition, 3 bands at 2923, 2889, and $2855 \mathrm{~cm}^{-1}$ were observed for PEG-PCD, indicating the presence of pendant long alkanes.

The characteristics of PEG-PBC, PEG-PCC, and PEG-PCD polymers are summarized in Table 6.2. The molecular weight of PEG-PBC and PEG-PCC were calculated based on the peak areas of $\mathrm{PEG} \mathrm{CH}_{2}$ groups at $\delta 3.6$ and those of $\mathrm{CH}_{2}$ in $\mathrm{BC}$ main chain at $\delta 4.3$ in the ${ }^{1} \mathrm{H}$ NMR spectrum. In addition, the percentage of conjugation and molecular weight of the lipopolymer was calculated by the peak areas at $\delta 4.3$ (belongs to polymer backbone) and at $\delta 4.1$ (belongs to the conjugated pendant lipid) in the ${ }^{1} \mathrm{H}$ NMR spectrum. The $\mathrm{M}_{\mathrm{n}}$ determine by ${ }^{1} \mathrm{H}$ NMR were $12,500 \mathrm{~g} / \mathrm{mol}$ for $\mathrm{PEG}_{114}-\mathrm{PBC}_{30}, 9,800$ $\mathrm{g} / \mathrm{mol}$ for $\mathrm{PEG}_{114}-\mathrm{PCC}_{30}, 13,561 \mathrm{~g} / \mathrm{mol}$ for $\mathrm{PEG}_{114}-\mathrm{PCD}_{29}, 10,248 \mathrm{~g} / \mathrm{mol}$ for $\mathrm{PEG}_{114-}$ $\mathrm{PCD}_{16}$, and $6,968 \mathrm{~g} / \mathrm{mol}$ for $\mathrm{PEG}_{114}-\mathrm{PCD}_{6}$, respectively. GPC was also used to determine the apparent weight $\left(\mathrm{M}_{\mathrm{w}}\right)$ and number $\left(\mathrm{M}_{\mathrm{n}}\right)$ average molecular weight of polymers and their polydispersity index. A good correlation was observed between the molecular weight estimated from NMR and Mw or Mn determined by GPC. All of the polymers showed narrow distribution as revealed by the small polydispersity index (1.06 to 1.23). A representative plot comparing the GPC chromatograms for $\mathrm{PEG}_{114}-\mathrm{PCC}_{30}, \mathrm{PEG}_{114^{-}}$ $\mathrm{PBC}_{30}$, and $\mathrm{PEG}_{114}-\mathrm{PCD}_{29}$ was shown in Figure 6.4.

DSC was used to determine the thermal properties of the synthesized block copolymers. The thermograms of diblock copolymers with different core structures are shown in Figure 6.5. $\mathrm{PEG}_{114}-\mathrm{PBC}_{30}$ has a $\mathrm{T}_{\mathrm{g}}=-35.7^{\circ} \mathrm{C}, \mathrm{T}_{\mathrm{c}}=6.3^{\circ} \mathrm{C}$, and $\mathrm{T}_{\mathrm{m}}=49.5^{\circ} \mathrm{C}$, respectively. $\mathrm{PEG}_{114}-\mathrm{PCC}_{30}$, which is a transparent viscous liquid, showed a $\mathrm{T}_{\mathrm{g}}$ of $-30.7^{\circ} \mathrm{C}$, but no obvious $\mathrm{T}_{\mathrm{c}}$ or $\mathrm{T}_{\mathrm{m}} \cdot \mathrm{PEG}_{114}-\mathrm{PCD}_{29}$ showed a $\mathrm{T}_{\mathrm{m}}$ of $-35.7^{\circ} \mathrm{C}$, but no obvious $\mathrm{T}_{\mathrm{g}}$ or $\mathrm{T}_{\mathrm{c}}$. The different thermal properties observed with DSC suggesting different properties of the copolymers. Only $\mathrm{T}_{\mathrm{g}}$ was observed in $\mathrm{PEG}_{114}-\mathrm{PCC}_{30}$, indicating it is an amorphous polymer. $\mathrm{PEG}_{114}-\mathrm{PCD}_{29}$ has a high degree of crystallinity, since there was no $\mathrm{T}_{\mathrm{g}}$. 


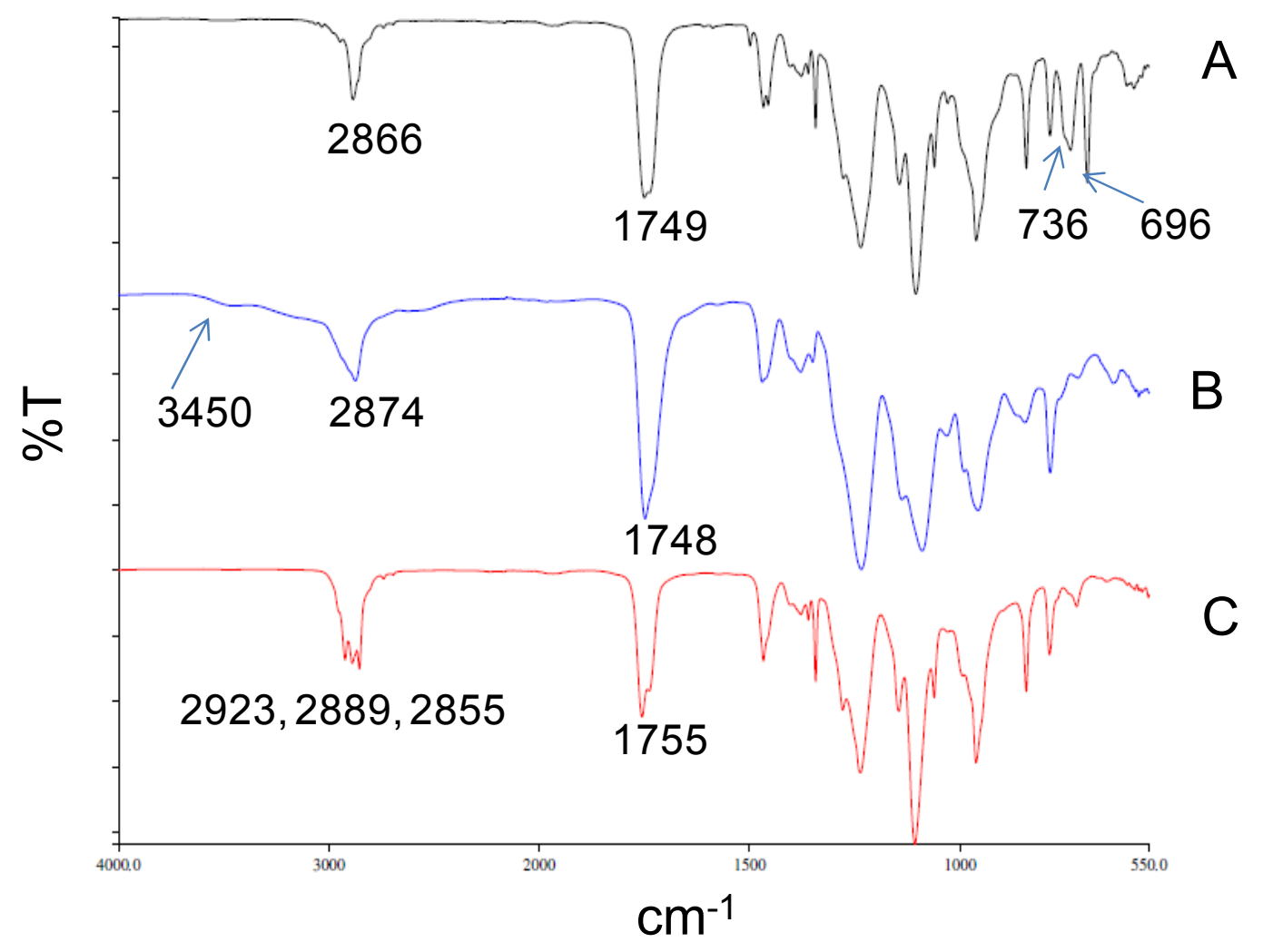

Figure 6.3 FTIR spectra of polymers.

(A) $\mathrm{PEG}_{114}-\mathrm{PBC}_{30}$, (B) $\mathrm{PEG}_{114}-\mathrm{PCC}_{30}$, and (C) $\mathrm{PEG}_{114}-\mathrm{PCD}_{29}$. 
Table 6.2 Characteristics of PEG copolymers with different core structure.

\begin{tabular}{|c|c|c|c|c|c|c|c|}
\hline Polymer $^{a}$ & $\begin{array}{c}\mathrm{M}_{\mathrm{n}} \\
(\mathrm{NMR})\end{array}$ & $\begin{array}{c}\mathrm{M}_{\mathrm{w}} \\
(\mathrm{GPC})^{b}\end{array}$ & $\begin{array}{c}\mathrm{M}_{\mathrm{n}} \\
(\mathrm{GPC})^{b}\end{array}$ & $\mathrm{PDI}^{b}$ & $\begin{array}{l}\text { CMC } \\
(\mathrm{g} / \mathrm{L})\end{array}$ & $\begin{array}{l}\mathrm{CMC} \\
(\mathrm{M})^{c}\end{array}$ & Crystallinity \\
\hline $\mathrm{PEG}_{114}-\mathrm{PBC}_{30}$ & 12500 & 11140 & 9703 & 1.15 & $4.0 \times 10^{-4}$ & $3.2 \times 10^{-8}$ & semicrystalline \\
\hline $\mathrm{PEG}_{114}-\mathrm{PCC}_{30}$ & 9800 & 7447 & 6563 & 1.13 & $2.2 \times 10^{-2}$ & $2.3 \times 10^{-6}$ & amorphous \\
\hline $\mathrm{PEG}_{114}-\mathrm{PCD}_{29}$ & 13561 & 13342 & 11636 & 1.15 & $3.6 \times 10^{-4}$ & $2.6 \times 10^{-8}$ & crystalline \\
\hline $\mathrm{PEG}_{114}-\mathrm{PCD}_{16}$ & 10248 & 11794 & 9590 & 1.23 & $9.0 \times 10^{-4}$ & $8.7 \times 10^{-8}$ & crystalline \\
\hline $\mathrm{PEG}_{114}-\mathrm{PCD}_{6}$ & 6968 & 6580 & 6203 & 1.06 & $1.4 \times 10^{-3}$ & $2.0 \times 10^{-7}$ & crystalline \\
\hline
\end{tabular}

a. PEG-PBC: poly(ethylene glycol)-block-poly(2-methyl-2-benzoxycarbonyl-propylene carbonate); PEG-PCD: poly(ethylene glycol)block-poly(2-methyl-2-carboxyl-propylene carbonate-graft-dodecanol); PEG-PCC: poly(ethylene glycol)-block-poly(2-methyl-2carboxyl-propylene carbonate).

b. $M_{w}$ and $M_{n}$ was apparent molecular weight determined by GPC; PDI $=\left(M_{w} / M_{n}\right) \times 100 \%$.

c. $\mathrm{CMC}(\mathrm{g} / \mathrm{L})$ normalized with $\mathrm{Mn}$ from ${ }^{1} \mathrm{H} \mathrm{NMR}$. 


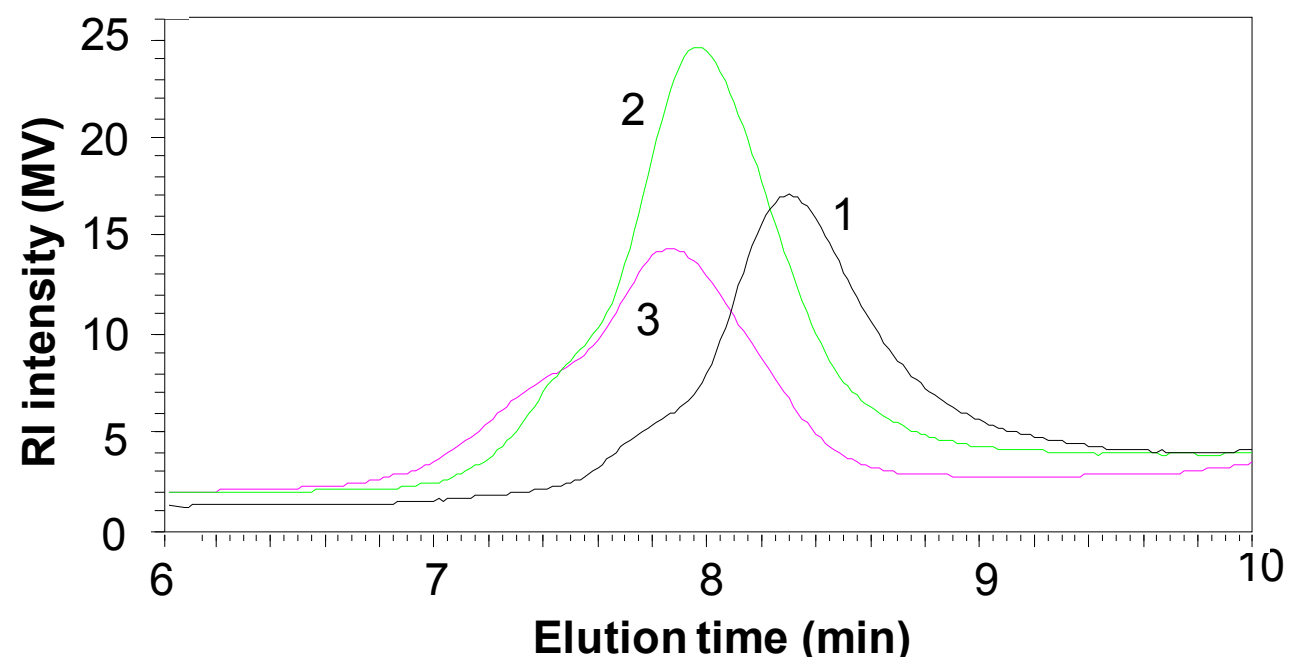

Figure 6.4 Gel permeation chromatography (GPC) of polymers.

(1) $\mathrm{PEG}_{114}-\mathrm{PCC}_{30}[\mathrm{Mn}=6563 \mathrm{~g} / \mathrm{mol}, \mathrm{Mw} / \mathrm{Mn}=1.13]$; (2) $\mathrm{PEG}_{114}-\mathrm{PBC}_{30}[\mathrm{Mn}=9703$ $\mathrm{g} / \mathrm{mol}, \mathrm{Mw} / \mathrm{Mn}=1.15]$; (3) $\mathrm{PEG}_{114}-\mathrm{PCD}_{29}[\mathrm{Mn}=11636 \mathrm{~g} / \mathrm{mol}, \mathrm{Mw} / \mathrm{Mn}=1.15]$. 


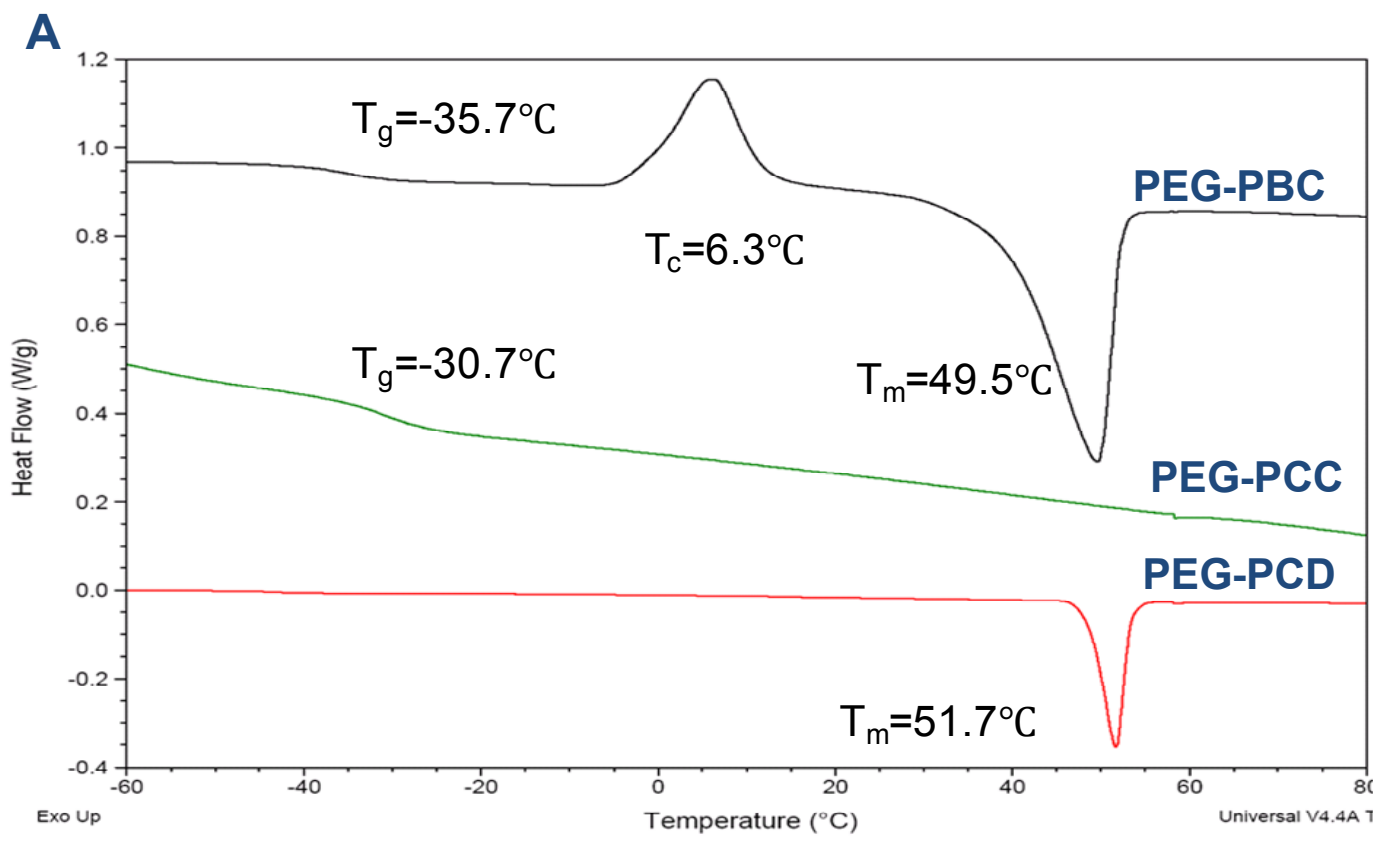

B

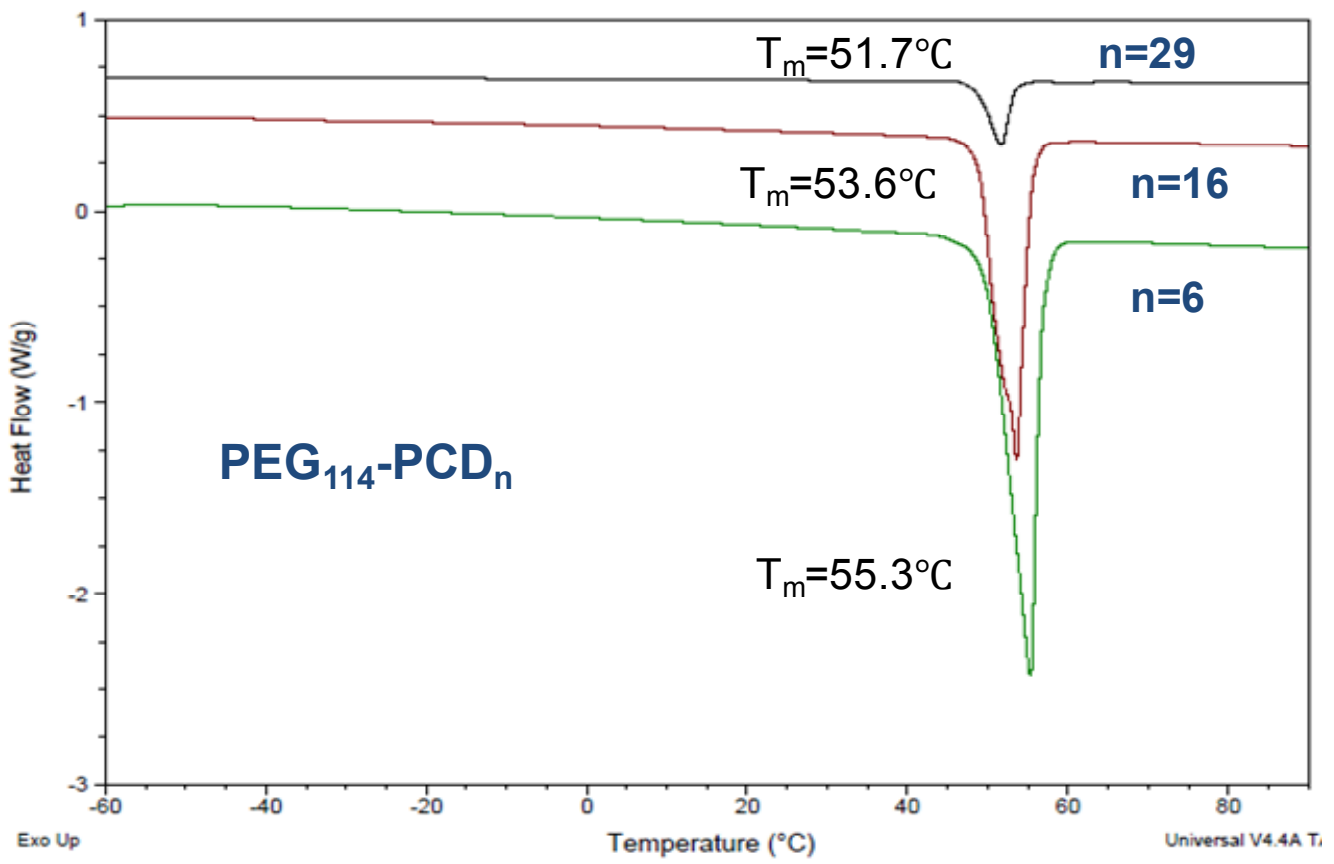

Figure 6.5 Thermal analysis of polymers by DSC.

(A) Polymers of different core structures: $\mathrm{PEG}_{114}-\mathrm{PBC}_{30}, \mathrm{PEG}_{114}-\mathrm{PCC}_{30}$, and $\mathrm{PEG}_{114}$ $\mathrm{PCD}_{29}$. (B) PEG-PCD polymers with different PCD core length: $\mathrm{n}=6,16$, and 29. 
The observed $T_{g}, T_{c}$, and $T_{m}$ in PEG-PBC indicate the existence of both amorphous domain and crystalline domains in $\mathrm{PEG}_{114}-\mathrm{PBC}_{30}$, which is a semi-crystalline polymer. We also calculated the degree of crystallinity based on the DSC peak areas, which was $57.1 \%$. Different degrees of crystallinity of $\mathrm{PEG}_{114}-\mathrm{PCC}_{30}, \mathrm{PEG}_{114}-\mathrm{PBC}_{30}$, and $\mathrm{PEG}_{114}$ $\mathrm{PCD}_{29}$, might be due to the different intermolecular forces among these three polymers with different hydrophobic core structures. We also determined the effect of hydrophobic core size on the $T_{m}$ of PEG-PCD. As shown in Figure 6.5B, the $T_{m}$ for $P_{E G} G_{114}-P D_{29}$, $\mathrm{PEG}_{114}-\mathrm{PCD}_{16}$, and $\mathrm{PEG}_{114}-\mathrm{PCD}_{6}$ were $51.7^{\circ} \mathrm{C}, 53.6^{\circ} \mathrm{C}, 55.3{ }^{\circ} \mathrm{C}$, respectively.

The polymers were further characterized by fluorescence spectroscopy to determine the CMC (Figure 6.6). $\mathrm{PEG}_{114}-\mathrm{PBC}_{30}$ and $\mathrm{PEG}_{114}-\mathrm{PCD}_{29}$ had low $\mathrm{CMC}$ values of $4.0 \times 10^{-4}$ $\mathrm{g} / \mathrm{L}$ and $3.6 \times 10^{-4} \mathrm{~g} / \mathrm{L}$, respectively. In contrast, the $\mathrm{CMC}$ of $\mathrm{PEG}_{114}-\mathrm{PCC}_{30}$ was $2.2 \times 10^{-}$ ${ }^{2} \mathrm{~g} / \mathrm{L}$, which was around 50 folds higher than those of $\mathrm{PEG}_{114}-\mathrm{PBC}_{30}$ or $\mathrm{PEG}_{114}-\mathrm{PCD}_{29}$. The low $\mathrm{CMC}$ indicates the good stability of micelles prepared from $\mathrm{PEG}_{114}-\mathrm{PBC}_{30}$ and $\mathrm{PEG}_{114}-\mathrm{PCD}_{29}$. The effect of hydrophobic core size on PEG-PCD micelle stability was also determined. The CMC of PEG-PCD micelles decreased significantly from $1.4 \times 10^{-3}$ $\mathrm{g} / \mathrm{L}$ to $3.6 \times 10^{-4} \mathrm{~g} / \mathrm{L}$, when the DP(n) increased from 6 to 29 (Figure $6.6 \mathrm{~B}$ ).

\subsubsection{Preparation and characterization of micelles}

\subsubsection{Drug loading efficiency}

The amount of embelin loaded into polymeric micelles and loading efficiency were calculated using the equations described in the experimental methods. As demonstrated in Figure 6.7, PEG-PBC showed $2 \%$ drug loading at 5\% theoretical loading, which corresponds to $40 \%$ loading efficiency, in contrast, $5 \%$ drug loading was achieved by PEG-PCD with different hydrophobic core lengths, which corresponds to $100 \%$ loading efficiency. Increase in theoretical loading from 5\% to $10 \%$ showed little effect on the drug loading of PEG-PBC micelles and the loading efficiency decreased correspondingly. However, the drug loading increased from 5\% to $6 \%$ and $7 \%$ for PEG-PCD micelles, when theoretical loading increased from $5 \%$ to $10 \%$ and $15 \%$, with decrease in loading efficiency from $100 \%$ to $60 \%$ and $50 \%$, respectively. There was no significant difference among three PEG-PCD lipopolymers with different hydrophobic core lengths.

\subsubsection{Core-shell structure formation}

${ }^{1} \mathrm{H}$ NMR spectroscopy was used to demonstrate the formation of core-shell of PEG-PCD lipopolymer micelles (Figure 6.8). In $\mathrm{CDCl}_{3}$, peaks from both the hydrophilic PEG block (3.6 ppm) and the hydrophobic core block (4.3, 4.1, 1.6, 1.2-1.4, and $0.9 \mathrm{ppm})$ was observed. In $\mathrm{D}_{2} \mathrm{O}$, all the peaks corresponding to the hydrophobic core block were significantly diminished in contrast to the strong peaks for hydrophilic PEG block (Figure 6.8A). This is consistent with a core-shell structure with a collapsed hydrophobic core. In addition, we also compared ${ }^{1} \mathrm{H}$ NMR spectra of embelin loaded PEG-PCD lipopolymer in $\mathrm{CDCl}_{3}$ and $\mathrm{D}_{2} \mathrm{O}$. Although the specific peaks for embelin at 6 and $2.4 \mathrm{ppm}$ was observed when dissolved in $\mathrm{CDCl}_{3}$, they disappeared when they formed micelles in $\mathrm{D}_{2} \mathrm{O}$. 

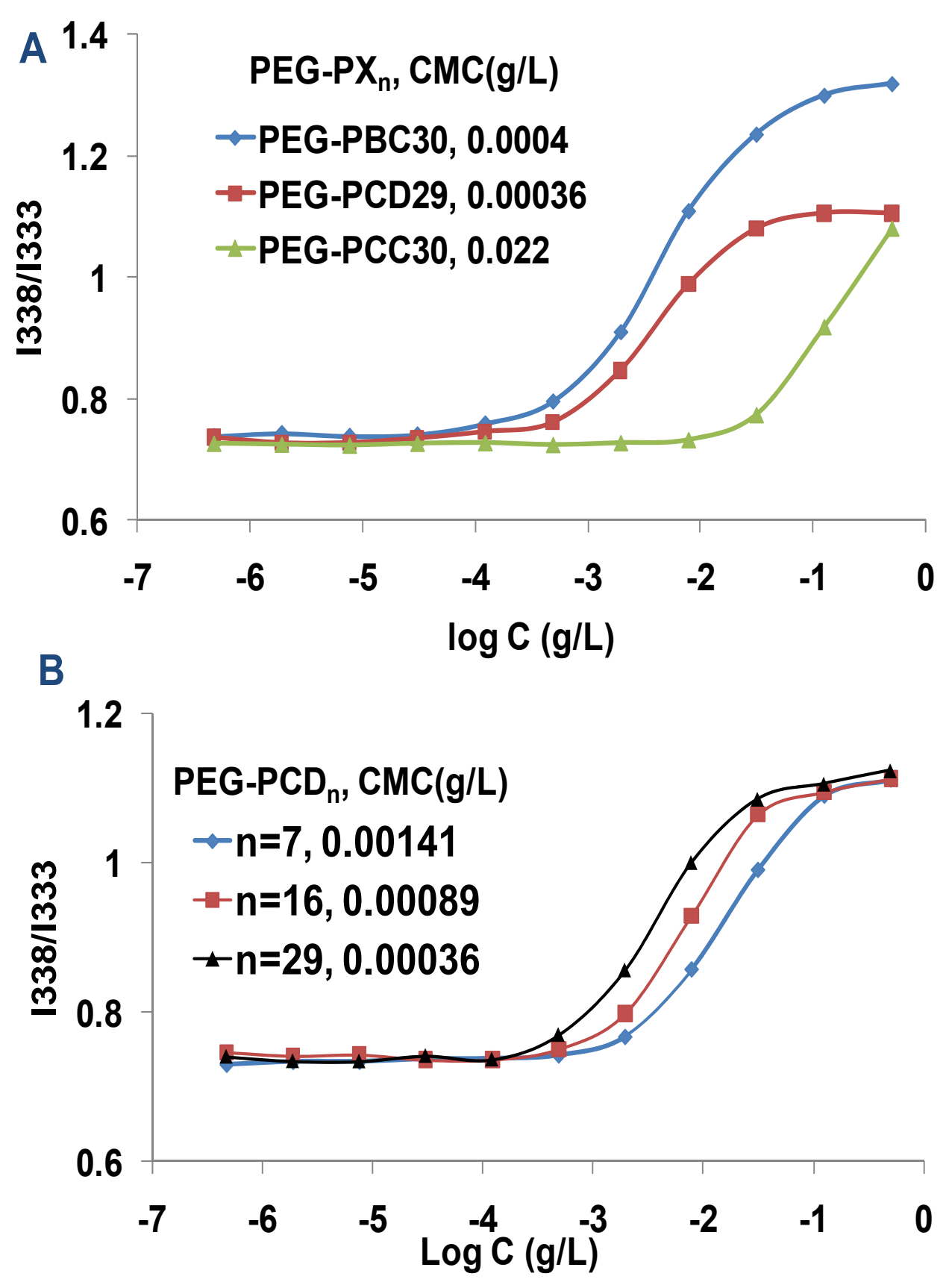

Figure 6.6 Determination of CMC values.

Plots of the intensity ratio $\mathrm{I}_{338} / \mathrm{I}_{333}$ from pyrene excitation spectra at $\lambda_{\mathrm{em}}=390 \mathrm{~nm}$ versus $\log C$ for (A) Polymer with different core structures: $\mathrm{PEG}_{114}-\mathrm{PBC}_{30}, \mathrm{PEG}_{114}-\mathrm{PCD}_{29}$, and $\mathrm{PEG}_{144}-\mathrm{PCC}_{30}$. (B) PEG-PCD polymers with different PCD core length: $\mathrm{n}=6,16$, and 29. 
A

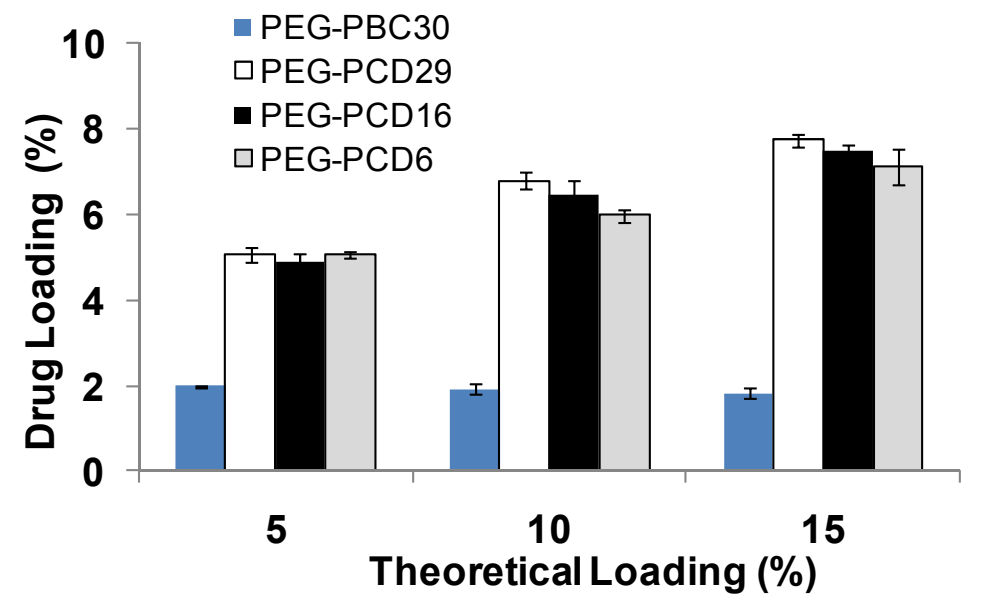

B

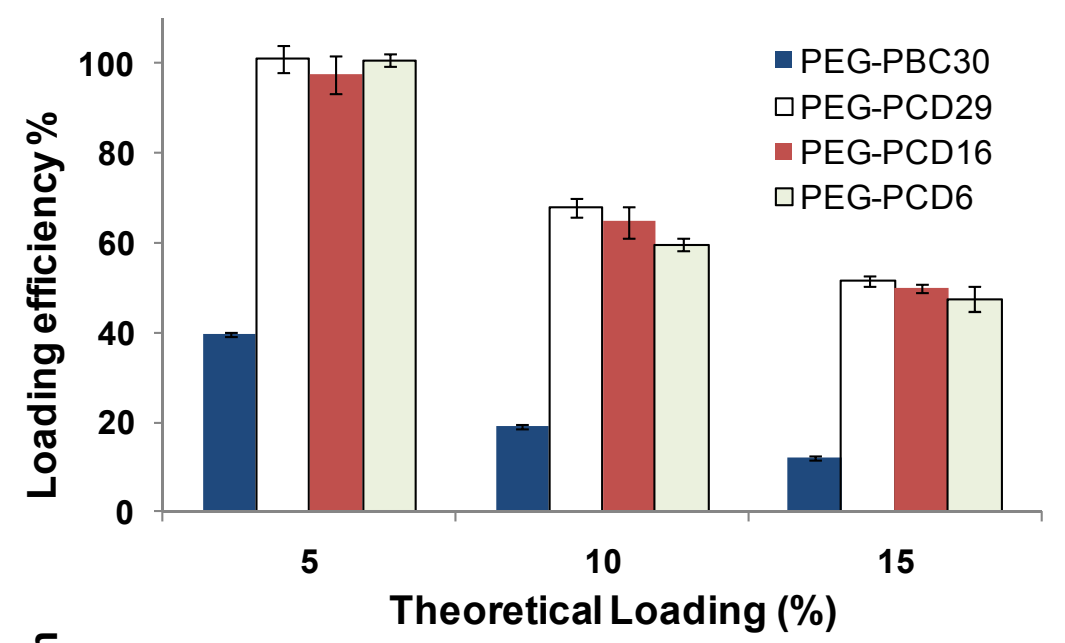

C

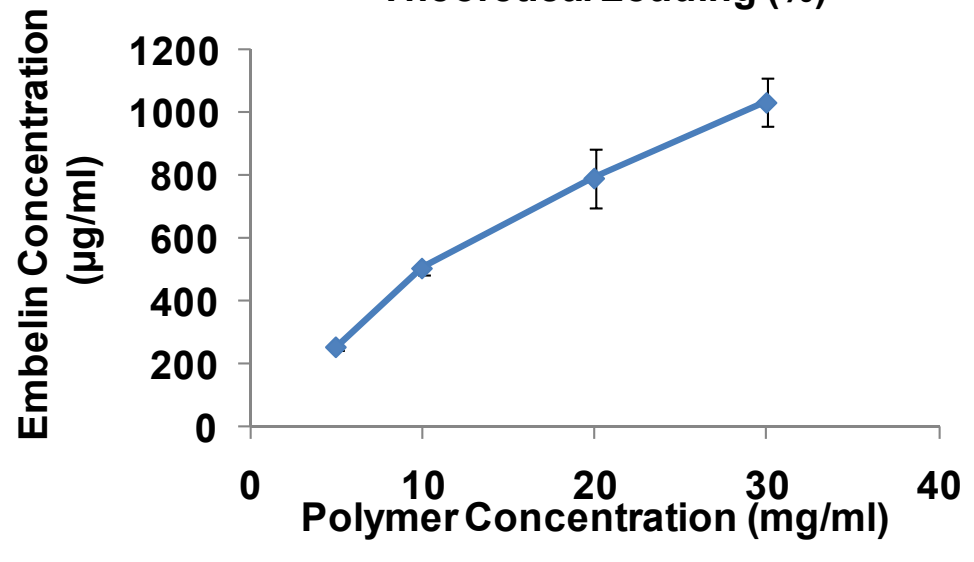

Figure 6.7 Optimization of embelin loaded micelle formulations.

(A) Effect of hydrophobic core structure and length on drug loading and (B) loading efficiency. Embelin loaded PEG-PBC and PEG-PCD micelles were prepared at 5, 10 and $15 \%$ of theoretical drug loading. (C) Encapsulation of embelin by PEG-PCD micelles as a function of polymer concentration. 


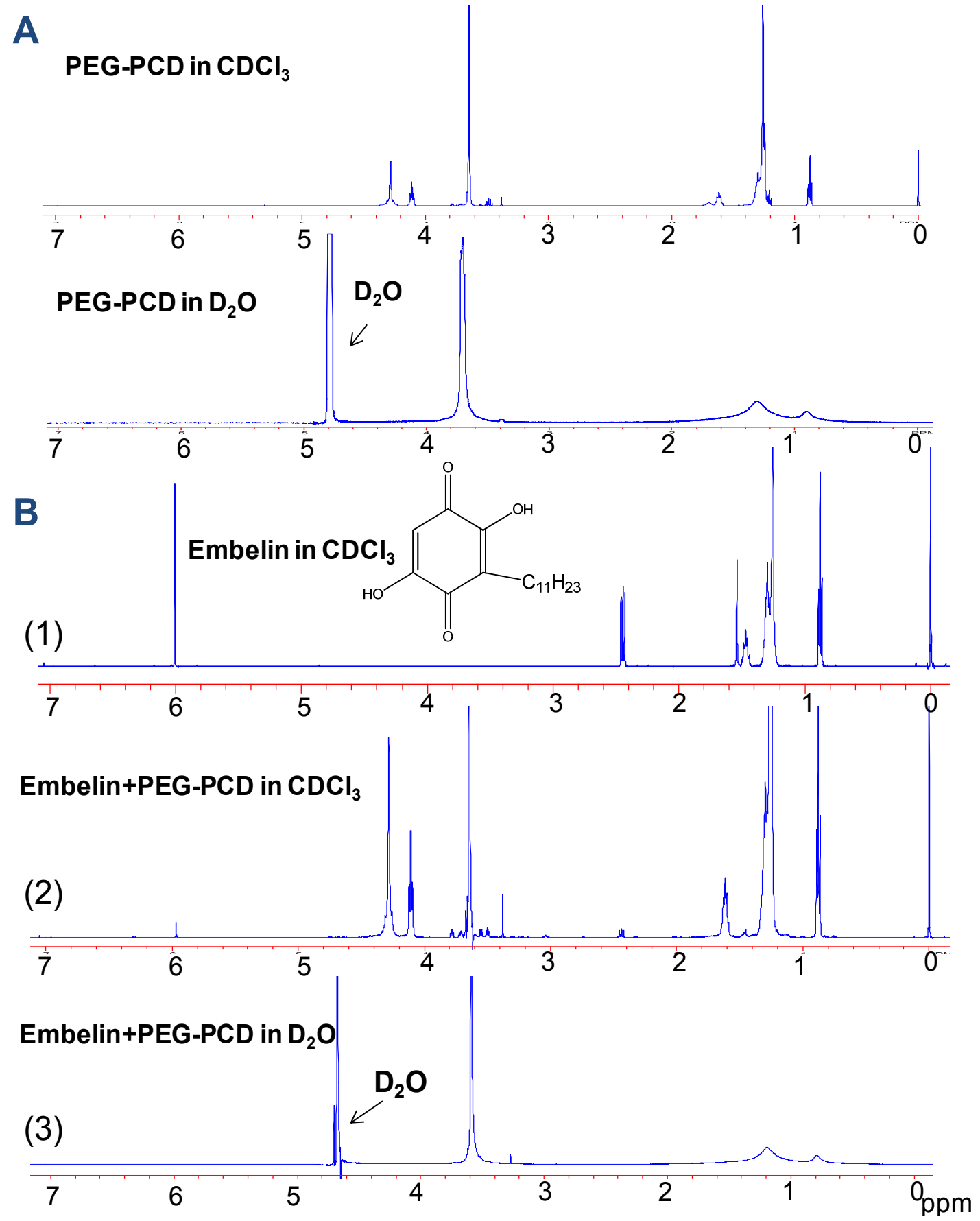

Figure 6.8 Core-shell structure formed by PEG-PCD lipopolymer.

(A) ${ }^{1} \mathrm{H}$ NMR of blank $\mathrm{PEG}_{114}-\mathrm{PCD}_{29}$ in $\mathrm{CDCl}_{3}$ and $\mathrm{D}_{2} \mathrm{O}$. (B) ${ }^{1} \mathrm{H}$ NMR of $\mathrm{PEG}_{114}-\mathrm{PCD}_{29}$ and emblin. (1) Free embelin dissolved in $\mathrm{CDCl}_{3}$. (2) embelin and $\mathrm{PEG}_{114}-\mathrm{PCD}_{29}$ dissolved in $\mathrm{CDCl}_{3}$ at embelin to polymer ratio of $3 \%$. (3) embelin loaded $\mathrm{PEG}_{114}-\mathrm{PCD}_{29}$ micelles in $\mathrm{D}_{2} \mathrm{O}$ at embelin to polymer ratio of $3 \%$. 
These results indicate that PEG-PCD lipopolymer will form micelles in water with a PEG hydrophilic shell and a hydrophobic core, and embelin is incorporated into the hydrophobic core.

\subsubsection{Physical appearance and particle size distribution}

The physical appearance of blank and drug loaded micelles were shown in Figure 6.9A. Both PEG-PCD and $\mathrm{PEG}_{114}-\mathrm{PBC}_{30}$ formed a translucent micelle solution, which was stable at the room temperature and showed no precipitation on storage at the room temperature for one week. In contrast, there was a milk-like suspension formation of $\mathrm{PEG}_{114}-\mathrm{PCC}_{30}$ copolymer micelles and precipitation was observed after overnight storage of these micelles. Also, the drug loaded micelles showed a pink color due to the solubilized embelin (EM). The color observed in $\mathrm{PEG}_{114}-\mathrm{PCD}_{29}$ micelles was darker than those of $\mathrm{PEG}_{114}-\mathrm{PBC}_{30}$ micelles prepared at same theoretical loading (5\%). And this was consistent with the high drug loading in $\mathrm{PEG}_{114}-\mathrm{PCD}_{29}$ micelles. We also determined the particle size and size distribution of blank and embelin loaded micelles by dynamic light scattering (Figure 6.9B). The blank and embelin loaded $\mathrm{PEG}_{114}-\mathrm{PCB}_{30}$ micelles had the mean particle size of $74 \mathrm{~nm}$ and $64 \mathrm{~nm}$, respectively. The particle size of blank and embelin loaded $\mathrm{PEG}_{114}-\mathrm{PCD}_{29}$ micelles was $129 \mathrm{~nm}$ and $110 \mathrm{~nm}$, respectively. There was no increase in the mean particle size after embelin loading. In contrast, micelles formed by $\mathrm{PEG}_{114}-\mathrm{PCC}_{30}$ showed a particle size of $204 \mathrm{~nm}$. We also determined the particle size of PEG-PCD with different PCD core length, the mean particle size were $129 \mathrm{~nm}$ for $\mathrm{PEG}_{114}-\mathrm{PCD}_{29}, 42.6 \mathrm{~nm}$ for $\mathrm{PEG}_{114}-\mathrm{PCD}_{16}$, and $25 \mathrm{~nm}$ for $\mathrm{PEG}_{114}-\mathrm{PCD}_{6}$, respectively.

\subsubsection{Morphology of micelles}

The morphology of micelles formed by PEG-PBC and PEG-PCD was determined by transmission electron micrography (TEM) (Figure 6.10). PEG-PCD formed micelles with different morphologies and sizes, depending on the ratio of hydrophobic core (Figure 6.10A-C). $\mathrm{PEG}_{114}-\mathrm{PBC}_{30}$ formed spherical micelles (Figure 6.10D). $\mathrm{PEG}_{114}-\mathrm{PCD}_{29}$ formed both spherical and cylindrical micelles with a diameter less than $50 \mathrm{~nm}$, and the cylindrical micelle was the dominant form. The contour length of the cylindrical micelles ranged from several hundred nanometers to several micrometers. However, $\mathrm{PEG}_{114^{-}}$ $\mathrm{PCD}_{16}$ and $\mathrm{PEG}_{114}-\mathrm{PCD}_{6}$, formed only spherical micelles. We also determined the morphology of embelin loaded micelles prepared from $\mathrm{PEG}_{114}-\mathrm{PCD}_{6}, \mathrm{PEG}_{114}-\mathrm{PCD}_{16}$, $\mathrm{PEG}_{114}-\mathrm{PCD}_{29}$, and $\mathrm{PEG}_{114}-\mathrm{PBC}_{30}$. In addition, the loading of embelin at 5\% in PEG$\mathrm{PCD}$ and $2 \%$ at $\mathrm{PEG}_{114}-\mathrm{PBC}_{30}$, had no effect on the morphology of micelles (data not shown).

\subsubsection{In vitro drug release}

The cumulative percentage of embelin released from PEG-PCD micelles with different PCD block length was shown in Figure 6.11A. Similarity factors $\left(f_{2}\right)$ were calculated to compare the drug release profiles of micelles with various hydrophobic core lengths. The similarity factors were $63(\mathrm{n}=6 \mathrm{Vs} 16), 78(\mathrm{n}=6 \mathrm{Vs} 29)$, and $74(\mathrm{n}=16$ Vs 29$)$, respectively. Therefore, these micelles showed similar release profiles, since the release 

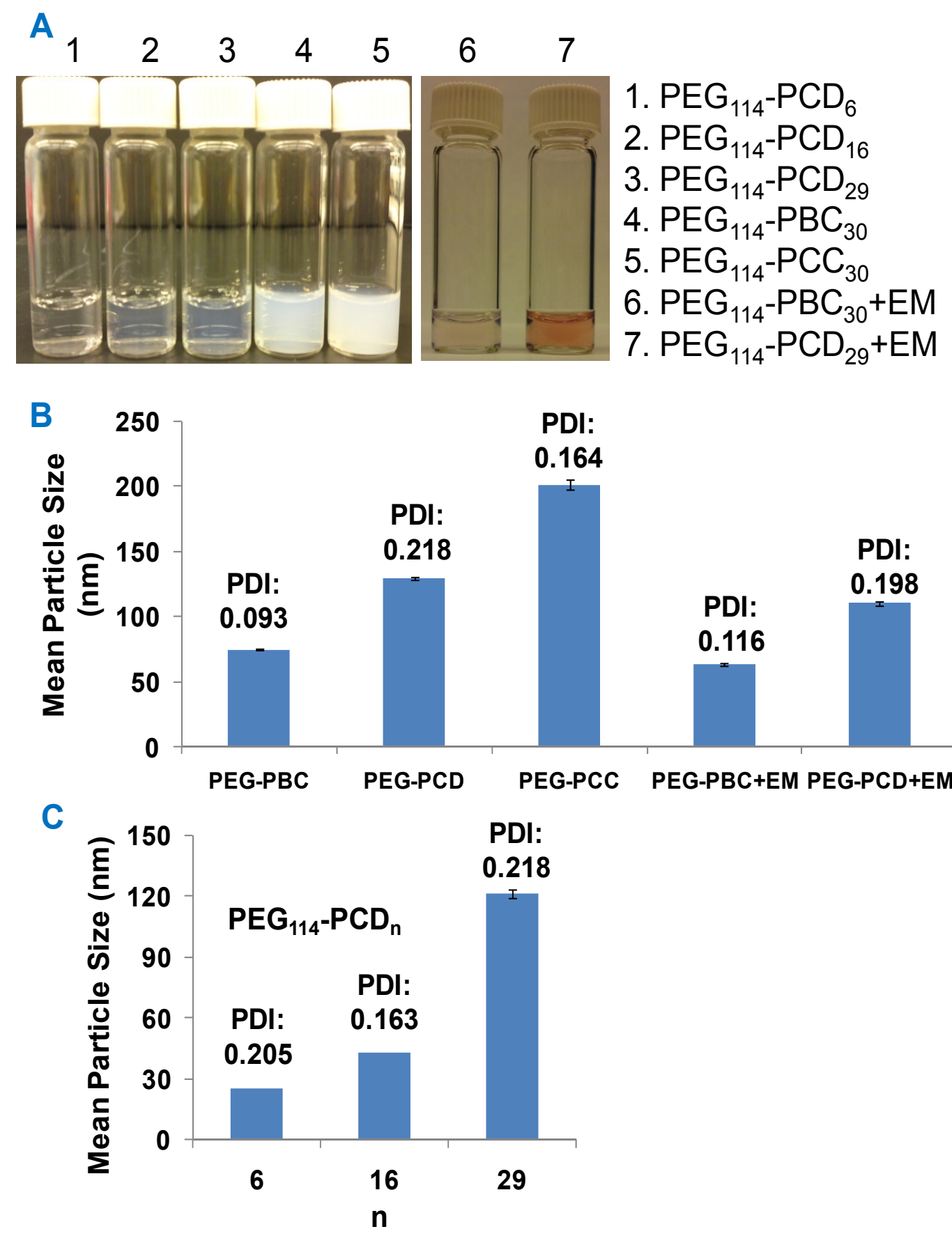

Figure 6.9 Physical appearance and particle size of micelles.

(A) Appearance of blank or embelin loaded micelles prepared from polymers with different core structure and length (polymer concentration $5 \mathrm{mg} / \mathrm{mL}$ ). (B) Mean particle size of blank or embelin loaded micelles prepared from $\mathrm{PEG}_{114}-\mathrm{PCD}_{29}, \mathrm{PEG}_{114}-\mathrm{PBC}_{30}$, and $\mathrm{PEG}_{114}-\mathrm{PCC}_{30}$. (C) Mean particle size of micelles prepared from PEG-PCD polymers with different PCD core length: $\mathrm{n}=6,16$, and 29 . 

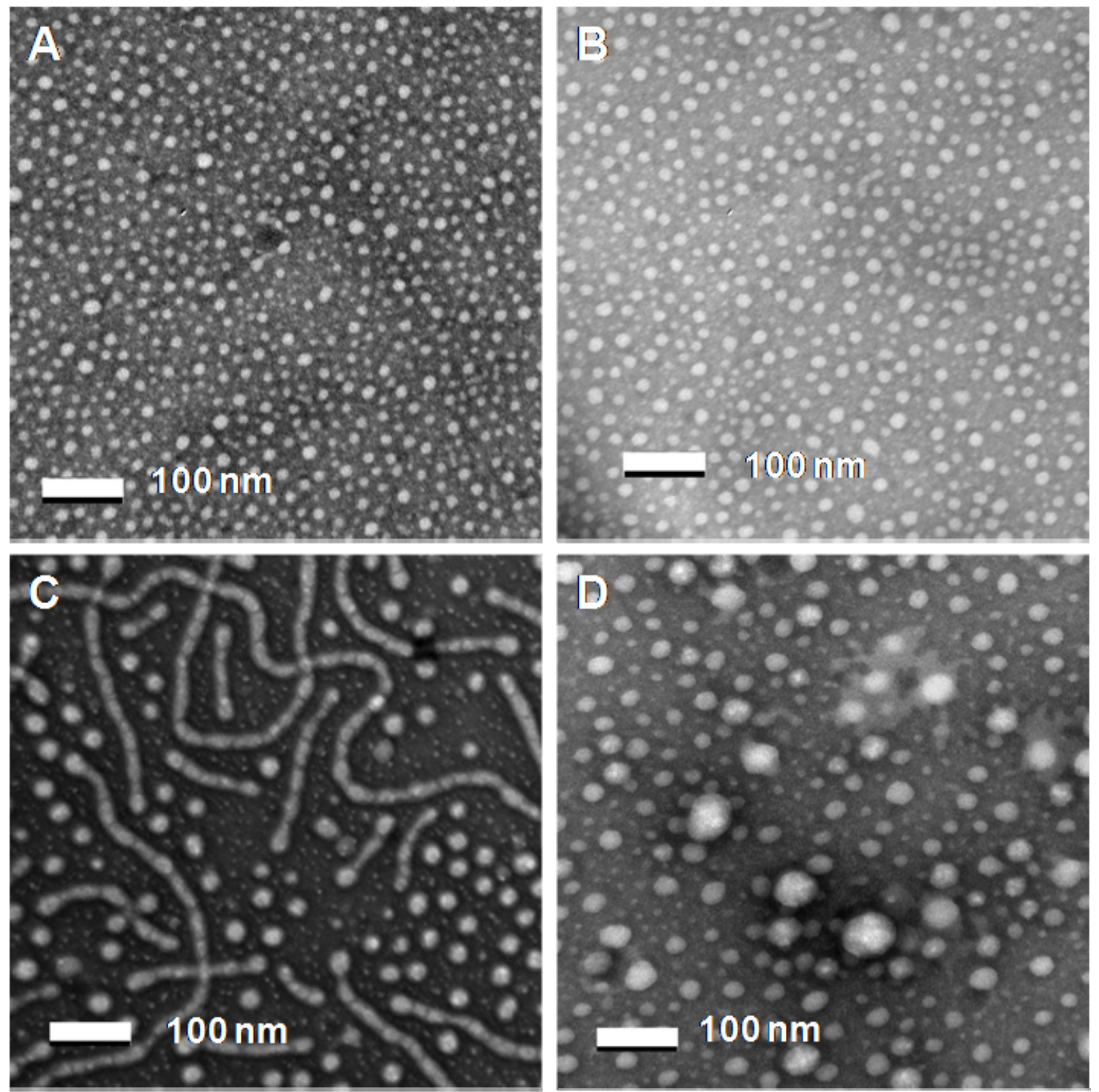

Figure 6.10 Transmission electron microscopy (TEM) of micelles.

Micelles were prepared from (A) $\mathrm{PEG}_{114}-\mathrm{PCD}_{6}$; (B) $\mathrm{PEG}_{114}-\mathrm{PCD}_{16}$; (C) $\mathrm{PEG}_{114}-\mathrm{PCD}_{29}$, and (D) $\mathrm{PEG}_{114}-\mathrm{PBC}_{30}$. 
profiles are considered as the same, when the similarity factors are greater than 50 . The release of embelin from PEG-PCD micelles was fit to a first-order process for the first 24 $\mathrm{h}$.The release half-life was around $12 \mathrm{~h}$. The effect of hydrophobic core on embelin release was also investigated (Figure 6.11B). The release of embelin from $\mathrm{PEG}_{114}-\mathrm{PCD}_{30}$ was significantly slower compared to $\mathrm{PEG}_{114}-\mathrm{PBC}_{30}$ with a similarity factor of 38 . In addition, the embelin release half-life from $\mathrm{PEG}_{114}-\mathrm{PBC}_{30}$ micelles was $4 \mathrm{~h}$, which was much shorter than that of $\mathrm{PEG}_{114}-\mathrm{PCD}_{30}$.

\subsubsection{Bio-evaluation of drug loaded micelles}

The anticancer activity of embelin loaded PEG-PCD micelles was determined in C4-2 prostate cancer cells. As shown in Figure 6.12, while blank $\mathrm{PEG}_{114}-\mathrm{PCD}_{29}$ polymer showed negligible effect on cell proliferation at polymer concentration up to $0.3 \mathrm{mg} / \mathrm{ml}$, embelin loaded $\mathrm{PEG}_{114}-\mathrm{PCD}_{29}$ micelles showed significant inhibition of $\mathrm{C} 4-2$ cell proliferation in a dose dependent manner, with the $\mathrm{IC}_{50}$ of $10 \mu \mathrm{M}$. The free drug dissolved in DMSO showed similar anticancer effects to micelle formulated embelin (data not shown). No direct comparison can be made with the results of embelin formulated in micelles and dissolved in DMSO, as DMSO is known to kill tumor cells and may not be suitable for in vivo applications. However, this finding is significant, since the application of polymeric micelles could avoid the use of toxic solubilzing agents such as DMSO, Cremophore EL and Tween 80.

\subsection{Discussion}

Polymeric micelles can serve as a vehicle, which self-assemble into a core-shell structure with a hydrophobic core capable of loading hydrophobic drugs $[350,351]$. The stealth properties associated with hydrophilic PEG corona of micelles prevent their aggregation, restrict plasma protein adsorption, prevent recognition by the reticuloendothelial system (RES), and minimize their rapid elimination from the bloodstream. The small size of micelles ensures their accumulation preferentially in the tumor via EPR effects [323, 352]. However, successful micellar drug delivery into tumor requires the micelles remain stable during blood circulation upon systemic administration. The stability of micelles could be enhanced by crosslinking of the shell, core-shell interface, or the core of the micelles [353-355]. However, the application of chemical crosslinked micelles is limited due to the complexity of crosslinking process, possible effects on the structure and properties of loaded drugs, and problems associated with drug release. Alternatively, the stability of micelles could also be improved by engineering the structure of hydrophobic block by introducing aromatic moieties [356, 357].

We have successfully synthesized PEG-PCD lipopolymer with two different approaches (Figure 6.1). However, the first approach was selected due to the relative simplicity in the synthesis process, which is critical for its application. In this synthesis approach, PEG$\mathrm{PBC}$ was synthesized by ring-opening polymerization of $\mathrm{MBC}$ in the presence of $\mathrm{PEG}$ carrying a hydroxyl end group with one of the following catalysts: DBU, $\mathrm{Sn}(\text { oct })_{2}$, and $\mathrm{Et}_{2} \mathrm{Zn}$ (Table 6.1). Relatively lower reaction efficiency was observed with $\mathrm{Sn}(\mathrm{oct})_{2}$, and 

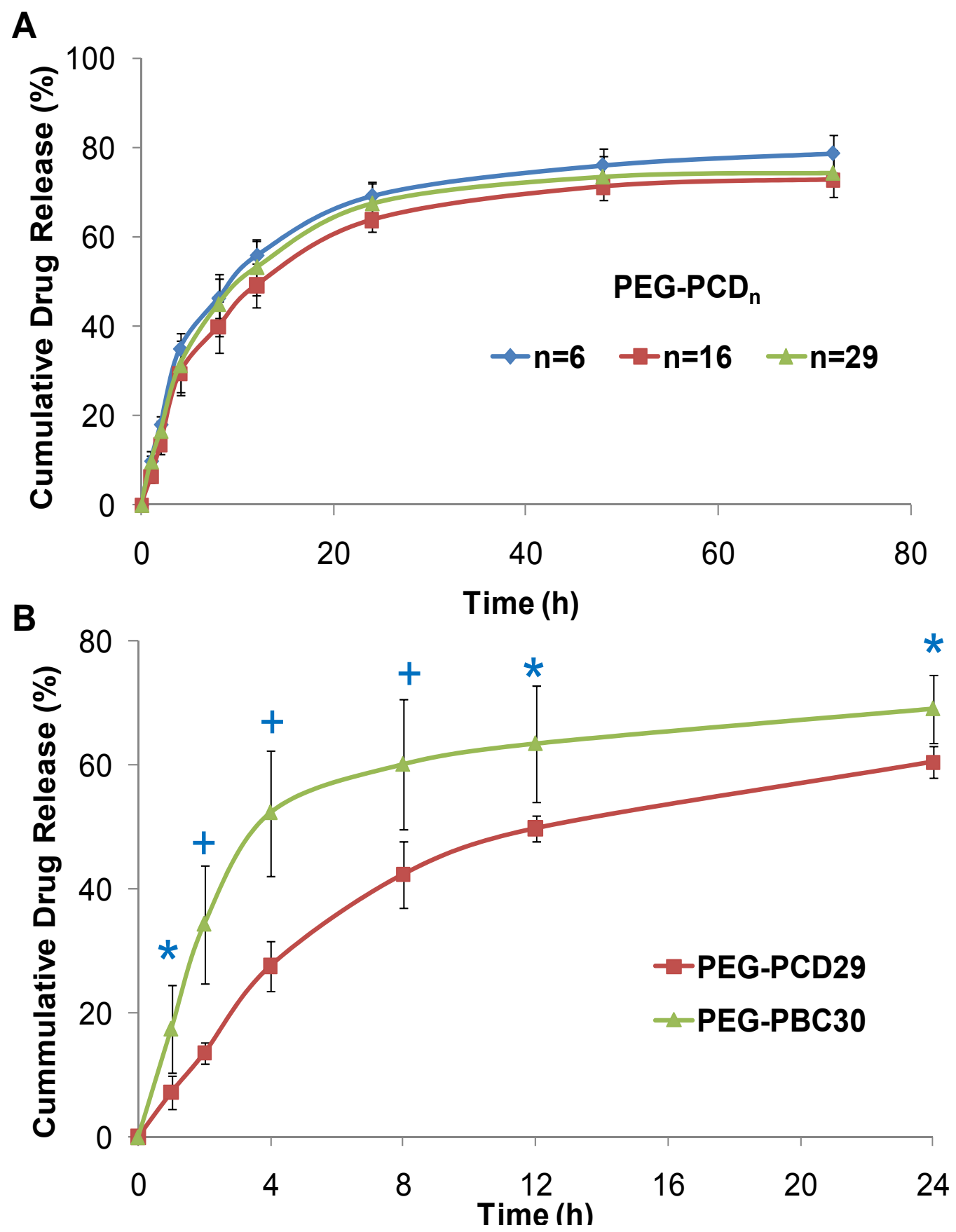

Figure 6.11 In vitro release of embelin from polymeric micelles.

(A) Release profiles of PEG-PCD micelles with different PCD core lengths at drug loading of 5\%. (B) Release profiles of $\mathrm{PEG}_{114}-\mathrm{PCD}_{29}$, and $\mathrm{PEG}_{14}-\mathrm{PBC}_{30}$ micelles at drug loading of $2 \%$. *, $\mathrm{P}<0.05,+, \mathrm{P}<0.01$, compared with $\mathrm{PEG}_{114}-\mathrm{PBC}_{30}$ micelles using Student's unpaired $t$ test. Results were expressed as Mean $\pm \operatorname{SD}(n=3)$. 


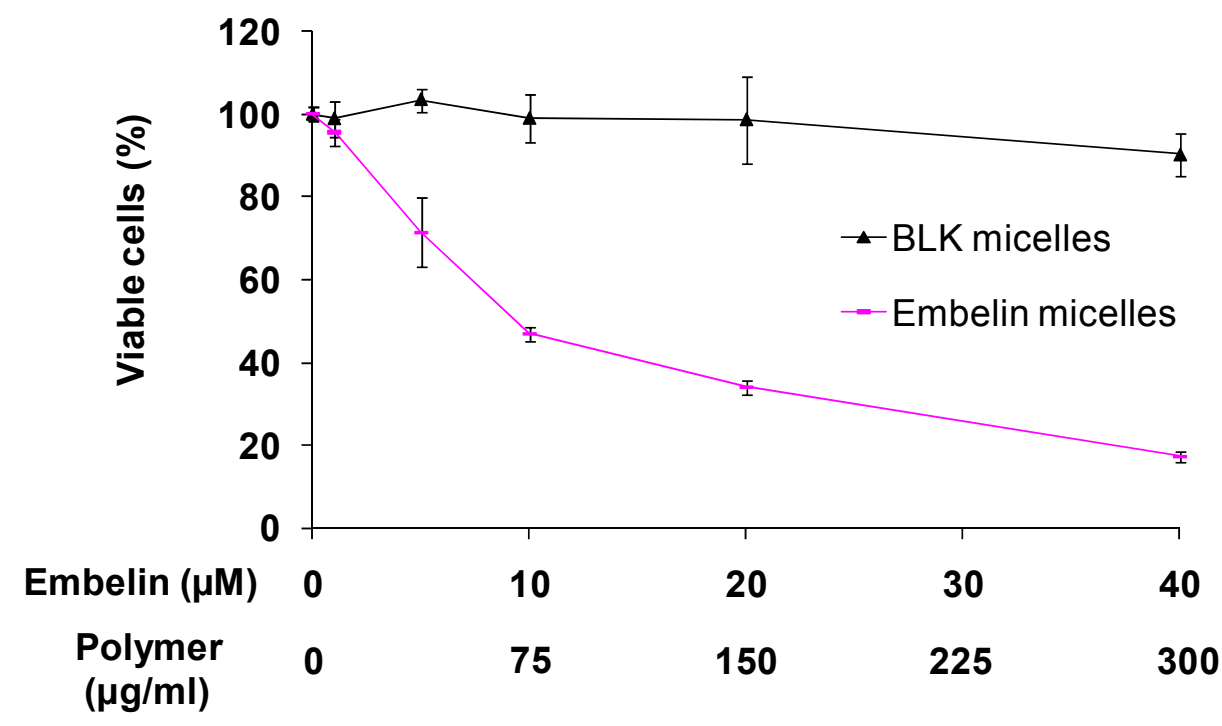

Figure 6.12 Inhibition of C4-2 cell proliferation by PEG-PCD blank micelle and micelle formulated embelin.

Lipopolymer micelles were prepared with $\mathrm{PEG}_{114}-\mathrm{PCD}_{29}$. The weight ratio of embelin and polymer was $4 \%$ (embelin/polymer). Blank micelles with same polymer concentration were served as control. Cell viability was determined by MTT assay at $72 \mathrm{~h}$ post treatment. Results were expressed as Mean \pm SD $(n=4)$. 
$\mathrm{Et}_{2} \mathrm{Zn}$, this is due to the high reaction temperature and long reaction time required for these two catalysts, which is usually associated with the potential polymer degradation due to transesterification or backbiting side reaction $[356,358]$. In addition, the use of these metallic catalysts is also associated with safety issues due to the presence of trace metal residue in the polymer. Therefore, DBU was used in the current study, which is purely organic and suitable for the synthesis of biomaterials. Besides, the reaction was carried out in solution and at room temperature, which is superior to bulk reaction that usually results in broad polydispersity due to the inefficient mixing of reactants. To optimize the reaction time, molecular weight of the polymer was monitored during the reaction. We found that the reaction was almost completed after $3 \mathrm{~h}$ and the DP of polymerization decreased after reaction for $24 \mathrm{~h}$. This may be due to the occurring of transesterification of polymer which becomes dominant when monomer is consumed [358]. Therefore, the reaction time was set to $3 \mathrm{~h}$ to achieve optimal polymerization efficiency and polydispersity. Subsequently, the benzyl groups in the PEG-PBC were removed via hydrogenation to expose $\mathrm{COOH}$ function group. This reaction was also carried out under at the room temperature to avoid the potential degradation of polymer backbone, while still maintaining the high reaction efficiency to completely remove benzyl groups. The dodecanol was conjugated to the $\mathrm{COOH}$ groups in the polymer backbone through EDC/HOBT coupling chemistry with conjugation efficiency of around $95 \%$. This is much higher than the previous report, where less than $70 \%$ of conjugation efficiency was achieved in conjugating fatty acids to poly(ethylene glycol)-block-poly( $\beta$ benzyl-L-aspartate) through a link molecule [359]. Because of the commercial availability of starting materials, simple synthesis procedure, and high reaction efficiency, this synthesis procedure will have a broad application in the drug delivery and bioengineering.

PEG-PCD lipopolymer synthesized in the current study has the polycarbonate backbone and dodecanol was conjugated to this backbone with an ester bond. Therefore, this is a biodegradable polymer, which is expected to degrade in vivo through hydrolysis of the ester linkages. The hydrolysis of polycarbonate backbone was facilitated at the presence of esterase such as lipase or cholesterol esterase [360, 361]. In addition, the final degradation product was $\mathrm{CO}_{2}$, dual alcohol, dodecanol, and $\mathrm{PEG}_{5000}$, which have less effect on $\mathrm{pH}$ change in vivo compared to poly(D,L-lactide) and thus are less likely to cause inflammation [362]. These degradation products are non-toxic and could be eliminated from the body.

PEG-PCD lipopolymer has multiple dodecanol lipid chains attached to the polycarbonate backbone, which mimics the structure of interface crosslinked PEG-lipids (Figure 6.13) and could enhance the hydrophobic interaction among the hydrophobic cores. Because the crosslinking of micelles are known to improve stability, it is not surprising to observe significantly reduced CMC values for PEG-PCD lipopolymer compared to conventional PEG-lipid conjugates. Due to its low CMC value $\left(\sim 10^{-8} \mathrm{M}\right)$, the concentration of PEGPCD in the bloodstream is expected to be much higher than the CMC value of PEG-PCD after systemic administration, which indicates its good in vivo stability. Therefore, PEGPCD lipopolymer micelles can serve as an alternative to cross-linked PEG-lipid micelles for in vivo drug delivery. 

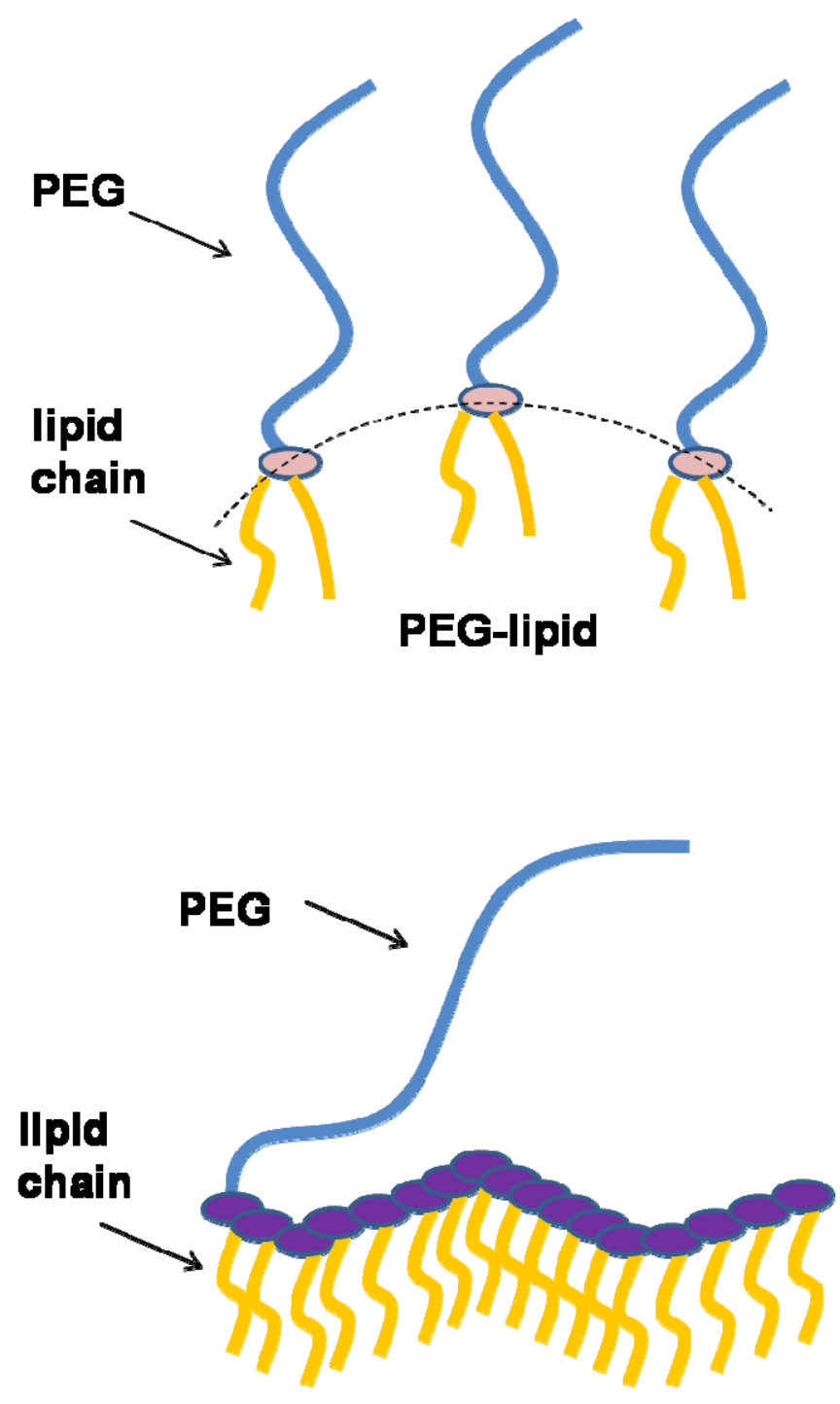

lipopolymer PEG-PCD

Figure 6.13 Structure of PEG-lipid and lipopolymer PEG-PCD. 
Among PEG-PCD series, we found that the CMC decreased with increasing the number of lipid in the hydrophobic core (Figure 6.6B). This is due to the increased length of hydrophobic block, which is known to enhance the hydrophobic interaction among the core block [363]. The effects of core structure were also investigated and the results showed that the CMC of PEG-PCD or PEG-PBC was around 50 folds lower than corresponding PEG-PCC (Figure 6.6A). This is because of the presence of hydrophobic aromatic rings in PEG-PBC and lipid chains in PEG-PCD, which will all facilitate the hydrophobic interaction within the core of micelles and promote the self-assembly of polymeric micelles. However, PEG-PCC has a higher CMC due to the presence of $\mathrm{COOH}$ group which significantly reduced the hydrophobicity of the core block. Comparable CMC values were observed for PEG-PBC and PEG-PCD, though the structure of $\mathrm{PBC}$ and $\mathrm{PCD}$ are significantly different (Figure 6.6A). Since the backbone is the same for PEG-PBC and PEG-PCD, the close similarity in CMC values are due to comparable contributions by the pedant phenyl rings in the $\mathrm{PBC}$ and the pedant dodecanol carbon chain in PCD to the core block hydrophobicity.

Spherical, cylindrical, and vesicular assemblies are three commonly observed morphologies of micelles prepared from amphiphilic block copolymers. Recently, interest has shifted from spherical to cylindrical micelles, due to their longer systemic circulation time compared to spherical micelles [364-366]. The cylindrical micelles prepared from poly(ethylene glycol)-b-poly(caprolactone) (PEG-PCL) showed significantly longer circulation time than that of spherical micelles prepared from the same polymer and was persistent up to several days after intravenous injection [364]. In addition, paclitaxel loaded cylindrical micelles were more effective to induce apoptosis and reduce the tumor size in tumor bearing mice $[365,366]$. Several approaches have been investigated to control the morphology of micelles, including kinetic control [367], the use of interfacial instability [368], aromatic oxidation [369], and the use of nanopore extruder [370]. The most important parameter that determines the morphology and architecture of micelles is the copolymer composition and volume ratio between insoluble and soluble blocks. Sumeet et al. have studied the effect of molecular size and composition using poly(ethylene glycol)-b-poly(1,2-butadiene)(PEG-PB) as a model diblock copolymer. These authors found that PEG-PB form spheres, cylinders, and bilayers in water, sequentially, with decreased ratio of PEG block [371]. This could explain the transition from spherical to cylindrical micelles with the increased hydrophobic length of PEG-PCD. In the current study, the use of PEG-PCD 29 with a PEG ratio of 0.37 produced cylindrical micelles, while $\mathrm{PEG}-\mathrm{PCD}_{6}$ (PEG ratio: 0.49$)$ and PEG$\mathrm{PCD}_{16}$ (PEG ratio: 0.72) produced only spherical micelles. Although PEG-PCD ${ }_{29}$ formed cylindrical micelles, PEG-PBC 30 , a polymer with similar hydrophobic core length did not form cylindrical micelles but spherical micelles (Figure 6.10). This indicates that not only the PEG ratio but also the structure of hydrophobic core determines the morphology of micelles. Thus the morphology of micelles can be controlled by changing the length and structure of hydrophobic core of polymer. The preparation of micelles of different morphologies will help us to understand the effect of micelles morphology and their in vivo biodistribution and bioactivities. 
The compatibility of core-forming block and the drug to be loaded is critical for drug loading capacity [344]. One objective of this study was to design an engineered polymeric micelle for improved solubilization and delivery of embelin based on the polymer-drug compatibility. PEG-PCD showed significantly high embelin loading capacity compared with PEG-PBC (Figure 6.7), although these two polymers have similar CMC value and comparable thermodynamic stability. The compatibility is highly dependent on the three-dimensional arrangement and conformation of different groups in the structure of drug and polymer core [357]. Figure 6.14 illustrates the structure of embelin and the repeating units of PEG-PBC and PEG-PCD hydrophobic core block. In contrast to PEG-PBC, the structure of the repeating unit of PEG-PCD is similar to that of embelin. A similar finding was reported previously, where the attachment of cholesterol to the poly(caprolactone) block of a poly(ethylene glycol)-b-poly(caprolactone) copolymer significantly improved the loading of cucurbitacin I, which is similar to cholesterol in the structure [357]. Our previous data also showed that the introduction of carbonate moiety into a PEG-PLA copolymer significantly increased the micellar solubility and loading of bicalutamide into micelles [344]. In this study, we also found that PEG-PCD lipopolymers with different core block length (repeating unit number ranges from 6 to 29) did not show significant difference in the drug loading (Figure 6.7). Although an increased drug loading efficiency was observed with increased length of PCL blocks in PEG-PCL polymer [372], this may not be applicable for PEG-PCD, which has a highly branched structure in contrast to the linear structure of PEG-PCL. In fact, a good drug loading was observed in PEG-lipid conjugates with much shorter hydrophobic core length [337], indicating that the further increase in hydrophobic length might not be able to improve the drug loading of lipopolymers with similar structure as PEG-PCD. Similarly, we also observed there was no significant difference in the in vitro drug release profiles among these PEG-PCD polymers with different block lengths (Figure 6.11A). The drug release profiles of polymeric micelles are influenced by several factors including strength of the interaction between drug and hydrophobic core, physical state of the core, the amount of drug loaded, the length of hydrophobic core, and the location of drug inside the micelle core [363]. In addition, the highly branched structure in the hydrophobic core of PEG-PCD may have contributed to the observation in this study. We also found that the release of embelin from PEG-PCD was much slower than that from PEG-PBC (Figure 6.11B). This might due to the fact that the interaction between PEGPCD and embelin was stronger than those of PEG-PBC and embelin.

\subsection{Conclusion}

We have successfully synthesized a novel lipopolymer poly(ethylene glycol)-blockpoly(2-methyl-2-carboxyl-propylene carbonate-graft-dodecanol) (PEG-PCD) for delivery of a hydrophobic anticancer drug, embelin. PEG-PCD lipopolymer could form micelles in water through self-assembly and significantly improved embelin solubility by loading drugs inside its hydrophobic core. The drug loading and encapsulation efficiency were dependent on the length and structure of polymer hydrophobic core block. The CMC of PEG-PCD micelles was around $10^{-8} \mathrm{M}$ and decreased with increasing the length of hydrophobic block, indicating good thermodynamic stability of PEG-PCD micelles. 


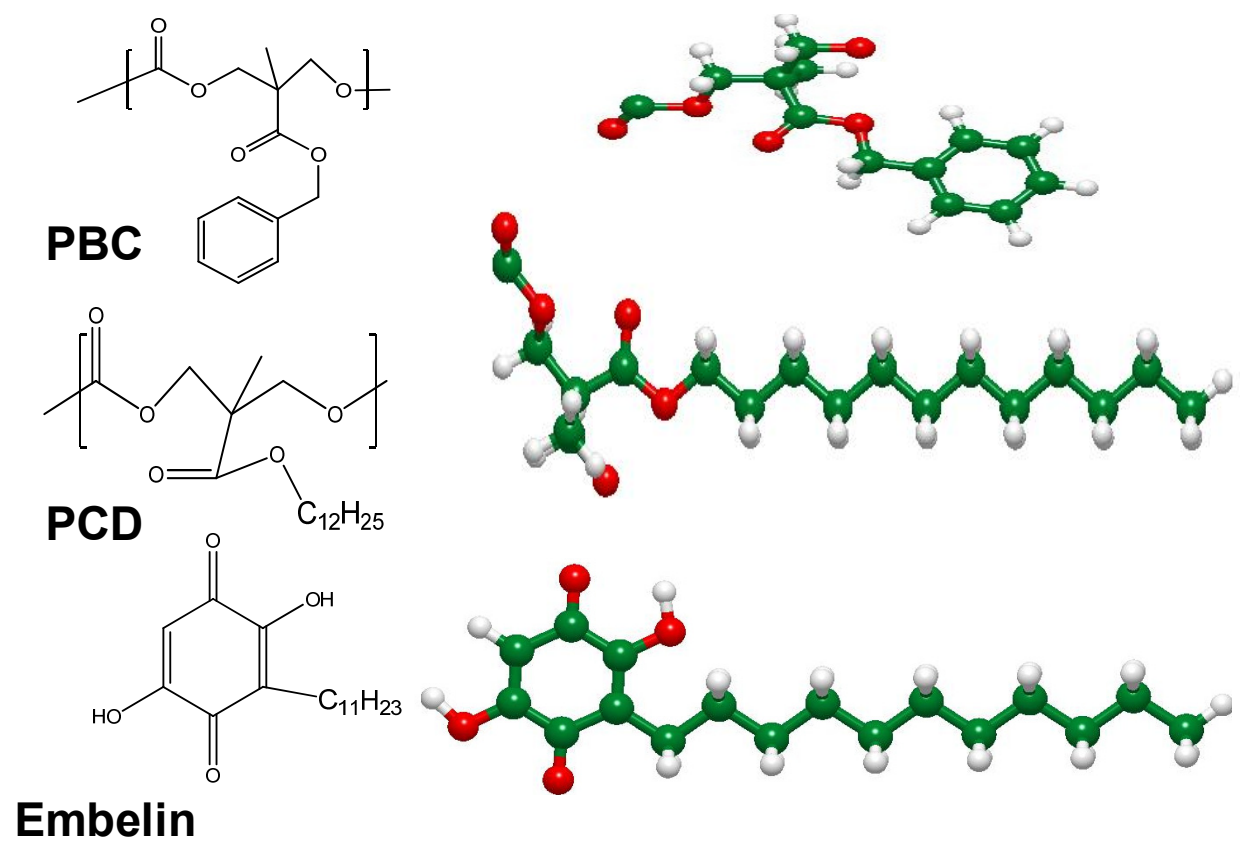

Figure 6.14 Structure of embelin and repeating unit of hydrophobic core. 
The particle size and morphology of micelles were also dependent on the structure and length of hydrophobic core. Embelin loaded PEG-PCD micelles showed significant inhibition of C4-2 prostate cancer cell proliferation in a dose dependent manner, while no obvious cellular toxicity was observed with blank micelles themselves. Thus, these lipopolymer have the potential to be used as vehicles for anticancer hydrophobic drug delivery. 


\section{CHAPTER 7. PACLITAXEL AND LAPATINIB LOADED LIPOPOLYMER MICELLES OVERCOME MULTIPLE DRUG RESISTANCE IN PROSTATE CANCER}

\subsection{Introduction}

Prostate cancer is the second common male cancer in the United States. More than 186,000 American men learn are diagnosed to have prostate cancer each year (http://www.cancer.gov/cancertopics/wyntk/prostate). Although the majority of patients respond well to androgen ablation therapy, chemotherapy, and radiotherapy at the beginning, many patients relapse over time and become resistant to chemotherapy [373, 374]. This is mainly due to the over-expression of multiple drug resistant (MDR) transporters in prostate cancer cells (Figure 7.1). These transporters, such as P-gp, breast cancer resistance protein (BCRP), and multiple drug resistance protein (MRP), increase drug efflux and reduce drug accumulation in tumor cells $[375,376]$.The prognosis of patients with MDR cancer is poor, due to the lack of effective clinical interventions. Thus, there is an urgent need for effective therapies for MDR prostate cancers. Several strategies have been used to overcome MDR, including developing of novel anticancer agents [348], using novel delivery systems, such as liposomes [377], solid lipid nanoparticles [327], polymer-drug conjugates [324], and the use of MDR transporter inhibitor [378].

Lapatinib, a dual inhibitor of epidermal growth factor receptor (EGFR) and human epidermal growth factor receptor 2 (Her-2) tyrosine kinases, was approved by the FDA for the treatment of advanced breast cancers [379]. Studies indicate that lapatinib can inhibit the function of ATP-binding cassette (ABC) transporters, and thus sensitize MDR cancer cells to chemotherapeutic agents (Figure 7.1) [380-382]. Lapatinib has also been reported to decrease the percentage of cancer stem cells and improved the long term survival of patients [383]. Therefore, the combination of lapatinib and paclitaxel provides a new regimen for treating MDR cancers [380].

Polymeric micelle is a recently developed drug delivery system and prepared from amphiphilic diblock copolymers. It forms a core-shell structure by self-assembly. The hydrophobic core of micelles is capable of loading hydrophobic drugs, and thus avoids the use of toxic solubilizing agents in the delivery of poorly soluble anticancer drugs $[350,351]$. The stealth properties associated with hydrophilic PEG corona of micelles prevent their aggregation, restrict plasma protein adsorption, prevent recognition by the reticuloendothelial system (RES), and minimize rapid elimination from the bloodstream. The small size of micelles ensures their accumulation preferentially in the tumor via EPR effects [323, 352]. In order to targeted deliver drugs into tumors, the micelles need to be stable in the blood circulation. The stability of micelles can be improved through the engineering of the hydrophobic core. In our previous study, we designed a lipopolymer poly(ethylene glycol)-block-poly(2-methyl-2-carboxyl-propylene carbonate-graftdodecanol) for micelle drug delivery. This lipopolymer showed significantly improved stability and good drug loading for many poorly soluble drugs [384] . 


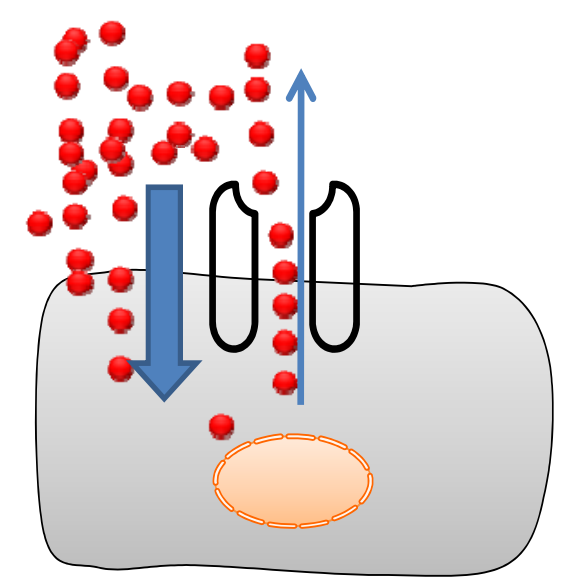

Resistance
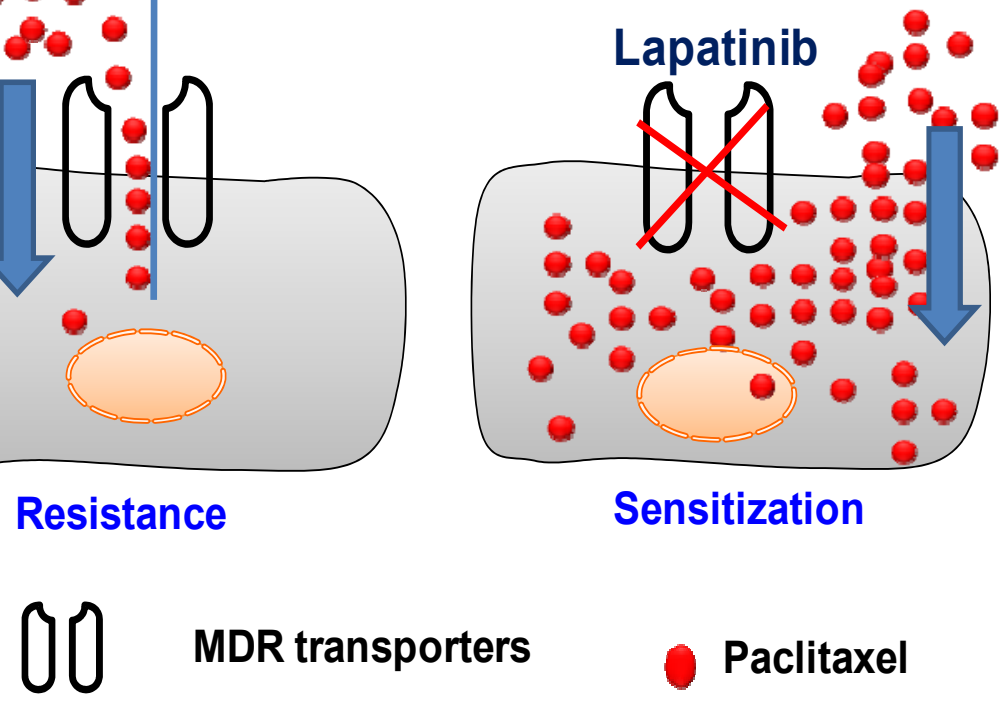

Figure 7.1 Paclitaxel and lapatinib combination for treating MDR cancer. 
In the current study, we used lipopolymer PEG-PCD micelles to formulate lapatinib and paclitaxel. Firstly, we optimized the micelle formulation and characterized the formulation with analytical and biophysical methods. Then, the anticancer effects of lapatinib and paclitaxel micelle formulations were determined with in vitro cell-based assays, focusing on their effects on overcoming MDR in prostate cancers. We also tested their abilities to inhibit of MDR growth in vivo with athymic mice xenograft tumor models established with the MDR prostate cancer cells.

\subsection{Materials and Methods}

\subsubsection{Materials}

Hydroxybenzotriazole (HOBT), 1-ethyl-3-(3-dimethylaminopropyl)carbodiimide (EDC), dodecanol, triethylamine (TEA), 1,8-diazabicyclo[5.4.0] undec-7-ene (DBU), 2,2bis(hydroxymethyl) propionic acid, methoxy poly(ethylene glycol) (mPEG, Mn = 5000, PDI $=1.03$ ) and all other reagents were purchased from Sigma Aldrich (St. Louis, MO) and used as received. DU145-TXR cells were provided by Professor Evan T. Keller of the University of Michigan.

7.2.2. Synthesis of lipopolymer poly(ethylene glycol)-block-poly(2-methyl-2carboxyl-propylene carbonate-graft-dodecanol) (PEG-PCD)

Lipopolymer PEG-PCD was synthesized as described by Li et al. [384]. Firstly, poly(ethylene glycol)-block-poly(2-methyl-2-benzoxycarbonyl-propylene carbonate) (PEG-PBC) was synthesized through a ring-opening polymerization from monomer MBC, using $\mathrm{mPEG}$ as macro imitator and DBU as a catalyst. Then, protective benzyl groups in PEG-PBC was removed by hydrogenation to get poly(ethylene glycol)-blockpoly(2-methyl-2-carboxyl-propylene carbonate) (PEG-PCC). Finally, lipopolymer PEGPCD was obtained after conjugation of dodecanol lipid pendant groups to PEG-PCC.

Lipopolymers and intermediate polymers were characterized with following methods. (1) Nuclear magnetic resonance (NMR). ${ }^{1} \mathrm{H}$ NMR spectra were recorded on Varian (500 $\mathrm{MHz})$ using deuterated chloroform $\left(\mathrm{CDCl}_{3}\right)$ as a solvent unless otherwise noted. The chemical shifts were calibrated using tetramethylsilane as an internal reference and given in parts per million. (2) Gel permeation chromatography (GPC). The weight $\left(\mathrm{M}_{\mathrm{w}}\right)$ and number $\left(\mathrm{M}_{\mathrm{n}}\right)$ average molecular weight and polydispersity index (PDI) of synthesized polymers were determined by a Waters GPC system equipped with a GPC column (AM Gel $10^{3} / 5$ ) and a differential refractive index detector. THF was used as an eluent at a flow rate of $1 \mathrm{~mL} / \mathrm{min}$. A series of narrow polystyrene standards $(700-40,000 \mathrm{~g} / \mathrm{mol})$ were used for calibration. 
7.2.3. Preparation and characterization of lipopolymer micelles

\subsubsection{Preparation of lipopolymeric micelles}

Lipopolymer micelles were prepared with a film dispersion method as previously reported with some modifications [348]. Briefly, $30 \mathrm{mg}$ of polymers and given amount of drugs were dissolved in $0.5 \mathrm{~mL} \mathrm{CH}_{2} \mathrm{Cl}_{2}$, and then the solvent was removed under reduced pressure. The resulting film was hydrated in $3 \mathrm{ml}$ saline $(0.9 \%)$ and sonicated for $1 \mathrm{~min}$. Then, the residue free drug was removed by centrifugation at 12,000 rpm for $5 \mathrm{~min}$. The supernatant was filtered using a $0.22 \mu \mathrm{M}$ filter.

\subsubsection{Drug loading and encapsulation efficiency}

To determine drug loading, $10 \mu \mathrm{L}$ of drug loaded micelle solution was diluted with acetonitrile. Then drug concentration was determined using a reverse phase high performance liquid chromatography (RP-HPLC, Waters, Milford, MA) with UV detector at $227 \mathrm{~nm}$. A C18 column ( $250 \mathrm{~mm} 4.6 \mathrm{~mm}$, Alltech, Deerfield, IL) was used. The mobile phase was composed of 50:50 V/V water and acetonitrile at $1 \mathrm{~mL} / \mathrm{min}$. Lapatinib and Paclitaxel concentration was calculated based on peak area.

\subsubsection{Measurement of particle size}

The particle size distribution of micelles was determined by dynamic light scattering with Malvern Nano ZS. The intensity of scattered light was detected at $90^{\circ}$.

\subsubsection{MTT assay}

Drug resistant prostate cancer cell DU145-TXR was used to determine the cell growth inhibition ability of drug-loaded micelles. Cells were cultured in DMEM media supplemented with $10 \%$ fetal bovine serum (FBS) and $1 \%$ antibiotic-antimycotic at $37{ }^{\circ} \mathrm{C}$ in humidified environment of $5 \% \mathrm{CO}_{2}$. Cells were seeded in $96-w e l l$ plates at a density of 5,000 cells per well. At the end of treatment, cell culture medium was replaced by $100 \mu \mathrm{L}$ medium with $0.5 \mathrm{mg} / \mathrm{mL}$ MTT (3-(4,5-dimethyl-thiazol-2-yl)-2, 5-diphenyl tetrazolium bromide and incubated for $1 \mathrm{~h}$ at $37^{\circ} \mathrm{C}$. Then medium was removed and $200 \mu \mathrm{L}$ of DMSO was added into each well to dissolve the formazan crystals. The absorbance was measured in a microplate reader at a wavelength of $560 \mathrm{~nm}$. Cell viability was expressed as the percentage of control group. Cell viability $(\%)=\left(\mathrm{A}_{\text {test }} / \mathrm{A}_{\text {control }}\right) \times 100 \%$.

\subsubsection{Calcein AM assay}

Cells were seeded into a black wall clear bottom 96-well plate at a density of 20,000 cells

per well one day before experiment. After treating cells with various concentration of test compounds in $50 \mu \mathrm{L}$ DPBS for $20 \mathrm{~min}$ at $37{ }^{\circ} \mathrm{C}, 50 \mu \mathrm{L}$ Calceim AM $(10 \mu \mathrm{M})$ in DPBS were added to each well and incubated at $37{ }^{\circ} \mathrm{C}$ for additional $20 \mathrm{~min}$. Fluorescence 
intensity in each well were determined using a SpectraMax M2/M2e spectrofluorometer (Sunnyvale, CA) at the excitation wavelength of $494 \mathrm{~nm}$ and emission wavelength of 517 nm.

\subsubsection{Propidium iodide staining and cell cycle analysis}

Cells were cultured in a 24 -well plate to $90 \%$ confluence and then treated for $24 \mathrm{~h}$. Cells were trypsinized and fixed in 70\% ice-cold ethanol. After washing with PBS, cell pellet was re-suspended in $5 \mu \mathrm{g} / \mathrm{mL}$ propidium iodide staining solution for $15 \mathrm{~min}$ at room temperature. Cell cycle distribution was measured by flow cytometry (Becton, Dickinson, NJ). Results from 10,000 fluorescent events were obtained for analysis.

\subsubsection{TUNEL assay}

TdT-mediated dUTP Nick End Labeling (TUNEL) assay was performed using DeadEnd Fluorometric TUNEL System (Promega, Madison, WI). Briefly, DU145-TXR cells were grown on Lab-tek chamber slide and treated with lapatinib, paclitaxel, and their combination for $24 \mathrm{~h}$. At the end of treatment, cells were fixed with $-20^{\circ} \mathrm{C}$ acetone for 10 min, washed with PBS, and equilibrated with buffer at RT for $10 \mathrm{~min}$. Then, it was incubated with staining agent composed of nucleotide and rTdT, in a humidified chamber at $37{ }^{\circ} \mathrm{C}$ for $1 \mathrm{~h}$ and protected from light. The reaction was terminated with $2 \mathrm{X} \mathrm{SSC}$ buffer and washed with PBS to remove unincorporated fluorescein-12-dUTP. The washed specimens were then counterstained with DAPI and visualized with Zeiss AxioVision fluorescent microscope.

\subsubsection{In vivo anticancer efficacy study}

All animal experiments were performed in accordance with $\mathrm{NIH}$ animal use guidelines and the protocol approved by the Animal Care and Use Committee at the University of Tennessee Health Science Center. Xenograft flank tumors were established in 8 week old male athymic nude mice (Charles River) by subcutaneously injection of five million DU145-TXR prostate cancer cells suspended in 1:1 media and matrigel. When tumors reached approximately $100 \mathrm{~mm}^{3}$, mice were randomized assigned to different treatments. Drugs were administrated through tail vain injection. Tumors were measured with a caliper prior to each injection, and their volumes were calculated using the formula: $\left(\right.$ width $^{2} \times$ length) $/ 2$. At the end of study, tumor tissue was excised from the mice and weighted. 


\subsection{Results and Discussion}

7.3.1. Effect of lapatinib and paclitaxel on MDR prostate cancer cells

We determined whether lapatinib could reverse MDR in prostate cancer cells. Paclitaxel resistant prostate cells DU145-TXR were treated with the combination of paclitaxel and lapatinib. As shown in Figure 7.2, the $\mathrm{IC}_{50}$ of paclitaxel was $2069 \pm 597 \mathrm{nM}$ in the absence of lapatinib, indicating DU145-TXR cells were resistant to paclitaxel. However, the $\mathrm{IC}_{50}$ of paclitaxel decreased to $79 \pm 29 \mathrm{nM}$, at lapatinib concentration of $1 \mu \mathrm{M}$, and to $15 \pm 8 \mathrm{nM}$ at lapatinib concentration of $5 \mu \mathrm{M}$. Fold of reversal was 26.2 and 138 at the lapatinib concentration of $1 \mu \mathrm{M}$ and $5 \mu \mathrm{M}$, respectively. The results showed that lapatinib can reverse the sensitivity of DU145-TXR to paclitaxel. This study proved the feasibility of using lapatinib and paclitaxel combination to overcome MDR in prostate cancers.

7.3.2. Preparation of paclitaxel and lapatinib loaded PEG-PCD lipopolymer micelle formulations

\subsubsection{Synthesis and characterization of PEG-PCD lipopolymer}

PEG-PCD lipopolymer were synthesized according our previous developed methods. The synthesized PEG-PCD lipopolymer were characterized with ${ }^{1} \mathrm{H}$ NMR, GPC. The Mn calculated based on 1H NMR is 11560 . We also used GPC to determine the apparent Mn and Mw of lipopolymer PEG-PCD, which was 10800 and 14986, respectively.

\subsubsection{Preparation and characterization of drug loaded lipopolymer formulations}

A film dispersion method was used to prepare lipopolymer micelles. The paclitaxel and lapatinib drug concentration in the formulations were determined with a HPLC method. Due to the different retention time for paclitaxel $(4.7 \mathrm{~min})$ and lapatinib $(7.1 \mathrm{~min})$, we can simultaneously determine the concentration of these two drugs (Figure 7.3A). We first tested the ability of lipopolymer PEG-PCD micelles to load a single drug of paclitaxel or lapatinib. As shown in Figure 7.3B and C, both paclitaxel and lapatinib can be effectively loaded into lipopolymer micelles. For both of these two drugs, the final drug concentrations in the formulation increase from around 0.5 to $2 \mathrm{mg} / \mathrm{mL}$ with increased theoretical loading (weight ratio of initial drug/polymer) from $5 \%$ to $20 \%$. The drug loading efficiency were around $100 \%$, indicating all the drugs can be effectively loaded into micelles at this range. Based on above findings, we developed several lipopolymer micelle formulations to simultaneously load paclitaxel and lapatinib. As shown in Figure 7.3D, we could simultaneously load both paclitaxel and lapatinib into lipopolymer micelles and reach final drug concentration to at least $1 \mathrm{mg}$ for each drug. The drug loading efficiencies were also around $100 \%$ for both drugs in all of these three formulations. We also determined the particle size of lipopolymer micelles with or without drug. The particle size was around $60 \mathrm{~nm}$ for all the tested formulations and the incorporation of drugs had almost no effect on particle size. 


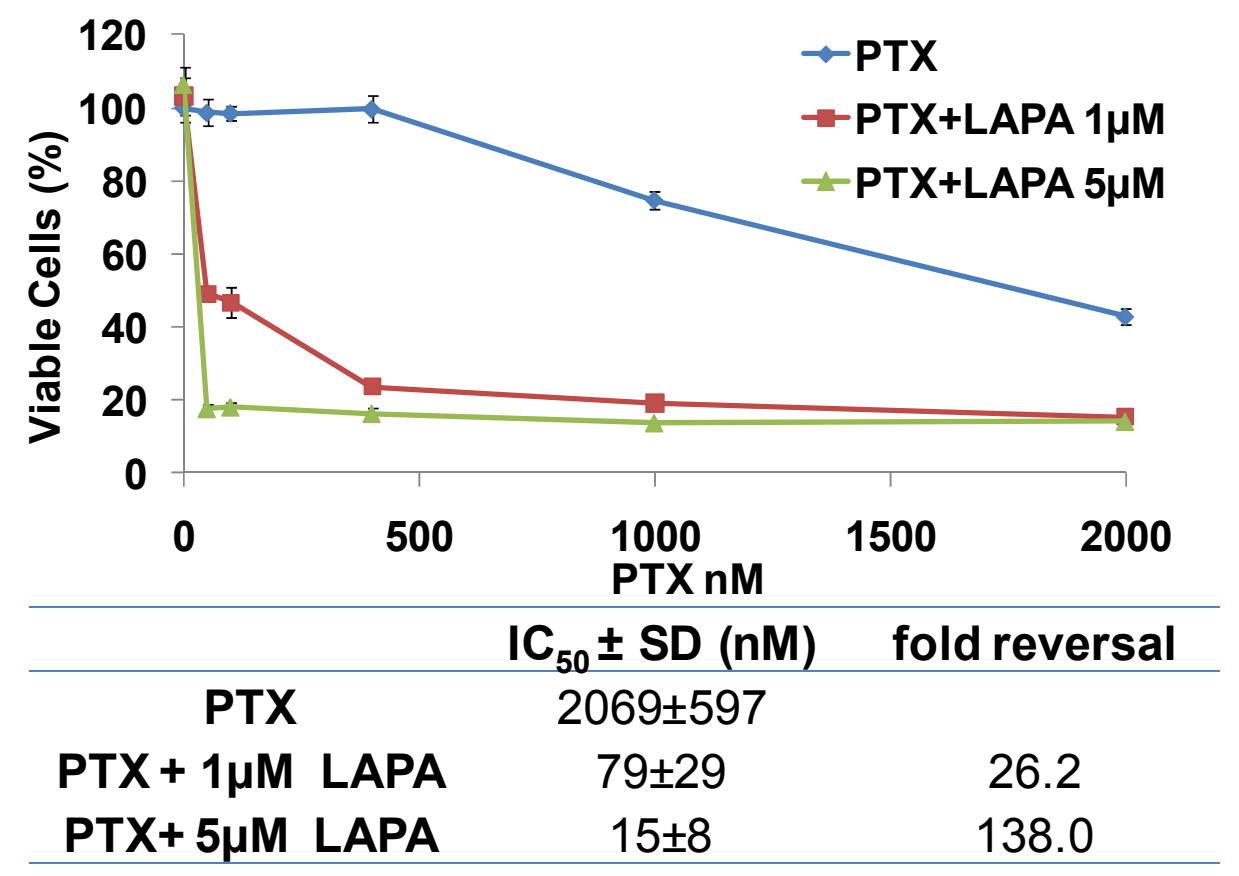

Figure 7.2 Effect of lapatinib on reversing MDR in DU145-TXR cells.

Percentage of viable cells was determined by MTT assay at $48 \mathrm{~h}$ post treatment. Data are the means $\pm \mathrm{SD}(\mathrm{n}=4)$. Fold of reversal $=\mathrm{IC}_{50 \text {, with lapabinib }} / \mathrm{IC}_{50, \text { no lapatinib. }}$ PTX, paclitaxel; LAPA, lapatinib. 

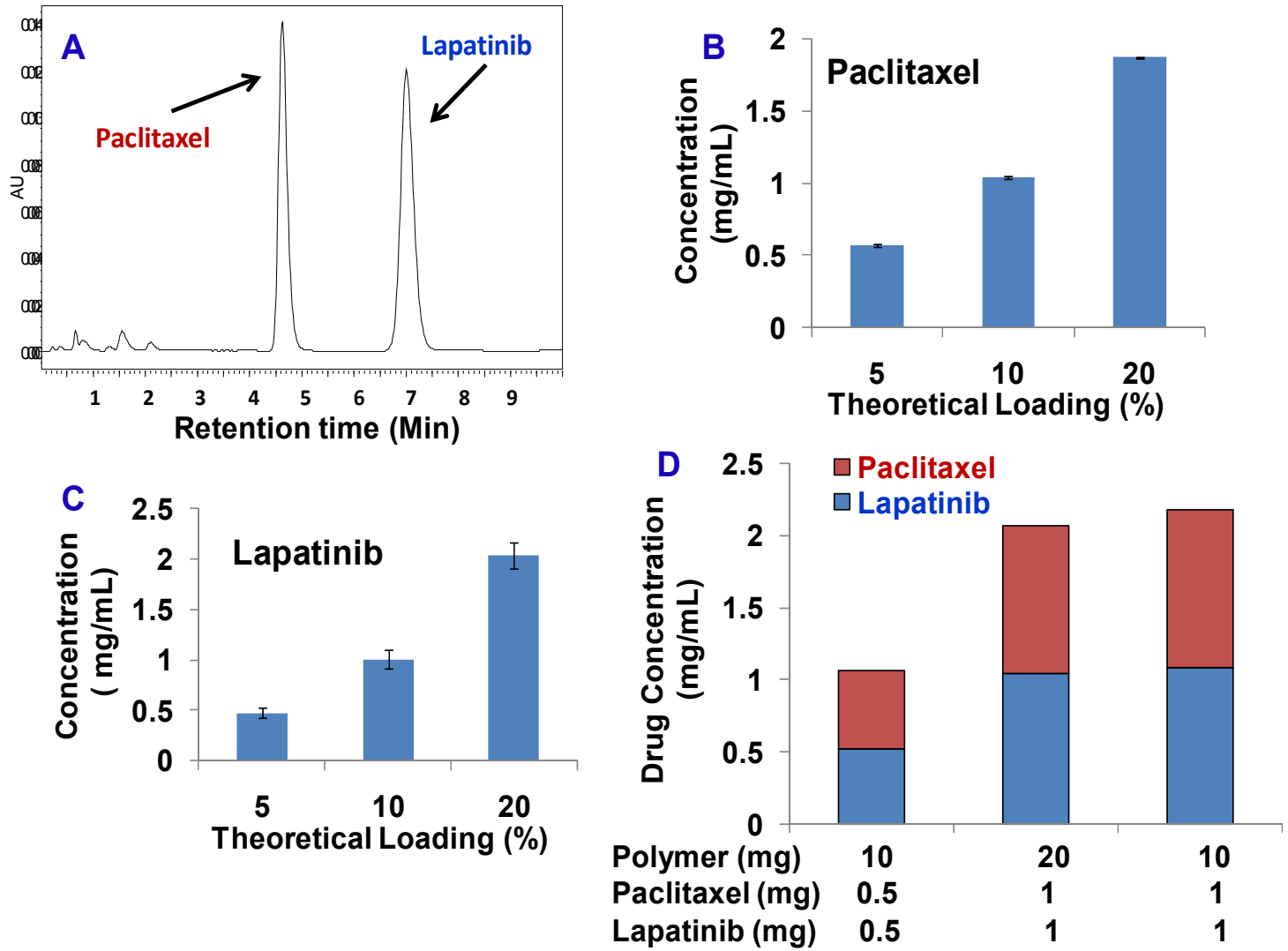

Figure 7.3 Determine drug concentrations in paclitaxel and/or lapatinib loaded lipopolymer micelles.

(A) HPLC chromatography of paclitaxel and lapatinib; (B) Effect of theoretical loading on paclitaxel solubility; (C) Effect of theoretical loading on lapatinib solubility; (D) drug concentration in paclitaxel and lapatinib co-loaded lipopolymer micelles. 
7.3.3. Inhibition of MDR prostate cancer cell proliferation by paclitaxel and lapatinib loaded lipopolymer micelles

The anticancer effect of paclitaxel and lapatinib loaded lipopolymer micelles were first tested in vitro with MDR prostate cancer cells DU145-TXR (Figure 7.4). No obvious toxicity was observed in cells treated with blank micelles, lapatinib $(2 \mu \mathrm{M})$, or paclitaxel $(0.5 \mu \mathrm{M})$ alone for $48 \mathrm{~h}$, while the combination of lapatinib $(2 \mu \mathrm{M})$ and paclitaxel $(0.5$ $\mu \mathrm{M}$ ) significantly reduced cell viability to around $23 \%$ of the control group. In addition, the combination of lapatinib $(1 \mu \mathrm{M})$ and paclitaxel $(0.25 \mu \mathrm{M})$ also showed significant inhibition of DU145-TXR cell proliferation. (Cell viability was around 35\% of the control group). This study further confirmed the lapatinib and paclitaxel loaded lipopolymer micelles formulation can overcome MDR in prostate cells and effectively inhibit the proliferation of resistant prostate cancer cells in vitro.

\subsubsection{Inhibition of P-gp activity in MDR cancer cells by lapatinib}

To understand the mechanism that lapatinib overcomes MDR in cancer cells, we performed a calcein AM assay. Calcein AM assay was used to determine the effect of lapatinib on P-gp activity [348]. The intracellular accumulation of calcein, which is a Pgp substrate with fluorescence, was observed with a fluorescence microscope and also quantitatively determined by a spectrofluorometer. As shown in Figure 7.5A, lapatinib treatment led to dose-dependent increase in intracellular calcein fluorescence. The fluorescence intensity increased with increased lapatinib concentration from 0 to $20 \mu \mathrm{M}$. We also quantitatively determined the fluorescent intensity with a spectrofluorometer (Figure 7.5B). In the absence of lapatinib the fluorescence intensity was very low (around $50 \mathrm{RFU}$ ), however, it increased to around $800 \mathrm{RFU}$ at a lapatinib concentration of $20 \mu \mathrm{M}$. In contrast, treatment with blank PEG-PCD lipopolymer micelles showed neglectable effect on intracellular accumulation of calcein fluorescence. Since calcein AM is a P-gp substrate, the observed results indicate that lapatinib works as an inhibitor of P-gp, reduces the P-gp substances efflux, and thus increases the intracellular accumulation of P-gp substances (Such as paclitaxel).

7.3.5. Effect of paclitaxel and lapatinib loaded micelles on cell cycle and apoptosis of MDR prostate cancer cells

We also determined the effect of lapatinib and paclitaxel loaded lipopolymer micelles on DU145-TXR cells by analyzing cell cycle distribution and cell apoptosis with a flow cytometry. Cells were treated with different formulations for $24 \mathrm{~h}$ before analysis. As shown in Figure 7.6, the treatment of lapatinib $(2 \mu \mathrm{M})$ or paclitaxel $(0.5 \mu \mathrm{M})$ alone had no effects on the cycle distribution, in contrast, their combination showed significant effects on cycle distribution and cell apoptosis. The treatment of lapatinib $(2 \mu \mathrm{M})+$ paclitaxel $(0.5 \mu \mathrm{M})$ caused cells in $\mathrm{G} 0 / \mathrm{G} 1$ phase decreased from $29.0 \pm 2.1 \%$ in control group to group to $4.7 \pm 0.2 \%$ in the combination group. Meanwhile, cells in $\mathrm{G} 2 / \mathrm{M}$ phase 


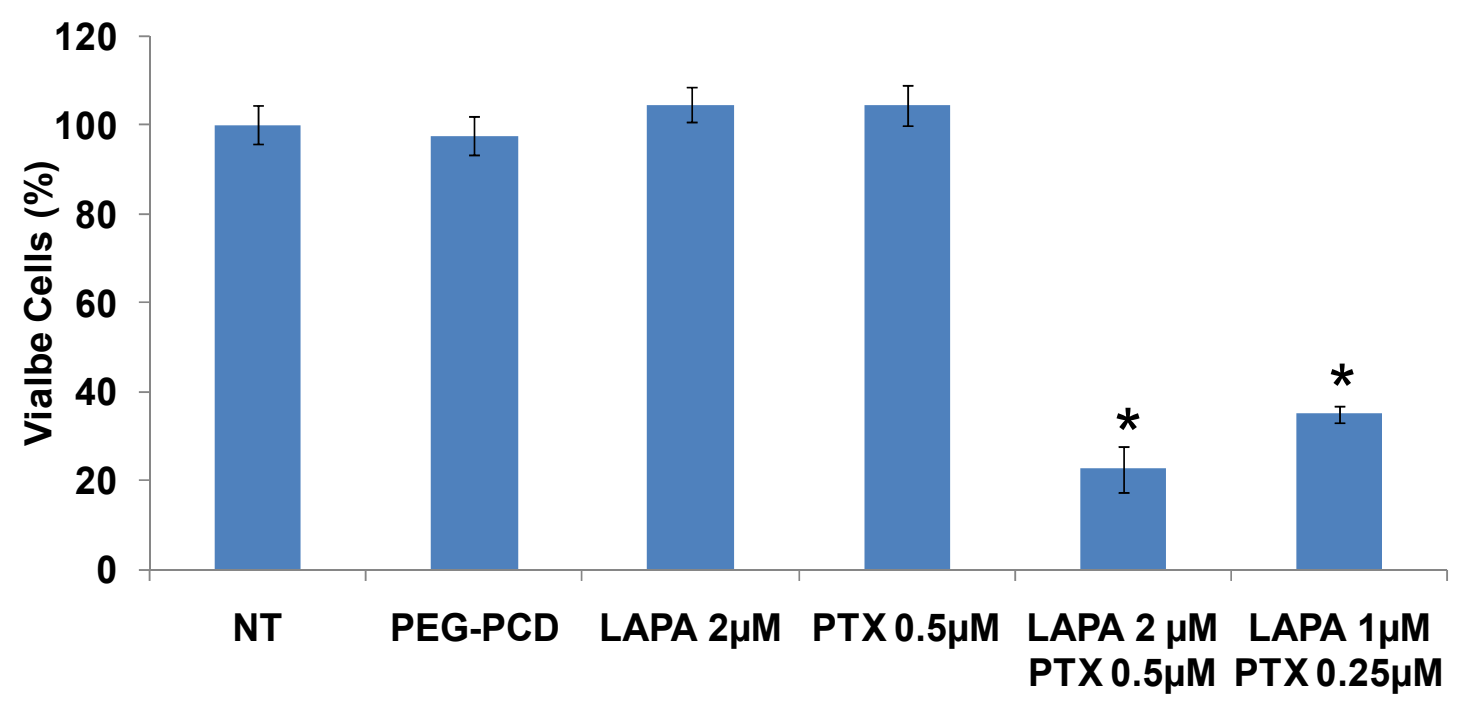

Figure 7.4 Anticancer effect of paclitaxel and lapatinib combination on MDR DU145-TXR cells.

DU145-TXR cells were treated for $48 \mathrm{~h}$, and then cell viability was determined by MTT assay and expressed as the percentage of control. *, p $<0.01$, compare with non-treated control (NT). 
A
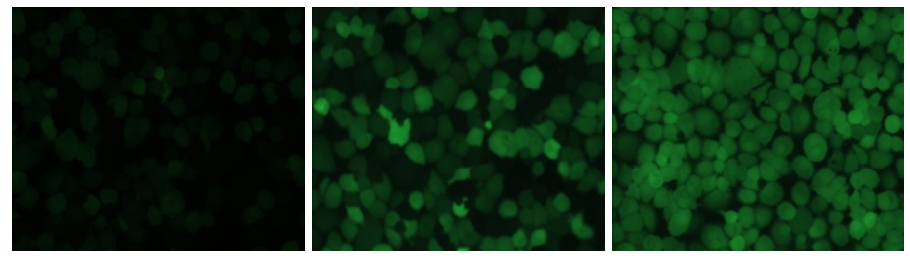

5

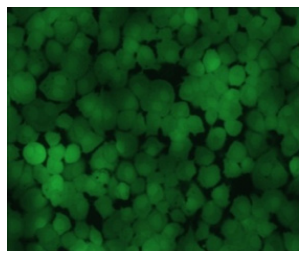

0

1

\section{0}

B

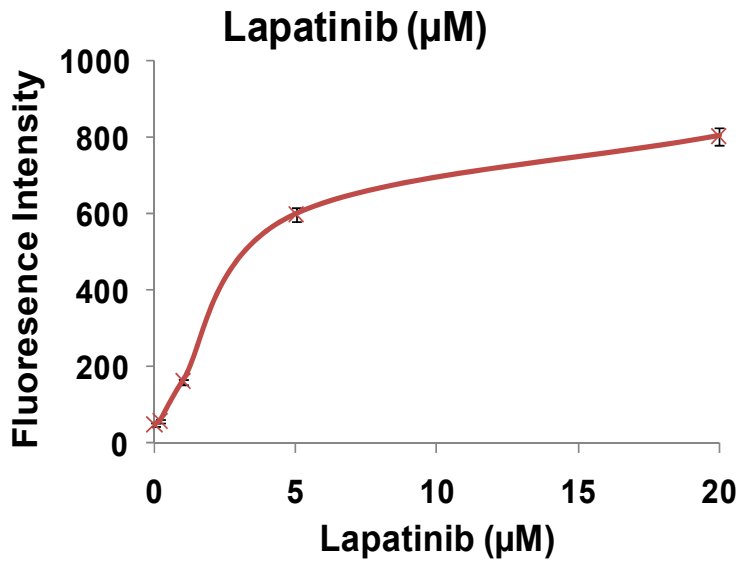

Figure 7.5 Inhibition of P-gp activity by lapatinib.

The effects of lapatinib on intracelluar accumulation of calcein (P-gp substrate) in DU145-TXR cells were determined with (A) Fluorescence microscope, and (B). spectrofluorometer. 


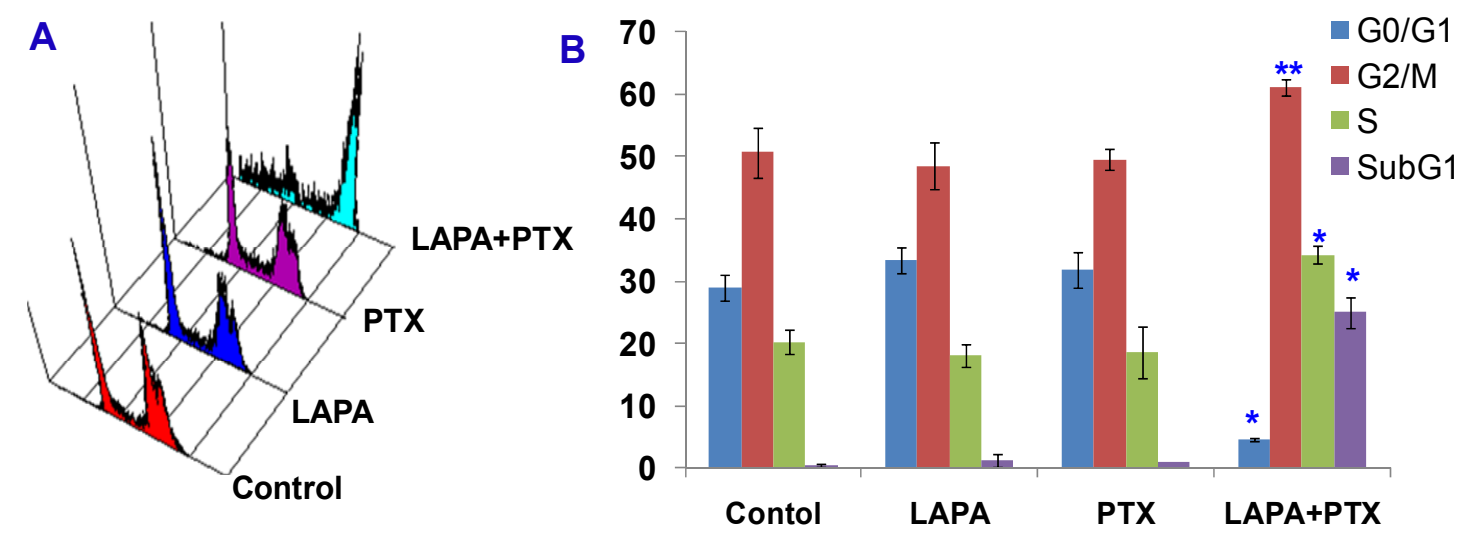

Figure 7.6 Effect of paclitaxel and lapatinib combination on cell cycle and apoptosis of MDR prostate cancers.

DU145-TXR cells were treated with PEG-PCD micelles formulated Lapatinib $(2 \mu \mathrm{M})$, Paclitaxel $(0.5 \mu \mathrm{M})$, and Lapatinib $(2 \mu \mathrm{M})+$ Paclitaxel $(0.5 \mu \mathrm{M})$. After $24 \mathrm{~h}$ treatment, cell cycles were analyzed by flow cytometry after PI staining. (A) Cell cycle distribution; (B) Quantitative analysis. *, p $<0.01,{ }^{*}, \mathrm{p}<0.05$, compare with control. 
were increased from $50.7 \pm 4.0 \%$ in control group to $61.1 \pm 1.2 \%$ in the combination group. In addition, the apoptotic subG1 phase cells increased from $0.4 \pm 0.2 \%$ in the control group to $25.1 \pm 2.5 \%$ in the combination group. These results indicated that the cells treated with above combination therapy were failed to undergo mitosis. This is consistent with the anticancer mechanism of paclitaxel, which is a microtubule inhibitor and can cause the arrest of the cell cycle at mitotic phase. The increased cell population in subG1 phase indicated that apoptotic cells were increased after treating with combination therapy [332332]. We also used TUNEL assay to further confirm that the combination of paclitaxel and lapatinib induced apoptosis in DU145-TXR cells. TUNEL is a method for detecting DNA fragmentation by labeling the terminal end of nucleic acids. As shown in Figure 7.7 , neither paclitaxel $(0.5 \mu \mathrm{M})$ nor lapatinib $(2 \mu \mathrm{M})$ alone caused apoptotic cell death in DU145-TXR cells, while the combination of paclitaxel $(0.5$ $\mu \mathrm{M})$ and lapatinib $(2 \mu \mathrm{M})$ induced significant cell apoptosis.

\subsubsection{Lapatinib and paclitaxel loaded lipopolymer micelles overcome MDR in vivo}

Encouraged by the in vitro anticancer effect of lapatinib and paclitaxel loaded lipopolymer micelles in MDR prostate cancer cells. We decided to test their ability to overcome MDR in vivo using athymic nude mice xenograft model established with DU145-TXT MDR prostate cancer cells. When tumors reached approximately $100 \mathrm{~mm}^{3}$, mice were randomized assigned to two groups of four mice. Mice were treated with tail vain injection of lipopolymer micelle formulations at a dose of paclitaxel $(10 \mathrm{mg} / \mathrm{kg})$ in monotherapy group or paclitaxel $(5 \mathrm{mg} / \mathrm{kg})+$ lapatinib $(5 \mathrm{mg} / \mathrm{kg})$ in combination therapy group, respectively. In contrast to paclitaxel monothearpy, the lapatinib and paclitaxel combination therapy significantly inhibited in vivo tumor growth (Figure 7.8A). We also determined the weight of tumor at the end of the study, which also showed that the weight of tumors in combination group were significantly less than those in the paclitaxel monotherapy group $(\mathrm{P}<0.05$, Figure $7.8 \mathrm{~B})$. In addition, we did not observe any mortality and significant decrease body weight in mice after drug administration, indicating the neglectable toxicity of lipopolymer micelle formulations used in this study (Figure 7.8C).

\subsection{Summary and Conclusion}

Paclitaxel and lapatinib loaded lipopolymer micelle formulations were developed, which showed high drug loading efficiency. These lipopolymer micelle formulations have a particle size of around $60 \mathrm{~nm}$ and narrow size distribution. In vitro cell-based studies showed that paclitaxel and lapatinib loaded lipopolymer micelles can overcome MDR in prostate cancers. The paclitaxel $(0.5 \mu \mathrm{M})$ and lapatinib $(2 \mu \mathrm{M})$ combination effectively inhibited DU145-TXR cell proliferation in vitro, induced cell cycle perturbation and cell apoptosis. In contrast, monotherapy with paclitaxel $(0.5 \mu \mathrm{M})$ or lapatinib $(2 \mu \mathrm{M})$ alone showed almost no effect on DU145-TXR cells. Effect of combination therapy was further investigated in vivo with athymic nude mice xenograft tumor model established with MDR prostate cancer cells. Similar to in vitro study, paclitaxel (5 mg/kg) + lapatinib (5 $\mathrm{mg} / \mathrm{Kg}$ ) combination therapy significantly inhibited tumor growth in vivo compared with 


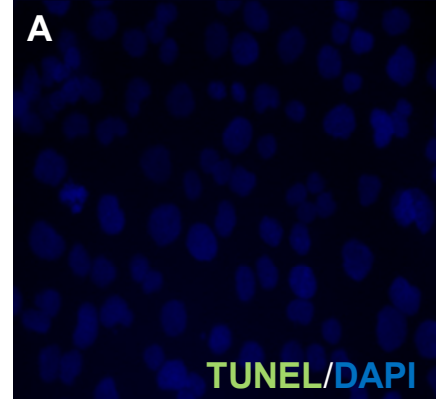

Paclitaxel $0.5 \mu \mathrm{M}$

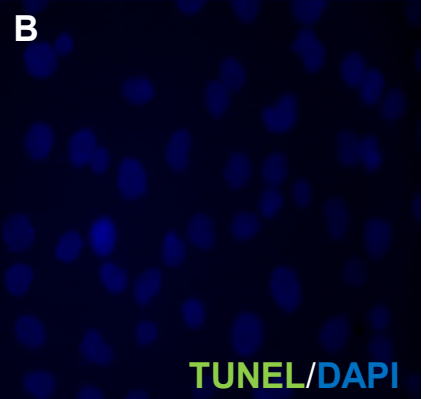

Lapatinib $2 \mu \mathrm{M}$

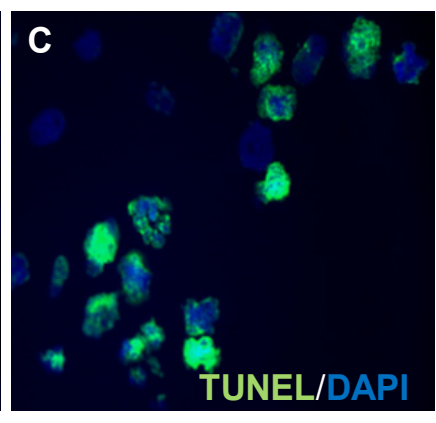

Paclitaxel $0.5 \mu \mathrm{M}$ +Lapatinib $2 \mu \mathrm{M}$

Figure 7.7 Detection of paclitaxel and lapatinib combination induced cell apoptosis with TUNEL staining.

Paclitaxel and Lapatinib combination induced cell apoptosis in DU145-TXR Cells. DU145 Cells were treated with (A) paclitaxel $0.5 \mu \mathrm{M}$, (B) Lapatinib $2 \mu \mathrm{M}$, and (C) paclitaxel $0.5 \mu \mathrm{M}+$ lapatinib $2 \mu \mathrm{M}$. After $24 \mathrm{~h}$ treatment, cells were subject to TUNEL Staining and counterstained with DAPI. Images are overlay of green fluorescence from the TUNEL stain with blue fluorescence from DAPI. 
A
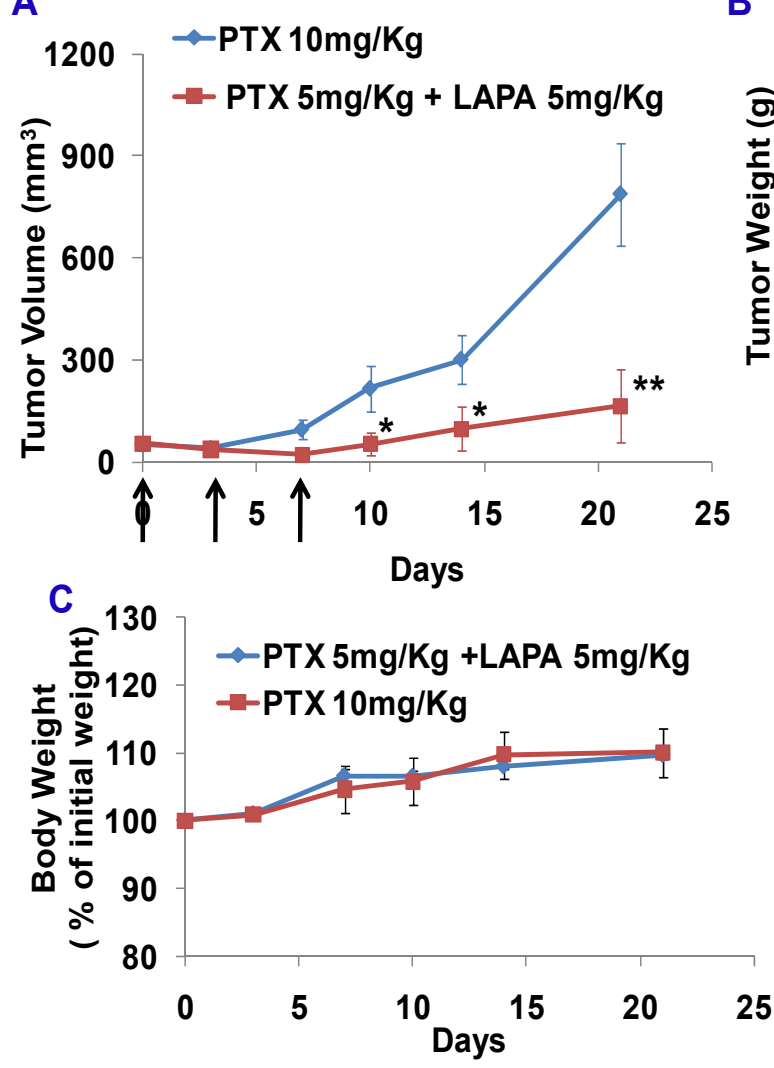

B

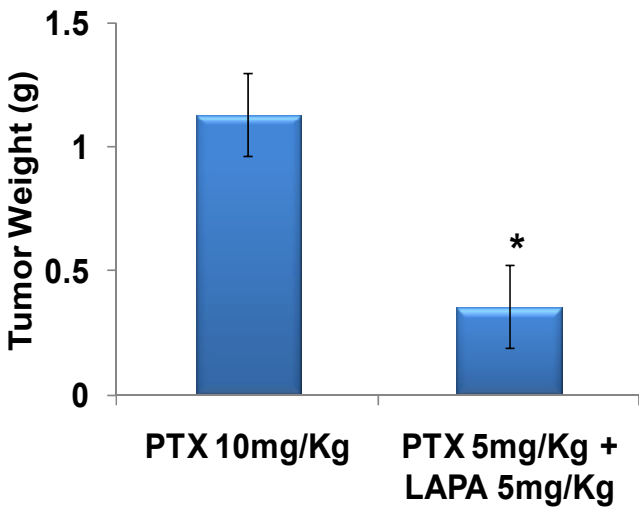

Figure 7.8 In vivo anticancer effect of paclitaxel and lapatinib loaded lipopolymer micelles on xenograft MDR prostate tumors.

MDR xenograft athymic tumor models were established with DU145-TXR prostate cancer cells. When the tumor size reaches $100 \mathrm{~mm}^{3}$, mice were given intravenously injection of lipopolymer formulations on day 0,3 , and 4 , respectively. The dose was 10 $\mathrm{mg} / \mathrm{Kg}$ paclitaxel for paclitaxel monotherarpy group, $5 \mathrm{mg} / \mathrm{kg}$ each of lapatinib and paclitaxel in combination therapy group. Tumor growth was measured with a caliper and tumor size was calculated using the formula: (width ${ }^{2} \times$ length) $/ 2(\mathrm{~A})$. At the end of study, tumor tissue was excised from the mice and weighted (B). Meanwhile, the body weight of mice were also determined (C). Points are mean tumor size $(\mathrm{n}=4)$; bars, SE. *, $\mathrm{p}<$ $0.01, * *, \mathrm{p}<0.05$. 
high dose paclitaxel $(10 \mathrm{mg} / \mathrm{kg})$ monothearpy. These studies indicate that the paclitaxel and lapatinib loaded PEG-PCD lipopolymer micelle formulations could be used to treat MDR prostate cancers. 


\section{LIST OF REFERENCES}

1. Y. Matsumura, K. Kataoka, Preclinical and clinical studies of anticancer agentincorporating polymer micelles, Cancer Sci, 100 (2009) 572-579.

2. C. Oerlemans, W. Bult, M. Bos, G. Storm, J.F. Nijsen, W.E. Hennink, Polymeric micelles in anticancer therapy: targeting, imaging and triggered release, Pharm Res, 27 (2010) 2569-2589.

3. P.W. Williams, Notes on diabetes treated with extract and by grafts of sheep's pancreas, Br Med J, 2 (1894) 1303-1304.

4. W.D. Kelly, R.C. Lillehei, F.K. Merkel, Y. Idezuki, F.C. Goetz, Allotransplantation of the pancreas and duodenum along with the kidney in diabetic nephropathy, Surgery, 61 (1967) 827-837.

5. M. Vardanyan, E. Parkin, C. Gruessner, H.L. Rodriguez Rilo, Pancreas vs. islet transplantation: a call on the future, Curr Opin Organ Transplant, 15 (2010) 124130.

6. C. Troppmann, Complications after pancreas transplantation, Curr Opin Organ Transplant, 15 (2010) 112-118.

7. A.M. Shapiro, J.R. Lakey, E.A. Ryan, G.S. Korbutt, E. Toth, G.L. Warnock, N.M. Kneteman, R.V. Rajotte, Islet transplantation in seven patients with type 1 diabetes mellitus using a glucocorticoid-free immunosuppressive regimen, N Engl J Med, 343 (2000) 230-238.

8. R. Alejandro, F.B. Barton, B.J. Hering, S. Wease, 2008 Update from the collaborative islet transplant registry, Transplantation, 86 (2008) 1783-1788.

9. S. Merani, A.M. Shapiro, Current status of pancreatic islet transplantation, Clin Sci (Lond), 110 (2006) 611-625.

10. E.A. Ryan, B.W. Paty, P.A. Senior, D. Bigam, E. Alfadhli, N.M. Kneteman, J.R. Lakey, A.M. Shapiro, Five-year follow-up after clinical islet transplantation, Diabetes, 54 (2005) 2060-2069.

11. D.M. Harlan, N.S. Kenyon, O. Korsgren, B.O. Roep, Current advances and travails in islet transplantation, Diabetes, 58 (2009) 2175-2184.

12. A.S. Narang, R.I. Mahato, Biological and biomaterial approaches for improved islet transplantation, Pharmacol Rev, 58 (2006) 194-243. 
13. A.M. Shapiro, C. Ricordi, B.J. Hering, H. Auchincloss, R. Lindblad, R.P. Robertson, A. Secchi, M.D. Brendel, T. Berney, D.C. Brennan, E. Cagliero, R. Alejandro, E.A. Ryan, B. DiMercurio, P. Morel, K.S. Polonsky, J.A. Reems, R.G. Bretzel, F. Bertuzzi, T. Froud, R. Kandaswamy, D.E. Sutherland, G. Eisenbarth, M. Segal, J. Preiksaitis, G.S. Korbutt, F.B. Barton, L. Viviano, V. SeyfertMargolis, J. Bluestone, J.R. Lakey, International trial of the Edmonton protocol for islet transplantation, N Engl J Med, 355 (2006) 1318-1330.

14. H. Miszta-Lane, M. Mirbolooki, A.M. James Shapiro, J.R. Lakey, Stem cell sources for clinical islet transplantation in type 1 diabetes: embryonic and adult stem cells, Med Hypotheses, 67 (2006) 909-913.

15. K. Cheng, D. Fraga, C. Zhang, M. Kotb, A.O. Gaber, R.V. Guntaka, R.I. Mahato, Adenovirus-based vascular endothelial growth factor gene delivery to human pancreatic islets, Gene Ther, 11 (2004) 1105-1116.

16. R.I. Mahato, J. Henry, A.S. Narang, O. Sabek, D. Fraga, M. Kotb, A.O. Gaber, Cationic lipid and polymer-based gene delivery to human pancreatic islets, Mol Ther, 7 (2003) 89-100.

17. A.S. Narang, K. Cheng, J. Henry, C. Zhang, O. Sabek, D. Fraga, M. Kotb, A.O. Gaber, R.I. Mahato, Vascular endothelial growth factor gene delivery for revascularization in transplanted human islets, Pharm Res, 21 (2004) 15-25.

18. R. Panakanti, R.I. Mahato, Bipartite vector encoding hVEGF and hIL-1Ra for ex vivo transduction into human islets, Mol Pharm, 6 (2009) 274-284.

19. R. Panakanti, R.I. Mahato, Bipartite adenoviral vector encoding hHGF and hIL1Ra for improved human islet transplantation, Pharm Res, 26 (2009) 587-596.

20. R.I. Mahato, Gene expression and silencing for improved islet transplantation, $\mathrm{J}$ Control Release, 140 (2009) 262-267.

21. A. Fire, S. Xu, M.K. Montgomery, S.A. Kostas, S.E. Driver, C.C. Mello, Potent and specific genetic interference by double-stranded RNA in Caenorhabditis elegans, Nature, 391 (1998) 806-811.

22. M.K. Montgomery, S. Xu, A. Fire, RNA as a target of double-stranded RNAmediated genetic interference in Caenorhabditis elegans, Proc Natl Acad Sci U S A, 95 (1998) 15502-15507.

23. T. Nguyen, E.M. Menocal, J. Harborth, J.H. Fruehauf, RNAi therapeutics: an update on delivery, Curr Opin Mol Ther, 10 (2008) 158-167. 
24. A. de Fougerolles, H.P. Vornlocher, J. Maraganore, J. Lieberman, Interfering with disease: a progress report on siRNA-based therapeutics, Nat Rev Drug Discov, 6 (2007) 443-453.

25. D. De Paula, M.V. Bentley, R.I. Mahato, Hydrophobization and bioconjugation for enhanced siRNA delivery and targeting, Rna, 13 (2007) 431-456.

26. Y. Chen, G. Cheng, R.I. Mahato, RNAi for treating hepatitis B viral infection, Pharm Res, 25 (2008) 72-86.

27. L.J. Scherer, J.J. Rossi, Approaches for the sequence-specific knockdown of mRNA, Nat Biotechnol, 21 (2003) 1457-1465.

28. L. Scherer, J.J. Rossi, RNAi applications in mammalian cells, Biotechniques, 36 (2004) 557-561.

29. J.R. Bertrand, M. Pottier, A. Vekris, P. Opolon, A. Maksimenko, C. Malvy, Comparison of antisense oligonucleotides and siRNAs in cell culture and in vivo, Biochem Biophys Res Commun, 296 (2002) 1000-1004.

30. M. Miyagishi, M. Hayashi, K. Taira, Comparison of the suppressive effects of antisense oligonucleotides and siRNAs directed against the same targets in mammalian cells, Antisense Nucleic Acid Drug Dev, 13 (2003) 1-7.

31. D.J. van der Windt, R. Bottino, A. Casu, N. Campanile, D.K. Cooper, Rapid loss of intraportally transplanted islets: an overview of pathophysiology and preventive strategies, Xenotransplantation, 14 (2007) 288-297.

32. W. Bennet, C.G. Groth, R. Larsson, B. Nilsson, O. Korsgren, Isolated human islets trigger an instant blood mediated inflammatory reaction: implications for intraportal islet transplantation as a treatment for patients with type 1 diabetes, Ups J Med Sci, 105 (2000) 125-133.

33. O. Korsgren, B. Nilsson, C. Berne, M. Felldin, A. Foss, R. Kallen, T. Lundgren, K. Salmela, A. Tibell, G. Tufveson, Current status of clinical islet transplantation, Transplantation, 79 (2005) 1289-1293.

34. L. Moberg, H. Johansson, A. Lukinius, C. Berne, A. Foss, R. Kallen, O. Ostraat, K. Salmela, A. Tibell, G. Tufveson, G. Elgue, K. Nilsson Ekdahl, O. Korsgren, B. Nilsson, Production of tissue factor by pancreatic islet cells as a trigger of detrimental thrombotic reactions in clinical islet transplantation, Lancet, 360 (2002) 2039-2045. 
35. H. Johansson, A. Lukinius, L. Moberg, T. Lundgren, C. Berne, A. Foss, M. Felldin, R. Kallen, K. Salmela, A. Tibell, G. Tufveson, K.N. Ekdahl, G. Elgue, O. Korsgren, B. Nilsson, Tissue factor produced by the endocrine cells of the islets of langerhans is associated with a negative outcome of clinical islet transplantation, Diabetes, 54 (2005) 1755-1762.

36. W. Bennet, B. Sundberg, C.G. Groth, M.D. Brendel, D. Brandhorst, H. Brandhorst, R.G. Bretzel, G. Elgue, R. Larsson, B. Nilsson, O. Korsgren, Incompatibility between human blood and isolated islets of langerhans: a finding with implications for clinical intraportal islet transplantation, Diabetes, 48 (1999) 1907-1914.

37. W. Bennet, B. Sundberg, T. Lundgren, A. Tibell, C.G. Groth, A. Richards, D.J. White, G. Elgue, R. Larsson, B. Nilsson, O. Korsgren, Damage to porcine islets of langerhans after exposure to human blood in vitro, or after intraportal transplantation to cynomologus monkeys: protective effects of sCR1 and heparin, Transplantation, 69 (2000) 711-719.

38. L. Moberg, O. Korsgren, B. Nilsson, Neutrophilic granulocytes are the predominant cell type infiltrating pancreatic islets in contact with ABOcompatible blood, Clin Exp Immunol, 142 (2005) 125-131.

39. J.L. Contreras, C. Eckstein, C.A. Smyth, G. Bilbao, M. Vilatoba, S.E. Ringland, C. Young, J.A. Thompson, J.A. Fernandez, J.H. Griffin, D.E. Eckhoff, Activated protein $\mathrm{C}$ preserves functional islet mass after intraportal transplantation: a novel link between endothelial cell activation, thrombosis, inflammation, and islet cell death, Diabetes, 53 (2004) 2804-2814.

40. F.M. Szaba, S.T. Smiley, Roles for thrombin and fibrin(ogen) in cytokine/chemokine production and macrophage adhesion in vivo, Blood, 99 (2002) 1053-1059.

41. N. Kirchhof, S. Shibata, M. Wijkstrom, D.M. Kulick, C.T. Salerno, S.M. Clemmings, Y. Heremans, U. Galili, D.E. Sutherland, A.P. Dalmasso, B.J. Hering, Reversal of diabetes in non-immunosuppressed rhesus macaques by intraportal porcine islet xenografts precedes acute cellular rejection, Xenotransplantation, 11 (2004) 396-407.

42. C. Gysemans, H. Callewaert, L. Overbergh, C. Mathieu, Cytokine signalling in the beta-cell: a dual role for IFNgamma, Biochem Soc Trans, 36 (2008) 328-333.

43. C.F. Nathan, Secretory products of macrophages, J Clin Invest, 79 (1987) 319326. 
44. K. Bendtzen, T. Mandrup-Poulsen, J. Nerup, J.H. Nielsen, C.A. Dinarello, M. Svenson, Cytotoxicity of human pI 7 interleukin-1 for pancreatic islets of langerhans, Science, 232 (1986) 1545-1547.

45. N.R. Barshes, S. Wyllie, J.A. Goss, Inflammation-mediated dysfunction and apoptosis in pancreatic islet transplantation: implications for intrahepatic grafts, $\mathrm{J}$ Leukoc Biol, 77 (2005) 587-597.

46. M. Arnush, M.R. Heitmeier, A.L. Scarim, M.H. Marino, P.T. Manning, J.A. Corbett, IL-1 produced and released endogenously within human islets inhibits beta cell function, J Clin Invest, 102 (1998) 516-526.

47. R. Bottino, L.A. Fernandez, C. Ricordi, R. Lehmann, M.F. Tsan, R. Oliver, L. Inverardi, Transplantation of allogeneic islets of Langerhans in the rat liver: effects of macrophage depletion on graft survival and microenvironment activation, Diabetes, 47 (1998) 316-323.

48. M. Montolio, M. Biarnes, N. Tellez, J. Escoriza, J. Soler, E. Montanya, Interleukin-1beta and inducible form of nitric oxide synthase expression in early syngeneic islet transplantation, J Endocrinol, 192 (2007) 169-177.

49. C.A. Gysemans, M. Waer, D. Valckx, J.M. Laureys, D. Mihkalsky, R. Bouillon, C. Mathieu, Early graft failure of xenogeneic islets in NOD mice is accompanied by high levels of interleukin-1 and low levels of transforming growth factor-beta mRNA in the grafts, Diabetes, 49 (2000) 1992-1997.

50. T. Ozasa, M.R. Newton, M.J. Dallman, S. Shimizu, D.W. Gray, P.J. Morris, Cytokine gene expression in pancreatic islet grafts in the rat, Transplantation, 64 (1997) 1152-1159.

51. H.E. Thomas, M.D. McKenzie, E. Angstetra, P.D. Campbell, T.W. Kay, Beta cell apoptosis in diabetes, Apoptosis, 14 (2009) 1389-1404.

52. D.L. Eizirik, T. Mandrup-Poulsen, A choice of death: the signal transduction of immune-mediated beta-cell apoptosis, Diabetologia, 44 (2001) 2115-2133.

53. G. Tau, P. Rothman, Biologic functions of the IFN-gamma receptors, Allergy, 54 (1999) 1233-1251.

54. A. Stephanou, B.K. Brar, T.M. Scarabelli, A.K. Jonassen, D.M. Yellon, M.S. Marber, R.A. Knight, D.S. Latchman, Ischemia-induced STAT-1 expression and activation play a critical role in cardiomyocyte apoptosis, J Biol Chem, 275 (2000) 10002-10008.

55. G. Chen, D.V. Goeddel, TNF-R1 signaling: a beautiful pathway, Science, 296 (2002) 1634-1635. 
56. H. Wajant, K. Pfizenmaier, P. Scheurich, Tumor necrosis factor signaling, Cell Death Differ, 10 (2003) 45-65.

57. P.C. Rath, B.B. Aggarwal, TNF-induced signaling in apoptosis, J Clin Immunol, 19 (1999) 350-364.

58. J. Saklatvala, J. Dean, A. Finch, Protein kinase cascades in intracellular signalling by interleukin-I and tumour necrosis factor, Biochem Soc Symp, 64 (1999) 63-77.

59. B. Kutlu, A.K. Cardozo, M.I. Darville, M. Kruhoffer, N. Magnusson, T. Orntoft, D.L. Eizirik, Discovery of gene networks regulating cytokine-induced dysfunction and apoptosis in insulin-producing INS-1 cells, Diabetes, 52 (2003) 2701-2719.

60. D.L. Eizirik, B. Kutlu, J. Rasschaert, M. Darville, A.K. Cardozo, Use of microarray analysis to unveil transcription factor and gene networks contributing to beta-cell dysfunction and apoptosis, Ann N Y Acad Sci, 1005 (2003) 55-74.

61. A.K. Cardozo, H. Heimberg, Y. Heremans, R. Leeman, B. Kutlu, M. Kruhoffer, T. Orntoft, D.L. Eizirik, A comprehensive analysis of cytokine-induced and nuclear factor-kappa B-dependent genes in primary rat pancreatic beta-cells, $\mathrm{J}$ Biol Chem, 276 (2001) 48879-48886.

62. F. Ortis, A.K. Cardozo, D. Crispim, J. Storling, T. Mandrup-Poulsen, D.L. Eizirik, Cytokine-induced proapoptotic gene expression in insulin-producing cells is related to rapid, sustained, and nonoscillatory nuclear factor-kappaB activation, Mol Endocrinol, 20 (2006) 1867-1879.

63. J.A. Corbett, M.A. Sweetland, J.L. Wang, J.R. Lancaster, Jr., M.L. McDaniel, Nitric oxide mediates cytokine-induced inhibition of insulin secretion by human islets of langerhans, Proc Natl Acad Sci U S A, 90 (1993) 1731-1735.

64. J.A. Emamaullee, A.M. Shapiro, Interventional strategies to prevent beta-cell apoptosis in islet transplantation, Diabetes, 55 (2006) 1907-1914.

65. M.O. Hengartner, The biochemistry of apoptosis, Nature, 407 (2000) 770-776.

66. D.R. Green, Apoptotic pathways: paper wraps stone blunts scissors, Cell, 102 (2000) 1-4.

67. L. Jansson, Dissociation between pancreatic islet blood flow and insulin release in the rat, Acta Physiol Scand, 124 (1985) 223-228.

68. S. Sezai, S. Sakurabayashi, Y. Yamamoto, T. Morita, M. Hirano, H. Oka, Hepatic arterial and portal venous oxygen content and extraction in liver cirrhosis, Liver, 13 (1993) 31-35. 
69. A. Pileggi, C. Ricordi, M. Alessiani, L. Inverardi, Factors influencing islet of langerhans graft function and monitoring, Clin Chim Acta, 310 (2001) 3-16.

70. M.D. Menger, J. Yamauchi, B. Vollmar, Revascularization and microcirculation of freely grafted islets of langerhans, World J Surg, 25 (2001) 509-515.

71. X. Li, H. Chen, P.N. Epstein, Metallothionein protects islets from hypoxia and extends islet graft survival by scavenging most kinds of reactive oxygen species, $\mathrm{J}$ Biol Chem, 279 (2004) 765-771.

72. R.K. Sibley, D.E. Sutherland, F. Goetz, A.F. Michael, Recurrent diabetes mellitus in the pancreas iso- and allograft. A light and electron microscopic and immunohistochemical analysis of four cases, Lab Invest, 53 (1985) 132-144.

73. P. Santamaria, R.E. Nakhleh, D.E. Sutherland, J.J. Barbosa, Characterization of T lymphocytes infiltrating human pancreas allograft affected by isletitis and recurrent diabetes, Diabetes, 41 (1992) 53-61.

74. C. Mora, F.S. Wong, C.H. Chang, R.A. Flavell, Pancreatic infiltration but not diabetes occurs in the relative absence of MHC class II-restricted CD4 T cells: studies using NOD/CIITA-deficient mice, J Immunol, 162 (1999) 4576-4588.

75. J.A. Trapani, M.J. Smyth, Functional significance of the perforin/granzyme cell death pathway, Nat Rev Immunol, 2 (2002) 735-747.

76. V.R. Sutton, J.E. Davis, M. Cancilla, R.W. Johnstone, A.A. Ruefli, K. Sedelies, K.A. Browne, J.A. Trapani, Initiation of apoptosis by granzyme B requires direct cleavage of bid, but not direct granzyme B-mediated caspase activation, J Exp Med, 192 (2000) 1403-1414.

77. E. Estella, M.D. McKenzie, T. Catterall, V.R. Sutton, P.I. Bird, J.A. Trapani, T.W. Kay, H.E. Thomas, Granzyme B-mediated death of pancreatic beta-cells requires the proapoptotic BH3-only molecule bid, Diabetes, 55 (2006) 2212-2219.

78. M. Moriwaki, N. Itoh, J. Miyagawa, K. Yamamoto, A. Imagawa, K. Yamagata, H. Iwahashi, H. Nakajima, M. Namba, S. Nagata, T. Hanafusa, Y. Matsuzawa, Fas and Fas ligand expression in inflamed islets in pancreas sections of patients with recent-onset type I diabetes mellitus, Diabetologia, 42 (1999) 1332-1340.

79. A.C. Loweth, G.T. Williams, R.F. James, J.H. Scarpello, N.G. Morgan, Human islets of langerhans express Fas ligand and undergo apoptosis in response to interleukin-1beta and Fas ligation, Diabetes, 47 (1998) 727-732. 
80. M. Pericin, A. Althage, S. Freigang, H. Hengartner, E. Rolland, P. Dupraz, B. Thorens, P. Aebischer, R.M. Zinkernagel, Allogeneic beta-islet cells correct diabetes and resist immune rejection, Proc Natl Acad Sci U S A, 99 (2002) 82038206.

81. P.M. Campbell, P.A. Senior, A. Salam, K. Labranche, D.L. Bigam, N.M. Kneteman, S. Imes, A. Halpin, E.A. Ryan, A.M. Shapiro, High risk of sensitization after failed islet transplantation, Am J Transplant, 7 (2007) 23112317.

82. B.J. Olack, C.J. Swanson, K.S. Flavin, D. Phelan, D.C. Brennan, N.H. White, P.E. Lacy, D.W. Scharp, N. Poindexter, T. Mohanakumar, Sensitization to HLA antigens in islet recipients with failing transplants, Transplant Proc, 29 (1997) 2268-2269.

83. Y. Morel, A. Truneh, R.W. Sweet, D. Olive, R.T. Costello, The TNF superfamily members LIGHT and CD154 (CD40 ligand) costimulate induction of dendritic cell maturation and elicit specific CTL activity, J Immunol, 167 (2001) 24792486.

84. J. Emamaullee, C. Toso, S. Merani, A.M. Shapiro, Costimulatory blockade with belatacept in clinical and experimental transplantation: a review, Expert Opin Biol Ther, 9 (2009) 789-796.

85. H. Hui, N. Khoury, X. Zhao, L. Balkir, E. D'Amico, A. Bullotta, E.D. Nguyen, A. Gambotto, R. Perfetti, Adenovirus-mediated XIAP gene transfer reverses the negative effects of immunosuppressive drugs on insulin secretion and cell viability of isolated human islets, Diabetes, 54 (2005) 424-433.

86. C.A. Bonham, L. Peng, X. Liang, Z. Chen, L. Wang, L. Ma, H. Hackstein, P.D. Robbins, A.W. Thomson, J.J. Fung, S. Qian, L. Lu, Marked prolongation of cardiac allograft survival by dendritic cells genetically engineered with NF-kappa $\mathrm{B}$ oligodeoxyribonucleotide decoys and adenoviral vectors encoding CTLA4-Ig, J Immunol, 169 (2002) 3382-3391.

87. S. Yoshimura, J. Bondeson, B.M. Foxwell, F.M. Brennan, M. Feldmann, Effective antigen presentation by dendritic cells is NF-kappaB dependent: coordinate regulation of $\mathrm{MHC}$, co-stimulatory molecules and cytokines, Int Immunol, 13 (2001) 675-683.

88. M. Li, X. Zhang, X. Zheng, D. Lian, Z.X. Zhang, W. Ge, J. Yang, C. Vladau, M. Suzuki, D. Chen, R. Zhong, B. Garcia, A.M. Jevnikar, W.P. Min, Immune modulation and tolerance induction by RelB-silenced dendritic cells through RNA interference, J Immunol, 178 (2007) 5480-5487. 
89. M. Li, H. Qian, T.E. Ichim, W.W. Ge, I.A. Popov, K. Rycerz, J. Neu, D. White, R. Zhong, W.P. Min, Induction of RNA interference in dendritic cells, Immunol Res, 30 (2004) 215-230.

90. J.A. Hill, T.E. Ichim, K.P. Kusznieruk, M. Li, X. Huang, X. Yan, R. Zhong, E. Cairns, D.A. Bell, W.P. Min, Immune modulation by silencing IL-12 production in dendritic cells using small interfering RNA, J Immunol, 171 (2003) 691-696.

91. X. Liang, L. Lu, Z. Chen, T. Vickers, H. Zhang, J.J. Fung, S. Qian, Administration of dendritic cells transduced with antisense oligodeoxyribonucleotides targeting CD80 or CD86 prolongs allograft survival, Transplantation, 76 (2003) 721-729.

92. M. Suzuki, X. Zhang, X. Zheng, C. Vladau, M. Li, D. Chen, W. Min, Immune modulation through silencing CD80 and CD86 in dendritic cells using siRNA, J Immunol, 178 (2007) 84.

93. J. Xiang, X. Gu, S. Qian, Z. Chen, Graded function of CD80 and CD86 in initiation of T-cell immune response and cardiac allograft survival, Transpl Int, 21 (2008) 163-168.

94. M.H. Karimi, P. Ebadi, A.A. Pourfathollah, Z.S. Soheili, S. Samiee, Z. Ataee, S.Z. Tabei, S.M. Moazzeni, Immune modulation through RNA interferencemediated silencing of CD40 in dendritic cells, Cell Immunol, 259 (2009) 74-81.

95. X. Zheng, C. Vladau, X. Zhang, M. Suzuki, T.E. Ichim, Z.X. Zhang, M. Li, E. Carrier, B. Garcia, A.M. Jevnikar, W.P. Min, A novel in vivo siRNA delivery system specifically targeting dendritic cells and silencing CD40 genes for immunomodulation, Blood, 113 (2009) 2646-2654.

96. C. Zhu, H. Xu, G. Zhang, C. Lu, M. Ji, W. Wu, Myeloid differentiation factor 88silenced bone marrow-derived dendritic cells exhibit enhanced tolerogenicity in intestinal transplantation in rats, Transplant Proc, 40 (2008) 1625-1628.

97. C. Zhu, H. Xu, J. Zhang, K. Wang, P. Zhu, Donor bone-marrow suppressor of cytokine signaling-1-silenced dendritic cells prolong rat intestinal allograft survival, Transplant Proc, 40 (2008) 3707-3710.

98. L.H. Bouwman, Z. Ling, G. Duinkerken, D.G. Pipeleers, B.O. Roep, HLA incompatibility and immunogenicity of human pancreatic islet preparations cocultured with blood cells of healthy donors, Hum Immunol, 66 (2005) 494-500. 
99. K. Haga, N.A. Lemp, C.R. Logg, J. Nagashima, E. Faure-Kumar, G.G. Gomez, C.A. Kruse, R. Mendez, R. Stripecke, N. Kasahara, J.C. Cicciarelli, Permanent, lowered HLA class I expression using lentivirus vectors with shRNA constructs: averting cytotoxicity by alloreactive T lymphocytes, Transplant Proc, 38 (2006) 3184-3188.

100. K. Hacke, R. Falahati, L. Flebbe-Rehwaldt, N. Kasahara, K.M. Gaensler, Suppression of HLA expression by lentivirus-mediated gene transfer of siRNA cassettes and in vivo chemoselection to enhance hematopoietic stem cell transplantation, Immunol Res, 44 (2009) 112-126.

101. C. Figueiredo, A. Seltsam, U. Holtkamp, R. Blasczyk, Stable knock down of nonacceptable HLA mismatches in solid organ transplantation, Hum Immunol, 65 (2004) S7.

102. C. Figueiredo, A. Seltsam, R. Blasczyk, Class-, gene-, and group-specific HLA silencing by lentiviral shRNA delivery, J Mol Med, 84 (2006) 425-437.

103. S. Marzorati, A. Pileggi, C. Ricordi, Allogeneic islet transplantation, Expert Opin Biol Ther, 7 (2007) 1627-1645.

104. X. Zheng, X. Zhang, H. Sun, B. Feng, M. Li, G. Chen, C. Vladau, D. Chen, M. Suzuki, L. Min, W. Liu, R. Zhong, B. Garcia, A. Jevnikar, W.P. Min, Protection of renal ischemia injury using combination gene silencing of complement 3 and caspase 3 genes, Transplantation, 82 (2006) 1781-1786.

105. X. Zhang, X. Zheng, H. Sun, B. Feng, G. Chen, C. Vladau, M. Li, D. Chen, M. Suzuki,L. Min, W. Liu, B. Garcia, R. Zhong, W.P. Min, Prevention of renal ischemic injury by silencing the expression of renal caspase 3 and caspase 8 , Transplantation, 82 (2006) 1728-1732.

106. J.L. Contreras, M. Vilatoba, C. Eckstein, G. Bilbao, J. Anthony Thompson, D.E. Eckhoff, Caspase-8 and caspase-3 small interfering RNA decreases ischemia/reperfusion injury to the liver in mice, Surgery, 136 (2004) 390-400.

107. G. Cheng, L. Zhu, R.I. Mahato, Caspase-3 gene silencing for inhibiting apoptosis in insulinoma cells and human islets, Mol Pharm, 5 (2008) 1093-1102.

108. B.R. Burkhardt, R. Lyle, K. Qian, A.S. Arnold, H. Cheng, M.A. Atkinson, Y.C. Zhang, Efficient delivery of siRNA into cytokine-stimulated insulinoma cells silences Fas expression and inhibits Fas-mediated apoptosis, FEBS Lett, 580 (2006) 553-560.

109. J. Wang, W. Li, J. Min, Q. Ou, J. Chen, Fas siRNA reduces apoptotic cell death of allogeneic-transplanted hepatocytes in mouse spleen, Transplant Proc, 35 (2003) 1594-1595. 
110. X. Li, J.F. Zhang, M.Q. Lu, Y. Yang, C. Xu, H. Li, G.S. Wang, C.J. Cai, G.H. Chen, Alleviation of ischemia-reperfusion injury in rat liver transplantation by induction of small interference RNA targeting Fas, Langenbecks Arch Surg, 392 (2007) 345-351.

111. X. Zheng, D. Lian, A. Wong, M. Bygrave, T.E. Ichim, M. Khoshniat, X. Zhang, H. Sun, T. De Zordo, J.C. Lacefield, B. Garcia, A.M. Jevnikar, W.P. Min, Novel small interfering RNA-containing solution protecting donor organs in heart transplantation, Circulation, 120 (2009) 1099-1107.

112. X. Zheng, X. Zhang, B. Feng, H. Sun, M. Suzuki, T. Ichim, N. Kubo, A. Wong, L.R. Min, M.E. Budohn, B. Garcia, A.M. Jevnikar, W.P. Min, Gene silencing of complement C5a receptor using siRNA for preventing ischemia/reperfusion injury, Am J Pathol, 173 (2008) 973-980.

113. C. McCabe, T. O'Brien, Beta cell cytoprotection using lentiviral vector-based iNOS-specific shRNA delivery, Biochem Biophys Res Commun, 357 (2007) 7580.

114. C. McCabe, T. O'Brien, The rational design of beta cell cytoprotective gene transfer strategies: targeting deleterious iNOS expression, Mol Biotechnol, 37 (2007) 38-47.

115. F. Li, R.I. Mahato, iNOS gene silencing prevents inflammatory cytokine-induced beta-cell apoptosis, Mol Pharm, 5 (2008) 407-417.

116. D. De Paula, M.V. Bentley, R.I. Mahato, Effect of iNOS and NF-kappaB gene silencing on beta-cell survival and function, J Drug Target, 15 (2007) 358-369.

117. F. Li, R.I. Mahato, Bipartite vectors for co-expression of a growth factor cDNA and short hairpin RNA against an apoptotic gene, J Gene Med, 11 (2009) 764771.

118. O. Pinkenburg, J. Platz, C. Beisswenger, C. Vogelmeier, R. Bals, Inhibition of NF-kappaB mediated inflammation by siRNA expressed by recombinant adenoassociated virus, J Virol Methods, 120 (2004) 119-122.

119. B. Feng, G. Chen, X. Zheng, H. Sun, X. Zhang, Z.X. Zhang, Y. Xiang, T.E. Ichim, B. Garcia, P. Luke, A.M. Jevnikar, W.P. Min, Small interfering RNA targeting RelB protects against renal ischemia-reperfusion injury, Transplantation, 87 (2009) 1283-1289.

120. Y.S. Lee, K. Nakahara, J.W. Pham, K. Kim, Z. He, E.J. Sontheimer, R.W. Carthew, Distinct roles for drosophila Dicer-1 and Dicer-2 in the siRNA/miRNA silencing pathways, Cell, 117 (2004) 69-81. 
121. L. Wang, F.Y. Mu, A Web-based design center for vector-based siRNA and siRNA cassette, Bioinformatics, 20 (2004) 1818-1820.

122. Y. Naito, T. Yamada, K. Ui-Tei, S. Morishita, K. Saigo, siDirect: highly effective, target-specific siRNA design software for mammalian RNA interference, Nucleic Acids Res, 32 (2004) W124-129.

123. B. Yuan, R. Latek, M. Hossbach, T. Tuschl, F. Lewitter, siRNA selection server: an automated siRNA oligonucleotide prediction server, Nucleic Acids Res, 32 (2004) W130-134.

124. A. Henschel, F. Buchholz, B. Habermann, DEQOR: a web-based tool for the design and quality control of siRNAs, Nucleic Acids Res, 32 (2004) W113-120.

125. L. Li, X. Lin, A. Khvorova, S.W. Fesik, Y. Shen, Defining the optimal parameters for hairpin-based knockdown constructs, Rna, 13 (2007) 1765-1774.

126. J. Harborth, S.M. Elbashir, K. Vandenburgh, H. Manninga, S.A. Scaringe, K. Weber, T. Tuschl, Sequence, chemical, and structural variation of small interfering RNAs and short hairpin RNAs and the effect on mammalian gene silencing, Antisense Nucleic Acid Drug Dev, 13 (2003) 83-105.

127. A.L. Jackson, J. Burchard, J. Schelter, B.N. Chau, M. Cleary, L. Lim, P.S. Linsley, Widespread siRNA "off-target" transcript silencing mediated by seed region sequence complementarity, Rna, 12 (2006) 1179-1187.

128. A.D. Judge, V. Sood, J.R. Shaw, D. Fang, K. McClintock, I. MacLachlan, Sequence-dependent stimulation of the mammalian innate immune response by synthetic siRNA, Nat Biotechnol, 23 (2005) 457-462.

129. D.J. Taxman, L.R. Livingstone, J. Zhang, B.J. Conti, H.A. Iocca, K.L. Williams, J.D. Lich, J.P. Ting, W. Reed, Criteria for effective design, construction, and gene knockdown by shRNA vectors, BMC Biotechnol, 6 (2006) 7.

130. N. Hernandez, Small nuclear RNA genes: a model system to study fundamental mechanisms of transcription, J Biol Chem, 276 (2001) 26733-26736.

131. J. Song, S. Pang, Y. Lu, R. Chiu, Poly(U) and polyadenylation termination signals are interchangeable for terminating the expression of shRNA from a pol II promoter, Biochem Biophys Res Commun, 323 (2004) 573-578.

132. Y. Zeng, E.J. Wagner, B.R. Cullen, Both natural and designed micro RNAs can inhibit the expression of cognate mRNAs when expressed in human cells, Mol Cell, 9 (2002) 1327-1333. 
133. M.A. Denti, A. Rosa, O. Sthandier, F.G. De Angelis, I. Bozzoni, A new vector, based on the PolII promoter of the U1 snRNA gene, for the expression of siRNAs in mammalian cells, Mol Ther, 10 (2004) 191-199.

134. M. Huang, F.J. Jia, Y.C. Yan, L.H. Guo, Y.P. Li, Transactivated minimal E1b promoter is capable of driving the expression of short hairpin RNA, J Virol Methods, 134 (2006) 48-54.

135. X.G. Xia, H. Zhou, H. Ding, B. Affar el, Y. Shi, Z. Xu, An enhanced U6 promoter for synthesis of short hairpin RNA, Nucleic Acids Res, 31 (2003) e100.

136. S.T. Ong, F. Li, J. Du, Y.W. Tan, S. Wang, Hybrid cytomegalovirus enhancer-h1 promoter-based plasmid and baculovirus vectors mediate effective RNA interference, Hum Gene Ther, 16 (2005) 1404-1412.

137. Z. Hassani, J.C. Francois, G. Alfama, G.M. Dubois, M. Paris, C. Giovannangeli, B.A. Demeneix, A hybrid CMV-H1 construct improves efficiency of PEIdelivered shRNA in the mouse brain, Nucleic Acids Res, 35 (2007) e65.

138. M.S. Brenz Verca, P. Weber, C. Mayer, C. Graf, D. Refojo, R. Kuhn, I. Grummt, B. Lutz, Development of a species-specific RNA polymerase I-based shRNA expression vector, Nucleic Acids Res, 35 (2007) e10.

139. S.K. Lee, P. Kumar, Conditional RNAi: towards a silent gene therapy, Adv Drug Deliv Rev, 61 (2009) 650-664.

140. F. Czauderna, A. Santel, M. Hinz, M. Fechtner, B. Durieux, G. Fisch, F. Leenders, W. Arnold, K. Giese, A. Klippel, J. Kaufmann, Inducible shRNA expression for application in a prostate cancer mouse model, Nucleic Acids Res, $31(2003)$ e127.

141. M. van de Wetering, I. Oving, V. Muncan, M.T. Pon Fong, H. Brantjes, D. van Leenen, F.C. Holstege, T.R. Brummelkamp, R. Agami, H. Clevers, Specific inhibition of gene expression using a stably integrated, inducible smallinterfering-RNA vector, EMBO Rep, 4 (2003) 609-615.

142. M. Higuchi, R. Tsutsumi, H. Higashi, M. Hatakeyama, Conditional gene silencing utilizing the lac repressor reveals a role of SHP-2 in cagA-positive helicobacter pylori pathogenicity, Cancer Sci, 95 (2004) 442-447.

143. J.E. Heinonen, A.J. Mohamed, B.F. Nore, C.I. Smith, Inducible H1 promoterdriven lentiviral siRNA expression by stuffer reporter deletion, Oligonucleotides, 15 (2005) 139-144. 
144. H.A. Kim, B.W. Lee, D. Kang, J.H. Kim, S.H. Ihm, M. Lee, Delivery of hypoxiainducible VEGF gene to rat islets using polyethylenimine, J Drug Target, 17 (2009) 1-9.

145. B.W. Lee, H.Y. Chae, T.T. Tuyen, D. Kang, H.A. Kim, M. Lee, S.H. Ihm, A comparison of non-viral vectors for gene delivery to pancreatic beta-cells: delivering a hypoxia-inducible vascular endothelial growth factor gene to rat islets, Int J Mol Med, 23 (2009) 757-762.

146. R. Chai, S. Chen, J. Ding, P.A. Grayburn, Efficient, glucose responsive and isletspecific transgene expression by a modified rat insulin promoter, Gene Ther, 16 (2009) 1202-1209.

147. H. Iwakura, K. Hosoda, C. Son, J. Fujikura, T. Tomita, M. Noguchi, H. Ariyasu, K. Takaya, H. Masuzaki, Y. Ogawa, T. Hayashi, G. Inoue, T. Akamizu, H. Hosoda, M. Kojima, H. Itoh, S. Toyokuni, K. Kangawa, K. Nakao, Analysis of rat insulin II promoter-ghrelin transgenic mice and rat glucagon promoter-ghrelin transgenic mice, J Biol Chem, 280 (2005) 15247-15256.

148. M. Grzech, M. Dahlhoff, N. Herbach, F.A. Habermann, I. Renner-Muller, R. Wanke, H. Flaswinkel, E. Wolf, M.R. Schneider, Specific transgene expression in mouse pancreatic beta-cells under the control of the porcine insulin promoter, Mol Cell Endocrinol, 315 (2010) 219-224.

149. T. Avnit-Sagi, L. Kantorovich, S. Kredo-Russo, E. Hornstein, M.D. Walker, The promoter of the pri-miR-375 gene directs expression selectively to the endocrine pancreas, PLoS One, 4 (2009) e5033.

150. D. Siolas, C. Lerner, J. Burchard, W. Ge, P.S. Linsley, P.J. Paddison, G.J. Hannon, M.A. Cleary, Synthetic shRNAs as potent RNAi triggers, Nat Biotechnol, 23 (2005) 227-231.

151. L. Jeanson-Leh, J. Blondeau, A. Galy, Optimization of short hairpin RNA for lentiviral-mediated RNAi against WAS, Biochem Biophys Res Commun, 362 (2007) 498-503.

152. D. Boden, O. Pusch, R. Silbermann, F. Lee, L. Tucker, B. Ramratnam, Enhanced gene silencing of HIV-1 specific siRNA using microRNA designed hairpins, Nucleic Acids Res, 32 (2004) 1154-1158.

153. J.M. Silva, M.Z. Li, K. Chang, W. Ge, M.C. Golding, R.J. Rickles, D. Siolas, G. Hu, P.J. Paddison, M.R. Schlabach, N. Sheth, J. Bradshaw, J. Burchard, A. Kulkarni, G. Cavet, R. Sachidanandam, W.R. McCombie, M.A. Cleary, S.J. Elledge, G.J. Hannon, Second-generation shRNA libraries covering the mouse and human genomes, Nat Genet, 37 (2005) 1281-1288. 
154. F. Stegmeier, G. Hu, R.J. Rickles, G.J. Hannon, S.J. Elledge, A lentiviral microRNA-based system for single-copy polymerase II-regulated RNA interference in mammalian cells, Proc Natl Acad Sci U S A, 102 (2005) 1321213217.

155. R.L. Boudreau, A.M. Monteys, B.L. Davidson, Minimizing variables among hairpin-based RNAi vectors reveals the potency of shRNAs, RNA, 14 (2008) 1834-1844.

156. R.L. Boudreau, I. Martins, B.L. Davidson, Artificial microRNAs as siRNA shuttles: improved safety as compared to shRNAs in vitro and in vivo, Mol Ther, 17 (2009) 169-175.

157. T.T. Nielsen, I. Marion, L. Hasholt, C. Lundberg, Neuron-specific RNA interference using lentiviral vectors, J Gene Med, 11 (2009) 559-569.

158. K.H. Chung, C.C. Hart, S. Al-Bassam, A. Avery, J. Taylor, P.D. Patel, A.B. Vojtek, D.L. Turner, Polycistronic RNA polymerase II expression vectors for RNA interference based on BIC/miR-155, Nucleic Acids Res, 34 (2006) e53.

159. L.A. Aagaard, J. Zhang, K.J. von Eije, H. Li, P. Saetrom, M. Amarzguioui, J.J. Rossi, Engineering and optimization of the miR-106b cluster for ectopic expression of multiplexed anti-HIV RNAs, Gene Ther, 15 (2008) 1536-1549.

160. L.J. Scherer, R. Frank, J.J. Rossi, Optimization and characterization of tRNAshRNA expression constructs, Nucleic Acids Res, 35 (2007) 2620-2628.

161. H. Kawasaki, K. Taira, Short hairpin type of dsRNAs that are controlled by tRNA(Val) promoter significantly induce RNAi-mediated gene silencing in the cytoplasm of human cells, Nucleic Acids Res, 31 (2003) 700-707.

162. D. Boden, O. Pusch, F. Lee, L. Tucker, P.R. Shank, B. Ramratnam, Promoter choice affects the potency of HIV-1 specific RNA interference, Nucleic Acids Res, 31 (2003) 5033-5038.

163. T. Kuwabara, M. Warashina, M. Sano, H. Tang, F. Wong-Staal, E. Munekata, K. Taira, Recognition of engineered tRNAs with an extended 3' end by Exportin-t (Xpo-t) and transport of tRNA-attached ribozymes to the cytoplasm in somatic cells, Biomacromolecules, 2 (2001) 1229-1242.

164. L. Zheng, J. Liu, S. Batalov, D. Zhou, A. Orth, S. Ding, P.G. Schultz, An approach to genomewide screens of expressed small interfering RNAs in mammalian cells, Proc Natl Acad Sci U S A, 101 (2004) 135-140. 
165. A.L. Jackson, S.R. Bartz, J. Schelter, S.V. Kobayashi, J. Burchard, M. Mao, B. Li, G. Cavet, P.S. Linsley, Expression profiling reveals off-target gene regulation by RNAi, Nat Biotechnol, 21 (2003) 635-637.

166. A.L. Jackson, P.S. Linsley, Recognizing and avoiding siRNA off-target effects for target identification and therapeutic application, Nat Rev Drug Discov, 9 (2010) 57-67.

167. D.D. Rao, J.S. Vorhies, N. Senzer, J. Nemunaitis, siRNA vs. shRNA: similarities and differences, Adv Drug Deliv Rev, 61 (2009) 746-759.

168. A.L. Jackson, J. Burchard, D. Leake, A. Reynolds, J. Schelter, J. Guo, J.M. Johnson, L. Lim, J. Karpilow, K. Nichols, W. Marshall, A. Khvorova, P.S. Linsley, Position-specific chemical modification of siRNAs reduces "off-target" transcript silencing, RNA, 12 (2006) 1197-1205.

169. X. Lin, X. Ruan, M.G. Anderson, J.A. McDowell, P.E. Kroeger, S.W. Fesik, Y. Shen, siRNA-mediated off-target gene silencing triggered by a $7 \mathrm{nt}$ complementation, Nucleic Acids Res, 33 (2005) 4527-4535.

170. S. Qiu, C.M. Adema, T. Lane, A computational study of off-target effects of RNA interference, Nucleic Acids Res, 33 (2005) 1834-1847.

171. Q. Du, H. Thonberg, J. Wang, C. Wahlestedt, Z. Liang, A systematic analysis of the silencing effects of an active siRNA at all single-nucleotide mismatched target sites, Nucleic Acids Res, 33 (2005) 1671-1677.

172. J.B. Bramsen, M.B. Laursen, C.K. Damgaard, S.W. Lena, B.R. Babu, J. Wengel, J. Kjems, Improved silencing properties using small internally segmented interfering RNAs, Nucleic Acids Res, 35 (2007) 5886-5897.

173. K. Ui-Tei, Y. Naito, S. Zenno, K. Nishi, K. Yamato, F. Takahashi, A. Juni, K. Saigo, Functional dissection of siRNA sequence by systematic DNA substitution: modified siRNA with a DNA seed arm is a powerful tool for mammalian gene silencing with significantly reduced off-target effect, Nucleic Acids Res, 36 (2008) 2136-2151.

174. J.T. Marques, T. Devosse, D. Wang, M. Zamanian-Daryoush, P. Serbinowski, R. Hartmann, T. Fujita, M.A. Behlke, B.R. Williams, A structural basis for discriminating between self and nonself double-stranded RNAs in mammalian cells, Nat Biotechnol, 24 (2006) 559-565.

175. D.H. Kim, M.A. Behlke, S.D. Rose, M.S. Chang, S. Choi, J.J. Rossi, Synthetic dsRNA Dicer substrates enhance RNAi potency and efficacy, Nat Biotechnol, 23 (2005) 222-226. 
176. J.T. Marques, B.R. Williams, Activation of the mammalian immune system by siRNAs, Nat Biotechnol, 23 (2005) 1399-1405.

177. L.R. Saunders, G.N. Barber, The dsRNA binding protein family: critical roles, diverse cellular functions, FASEB J, 17 (2003) 961-983.

178. V. Hornung, M. Guenthner-Biller, C. Bourquin, A. Ablasser, M. Schlee, S. Uematsu, A. Noronha, M. Manoharan, S. Akira, A. de Fougerolles, S. Endres, G. Hartmann, Sequence-specific potent induction of IFN-alpha by short interfering RNA in plasmacytoid dendritic cells through TLR7, Nat Med, 11 (2005) 263-270.

179. C.A. Sledz, M. Holko, M.J. de Veer, R.H. Silverman, B.R. Williams, Activation of the interferon system by short-interfering RNAs, Nat Cell Biol, 5 (2003) 834839.

180. H. Hemmi, O. Takeuchi, T. Kawai, T. Kaisho, S. Sato, H. Sanjo, M. Matsumoto, K. Hoshino, H. Wagner, K. Takeda, S. Akira, A Toll-like receptor recognizes bacterial DNA, Nature, 408 (2000) 740-745.

181. H.K. de Wolf, N. Johansson, A.T. Thong, C.J. Snel, E. Mastrobattista, W.E. Hennink, G. Storm, Plasmid CpG depletion improves degree and duration of tumor gene expression after intravenous administration of polyplexes, Pharm Res, 25 (2008) 1654-1662.

182. D. Grimm, M.A. Kay, Therapeutic application of RNAi: is mRNA targeting finally ready for prime time?, J Clin Invest, 117 (2007) 3633-3641.

183. K. Cheng, N. Yang, R.I. Mahato, TGF-beta1 gene silencing for treating liver fibrosis, Mol Pharm, 6 (2009) 772-779.

184. Y. Chen, R.I. Mahato, siRNA pool targeting different sites of human hepatitis B surface antigen efficiently inhibits HBV infection, J Drug Target, 16 (2008) 140148.

185. D. Grimm, M.A. Kay, Combinatorial RNAi: a winning strategy for the race against evolving targets?, Mol Ther, 15 (2007) 878-888.

186. S. Gonzalez, D. Castanotto, H. Li, S. Olivares, M.C. Jensen, S.J. Forman, J.J. Rossi, L.J. Cooper, Amplification of RNAi--targeting HLA mRNAs, Mol Ther, 11 (2005) 811-818.

187. H. Zhou, X.G. Xia, Z. Xu, An RNA polymerase II construct synthesizes shorthairpin RNA with a quantitative indicator and mediates highly efficient RNAi, Nucleic Acids Res, 33 (2005) e62. 
188. X.G. Xia, H. Zhou, E. Samper, S. Melov, Z. Xu, Pol II-expressed shRNA knocks down Sod2 gene expression and causes phenotypes of the gene knockout in mice, PLoS Genet, 2 (2006) e10.

189. D. Sun, M. Melegari, S. Sridhar, C.E. Rogler, L. Zhu, Multi-miRNA hairpin method that improves gene knockdown efficiency and provides linked multi-gene knockdown, Biotechniques, 41 (2006) 59-63.

190. M.S. Weinberg, A. Ely, S. Barichievy, C. Crowther, S. Mufamadi, S. Carmona, P. Arbuthnot, Specific inhibition of HBV replication in vitro and in vivo with expressed long hairpin RNA, Mol Ther, 15 (2007) 534-541.

191. S. Saayman, S. Barichievy, A. Capovilla, K.V. Morris, P. Arbuthnot, M.S. Weinberg, The efficacy of generating three independent anti-HIV-1 siRNAs from a single U6 RNA Pol III-expressed long hairpin RNA, PLoS One, 3 (2008) e2602.

192. Y.P. Liu, J. Haasnoot, B. Berkhout, Design of extended short hairpin RNAs for HIV-1 inhibition, Nucleic Acids Res, 35 (2007) 5683-5693.

193. Y.P. Liu, K.J. von Eije, N.C. Schopman, J.T. Westerink, O. ter Brake, J. Haasnoot, B. Berkhout, Combinatorial RNAi against HIV-1 using extended short hairpin RNAs, Mol Ther, 17 (2009) 1712-1723.

194. J.Y. Yu, J. Taylor, S.L. DeRuiter, A.B. Vojtek, D.L. Turner, Simultaneous inhibition of GSK3alpha and GSK3beta using hairpin siRNA expression vectors, Mol Ther, 7 (2003) 228-236.

195. A. Jazag, F. Kanai, H. Ijichi, K. Tateishi, T. Ikenoue, Y. Tanaka, M. Ohta, J. Imamura, B. Guleng, Y. Asaoka, T. Kawabe, M. Miyagishi, K. Taira, M. Omata, Single small-interfering RNA expression vector for silencing multiple transforming growth factor-beta pathway components, Nucleic Acids Res, 33 (2005) e131.

196. K.J. Shin, E.A. Wall, J.R. Zavzavadjian, L.A. Santat, J. Liu, J.I. Hwang, R. Rebres, T. Roach, W. Seaman, M.I. Simon, I.D. Fraser, A single lentiviral vector platform for microRNA-based conditional RNA interference and coordinated transgene expression, Proc Natl Acad Sci U S A, 103 (2006) 13759-13764.

197. S. Samakoglu, L. Lisowski, T. Budak-Alpdogan, Y. Usachenko, S. Acuto, R. Di Marzo, A. Maggio, P. Zhu, J.F. Tisdale, I. Riviere, M. Sadelain, A genetic strategy to treat sickle cell anemia by coregulating globin transgene expression and RNA interference, Nat Biotechnol, 24 (2006) 89-94.

198. L. Qiu, H. Wang, X. Xia, H. Zhou, Z. Xu, A construct with fluorescent indicators for conditional expression of miRNA, BMC Biotechnol, 8 (2008) 77. 
199. R. Juliano, J. Bauman, H. Kang, X. Ming, Biological barriers to therapy with antisense and siRNA oligonucleotides, Mol Pharm, 6 (2009) 686-695.

200. D.L. Lewis, J.A. Wolff, Systemic siRNA delivery via hydrodynamic intravascular injection, Adv Drug Deliv Rev, 59 (2007) 115-123.

201. S.P. Bradley, T.F. Kowalik, C. Rastellini, M.A. da Costa, A.B. Bloomenthal, L. Cicalese, G.P. Basadonna, M.E. Uknis, Successful incorporation of shortinterfering RNA into islet cells by in situ perfusion, Transplant Proc, 37 (2005) 233-236.

202. B. Lefebvre, B. Vandewalle, J. Longue, E. Moerman, B. Lukowiak, V. Gmyr, K. Maedler, J. Kerr-conte, F. Pattou, Efficient gene delivery and silencing of mouse and human pancreatic islets, BMC Biotechnol, 10 (2010) 28.

203. M.A. Behlke, Chemical modification of siRNAs for in vivo use, Oligonucleotides, 18 (2008) 305-319.

204. D.R. Corey, Chemical modification: the key to clinical application of RNA interference?, J Clin Invest, 117 (2007) 3615-3622.

205. D.V. Morrissey, K. Blanchard, L. Shaw, K. Jensen, J.A. Lockridge, B. Dickinson, J.A. McSwiggen, C. Vargeese, K. Bowman, C.S. Shaffer, B.A. Polisky, S. Zinnen, Activity of stabilized short interfering RNA in a mouse model of hepatitis B virus replication, Hepatology, 41 (2005) 1349-1356.

206. D.V. Morrissey, J.A. Lockridge, L. Shaw, K. Blanchard, K. Jensen, W. Breen, K. Hartsough, L. Machemer, S. Radka, V. Jadhav, N. Vaish, S. Zinnen, C. Vargeese, K. Bowman, C.S. Shaffer, L.B. Jeffs, A. Judge, I. MacLachlan, B. Polisky, Potent and persistent in vivo anti-HBV activity of chemically modified siRNAs, Nat Biotechnol, 23 (2005) 1002-1007.

207. S. Choung, Y.J. Kim, S. Kim, H.O. Park, Y.C. Choi, Chemical modification of siRNAs to improve serum stability without loss of efficacy, Biochem Biophys Res Commun, 342 (2006) 919-927.

208. F. Czauderna, M. Fechtner, S. Dames, H. Aygun, A. Klippel, G.J. Pronk, K. Giese, J. Kaufmann, Structural variations and stabilising modifications of synthetic siRNAs in mammalian cells, Nucleic Acids Res, 31 (2003) 2705-2716.

209. A. Santel, M. Aleku, O. Keil, J. Endruschat, V. Esche, B. Durieux, K. Loffler, M. Fechtner, T. Rohl, G. Fisch, S. Dames, W. Arnold, K. Giese, A. Klippel, J. Kaufmann, RNA interference in the mouse vascular endothelium by systemic administration of siRNA-lipoplexes for cancer therapy, Gene Ther, 13 (2006) 1360-1370. 
210. J.J. Turner, S.W. Jones, S.A. Moschos, M.A. Lindsay, M.J. Gait, MALDI-TOF mass spectral analysis of siRNA degradation in serum confirms an RNAse A-like activity, Mol Biosyst, 3 (2007) 43-50.

211. A.D. Judge, G. Bola, A.C. Lee, I. MacLachlan, Design of noninflammatory synthetic siRNA mediating potent gene silencing in vivo, Mol Ther, 13 (2006) 494-505.

212. M. Robbins, A. Judge, L. Liang, K. McClintock, E. Yaworski, I. MacLachlan, 2'O-methyl-modified RNAs act as TLR7 antagonists, Mol Ther, 15 (2007) 16631669.

213. P.L. Felgner, T.R. Gadek, M. Holm, R. Roman, H.W. Chan, M. Wenz, J.P. Northrop, G.M. Ringold, M. Danielsen, Lipofection: a highly efficient, lipidmediated DNA-transfection procedure, Proc Natl Acad Sci U S A, 84 (1987) 7413-7417.

214. A.S. Narang, L. Thoma, D.D. Miller, R.I. Mahato, Cationic lipids with increased DNA binding affinity for nonviral gene transfer in dividing and nondividing cells, Bioconjug Chem, 16 (2005) 156-168.

215. Y. Takahashi, M. Nishikawa, Y. Takakura, Nonviral vector-mediated RNA interference: its gene silencing characteristics and important factors to achieve RNAi-based gene therapy, Adv Drug Deliv Rev, 61 (2009) 760-766.

216. M. Breunig, U. Lungwitz, R. Liebl, A. Goepferich, Breaking up the correlation between efficacy and toxicity for nonviral gene delivery, Proc Natl Acad Sci U S A, 104 (2007) 14454-14459.

217. Y. Lee, H. Mo, H. Koo, J.Y. Park, M.Y. Cho, G.W. Jin, J.S. Park, Visualization of the degradation of a disulfide polymer, linear poly(ethylenimine sulfide), for gene delivery, Bioconjug Chem, 18 (2007) 13-18.

218. J. Hoon Jeong, L.V. Christensen, J.W. Yockman, Z. Zhong, J.F. Engbersen, W. Jong Kim, J. Feijen, S. Wan Kim, Reducible poly(amido ethylenimine) directed to enhance RNA interference, Biomaterials, 28 (2007) 1912-1917.

219. M. Breunig, C. Hozsa, U. Lungwitz, K. Watanabe, I. Umeda, H. Kato, A. Goepferich, Mechanistic investigation of poly(ethylene imine)-based siRNA delivery: disulfide bonds boost intracellular release of the cargo, J Control Release, 130 (2008) 57-63.

220. M. Stevenson, V. Ramos-Perez, S. Singh, M. Soliman, J.A. Preece, S.S. Briggs, M.L. Read, L.W. Seymour, Delivery of siRNA mediated by histidine-containing reducible polycations, J Control Release, 130 (2008) 46-56. 
221. X.L. Wang, R. Jensen, Z.R. Lu, A novel environment-sensitive biodegradable polydisulfide with protonatable pendants for nucleic acid delivery, J Control Release, 120 (2007) 250-258.

222. M. Ou, X.L. Wang, R. Xu, C.W. Chang, D.A. Bull, S.W. Kim, Novel biodegradable poly(disulfide amine)s for gene delivery with high efficiency and low cytotoxicity, Bioconjug Chem, 19 (2008) 626-633.

223. J.J. Green, J. Shi, E. Chiu, E.S. Leshchiner, R. Langer, D.G. Anderson, Biodegradable polymeric vectors for gene delivery to human endothelial cells, Bioconjug Chem, 17 (2006) 1162-1169.

224. D. Jere, C.X. Xu, R. Arote, C.H. Yun, M.H. Cho, C.S. Cho, Poly(beta-amino ester) as a carrier for si/shRNA delivery in lung cancer cells, Biomaterials, 29 (2008) 2535-2547.

225. R.E. Vandenbroucke, B.G. De Geest, S. Bonne, M. Vinken, T. Van Haecke, H. Heimberg, E. Wagner, V. Rogiers, S.C. De Smedt, J. Demeester, N.N. Sanders, Prolonged gene silencing in hepatoma cells and primary hepatocytes after small interfering RNA delivery with biodegradable poly(beta-amino esters), J Gene Med, 10 (2008) 783-794.

226. S.J. Tseng, S.C. Tang, Development of poly(amino ester glycol urethane)/siRNA polyplexes for gene silencing, Bioconjug Chem, 18 (2007) 1383-1390.

227. Z. Zhao, J. Wang, H.Q. Mao, K.W. Leong, Polyphosphoesters in drug and gene delivery, Adv Drug Deliv Rev, 55 (2003) 483-499.

228. H.Q. Mao, K.W. Leong, Design of polyphosphoester-DNA nanoparticles for nonviral gene delivery, Adv Genet, 53 (2005) 275-306.

229. J. Wang, P.C. Zhang, H.Q. Mao, K.W. Leong, Enhanced gene expression in mouse muscle by sustained release of plasmid DNA using PPE-EA as a carrier, Gene Ther, 9 (2002) 1254-1261.

230. T.M. Sun, J.Z. Du, L.F. Yan, H.Q. Mao, J. Wang, Self-assembled biodegradable micellar nanoparticles of amphiphilic and cationic block copolymer for siRNA delivery, Biomaterials, 29 (2008) 4348-4355.

231. J. DeRouchey, C. Schmidt, G.F. Walker, C. Koch, C. Plank, E. Wagner, J.O. Radler, Monomolecular assembly of siRNA and poly(ethylene glycol)-peptide copolymers, Biomacromolecules, 9 (2008) 724-732.

232. M.S. Shim, Y.J. Kwon, Controlled delivery of plasmid DNA and siRNA to intracellular targets using ketalized polyethylenimine, Biomacromolecules, 9 (2008) 444-455. 
233. J. Soutschek, A. Akinc, B. Bramlage, K. Charisse, R. Constien, M. Donoghue, S. Elbashir, A. Geick, P. Hadwiger, J. Harborth, M. John, V. Kesavan, G. Lavine, R.K. Pandey, T. Racie, K.G. Rajeev, I. Rohl, I. Toudjarska, G. Wang, S. Wuschko, D. Bumcrot, V. Koteliansky, S. Limmer, M. Manoharan, H.P. Vornlocher, Therapeutic silencing of an endogenous gene by systemic administration of modified siRNAs, Nature, 432 (2004) 173-178.

234. C. Wolfrum, S. Shi, K.N. Jayaprakash, M. Jayaraman, G. Wang, R.K. Pandey, K.G. Rajeev, T. Nakayama, K. Charrise, E.M. Ndungo, T. Zimmermann, V. Koteliansky, M. Manoharan, M. Stoffel, Mechanisms and optimization of in vivo delivery of lipophilic siRNAs, Nat Biotechnol, 25 (2007) 1149-1157.

235. C. Lorenz, P. Hadwiger, M. John, H.P. Vornlocher, C. Unverzagt, Steroid and lipid conjugates of siRNAs to enhance cellular uptake and gene silencing in liver cells, Bioorg Med Chem Lett, 14 (2004) 4975-4977.

236. K. Nishina, T. Unno, Y. Uno, T. Kubodera, T. Kanouchi, H. Mizusawa, T. Yokota, Efficient in vivo delivery of siRNA to the liver by conjugation of alphatocopherol, Mol Ther, 16 (2008) 734-740.

237. Y.L. Chiu, A. Ali, C.Y. Chu, H. Cao, T.M. Rana, Visualizing a correlation between siRNA localization, cellular uptake, and RNAi in living cells, Chem Biol, 11 (2004) 1165-1175.

238. S.A. Moschos, S.W. Jones, M.M. Perry, A.E. Williams, J.S. Erjefalt, J.J. Turner, P.J. Barnes, B.S. Sproat, M.J. Gait, M.A. Lindsay, Lung delivery studies using siRNA conjugated to TAT(48-60) and penetratin reveal peptide induced reduction in gene expression and induction of innate immunity, Bioconjug Chem, 18 (2007) 1450-1459.

239. A. Muratovska, M.R. Eccles, Conjugate for efficient delivery of short interfering RNA (siRNA) into mammalian cells, FEBS Lett, 558 (2004) 63-68.

240. S.H. Lee, S.H. Kim, T.G. Park, Intracellular siRNA delivery system using polyelectrolyte complex micelles prepared from VEGF siRNA-PEG conjugate and cationic fusogenic peptide, Biochem Biophys Res Commun, 357 (2007) 511516.

241. S.H. Kim, J.H. Jeong, S.H. Lee, S.W. Kim, T.G. Park, PEG conjugated VEGF siRNA for anti-angiogenic gene therapy, J Control Release, 116 (2006) 123-129.

242. S.H. Kim, J.H. Jeong, S.H. Lee, S.W. Kim, T.G. Park, Local and systemic delivery of VEGF siRNA using polyelectrolyte complex micelles for effective treatment of cancer, J Control Release, 129 (2008) 107-116. 
243. M. Oishi, Y. Nagasaki, K. Itaka, N. Nishiyama, K. Kataoka, Lactosylated poly(ethylene glycol)-siRNA conjugate through acid-labile beta-thiopropionate linkage to construct $\mathrm{pH}$-sensitive polyion complex micelles achieving enhanced gene silencing in hepatoma cells, J Am Chem Soc, 127 (2005) 1624-1625.

244. M. Oishi, Y. Nagasaki, N. Nishiyama, K. Itaka, M. Takagi, A. Shimamoto, Y. Furuichi, K. Kataoka, Enhanced growth inhibition of hepatic multicellular tumor spheroids by lactosylated poly(ethylene glycol)-siRNA conjugate formulated in PEGylated polyplexes, ChemMedChem, 2 (2007) 1290-1297.

245. Z. Medarova, M. Kumar, S.W. Ng, J. Yang, N. Barteneva, N.V. Evgenov, V. Petkova, A. Moore, Multifunctional magnetic nanocarriers for image-tagged siRNA delivery to intact pancreatic islets, Transplantation, 86 (2008) 1170-1177.

246. A.S. Narang, O. Sabek, A.O. Gaber, R.I. Mahato, Co-expression of vascular endothelial growth factor and interleukin-1 receptor antagonist improves human islet survival and function, Pharm Res, 23 (2006) 1970-1982.

247. J.R. Bain, J.C. Schisler, K. Takeuchi, C.B. Newgard, T.C. Becker, An adenovirus vector for efficient RNA interference-mediated suppression of target genes in insulinoma cells and pancreatic islets of langerhans, Diabetes, 53 (2004) 21902194.

248. M. Iezzi, L. Eliasson, M. Fukuda, C.B. Wollheim, Adenovirus-mediated silencing of synaptotagmin 9 inhibits Ca2+-dependent insulin secretion in islets, FEBS Lett, 579 (2005) 5241-5246.

249. M.V. Jensen, J.W. Joseph, O. Ilkayeva, S. Burgess, D. Lu, S.M. Ronnebaum, M. Odegaard, T.C. Becker, A.D. Sherry, C.B. Newgard, Compensatory responses to pyruvate carboxylase suppression in islet beta-cells: preservation of glucosestimulated insulin secretion, J Biol Chem, 281 (2006) 22342-22351.

250. Z.L. Li, W.J. Xu, P.X. Tian, X.M. Ding, X.H. Tian, X.S. Feng, J. Hou, Recombinant adenovirus-mediated RNA silencing of tissue factor expression in human islet: an in vitro study, Nan Fang Yi Ke Da Xue Xue Bao, 27 (2007) 12991302.

251. L. Marzban, A. Tomas, T.C. Becker, L. Rosenberg, J. Oberholzer, P.E. Fraser, P.A. Halban, C.B. Verchere, Small interfering RNA-mediated suppression of proislet amyloid polypeptide expression inhibits islet amyloid formation and enhances survival of human islets in culture, Diabetes, 57 (2008) 3045-3055.

252. L. Naldini, Lentiviruses as gene transfer agents for delivery to non-dividing cells, Curr Opin Biotechnol, 9 (1998) 457-463. 
253. J.C. Burns, T. Friedmann, W. Driever, M. Burrascano, J.K. Yee, Vesicular stomatitis virus $\mathrm{G}$ glycoprotein pseudotyped retroviral vectors: concentration to very high titer and efficient gene transfer into mammalian and nonmammalian cells, Proc Natl Acad Sci U S A, 90 (1993) 8033-8037.

254. G.P. Kobinger, S. Deng, J.P. Louboutin, M. Vatamaniuk, F. Matschinsky, J.F. Markmann, S.E. Raper, J.M. Wilson, Transduction of human islets with pseudotyped lentiviral vectors, Hum Gene Ther, 15 (2004) 211-219.

255. E.S. Fenjves, M.S. Ochoa, S. Cechin, C. Gay-Rabinstein, I. Perez-Alvarez, H. Ichii, A. Mendez, C. Ricordi, M.A. Curran, Protection of human pancreatic islets using a lentiviral vector expressing two genes: cFLIP and GFP, Cell Transplant, 17 (2008) 793-802.

256. Y. Lu, H. Dang, B. Middleton, Z. Zhang, L. Washburn, M. Campbell-Thompson, M.A. Atkinson, S.S. Gambhir, J. Tian, D.L. Kaufman, Bioluminescent monitoring of islet graft survival after transplantation, Mol Ther, 9 (2004) 428-435.

257. I.K. Park, J. Lasiene, S.H. Chou, P.J. Horner, S.H. Pun, Neuron-specific delivery of nucleic acids mediated by Tet1-modified poly(ethylenimine), J Gene Med, 9 (2007) 691-702.

258. J.R. Fernandes, V.F. Duvivier-Kali, M. Keegan, J. Hollister-Lock, A. Omer, S. Su, S. Bonner-Weir, S. Feng, J.S. Lee, R.C. Mulligan, G.C. Weir, Transplantation of islets transduced with CTLA4-Ig and TGFbeta using adenovirus and lentivirus vectors, Transpl Immunol, 13 (2004) 191-200.

259. N. Giannoukakis, Z. Mi, A. Gambotto, A. Eramo, C. Ricordi, M. Trucco, P. Robbins, Infection of intact human islets by a lentiviral vector, Gene Ther, 6 (1999) 1545-1551.

260. W.S. Gallichan, T. Kafri, T. Krahl, I.M. Verma, N. Sarvetnick, Lentivirusmediated transduction of islet grafts with interleukin 4 results in sustained gene expression and protection from insulitis, Hum Gene Ther, 9 (1998) 2717-2726.

261. Z. He, F. Wang, M. Kumagai-Braesch, J. Permert, J. Holgersson, Long-term gene expression and metabolic control exerted by lentivirus-transduced pancreatic islets, Xenotransplantation, 13 (2006) 195-203.

262. H. Callewaert, C. Gysemans, A.K. Cardozo, M. Elsner, M. Tiedge, D.L. Eizirik, C. Mathieu, Cell loss during pseudoislet formation hampers profound improvements in islet lentiviral transduction efficacy for transplantation purposes, Cell Transplant, 16 (2007) 527-537. 
263. A.R. Barbu, B. Bodin, M. Welsh, L. Jansson, N. Welsh, A perfusion protocol for highly efficient transduction of intact pancreatic islets of Langerhans, Diabetologia, 49 (2006) 2388-2391.

264. S.S. Kim, C. Ye, P. Kumar, I. Chiu, S. Subramanya, H. Wu, P. Shankar, N. Manjunath, Targeted delivery of siRNA to macrophages for anti-inflammatory treatment, Mol Ther, 18 (2010) 993-1001.

265. C.A. Delaney, D.L. Eizirik, Intracellular targets for nitric oxide toxicity to pancreatic beta-cells, Braz J Med Biol Res, 29 (1996) 569-579.

266. C. Southern, D. Schulster, I.C. Green, Inhibition of insulin secretion by interleukin-1 beta and tumour necrosis factor-alpha via an L-arginine-dependent nitric oxide generating mechanism, FEBS Lett, 276 (1990) 42-44.

267. C. McCabe, A. Samali, T. O'Brien, Beta cell cytoprotective strategies: establishing the relative roles for iNOS and ROS, Biochem Biophys Res Commun, 342 (2006) 1240-1248.

268. N. Beeharry, J.A. Chambers, R.G. Faragher, K.E. Garnett, I.C. Green, Analysis of cytokine-induced NO-dependent apoptosis using RNA interference or inhibition by $1400 \mathrm{~W}$, Nitric Oxide, 10 (2004) 112-118.

269. G.J. Hannon, RNA interference, Nature, 418 (2002) 244-251.

270. S.P. Bradley, C. Rastellini, M.A. da Costa, T.F. Kowalik, A.B. Bloomenthal, M. Brown, L. Cicalese, G.P. Basadonna, M.E. Uknis, Gene silencing in the endocrine pancreas mediated by short-interfering RNA, Pancreas, 31 (2005) 373-379.

271. R. Hagerkvist, D. Mokhtari, J.W. Myers, A. Tengholm, N. Welsh, siRNA produced by recombinant dicer mediates efficient gene silencing in islet cells, Ann N Y Acad Sci, 1040 (2005) 114-122.

272. S. Kim, I. Millet, H.S. Kim, J.Y. Kim, M.S. Han, M.K. Lee, K.W. Kim, R.S. Sherwin, M. Karin, M.S. Lee, NF-kappa B prevents beta cell death and autoimmune diabetes in NOD mice, Proc Natl Acad Sci U S A, 104 (2007) 19131918.

273. R. Eldor, A. Yeffet, K. Baum, V. Doviner, D. Amar, Y. Ben-Neriah, G. Christofori, A. Peled, J.C. Carel, C. Boitard, T. Klein, P. Serup, D.L. Eizirik, D. Melloul, Conditional and specific NF-kappaB blockade protects pancreatic beta cells from diabetogenic agents, Proc Natl Acad Sci U S A, 103 (2006) 5072-5077.

274. R.H. Ritchie, X. Sun, J.L. Bilszta, L.M. Gulluyan, G.J. Dusting, Cardioprotective actions of an $\mathrm{N}$-terminal fragment of annexin-1 in rat myocardium in vitro, Eur $\mathrm{J}$ Pharmacol, 461 (2003) 171-179. 
275. S. Abdelli, J. Ansite, R. Roduit, T. Borsello, I. Matsumoto, T. Sawada, N. Allaman-Pillet, H. Henry, J.S. Beckmann, B.J. Hering, C. Bonny, Intracellular stress signaling pathways activated during human islet preparation and following acute cytokine exposure, Diabetes, 53 (2004) 2815-2823.

276. I. Budihardjo, H. Oliver, M. Lutter, X. Luo, X. Wang, Biochemical pathways of caspase activation during apoptosis, Annu Rev Cell Dev Biol, 15 (1999) 269-290.

277. N. Zhang, A. Richter, J. Suriawinata, S. Harbaran, J. Altomonte, L. Cong, H. Zhang, K. Song, M. Meseck, J. Bromberg, H. Dong, Elevated vascular endothelial growth factor production in islets improves islet graft vascularization, Diabetes, 53 (2004) 963-970.

278. J.L. Contreras, G. Bilbao, C.A. Smyth, X.L. Jiang, D.E. Eckhoff, S.M. Jenkins, F.T. Thomas, D.T. Curiel, J.M. Thomas, Cytoprotection of pancreatic islets before and soon after transplantation by gene transfer of the anti-apoptotic Bcl-2 gene, Transplantation, 71 (2001) 1015-1023.

279. J. Saldeen, Cytokines induce both necrosis and apoptosis via a common bcl-2inhibitable pathway in rat insulin-producing cells, Endocrinology, 141 (2000) 2003-2010.

280. A.L. Scarim, M. Arnush, L.A. Blair, J. Concepcion, M.R. Heitmeier, D. Scheuner, R.J. Kaufman, J. Ryerse, R.M. Buller, J.A. Corbett, Mechanisms of beta-cell death in response to double-stranded (ds) RNA and interferon-gamma: dsRNA-dependent protein kinase apoptosis and nitric oxide-dependent necrosis, Am J Pathol, 159 (2001) 273-283.

281. J. Storling, J. Binzer, A.K. Andersson, R.A. Zullig, M. Tonnesen, R. Lehmann, G.A. Spinas, S. Sandler, N. Billestrup, T. Mandrup-Poulsen, Nitric oxide contributes to cytokine-induced apoptosis in pancreatic beta cells via potentiation of JNK activity and inhibition of Akt, Diabetologia, 48 (2005) 2039-2050.

282. M. Biarnes, M. Montolio, V. Nacher, M. Raurell, J. Soler, E. Montanya, Beta-cell death and mass in syngeneically transplanted islets exposed to short- and longterm hyperglycemia, Diabetes, 51 (2002) 66-72.

283. T. Holen, M. Amarzguioui, M.T. Wiiger, E. Babaie, H. Prydz, Positional effects of short interfering RNAs targeting the human coagulation trigger tissue factor, Nucleic Acids Res, 30 (2002) 1757-1766.

284. E.A. Bohula, A.J. Salisbury, M. Sohail, M.P. Playford, J. Riedemann, E.M. Southern, V.M. Macaulay, The efficacy of small interfering RNAs targeted to the type 1 insulin-like growth factor receptor (IGF1R) is influenced by secondary structure in the IGF1R transcript, J Biol Chem, 278 (2003) 15991-15997. 
285. L. Li, W. El-Kholy, C.J. Rhodes, P.L. Brubaker, Glucagon-like peptide-1 protects beta cells from cytokine-induced apoptosis and necrosis: role of protein kinase B, Diabetologia, 48 (2005) 1339-1349.

286. P. Augstein, J. Bahr, G. Wachlin, P. Heinke, S. Berg, E. Salzsieder, L. Harrison, Cytokines activate caspase- 3 in insulinoma cells of diabetes-prone NOD mice directly and via upregulation of Fas, J Autoimmun, 23 (2004) 301-309.

287. N. Liadis, K. Murakami, M. Eweida, A.R. Elford, L. Sheu, H.Y. Gaisano, R. Hakem, P.S. Ohashi, M. Woo, Caspase-3-dependent beta-cell apoptosis in the initiation of autoimmune diabetes mellitus, Mol Cell Biol, 25 (2005) 3620-3629.

288. M. Nakano, I. Matsumoto, T. Sawada, J. Ansite, J. Oberbroeckling, H.J. Zhang, N. Kirchhof, J. Shearer, D.E. Sutherland, B.J. Hering, Caspase-3 inhibitor prevents apoptosis of human islets immediately after isolation and improves islet graft function, Pancreas, 29 (2004) 104-109.

289. M.A. Carmell, L. Zhang, D.S. Conklin, G.J. Hannon, T.A. Rosenquist, Germline transmission of RNAi in mice, Nat Struct Biol, 10 (2003) 91-92.

290. T.R. Brummelkamp, R. Bernards, R. Agami, A system for stable expression of short interfering RNAs in mammalian cells, Science, 296 (2002) 550-553.

291. Y. Dorsett, T. Tuschl, siRNAs: applications in functional genomics and potential as therapeutics, Nat Rev Drug Discov, 3 (2004) 318-329.

292. J.A. Goss, A.P. Schock, F.C. Brunicardi, S.E. Goodpastor, A.J. Garber, G. Soltes, M. Barth, T. Froud, R. Alejandro, C. Ricordi, Achievement of insulin independence in three consecutive type-1 diabetic patients via pancreatic islet transplantation using islets isolated at a remote islet isolation center, Transplantation, 74 (2002) 1761-1766.

293. T. Berney, C. Ricordi, Islet cell transplantation: the future?, Langenbecks Arch Surg, 385 (2000) 373-378.

294. X. Jia, K. Cheng, R.I. Mahato, Coexpression of vascular endothelial growth factor and interleukin-1 receptor antagonist for improved human islet survival and function, Mol Pharm, 4 (2007) 199-207.

295. P.S. Mountford, A.G. Smith, Internal ribosome entry sites and dicistronic RNAs in mammalian transgenesis, Trends Genet, 11 (1995) 179-184.

296. R.I. Mahato, M. Lee, S. Han, A. Maheshwari, S.W. Kim, Intratumoral delivery of p2CMVmIL-12 using water-soluble lipopolymers, Mol Ther, 4 (2001) 130-138. 
297. H. Mizuguchi, Z. Xu, A. Ishii-Watabe, E. Uchida, T. Hayakawa, IRES-dependent second gene expression is significantly lower than cap-dependent first gene expression in a bicistronic vector, Mol Ther, 1 (2000) 376-382.

298. X. Cai, C.H. Hagedorn, B.R. Cullen, Human microRNAs are processed from capped, polyadenylated transcripts that can also function as mRNAs, RNA, 10 (2004) 1957-1966.

299. X. Zhu, L.A. Santat, M.S. Chang, J. Liu, J.R. Zavzavadjian, E.A. Wall, C. Kivork, M.I. Simon, I.D. Fraser, A versatile approach to multiple gene RNA interference using microRNA-based short hairpin RNAs, BMC Mol Biol, 8 (2007) 98.

300. R.L. Boudreau, I. Martins, B.L. Davidson, Artificial microRNAs as siRNA shuttles: improved safety as compared to shRNAsin vitro and in vivo, Mol Ther, 17 (2008) 167-175.

301. A. Khvorova, A. Reynolds, S.D. Jayasena, Functional siRNAs and miRNAs exhibit strand bias, Cell, 115 (2003) 209-216.

302. A. Reynolds, D. Leake, Q. Boese, S. Scaringe, W.S. Marshall, A. Khvorova, Rational siRNA design for RNA interference, Nat Biotechnol, 22 (2004) 326-330.

303. K. Ui-Tei, Y. Naito, F. Takahashi, T. Haraguchi, H. Ohki-Hamazaki, A. Juni, R. Ueda, K. Saigo, Guidelines for the selection of highly effective siRNA sequences for mammalian and chick RNA interference, Nucleic Acids Res, 32 (2004) 936948.

304. G. Cheng, L. Zhu, R.I. Mahato, Caspase-3 gene silencing for inhibiting apoptosis in insulinoma cells and human islets, Mol Pharm, 5 (2008) 1093-1102.

305. C.Y. Wang, F. Li, Y. Yang, H.Y. Guo, C.X. Wu, S. Wang, Recombinant baculovirus containing the diphtheria toxin A gene for malignant glioma therapy, Cancer Res, 66 (2006) 5798-5806.

306. B.H. Liu, Y. Yang, J.F. Paton, F. Li, J. Boulaire, S. Kasparov, S. Wang, GAL4NF-kappaB fusion protein augments transgene expression from neuronal promoters in the rat brain, Mol Ther, 14 (2006) 872-882.

307. X. Wang, E. Olmsted-Davis, A. Davis, S. Liu, Z. Li, J. Yang, F.C. Brunicardi, Specific targeting of pancreatic islet cells in vivo by insulin-promoter-driven adenoviral conjugated reporter genes, World J Surg, 30 (2006) 1543-1552.

308. M.A. Jordan, L. Wilson, Microtubules as a target for anticancer drugs, Nat Rev Cancer, 4 (2004) 253-265. 
309. E.K. Rowinsky, The development and clinical utility of the taxane class of antimicrotubule chemotherapy agents, Annu Rev Med, 48 (1997) 353-374.

310. M.A. Jordan, K. Kamath, How do microtubule-targeted drugs work? An overview, Curr Cancer Drug Targets, 7 (2007) 730-742.

311. Q. Chu, M. Vincent, D. Logan, J.A. Mackay, W.K. Evans, Taxanes as first-line therapy for advanced non-small cell lung cancer: a systematic review and practice guideline, Lung Cancer, 50 (2005) 355-374.

312. A. Montero, F. Fossella, G. Hortobagyi, V. Valero, Docetaxel for treatment of solid tumours: a systematic review of clinical data, Lancet Oncol, 6 (2005) 229239.

313. G.D. Leonard, T. Fojo, S.E. Bates, The role of ABC transporters in clinical practice, Oncologist, 8 (2003) 411-424.

314. P. Seve, C. Dumontet, Is class III beta-tubulin a predictive factor in patients receiving tubulin-binding agents?, Lancet Oncol, 9 (2008) 168-175.

315. Z.S. Chen, E. Hopper-Borge, M.G. Belinsky, I. Shchaveleva, E. Kotova, G.D. Kruh, Characterization of the transport properties of human multidrug resistance protein 7 (MRP7, ABCC10), Mol Pharmacol, 63 (2003) 351-358.

316. A.L. Risinger, E.M. Jackson, L.A. Polin, G.L. Helms, D.A. LeBoeuf, P.A. Joe, E. Hopper-Borge, R.F. Luduena, G.D. Kruh, S.L. Mooberry, The taccalonolides: microtubule stabilizers that circumvent clinically relevant taxane resistance mechanisms, Cancer Res, 68 (2008) 8881-8888.

317. W. Gradishar, Management of advanced breast cancer with the epothilone B analog, ixabepilone, Drug Des Devel Ther, 3 (2009) 163-171.

318. Y. Lu, C.M. Li, Z. Wang, C.R. Ross, 2nd, J. Chen, J.T. Dalton, W. Li, D.D. Miller, Discovery of 4-substituted methoxybenzoyl-aryl-thiazole as novel anticancer agents: synthesis, biological evaluation, and structure-activity relationships, J Med Chem, 52 (2009) 1701-1711.

319. K.L. Hennenfent, R. Govindan, Novel formulations of taxanes: a review. Old wine in a new bottle?, Ann Oncol, 17 (2006) 735-749.

320. G. Gaucher, R.H. Marchessault, J.C. Leroux, Polyester-based micelles and nanoparticles for the parenteral delivery of taxanes, J Control Release, 143 (2010) 2-12. 
321. M. Danquah, F. Li, C.B. Duke, 3rd, D.D. Miller, R.I. Mahato, Micellar delivery of bicalutamide and embelin for treating prostate cancer, Pharm Res, 26 (2009) 2081-2092.

322. H. Maeda, G.Y. Bharate, J. Daruwalla, Polymeric drugs for efficient tumortargeted drug delivery based on EPR-effect, Eur J Pharm Biopharm, 71 (2009) 409-419.

323. H. Maeda, J. Wu, T. Sawa, Y. Matsumura, K. Hori, Tumor vascular permeability and the EPR effect in macromolecular therapeutics: a review, J Control Release, 65 (2000) 271-284.

324. T. Minko, P. Kopeckova, V. Pozharov, J. Kopecek, HPMA copolymer bound adriamycin overcomes MDR1 gene encoded resistance in a human ovarian carcinoma cell line, J Control Release, 54 (1998) 223-233.

325. T.K. Bronich, P.A. Keifer, L.S. Shlyakhtenko, A.V. Kabanov, Polymer micelle with cross-linked ionic core, J Am Chem Soc, 127 (2005) 8236-8237.

326. D.J. Booser, F.J. Esteva, E. Rivera, V. Valero, L. Esparza-Guerra, W. Priebe, G.N. Hortobagyi, Phase II study of liposomal annamycin in the treatment of doxorubicin-resistant breast cancer, Cancer Chemother Pharmacol, 50 (2002) 6-8.

327. X. Dong, C.A. Mattingly, M.T. Tseng, M.J. Cho, Y. Liu, V.R. Adams, R.J. Mumper, Doxorubicin and paclitaxel-loaded lipid-based nanoparticles overcome multidrug resistance by inhibiting P-glycoprotein and depleting ATP, Cancer Res, 69 (2009) 3918-3926.

328. J.T. Hunt, Discovery of ixabepilone, Mol Cancer Ther, 8 (2009) 275-281.

329. M. Takeda, A. Mizokami, K. Mamiya, Y.Q. Li, J. Zhang, E.T. Keller, M. Namiki, The establishment of two paclitaxel-resistant prostate cancer cell lines and the mechanisms of paclitaxel resistance with two cell lines, Prostate, 67 (2007) 955967.

330. Y. Liu, Y. Li, H. Wang, J. Yu, H. Lin, D. Xu, Y. Wang, A. Liang, X. Liang, X. Zhang, M. Fu, H. Qian, C. Lin, BH3-based fusion artificial peptide induces apoptosis and targets human colon cancer, Mol Ther, 17 (2009) 1509-1516.

331. K. Muckova, J.S. Duffield, K.D. Held, J.V. Bonventre, A.M. Sheridan, cPLA2interacting protein, PLIP, causes apoptosis and decreases G1 phase in mesangial cells, Am J Physiol Renal Physiol, 290 (2006) F70-79.

332. T.H. Wang, H.S. Wang, Y.K. Soong, Paclitaxel-induced cell death: where the cell cycle and apoptosis come together, Cancer, 88 (2000) 2619-2628. 
333. K.S. Lee, H.C. Chung, S.A. Im, Y.H. Park, C.S. Kim, S.B. Kim, S.Y. Rha, M.Y. Lee, J. Ro, Multicenter phase II trial of genexol-PM, a cremophor-free, polymeric micelle formulation of paclitaxel, in patients with metastatic breast cancer, Breast Cancer Res Treat, 108 (2008) 241-250.

334. P.J. Marsac, S.L. Shamblin, L.S. Taylor, Theoretical and practical approaches for prediction of drug-polymer miscibility and solubility, Pharm Res, 23 (2006) 24172426.

335. J.P. Latere Dwan'Isa, L. Rouxhet, V. Preat, M.E. Brewster, A. Arien, Prediction of drug solubility in amphiphilic di-block copolymer micelles: the role of polymer-drug compatibility, Pharmazie, 62 (2007) 499-504.

336. G. Gaucher, R.H. Marchessault, J.C. Leroux, Polyester-based micelles and nanoparticles for the parenteral delivery of taxanes, J Control Release, 143 (2010) 2-12.

337. A.N. Lukyanov, V.P. Torchilin, Micelles from lipid derivatives of water-soluble polymers as delivery systems for poorly soluble drugs, Adv Drug Deliv Rev, 56 (2004) 1273-1289.

338. V.P. Torchilin, Lipid-core micelles for targeted drug delivery, Curr Drug Deliv, 2 (2005) 319-327.

339. A.V. Kabanov, E.V. Batrakova, V.Y. Alakhov, Pluronic block copolymers as novel polymer therapeutics for drug and gene delivery, J Control Release, 82 (2002) 189-212.

340. A.V. Kabanov, E.V. Batrakova, V.Y. Alakhov, Pluronic block copolymers for overcoming drug resistance in cancer, Adv Drug Deliv Rev, 54 (2002) 759-779.

341. A.V. Kabanov, E.V. Batrakova, V.Y. Alakhov, An essential relationship between ATP depletion and chemosensitizing activity of pluronic block copolymers, $\mathrm{J}$ Control Release, 91 (2003) 75-83.

342. B. Jeong, Y.H. Bae, D.S. Lee, S.W. Kim, Biodegradable block copolymers as injectable drug-delivery systems, Nature, 388 (1997) 860-862.

343. S. Saha, A. Ghosh, P. Mahato, S. Mishra, S.K. Mishra, E. Suresh, S. Das, A. Das, Specific recognition and sensing of $\mathrm{CN}$ - in sodium cyanide solution, Org Lett, 12 (2010) 3406-3409.

344. M. Danquah, T. Fujiwara, R.I. Mahato, Self-assembling methoxypoly(ethylene glycol)-b-poly(carbonate-co-L-lactide) block copolymers for drug delivery, Biomaterials, 31 (2010) 2358-2370. 
345. A. Lavasanifar, J. Samuel, G.S. Kwon, Micelles of poly(ethylene oxide)-blockpoly(N-alkyl stearate L-aspartamide): synthetic analogues of lipoproteins for drug delivery, J Biomed Mater Res, 52 (2000) 831-835.

346. A. Lavasanifar, J. Samuel, G.S. Kwon, Micelles self-assembled from poly(ethylene oxide)-block-poly(N-hexyl stearate L-aspartamide) by a solvent evaporation method: effect on the solubilization and haemolytic activity of amphotericin B, J Control Release, 77 (2001) 155-160.

347. K. Yasugi, Y. Nagasaki, M. Kato, K. Kataoka, Preparation and characterization of polymer micelles from poly(ethylene glycol)-poly(D,L-lactide) block copolymers as potential drug carrier, J Control Release, 62 (1999) 89-100.

348. F. Li, Y. Lu, W. Li, D.D. Miller, R.I. Mahato, Synthesis, formulation and in vitro evaluation of a novel microtubule destabilizer, SMART-100, J Control Release, 143 (2010) 151-158.

349. L.S. Koester, G.G. Ortega, P. Mayorga, V.L. Bassani, Mathematical evaluation of in vitro release profiles of hydroxypropylmethylcellulose matrix tablets containing carbamazepine associated to beta-cyclodextrin, Eur J Pharm Biopharm, 58 (2004) 177-179.

350. T. Yamamoto, M. Yokoyama, P. Opanasopit, A. Hayama, K. Kawano, Y. Maitani, What are determining factors for stable drug incorporation into polymeric micelle carriers? Consideration on physical and chemical characters of the micelle inner core, J Control Release, 123 (2007) 11-18.

351. S.Y. Leung, J. Jackson, H. Miyake, H. Burt, M.E. Gleave, Polymeric micellar paclitaxel phosphorylates Bcl-2 and induces apoptotic regression of androgenindependent LNCaP prostate tumors, Prostate, 44 (2000) 156-163.

352. K. Greish, A. Nagamitsu, J. Fang, H. Maeda, Copoly(styrene-maleic acid)pirarubicin micelles: high tumor-targeting efficiency with little toxicity, Bioconjug Chem, 16 (2005) 230-236.

353. X. Shuai, T. Merdan, A.K. Schaper, F. Xi, T. Kissel, Core-cross-linked polymeric micelles as paclitaxel carriers, Bioconjug Chem, 15 (2004) 441-448.

354. Y. Xu, F. Meng, R. Cheng, Z. Zhong, Reduction-sensitive reversibly crosslinked biodegradable micelles for triggered release of doxorubicin, Macromol Biosci, 9 (2009) 1254-1261.

355. L. Tian, L. Yan, J. Wang, H. Tat, K. Uhrich, Core crosslinkable polymeric micelles from PEG-lipid amphiphiles as drug carriers, J Mater Chem, 14 (2004) 2317-2324. 
356. A. Mahmud, X. Xiong, A. Lavasanifar, Novel self-associating poly(ethylene oxide)-block-poly(epsilon-caprolactone) block copolymers with functional side groups on the polyester block for drug delivery, Macromolecules, 39 (2006) 9419-9428.

357. A. Mahmud, S. Patel, O. Molavi, P. Choi, J. Samuel, A. Lavasanifar, Selfassociating poly(ethylene oxide)-b-poly( $\alpha$-cholesteryl carboxylate- $\varepsilon$-caprolactone) block copolymer for the solubilization of STAT-3 inhibitor cucurbitacin I, Biomacromolecules, 10 (2009) 471-478.

358. W.Y. Seow, Y.Y. Yang, Functional polycarbonates and their self-assemblies as promising non-viral vectors, J Control Release, 139 (2009) 40-47.

359. A. Lavasanifar, J. Samuel, G.S. Kwon, The effect of fatty acid substitution on the in vitro release of amphotericin B from micelles composed of poly(ethylene oxide)-block-poly(N-hexyl stearate-L-aspartamide), J Control Release, 79 (2002) 165-172.

360. K. Zhu, R. Hendren, K. Jensen, C. Pitt, Synthesis, properties, and biodegradation of poly(1,3-trimethylene carbonate), Macromolecules, 24 (1991) 1736-1740.

361. Y.W. Tang, R.S. Labow, J.P. Santerre, Enzyme induced biodegradation of polycarbonate-polyurethanes: dose dependence effect of cholesterol esterase, Biomaterials, 24 (2003) 2003-2011.

362. J. Gao, Y. Guo, Z. Gu, X. Zhang, Micellization and controlled release properties of methoxy poly(ethylene glycol)- $b$-poly(D,L-lactide- co -trimethylene carbonate) Front Chem China, 4 (2009) 104-109.

363. C. Allen, D. Maysinger, A. Eisenberg, Nano-engineering block copolymer aggregates for drug delivery, Colloids Surf B Biointerfaces, 16 (1999) 3-27.

364. Y. Geng, P. Dalhaimer, S. Cai, R. Tsai, M. Tewari, T. Minko, D.E. Discher, Shape effects of filaments versus spherical particles in flow and drug delivery, Nat Nanotechnol, 2 (2007) 249-255.

365. S. Cai, K. Vijayan, D. Cheng, E.M. Lima, D.E. Discher, Micelles of different morphologies: advantages of worm-like filomicelles of PEO-PCL in paclitaxel delivery, Pharm Res, 24 (2007) 2099-2109.

366. D.A. Christian, S. Cai, O.B. Garbuzenko, T. Harada, A.L. Zajac, T. Minko, D.E. Discher, Flexible filaments for in vivo imaging and delivery: persistent circulation of filomicelles opens the dosage window for sustained tumor shrinkage, Mol Pharm, 6 (2009) 1343-1352. 
367. H. Cui, Z. Chen, S. Zhong, K.L. Wooley, D.J. Pochan, Block copolymer assembly via kinetic control, Science, 317 (2007) 647-650.

368. J. Zhu, R.C. Hayward, Spontaneous generation of amphiphilic block copolymer micelles with multiple morphologies through interfacial instabilities, J Am Chem Soc, 130 (2008) 7496-7502.

369. J. Baram, E. Shirman, N. Ben-Shitrit, A. Ustinov, H. Weissman, I. Pinkas, S.G. Wolf, B. Rybtchinski, Control over self-assembly through reversible charging of the aromatic building blocks in photofunctional supramolecular fibers, $\mathrm{J}$ Am Chem Soc, 130 (2008) 14966-14967.

370. Q. Chen, H. Zhao, T. Ming, J. Wang, C. Wu, Nanopore extrusion-induced transition from spherical to cylindrical block copolymer micelles, J Am Chem Soc, 131 (2009) 16650-16651.

371. S. Jain, F.S. Bates, On the origins of morphological complexity in block copolymer surfactants, Science, 300 (2003) 460-464.

372. E.K. Park, S.B. Lee, Y.M. Lee, Preparation and characterization of methoxy poly(ethylene glycol)/poly(epsilon-caprolactone) amphiphilic block copolymeric nanospheres for tumor-specific folate-mediated targeting of anticancer drugs, Biomaterials, 26 (2005) 1053-1061.

373. G. Theyer, M. Schirmbock, T. Thalhammer, E.R. Sherwood, G. Baumgartner, G. Hamilton, Role of the MDR-1-encoded multiple drug resistance phenotype in prostate cancer cell lines, J Urol, 150 (1993) 1544-1547.

374. J.P. van Brussel, G.H. Mickisch, Multidrug resistance in prostate cancer, Onkologie, 26 (2003) 175-181.

375. C. Sanchez, P. Mendoza, H.R. Contreras, J. Vergara, J.A. McCubrey, C. Huidobro, E.A. Castellon, Expression of multidrug resistance proteins in prostate cancer is related with cell sensitivity to chemotherapeutic drugs, Prostate, 69 (2009) 1448-1459.

376. G. Szakacs, J.K. Paterson, J.A. Ludwig, C. Booth-Genthe, M.M. Gottesman, Targeting multidrug resistance in cancer, Nat Rev Drug Discov, 5 (2006) 219234.

377. A. Rahman, S.R. Husain, J. Siddiqui, M. Verma, M. Agresti, M. Center, A.R. Safa, R.I. Glazer, Liposome-mediated modulation of multidrug resistance in human HL-60 leukemia cells, J Natl Cancer Inst, 84 (1992) 1909-1915. 
378. A.M. Carcaboso, M.A. Elmeliegy, J. Shen, S.J. Juel, Z.M. Zhang, C. Calabrese, L. Tracey, C.M. Waters, C.F. Stewart, Tyrosine kinase inhibitor gefitinib enhances topotecan penetration of gliomas, Cancer Res, 70 (2010) 4499-4508.

379. P.J. Medina, S. Goodin, Lapatinib: a dual inhibitor of human epidermal growth factor receptor tyrosine kinases, Clin Ther, 30 (2008) 1426-1447.

380. C.L. Dai, A.K. Tiwari, C.P. Wu, X.D. Su, S.R. Wang, D.G. Liu, C.R. Ashby, Jr., Y. Huang, R.W. Robey, Y.J. Liang, L.M. Chen, C.J. Shi, S.V. Ambudkar, Z.S. Chen, L.W. Fu, Lapatinib (Tykerb, GW572016) reverses multidrug resistance in cancer cells by inhibiting the activity of ATP-binding cassette subfamily B member 1 and G member 2, Cancer Res, 68 (2008) 7905-7914.

381. Y.H. Kuang, T. Shen, X. Chen, K. Sodani, E. Hopper-Borge, A.K. Tiwari, J.W. Lee, L.W. Fu, Z.S. Chen, Lapatinib and erlotinib are potent reversal agents for MRP7 (ABCC10)-mediated multidrug resistance, Biochem Pharmacol, 79 (2010) 154-161.

382. D.M. Collins, J. Crown, N. O'Donovan, A. Devery, F. O'Sullivan, L. O'Driscoll, M. Clynes, R. O'Connor, Tyrosine kinase inhibitors potentiate the cytotoxicity of MDR-substrate anticancer agents independent of growth factor receptor status in lung cancer cell lines, Invest New Drugs, 28 (2010) 433-444.

383. X. Li, M.T. Lewis, J. Huang, C. Gutierrez, C.K. Osborne, M.F. Wu, S.G. Hilsenbeck, A. Pavlick, X. Zhang, G.C. Chamness, H. Wong, J. Rosen, J.C. Chang, Intrinsic resistance of tumorigenic breast cancer cells to chemotherapy, J Natl Cancer Inst, 100 (2008) 672-679.

384. F. Li, M. Danquah, R.I. Mahato, Synthesis and characterization of amphiphilic lipopolymers for micellar drug delivery, Biomacromolecules, 11 (2010) 26102620. 


\section{VITA}

Feng Li was born in Hangzhou, Zhejiang, China in 1979. He received his Bachelor of Pharmacy degree from Zhejiang University (ZJU), China, in 2002. He also received his Master of Science degree from National University of Singapore (NUS), Singapore, in 2006. He joined the graduate program in Pharmaceutical Sciences at the University of Tennessee Health Science Center (UTHSC), Memphis, in 2006 with Dr. Ram I. Mahato as major advisor. 